\title{
Dual pair correspondence in physics: oscillator realizations and representations
}

\author{
Thomas Basile, ${ }^{a}$ Euihun Joung, ${ }^{a}$ Karapet Mkrtchyan ${ }^{b}$ and Matin Mojaza ${ }^{c}$ \\ ${ }^{a}$ Department of Physics, Kyung Hee University, \\ Seoul 02447, Korea \\ ${ }^{b}$ Scuola Normale Superiore and INFN, Piazza dei Cavalieri 7, \\ 56126 Pisa, Italy \\ ${ }^{c}$ Albert-Einstein-Institut, Max-Planck-Institut für Gravitationsphysik, \\ 14476 Potsdam, Germany \\ E-mail: thomas.basile@khu.ac.kr, euihun.joung@khu.ac.kr, \\ karapet.mkrtchyan@sns.it, matin.mojaza@aei.mpg.de
}

ABstract: We study general aspects of the reductive dual pair correspondence, also known as Howe duality. We make an explicit and systematic treatment, where we first derive the oscillator realizations of all irreducible dual pairs: $\quad(G L(M, \mathbb{R}), G L(N, \mathbb{R})), \quad(G L(M, \mathbb{C}), G L(N, \mathbb{C})), \quad\left(U^{*}(2 M), U^{*}(2 N)\right)$, $\left(U\left(M_{+}, M_{-}\right), U\left(N_{+}, N_{-}\right)\right), \quad\left(O\left(N_{+}, N_{-}\right), S p(2 M, \mathbb{R})\right), \quad(O(N, \mathbb{C}), S p(2 M, \mathbb{C})) \quad$ and $\left(O^{*}(2 N), S p\left(M_{+}, M_{-}\right)\right)$. Then, we decompose the Fock space into irreducible representations of each group in the dual pairs for the cases where one member of the pair is compact as well as the first non-trivial cases of where it is non-compact. We discuss the relevance of these representations in several physical applications throughout this analysis. In particular, we discuss peculiarities of their branching properties. Finally, closed-form expressions relating all Casimir operators of two groups in a pair are established.

Keywords: Conformal and W Symmetry, Higher Spin Symmetry, Space-Time Symmetries

ARXIV EPRINT: 2006.07102 


\section{Contents}

1 Introduction $\quad 1$

1.1 Brief summary of the paper 4

1.2 Conventions 5

2 Generalities of the reductive dual pair correspondence $\quad 7$

2.1 Metaplectic representation 8

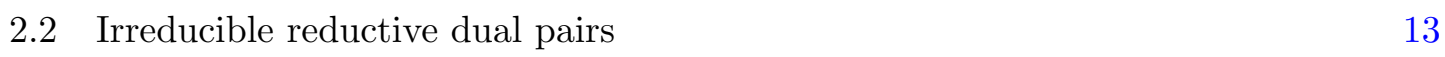

$\begin{array}{lll}2.3 & \text { Seesaw pairs } & 13\end{array}$

3 Dual pairs of type $\left(G L_{M}, G L_{N}\right) \quad 16$

$3.1(G L(M, \mathbb{R}), G L(N, \mathbb{R})) \subset S p(2 M N, \mathbb{R}) \quad 17$

$3.2(G L(M, \mathbb{C}), G L(N, \mathbb{C})) \subset S p(4 M N, \mathbb{R}) \quad 18$

$3.3\left(U^{*}(2 M), U^{*}(2 N)\right) \subset S p(8 M N, \mathbb{R}) \quad 19$

$3.4\left(U\left(M_{+}, M_{-}\right), U\left(N_{+}, N_{-}\right)\right) \subset S p\left(2\left(M_{+}+M_{-}\right)\left(N_{+}+N_{-}\right), \mathbb{R}\right) \quad 20$

4 Dual pairs of type $\left(O_{N}, S p_{2 M}\right) \quad \mathbf{2 1}$

$4.1\left(O\left(N_{+}, N_{-}\right), S p(2 M, \mathbb{R})\right) \subset S p\left(2 M\left(N_{+}+N_{-}\right), \mathbb{R}\right)$

$4.2(O(N, \mathbb{C}), S p(2 M, \mathbb{C})) \subset S p(4 N M, \mathbb{R}) \quad 23$

$4.3\left(O^{*}(2 N), S p\left(M_{+}, M_{-}\right)\right) \subset S p\left(4 N\left(M_{+}+M_{-}\right), \mathbb{R}\right)$

5 Representations of compact dual pairs $\quad \mathbf{2 5}$

$\begin{array}{lll}5.1(U(M), U(N)) & 27\end{array}$

$5.2\left(U\left(M_{+}, M_{-}\right), U(N)\right) \quad 29$

$5.3(O(N), S p(2 M, \mathbb{R})) \quad 32$

$5.4\left(O^{*}(2 N), S p(M)\right) \quad 35$

6 Representation of exceptionally compact dual pairs $\quad 37$

$6.1\left(O^{*}(2), S p\left(M_{+}, M_{-}\right)\right) \quad 37$

$\begin{array}{lll}6.2 & (S p(2 N, \mathbb{C}), O(1, \mathbb{C})) & 39\end{array}$

7 Representations of the simplest non-compact dual pairs $\quad 40$

$7.1(G L(N, \mathbb{R}), G L(1, \mathbb{R}))$ and $(G L(N, \mathbb{R}), G L(M, \mathbb{R})) \quad 40$

$7.2(G L(N, \mathbb{C}), G L(1, \mathbb{C}))$ and $(G L(N, \mathbb{C}), G L(M, \mathbb{C}))$

$7.3\left(U^{*}(2 N), U^{*}(2)\right)$ and $\left(U^{*}(2 N), U^{*}(2 M)\right) \quad 49$

$7.4(S p(2 N, \mathbb{R}), O(1,1))$ and $(S p(2 N, \mathbb{R}), O(M, M))$

$7.5(S p(2 N, \mathbb{C}), O(2, \mathbb{C}))$ and $(S p(2 N, \mathbb{C}), O(2 M, \mathbb{C}))$

8 Branching properties $\quad 61$

$\begin{array}{ll}\text { 8.1 Restriction to maximal compact subgroup } & 61\end{array}$

$\begin{array}{lll}8.2 & \text { Irreducibility under restriction } & 62\end{array}$ 
9.1 Duality $\left(G L_{M}, G L_{N}\right) \quad 65$

9.2 Duality $\left(O_{N}, S p_{2 M}\right) \quad 68$

$\begin{array}{ll}\text { A Representations of compact groups } & 71\end{array}$

A.1 Finite-dimensional representations of $G L_{N} \quad 72$

$\begin{array}{lll}\text { A.2 } & \text { Finite-dimensional representations of } O(N) & 76\end{array}$

$\begin{array}{lll}\text { A.3 Finite-dimensional representations of } S p(N) & 79\end{array}$

B Real forms of classical Lie groups and Lie algebras $\quad \mathbf{8 0}$

$\begin{array}{ll}\text { B.1 General linear Lie groups and Lie algebras } & 81\end{array}$

$\begin{array}{lll}\text { B.2 } & \text { Orthogonal Lie groups and Lie algebras } & 82\end{array}$

B.3 Symplectic Lie groups and Lie algebras $\quad 84$

$\begin{array}{ll}\text { C Seesaw pairs diagrams } & 85\end{array}$

$\begin{array}{lr}\text { D Derivation of Casimir relations } & \mathbf{8 7}\end{array}$

D.1 Relating different choices of Casimir operators for the same group $G L_{N} \quad 87$

$\begin{array}{lll}\text { D.2 Details on the Casimir relations for }\left(O_{N}, S p_{2 M}\right) & 89\end{array}$

\section{Introduction}

The reductive dual pair correspondence, also known as Howe duality [1,2], provides a useful mathematical framework to study a physical system, allowing for a straightforward analysis of its symmetries and spectrum. It finds applications in a wide range of subjects from low energy physics - such as in condensed matter physics, quantum optics and quantum information theory — to high energy physics — such as in twistor theory, supergravity, conformal field theories, scattering amplitudes and higher spin field theories. Despite its importance, the dual pair correspondence, as we will simply refer to it, is not among the most familiar mathematical concepts in the theoretical physics community, and particularly not in those of field and string theory, where it nevertheless appears quite often, either implicitly, or as an outcome of the analyses. For this reason, many times when the dual pair correspondence makes its occurrence in the physics literature, its role often goes unnoticed or is not being emphasized, albeit it is actually acting as an underlying governing principle.

In a nutshell, the dual pair correspondence is about the oscillator realization of Lie algebras. In quantum physics, oscillators realizations - that is, the description of physical systems in terms of creation and annihilation operators, $a$ and $a^{\dagger}$ - are ubiquitous, and their use in algebraically solving the quantum harmonic oscillator is a central and standard part of any undergraduate physics curriculum. Oscillator realizations are more generally encountered in many contemporary physical problems. It can therefore be valuable to study their properties in a more general and broader mathematical framework. The dual pair 
correspondence provides exactly such a framework, applicable to many unrelated physical problems.

The first oscillator realization of a Lie algebra was given by Jordan in 1935 [3] to describe the relation between the symmetric and the linear groups. This so-called JordanSchwinger map was used much later by Schwinger to realize the $\mathfrak{s u}(2)$ obeying angular momentum operators ${ }^{1}$ in terms of two pairs of ladder operators, $a, a^{\dagger}$ and $b, b^{\dagger}$, as follows

$$
J_{3}=\frac{1}{2}\left(a^{\dagger} a-b^{\dagger} b\right), \quad J_{+}=a^{\dagger} b, \quad J_{-}=b^{\dagger} a,
$$

thereby finding a gateway to construct all irreducible representation of $\mathfrak{s u}(2)$ in terms of two uncoupled quantum harmonic oscillators. The spin- $j$ representations then simply become the states with excitation number $2 j$ :

$$
N=a^{\dagger} a+b^{\dagger} b, \quad N\left|\Psi_{j}\right\rangle=2 j\left|\Psi_{j}\right\rangle .
$$

When $\mathfrak{s u}(2)$ is realized as above, there emerges another Lie algebra, $\mathfrak{u}(1)$ - generated by the number operator $N$ - which is not a subalgebra of the $\mathfrak{s u}(2)$. Uplifting this to Lie groups, we find that the spin- $j$ representations of $S U(2)$ are in one-to-one correspondence with the $U(1)$ representations; i.e. those labelled by the oscillator number $2 j$. This interplay between the two groups $S U(2)$ and $U(1)$ is the first example of the dual pair correspondence. A generalization of this mechanism to the duality between $U(4)$ and $U(N)$ was made by Wigner [4] to model a system in nuclear physics. Replacing the bosonic oscillators by fermionic ones, Racah extended the duality to the one between $S p(M)$ and $S p(N)$ in [5]. Subsequently, the method was widely used in various models of nuclear physics, such as the nuclear shell model and the interacting boson model (see e.g. [6, 7]).

Concerning non-compact Lie groups, an oscillator-like representation, named expansor, was introduced for the Lorentz group by Dirac in 1944 [8], and its fermionic counterpart, the expinor, was constructed by Harish-Chandra [9] (see also [10] and an historical note [11]). ${ }^{2}$ In 1963, Dirac also introduced an oscillator representation for the $3 \mathrm{~d}$ conformal group, which he referred to as a "remarkable representation" [15]. The analogous representation for the $4 \mathrm{~d}$ conformal group was constructed in [16] (see also [17, 18]). The oscillator realization and the dual pair correspondence are also closely related to twistor theory [19-21], where the dual group of the $4 \mathrm{~d}$ conformal group was interpreted as an internal symmetry group in the 'naive twistor particle theory' (see e.g. the first chapter of [22]).

Around the same time, oscillator representations were studied in mathematics by Segal [23], Shale [24] and by Weil [25] (hence, often referred to as Weil representations). Based on these earlier works (and others that we omit to mention), Howe eventually came up with the reductive dual pair correspondence in 1976 (published much later in [1,2]). In

\footnotetext{
${ }^{1}$ In this section, we mention several important works which went before the dual pair correspondence. Our intention, however, is not to select the most important contributions, but rather to illustrate the historical development of oscillator realizations and the dual pair correspondence in physics.

${ }^{2}$ These representations are closely related to Majorana's equation for infinite component spinor carrying a unitary representation of the Lorentz group, published in 1932 [12]. The complete classification of unitary and irreducible representations of the four-dimensional Lorentz group was later obtained in [9, 13, 14].
} 
short, he showed that there exists a one-to-one correspondence between oscillator representations of two mutually commuting subgroups of the symplectic group. Since then, many mathematicians contributed to the development of the subject (see e.g. reviews [26-28] and references therein). The dual pair correspondence is also referred to as the (local) theta correspondence and it has an intimate connection to the theory of automorphic forms (see, e.g., $[29,30])$.

In physics, oscillator realizations were studied also in the context of coherent states [3133]. In the 80-90's, Günaydin and collaborators extensively used oscillators to realize various super Lie algebras arising in supergravity theories; i.e. those of $S U(2,2 \mid N)$ [34, 35], $O S p(N \mid 4, \mathbb{R})[36,37]$ and $O S p\left(8^{*} \mid N\right)[38,39]$ (see also [40-42]). Part of the analysis of representations contained in this paper can be found already in these early references. However, in those papers, the (role of the) dual group was not (explicitly) considered, ${ }^{3}$ even though it implicitly appears in the tensor product decompositions. (Its relevance was, however, alluded to in [42].)

Higher spin field theory is another area where the oscillator realizations and the dual pair correspondence were fruitfully employed. Spinor oscillators were used to construct $4 \mathrm{~d}$ higher spin algebra by Fradkin and Vasiliev [43] and to identify the underlying representations by Konstein and Vasiliev [44]. Fradkin and Linetsky found that the extension of the work to $4 \mathrm{~d}$ conformal higher spin theory, or equivalently $5 \mathrm{~d}$ higher spin theory, requires a dual $\mathfrak{u}(1)$ algebra $[45,46]$. The same mechanism was used by Sezgin and Sundell in extending Vasiliev's 4d theory [47-49] to $5 \mathrm{~d}$ [50] and 7d [51], with the dual algebras $\mathfrak{u}(1)$ and $\mathfrak{s u}(2)$ (as we shall later see, the latter algebra is more appropriately interpreted as $\mathfrak{s p}(1)$ ). In 2003, Vasiliev generalized his theory to any dimensions using vector oscillators [52], and revisited its representation theory in [53]. In both of these works, the dual pair correspondence played a crucial role, and since then, it has been used several times within the context of higher spin theories. ${ }^{4}$

Despite the abundance of relevant works in physics, and reviews in mathematics, it is hard to find accessible references on the dual pair correspondence for physicists, with a notable exception of [70]. ${ }^{5}$ In physics heuristic approaches rather than systematic ones are common, whereas in the mathematics literature, the treatment is formal and explicit examples are rare. The current work is an attempt to close the gap between the physics and math literature. Specifically, we provide a systematic derivation of the oscillator realizations for essentially all dual pairs, as well as, in the relatively simple cases, an explicit decomposition of the corresponding Fock spaces into irreducible representations of each group of the pairs. To be self-contained, we included many basic elements of representation theory related to oscillators. Thus, there will be a considerable overlap with many earlier works mentioned previously. Nevertheless, we believe that our treatment could help

\footnotetext{
${ }^{3}$ Note that the isometry and R-symmetry groups follow a similar pairing pattern, but they are not reductive dual pairs.

${ }^{4}$ In fact, almost all of the higher spin literature is related to the dual pair correspondence in one way or another. For this reason, we mention just a few papers in which the duality is directly and explicitly used [53-69].

${ }^{5}$ The historical accounts given here are indebted to this review.
} 
in filling several gaps in the current understanding of the use and role of the dual pair correspondence in physics. As the subject of the dual pair correspondence is rich, we will not cover all that we intend to in a single paper. We instead complete our program in (at least) two follow-up papers; one of the same kind as this, but on more advanced issues, and another dedicated to physical applications. Although physical applications will be visited in depth in a sequel, we include brief comments on them throughout the current paper, as summarized below.

\subsection{Brief summary of the paper}

In section 2, we review generalities of the dual pair correspondence, starting with the precise statement of the duality. In section 2.1, we recall the definition of the metaplectic representation. In section 2.2 , we outline the classification of irreducible reductive dual pairs. In section 2.3 , we introduce the concept of seesaw pairs, which will be crucial in the rest of the paper.

In section 3, we derive the oscillator realizations of all real forms of the complex Lie group duality $\left(G L_{M}, G L_{N}\right)$, that is, $(G L(M, \mathbb{R}), G L(N, \mathbb{R})),(G L(M, \mathbb{C}), G L(N, \mathbb{C}))$, $\left(U^{*}(2 M), U^{*}(2 N)\right)$ and $\left(U\left(M_{+}, M_{-}\right), U\left(N_{+}, N_{-}\right)\right)$dualities, starting from the definitions of the real forms.

In section 4 , we derive the oscillator realizations of all real forms of the complex Lie group duality $\left(O_{N}, S p_{2 M}\right)$, that is, $\left(O\left(N_{+}, N_{-}\right), S p(2 M, \mathbb{R})\right),(O(N, \mathbb{C}), S p(2 M, \mathbb{C}))$ and $\left(O^{*}(2 N), S p\left(M_{+}, M_{-}\right)\right)$dualities, starting from the definitions of the real forms.

In section 5, we derive the correspondence for "compact dual pairs", i.e. the dual pairs in which at least one member is compact. In doing so, we will recover the familiar oscillator realizations of finite-dimensional representations of compact Lie groups, as well as the lowest weight representations of non-compact Lie groups. We also briefly comment on the application of this correspondence to $\mathrm{AdS}_{d+1} / \mathrm{CFT}_{d}$ for $d=3,4$ and 6 (which will be discussed in more details in a follow-up paper).

In section 6 , we derive the correspondence between representations of what will be referred to as "exceptionally compact pairs", i.e. dual pairs in which one member becomes either compact or discrete due to an exceptional isomorphism. We briefly comment on its role in $\mathrm{dS}_{d}$ representations for $d=3$ and 4 .

In section 7 , we derive the correspondence between representations of dual pairs where both groups are non-compact, but one is Abelian or simple enough. These cases are different from the compact ones in that the representations are of non-polynomial excitations type. We also present the Schrödinger realizations for these representations (i.e. their realizations on $\mathcal{L}^{2+\epsilon}$ spaces).

In section 8 , we discuss interesting aspects of the branching rules of the representations appearing in the dual pair correspondence. We comment on the special cases where one of the groups is the three- or four-dimensional conformal group. In such cases, these representations are known as "singletons" and correspond to free conformal fields.

In section 9, we derive the relation between the Casimir operators (of arbitrary order) of the two groups of a dual pair.

We leave concluding remarks for the sequel papers. 
In order to be as self-contained as possible, we included three appendices detailing textbook material, used in the bulk of the paper, as well as one detailing the derivations of the Casimir relations: In appendix A, we review the realization of finite-dimensional representations of the compact form of the classical groups on spaces of tensors. In appendix B, we summarize the definition of the various real forms of the classical groups. In appendix $\mathrm{C}$, we provide the seesaw pair diagrams involving maximal compact subgroups and their dual for all irreducible dual pairs. Finally, appendix D contains the technical details on the derivations of the Casimir relations.

\subsection{Conventions}

Classical Lie groups and their real forms. We denote the complex classical Lie groups by $G L_{N}, O_{N}$ and $S p_{2 N}$, i.e. the general linear, the orthogonal and the symplectic group, respectively. When these Lie groups are viewed as real Lie groups, they are denoted by $G L(N, \mathbb{C}), O(N, \mathbb{C})$ and $S p(2 N, \mathbb{C})$. The real forms of $G L_{N}$ and $O_{N}$ will be denoted by $G L(N, \mathbb{R}), U^{*}(2 N), U\left(N_{+}, N_{-}\right), O\left(N_{+}, N_{-}\right), O(N, \mathbb{C})$ and $O^{*}(2 N)$ with standard definitions and notations. There is no standard notation for the symplectic groups; we use the notation $S p(2 N, \mathbb{R}), S p(2 N, \mathbb{C})$ and $S p\left(N_{+}, N_{-}\right)$for the groups with rank $N, 2 N$ and $N_{+}+N_{-}$, respectively. See appendix B for the details. The multiplicative group of nonzero real, complex and quaternionic numbers are denoted by $\mathbb{R}^{\times}, \mathbb{C}^{\times}$and $\mathbb{H}^{\times}$, whereas the additive group of real numbers, isomorphic to the multiplicative group of strictly positive real numbers, is denoted by $\mathbb{R}^{+}$. For brevity, the phrase "generators of the Lie group" (also in symbolic form) will be used when actually referring to generators of the associated Lie algebra.

Young diagrams. A Young diagram of the form,

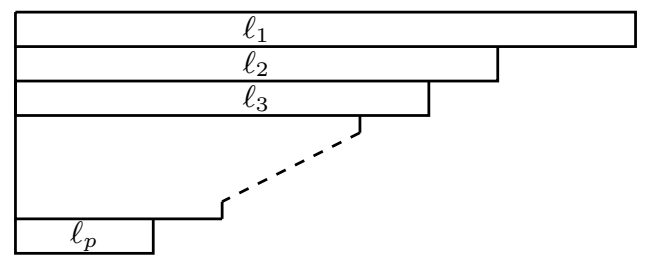

will be denoted by

$$
\ell=\left(\ell_{1}, \ldots, \ell_{p}\right),
$$

i.e. $\ell_{k} \in \mathbb{N}$ corresponds to the length of the $k$-th row of the diagram and $p$ is the number of rows. It will sometimes be useful to present a Young diagram in terms of its columns instead of its rows. In this case, we use the same bold symbol $\ell$, but write its components within square brackets and with upper indices, i.e.

$$
\ell=\left[\ell^{1}, \ldots, \ell^{q}\right]
$$

where $\ell^{k} \in \mathbb{N}$ corresponds to the height of the $k$-th column, and $q$ is the number of columns. Clearly, the two descriptions are equivalent. In particular, $q=\ell_{1}$ and $p=\ell^{1}$. For instance, 
the diagram,

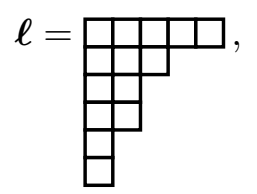

can be presented as

$$
\ell=(5,3,2,2,1,1) \quad \text { and } \quad \ell=[6,4,2,1,1]
$$

Tensors and indices. We will use round and square brackets for symmetrization and antisymmetrization of indices, respectively. For instance,

$$
T^{\left(a_{1} a_{2}\right)}=\frac{1}{2}\left(T^{a_{1} a_{2}}+T^{a_{2} a_{1}}\right), \quad T^{\left[a_{1} a_{2}\right]}=\frac{1}{2}\left(T^{a_{1} a_{2}}-T^{a_{2} a_{1}}\right),
$$

and more generally

$$
T^{\left(a_{1} \ldots a_{n}\right)}=\frac{1}{n !} \sum_{\sigma \in \mathcal{S}_{n}} T^{a_{\sigma(1)} \ldots a_{\sigma(n)}}, \quad T^{\left[a_{1} \ldots a_{n}\right]}=\frac{1}{n !} \sum_{\sigma \in \mathcal{S}_{n}} \operatorname{sgn}(\sigma) T^{a_{\sigma(1)} \ldots a_{\sigma(n)}},
$$

where $\operatorname{sgn}(\sigma)$ is the signature of the permutation $\sigma$, which is equal to +1 if $\sigma$ is an even permutation and -1 if it is odd.

Given a non-degenerate antisymmetric matrix $\Omega$ with components satisfying

$$
\Omega_{A B}=-\Omega_{B A}, \quad \Omega_{A C} \Omega^{C B}=\delta_{A}^{B},
$$

the indices of $S p_{2 N}$ tensors will be raised and lowered as follows

$$
T^{A}=\Omega^{A B} T_{B}, \quad T_{A}=\Omega_{A B} T^{B} .
$$

In particular, this implies that

$$
\Omega_{A B} V^{A} W^{B}=V^{A} W_{A}=-V_{A} W^{A} .
$$

Oscillators. In this paper, annihilation operators will be denoted by $a_{A}$ or $a_{A}^{I}$ with one or two indices. Their Hermitian conjugate, i.e. the creation operators, will be denoted with a tilde and opposite index position, i.e.

$$
\left(a_{A}^{I}\right)^{\dagger}=\tilde{a}_{I}^{A}
$$

We will refer to the creation and annihilation operators as oscillators. The letters $b, c$ and $d$ will also be used to denote (additional) oscillators.

Representations. A generic irreducible representation of a Lie group $G$ will be denoted by

$$
\pi_{G}\left(\zeta_{1}, \ldots, \zeta_{p}\right)
$$


where $\zeta_{1}, \ldots, \zeta_{p}$ are the (continuous or discrete) parameters which label the representation. ${ }^{6}$ For two particular types of representations, the following specific notation will be used:

- Finite-dimensional irreducible representations of the (double-cover of the) compact Lie groups $G=U(N), O(N)$ or $S p(N)$, will be denoted by $[\boldsymbol{\ell}, \delta]_{G}$, where $\boldsymbol{\ell}$ is a Young diagram, and $\delta \in \frac{1}{2} \mathbb{Z}$ is a half-integer such that

$$
\left(\ell_{1}+\delta, \ldots, \ell_{N}+\delta\right)
$$

is the highest weight of the representation. Here, $\ell_{p+1}=\cdots=\ell_{N}=0$ for the Young diagram $\ell$ with height $p$. The correspondence between these highest weight representations and tensors with symmetry of the Young diagram $\ell$ is reviewed in appendix A.

- Infinite-dimensional irreducible representations of a non-compact Lie group $G$ of lowest weight type will be denoted by

$$
\mathcal{D}_{G}\left(\ell_{1}, \ldots, \ell_{\operatorname{rank}(G)}\right),
$$

where $\ell_{k}$ are the components of the lowest weight defining the representation. If the group $G$ has the same rank as its maximal compact subgroup $K$, we will combine this notation with the previous one as

$$
\mathcal{D}_{G}\left([\ell, \delta]_{K}\right) .
$$

\section{Generalities of the reductive dual pair correspondence}

A reductive dual pair $\left(G, G^{\prime}\right) \subset S p(2 N, \mathbb{R})$ consists of two subgroups, $G$ and $G^{\prime}$, of $S p(2 N, \mathbb{R})$ which are centralizers of each other and act reductively on $\mathbb{R}^{2 N}$, meaning that they consist of those elements in $S p(2 N, \mathbb{R})$, which reduce completely $\mathbb{R}^{2 N}$ into irreducible parts invariant under the subgroup elements. In other words, when realized as $2 N \times 2 N$ real matrices, reductive subgroups are those having block-diagonal elements, with each block being an irreducible representation of the subgroup. (Recall that $S p(2 N, \mathbb{R})$ is the group of linear transformations of $\mathbb{R}^{2 N}$ preserving the symplectic form $\Omega_{A B} x^{A} y^{B}$, and hence the space $\mathbb{R}^{2 N}$ carries the defining representation of $S p(2 N, \mathbb{R})$.)

A central result due to Howe $[1,2]$ is that the restriction of the metaplectic representation $\mathcal{W}$ of $S p(2 N, \mathbb{R})$, to be discussed below, to $G \times G^{\prime}$ establishes a bijection between representations of $G$ and those of $G^{\prime}$ appearing in the decomposition of $\mathcal{W}$. More precisely, $\mathcal{W}$ can be decomposed as

$$
\left.\mathcal{W}\right|_{G \times G^{\prime}}=\bigoplus_{\zeta \in \Sigma_{\mathcal{W}}^{G}} \pi_{G}(\zeta) \otimes \pi_{G^{\prime}}(\theta(\zeta)),
$$

\footnotetext{
${ }^{6}$ In general, a representation of $G$ can be induced from a representation of one of its parabolic subgroups $P$. For this reason, a generic irreducible representation is usually denoted by

$$
\operatorname{Ind}_{P}^{G}\left(\zeta_{1}, \ldots, \zeta_{p}\right)
$$

The parameters $\zeta_{i}$ indicate a representation of $P$ which induces the representation of $G$. The couple $P$ and $\left(\zeta_{1}, \ldots, \zeta_{p}\right)$ are called the Langlands parameters.
} 
where $\pi_{G}(\zeta)$ and $\pi_{G^{\prime}}(\theta(\zeta))$ are irreducible representations (irreps) of $G$ and $G^{\prime}$ labeled by $\zeta$ and $\theta(\zeta)$, respectively. The map,

$$
\begin{aligned}
\theta: \Sigma_{\mathcal{W}}^{G} & \longrightarrow \Sigma_{\mathcal{W}}^{G^{\prime}}, \\
\zeta & \longmapsto \theta(\zeta),
\end{aligned}
$$

defines a bijection between a set $\Sigma_{\mathcal{W}}^{G}$ of $G$ irreps and the corresponding set $\Sigma_{\mathcal{W}}^{G^{\prime}}$ of $G^{\prime}$ irreps. In other words, each $G$ representation appears only once in $\Sigma_{\mathcal{W}}^{G}$ and is paired with a unique $G^{\prime}$ representation in $\Sigma_{\mathcal{W}}^{G^{\prime}}$, and vice-versa. As a consequence, the representation $\pi_{G}(\zeta)$ occurs with multiplicity $\operatorname{dim} \pi_{G^{\prime}}(\theta(\zeta))$, and similarly for $\pi_{G^{\prime}}(\theta(\zeta))$ :

$$
\operatorname{mult}_{\mathcal{W}}\left(\pi_{G}(\zeta)\right)=\operatorname{dim} \pi_{G^{\prime}}(\theta(\zeta)), \quad \operatorname{mult}_{\mathcal{W}}\left(\pi_{G^{\prime}}(\theta(\zeta))\right)=\operatorname{dim} \pi_{G}(\zeta) .
$$

More details can be found, e.g. in the review articles [28, 70], or in the textbooks [71, 72] (where Howe duality is presented within the broader context of duality in representation theory).

\subsection{Metaplectic representation}

The metaplectic representation $\mathcal{W}$ of $\operatorname{Sp}(2 N, \mathbb{R})$ is known under different names, such as the harmonic representation, the Segal-Shale-Weil representation or simply the oscillator representation [23-25]. This representation can be realized as a Fock space, which is generated by the free action of creation operators $a_{i}^{\dagger}$ with $i=1, \ldots, N$ on a vacuum state $|0\rangle$, which, by definition, is annihilated by the annihilation operators $a_{i}$ :

$$
\mathcal{W}=\operatorname{span}_{\mathbb{C}}\left\{a_{i_{1}}^{\dagger} \ldots a_{i_{k}}^{\dagger}|0\rangle, k \in \mathbb{N}\right\}, \quad\left[a_{i}, a_{j}^{\dagger}\right]=\delta_{i j}, \quad a_{i}|0\rangle=0 .
$$

This infinite-dimensional space carries an irreducible and unitary representation of the order $N$ Heisenberg algebra, whose generators are represented by the $N$ pairs of creation and annihilation operators, as well as the identity (representing its center). Moreover, the operators bilinear in $a_{i}$ and $a_{i}^{\dagger}$ provide a representation of the Lie algebra $\mathfrak{s p}(2 N, \mathbb{R})$ of the symplectic group $S p(2 N, \mathbb{R})$. More precisely, the operators,

$$
K^{i j}=a_{i}^{\dagger} a_{j}^{\dagger}, \quad K^{i}{ }_{j}=\frac{1}{2}\left\{a_{i}^{\dagger}, a_{j}\right\}=a_{i}^{\dagger} a_{j}+\frac{1}{2} \delta_{i j}, \quad K_{i j}=-a_{i} a_{j},
$$

satisfy the commutation relations of $\mathfrak{s p}(2 N, \mathbb{R})$, i.e.

$$
\begin{aligned}
{\left[K^{i}{ }_{j}, K^{k}{ }_{l}\right] } & =\delta_{j}^{k} K^{i}{ }_{l}-\delta_{l}^{i} K^{k}{ }_{j}, & & {\left.\left[K^{i j}, K_{k l}\right]=4 \delta_{(k}^{(i} K^{j)} l\right), } \\
{\left[K^{i}{ }_{j}, K_{k l}\right] } & =-2 \delta_{(k}^{i} K_{l) j}, & {\left[K^{i}{ }_{j}, K^{k l}\right] } & =2 \delta_{j}^{(k} K^{l) i} .
\end{aligned}
$$

In this basis, the operators $K_{i}^{i}$ (where no summation is implied) generate a Cartan subalgebra while the operators $K_{i j}$ and $K_{l}^{k}$ with $k<l$ correspond to lowering operators, and the remaining ones to raising operators. The metaplectic representation is a direct sum of two $\mathfrak{s p}(2 N, \mathbb{R})$ lowest weight representations,

$$
\mathcal{W}=\mathcal{D}_{S p(2 N, \mathbb{R})}\left(\frac{1}{2}, \frac{1}{2}, \ldots, \frac{1}{2}\right) \oplus \mathcal{D}_{S p(2 N, \mathbb{R})}\left(\frac{3}{2}, \frac{1}{2}, \ldots, \frac{1}{2}\right) .
$$

The vacuum $|0\rangle$ and $a_{1}^{\dagger}|0\rangle$ are the lowest weight vectors of the above two representations with weight $\left(\frac{1}{2}, \frac{1}{2}, \ldots, \frac{1}{2}\right)$ and $\left(\frac{3}{2}, \frac{1}{2}, \ldots, \frac{1}{2}\right)$, respectively. ${ }^{7}$ Repeated action of rais-

\footnotetext{
${ }^{7}$ The seemingly privileged role of $a_{1}^{\dagger}$ comes from the choice of lowering operators, $K^{k}{ }_{l}$ with $k<l$.
} 
ing operators on these vectors makes up the representations $\mathcal{D}_{S p(2 N, \mathbb{R})}\left(\frac{1}{2}, \frac{1}{2}, \ldots, \frac{1}{2}\right)$ and $\mathcal{D}_{S p(2 N, \mathbb{R})}\left(\frac{3}{2}, \frac{1}{2}, \ldots, \frac{1}{2}\right)$, which are therefore composed of the states with even and odd excitation numbers, respectively.

The metaplectic representation can also be decomposed in terms of the maximal compact subalgebra $\mathfrak{u}(N) \cong \mathfrak{u}(1) \oplus \mathfrak{s u}(N)$, where the $\mathfrak{u}(1)$ and $\mathfrak{s u}(N)$ subalgebras are generated respectively by $K^{i}{ }_{i}=\sum_{k=1}^{N} a_{k}^{\dagger} a_{k}+\frac{N}{2}$ and $K^{i}{ }_{j}-\frac{1}{N} \delta_{j}^{i} K^{k}{ }_{k}=a_{j}^{\dagger} a_{i}-\frac{1}{N} \delta_{j}^{i} \sum_{k=1}^{N} a_{k}^{\dagger} a_{k}$. The vacuum $|0\rangle$ carries the irrep $\left[(0), \frac{1}{2}\right]_{U(N)}$ (i.e. it has the $\mathfrak{u}(1)$ eigenvalue $\frac{N}{2}$ and carries the trivial representations of $\mathfrak{s u}(N))$, whereas the $N$ dimensional space generated by $a_{i}^{\dagger}|0\rangle$ carries the irrep $\left[(1), \frac{1}{2}\right]_{U(N)}$ (i.e. it has the $\mathfrak{u}(1)$ eigenvalue $\frac{N}{2}+1$ and carries the fundamental representation of $\mathfrak{s u}(N))$. These $\mathfrak{u}(N)$ representations are annihilated by the lowering operators $K_{i j}$ and they induce the $\mathfrak{s p}(2 N, \mathbb{R})$ irreps $\mathcal{D}_{S p(2 N, \mathbb{R})}\left(\left[(0), \frac{1}{2}\right]_{U(N)}\right)=$ $\mathcal{D}_{S p(2 N, \mathbb{R})}\left(\frac{1}{2}, \frac{1}{2}, \ldots, \frac{1}{2}\right)$, and $\mathcal{D}_{S p(2 N, \mathbb{R})}\left(\left[(1), \frac{1}{2}\right]_{U(N)}\right)=\mathcal{D}_{S p(2 N, \mathbb{R})}\left(\frac{3}{2}, \frac{1}{2}, \ldots, \frac{1}{2}\right)$ by the action of the raising operators $K^{i j}$.

At this point, we have seen that $\mathcal{W}$ carries both a representation of the order $N$ Heisenberg algebra and of the symplectic algebra $\mathfrak{s p}(2 N, \mathbb{R})$. A natural question is then to ask whether this can be uplifted to a group representation in both cases. For the Heisenberg group, the answer is positive: a direct computation shows that the operators $U(\alpha, \beta, \gamma):=e^{\alpha^{i} a_{i}+\beta^{i} a_{i}^{\dagger}+\gamma}$ satisfy

$$
U\left(\alpha_{1}, \beta_{1}, \gamma_{1}\right) U\left(\alpha_{2}, \beta_{2}, \gamma_{2}\right)=U\left(\alpha_{1}+\alpha_{2}, \beta_{1}+\beta_{2}, \gamma_{1}+\gamma_{2}+\frac{1}{2}\left(\alpha_{1} \cdot \beta_{2}-\beta_{1} \cdot \alpha_{2}\right)\right)
$$

where $\alpha \cdot \beta=\sum_{i=1}^{N} \alpha^{i} \beta^{i}$. The above reproduces the group multiplication of the Heisenberg group $H_{N}$ and hence provides its representation on $\mathcal{W}$.

The situation is a bit more subtle for the symplectic group. It turns out that the exponentiation of the $\mathfrak{s p}(2 N, \mathbb{R})$ representation $(2.5)$ is double-valued. To see whether a representation is single-valued or not, we need to examine the representation along a loop in the group manifold. Since the loops contractible to a point will always give single-valued representations, we must consider only the other kind of loops, which are classified by the fundamental group of the group manifold. The fundamental group of $\operatorname{Sp}(2 N, \mathbb{R})$ is that of its maximal compact subgroup $U(N) \cong U(1) \ltimes S U(N)$, where the $U(1)$ can be chosen to have an element $\operatorname{diag}(e^{i \phi}, \underbrace{1, \ldots, 1}_{N-1}){ }^{8}$ This element of $U(1)$ is embedded in $S p(2 N, \mathbb{R})$ as

$$
g(\phi)=\left(\begin{array}{cccc}
\cos \phi & 0 & \sin \phi & 0 \\
0 & I_{N-1} & 0 & I_{N-1} \\
-\sin \phi & 0 & \cos \phi & 0 \\
0 & I_{N-1} & 0 & I_{N-1}
\end{array}\right) .
$$

Since $S U(N)$ has a trivial first fundamental group, we can just consider this $U(1)$ subgroup, generated by

$$
K_{1}^{1}=a_{1}^{\dagger} a_{1}+\frac{1}{2},
$$

\footnotetext{
${ }^{8}$ As a topological space, $U(N)$ is homeomorphic to the product space $U(1) \times S U(N)$, where the $U(1)$ subgroup cannot be taken as the diagonal one, $U(1)_{\text {diag }}=\left\{e^{i \phi} I_{N} \mid 0 \leq \phi<2 \pi\right\}$. In fact, as a group, $U(N) \cong\left(U(1)_{\operatorname{diag}} \times S U(N)\right) / \mathbb{Z}_{N}$ where $\mathbb{Z}_{N}=\left\{e^{i 2 \pi \frac{n}{N}} I_{N} \mid n=1, \ldots, N-1\right\}$.
} 
and exponentiating $K_{1}^{1}$, one finds the representation,

$$
U_{\mathcal{W}}(\phi)=e^{i \phi K^{1} 1}=e^{i \phi\left(a_{1}^{\dagger} a_{1}+\frac{1}{2}\right)} .
$$

Since $a_{1}^{\dagger} a_{1}$ has integer eigenvalues, the $U(1)$ representation (2.12) has the property,

$$
U_{\mathcal{W}}(\phi+2 \pi)=-U_{\mathcal{W}}(\phi),
$$

that is, the representation map $U_{\mathcal{W}}$ is double-valued. One can replace the $U(1)$ subgroup by its double cover to render the representation map $U_{\mathcal{W}}$ single-valued. This would require to replace the symplectic group $S p(2 N, \mathbb{R})$ by its double cover, namely the metaplectic group, often denoted by $\widetilde{S p}(2 N, \mathbb{R})$. In principle, of course, one can also consider an arbitrary even cover of $S p(2 N, \mathbb{R})$.

As we discussed just above, the essential point resides in the $U(1)$ part of $S p(2 N, \mathbb{R})$, but it will still be instructive to consider the action of the entire $S p(2 N, \mathbb{R})$ group. For simplicity, let us focus on the $N=1$ case, $S p(2, \mathbb{R})$, whose element can be parameterized as

$$
g(\phi, \psi, \tau)=\left(\begin{array}{cc}
\cos \phi \cosh \tau-\sin \psi \sinh \tau & \sin \phi \cosh \tau+\cos \psi \sinh \tau \\
-\sin \phi \cosh \tau+\cos \psi \sinh \tau & \cos \phi \cosh \tau+\sin \psi \sinh \tau
\end{array}\right),
$$

with $g(\phi+2 \pi, \psi, \tau)=g(\phi, \psi, \tau)$ and $g(\phi, \psi+\pi, \tau)=g(\phi, \psi,-\tau)$ and $\tau \in \mathbb{R}$. The oscillator representation for the group element $g(\phi, \psi, \tau)$ can be obtained again by exponentiation and expressed as

$$
U_{\mathcal{W}}(\phi, \psi, \tau)=e^{i \phi\left(a^{\dagger} a+\frac{1}{2}\right)} e^{\beta a^{2}-\beta^{*}\left(a^{\dagger}\right)^{2}},
$$

where $\beta=\frac{1}{2} e^{i(\psi-\phi)} \tau$. Again, one can check the double-valued-ness of the map $U_{\mathcal{W}}$ :

$$
U_{\mathcal{W}}(\phi+2 \pi, \psi, \tau)=-U_{\mathcal{W}}(\phi, \psi, \tau) .
$$

We may avoid this problem by viewing $U_{\mathcal{W}}$ as a representation of the double cover $\widetilde{S p}(2, \mathbb{R})$; it is parametrized by the same variables as $S p(2, \mathbb{R})$, but the period of $\phi$ is extended to $4 \pi$. Since $U_{\mathcal{W}}$ is faithful for the double cover (but not for other even covers) of $S p(2, \mathbb{R})$, it can serve as a definition of $\widetilde{S p}(2, \mathbb{R})$, which does not admit any matrix realization. Instead of considering the double cover, one may also try to cure the problem (2.16) by modifying the representation to

$$
V_{\mathcal{W}}(\phi, \psi, \tau)=e^{i \phi a^{\dagger} a} e^{\beta a^{2}-\beta^{*}\left(a^{\dagger}\right)^{2}}=U_{\mathcal{W}}(\phi, \psi, \tau) e^{-\frac{i}{2} \phi},
$$

which is single-valued. But, then the above has a modified composition rule,

$$
V_{\mathcal{W}}\left(\phi_{1}, \psi_{1}, \tau_{1}\right) V_{\mathcal{W}}\left(\phi_{2}, \psi_{2}, \tau_{2}\right)=e^{\frac{i}{2}\left(\phi_{3}-\phi_{1}-\phi_{2}\right)} V_{\mathcal{W}}\left(\phi_{3}, \psi_{3}, \tau_{3}\right),
$$

where $\phi_{3}, \psi_{3}, \tau_{3}$ satisfy $g\left(\phi_{1}, \psi_{1}, \tau_{1}\right) g\left(\phi_{2} \psi_{2} \tau_{2}\right)=g\left(\phi_{3}, \psi_{3}, \tau_{3}\right)$. Therefore, $V_{\mathcal{W}}$ is a projective representation of $S p(2, \mathbb{R})$.

A more standard way to introduce the metaplectic representation is as follows: given a pair of creation and annihilation operators $\left(a^{\dagger}, a\right)$, one can define a new pair $\left(b^{\dagger}, b\right)$ through a Bogoliubov transformation, which is simply a linear transformation,

$$
\left(\begin{array}{c}
b \\
b^{\dagger}
\end{array}\right)=A\left(\begin{array}{c}
a \\
a^{\dagger}
\end{array}\right)
$$


preserving the canonical commutation relation, $\left[b, b^{\dagger}\right]=1$. The latter condition implies that $A \in S U(1,1)$, which can be parametrized by

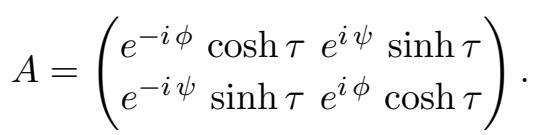

Since $S U(1,1) \cong S p(2, \mathbb{R})$, the two-dimensional symplectic group $S p(2, \mathbb{R})$ is isomorphic to the center-preserving automorphism group $S U(1,1)$ of the Heisenberg algebra. Therefore, the Bogoliubov transformation (2.19) defines an action of $S p(2, \mathbb{R})$ on $\mathcal{W}$, which can be expressed as

$$
\left(\begin{array}{c}
b \\
b^{\dagger}
\end{array}\right)=U_{\mathcal{W}}(\phi, \psi, \tau)\left(\begin{array}{c}
a \\
a^{\dagger}
\end{array}\right) U_{\mathcal{W}}(\phi, \psi, \tau)^{-1} .
$$

where $U_{\mathcal{W}}$ is given in $(2.15)$, and $A$ and $g(\phi, \psi, \tau)$ in (2.14) are related by

$$
g(\phi, \psi, \tau)=U^{-1} A U, \quad U=\frac{1}{\sqrt{2}}\left(\begin{array}{ll}
1 & i \\
i & 1
\end{array}\right) .
$$

Since the formula (2.21) is invariant under redefinition of $U_{\mathcal{W}}$ with a phase factor, it provides a projective representation of $S p(2, \mathbb{R})$.

To conclude, let us consider the reflection subgroup of $\operatorname{Sp}(2 N, \mathbb{R})$, which will be used in identifying representations of dual pairs. They are contained again in the maximal compact subgroup $U(N)$ as the diagonal elements,

$$
\mathcal{R}_{i}=e^{i \pi K^{i}}=e^{i \pi\left(a_{i}^{\dagger} a_{i}+\frac{1}{2}\right)},
$$

where no summation for the $i$ index is implied. The reflection $\mathcal{R}_{i}$ flips the sign of the $i$-th pair of oscillators:

$$
\mathcal{R}_{i}\left(\begin{array}{c}
a_{i} \\
a_{i}^{\dagger}
\end{array}\right) \mathcal{R}_{i}^{-1}=-\left(\begin{array}{c}
a_{i} \\
a_{i}^{\dagger}
\end{array}\right)
$$

so it has a $\mathbb{Z}_{2}$ action on the oscillators. Notice, however, that the reflection does not form a $\mathbb{Z}_{2}$ group, but a $\mathbb{Z}_{4}$ :

$$
\mathcal{R}_{i}^{2}=-1, \quad \mathcal{R}_{i}^{4}=1
$$

This is because we are considering the metaplectic group where the maximal compact subgroup $U(N)$ should be understood as the double cover of the latter. Since these double cover groups are not matrix groups, "reflections" do not work in the way we know for the matrix group. Viewed as an automorphism group of Heisenberg algebra $H_{N}$, the reflections form $\mathbb{Z}_{2}$ groups. Besides the aforementioned reflections, there are many other $\mathbb{Z}_{2}$ or $\mathbb{Z}_{4}$ automorphism groups of $H_{N}$. Obviously, the permutation group $S_{N}$ lying in the subgroup of $O(N) \subset S p(2 N, \mathbb{R})$ contains many $S_{2} \cong \mathbb{Z}_{2}$ groups, i.e. the permutations of two oscillators:

$$
\mathcal{S}_{i j}\left(\begin{array}{c}
a_{i} \\
a_{i}^{\dagger} \\
a_{j} \\
a_{j}^{\dagger}
\end{array}\right) \mathcal{S}_{i j}{ }^{-1}=\left(\begin{array}{c}
a_{j} \\
a_{j}^{\dagger} \\
a_{i} \\
a_{i}^{\dagger}
\end{array}\right) .
$$


Less obvious $\mathbb{Z}_{2}$ automorphisms are

$$
\mathcal{P}_{i j}\left(\begin{array}{c}
a_{i} \\
a_{i}^{\dagger} \\
a_{j} \\
a_{j}^{\dagger}
\end{array}\right) \mathcal{P}_{i j}{ }^{-1}=\left(\begin{array}{c}
a_{j}^{\dagger} \\
-a_{j} \\
-a_{i}^{\dagger} \\
a_{i}
\end{array}\right),
$$

which can be decomposed as

$$
\mathcal{P}_{i j}=\mathcal{S}_{i j} \mathcal{P}_{i} \mathcal{P}_{j}^{-1}
$$

Here, $\mathcal{P}_{i}$ acts on $H_{N}$ as

$$
\mathcal{P}_{i}\left(\begin{array}{c}
a_{i} \\
a_{i}^{\dagger}
\end{array}\right) \mathcal{P}_{i}^{-1}=\left(\begin{array}{c}
-a_{i}^{\dagger} \\
a_{i}
\end{array}\right),
$$

forming a $\mathbb{Z}_{4}$ automorphism of $H_{N}$, though it is not compatible with the reality structure of $H_{N}$. However, one can still view $\mathcal{P}_{i}$ as $\mathbb{Z}_{2}$ automorphisms of $S p(2 N, \mathbb{R})$ preserving the reality condition of the latter. Basically, it interchanges the raising and lowering operators and flips the signs of all generators of the maximal compact subalgebra $\mathfrak{u}(N)$, and hence corresponds to the composition of the Cartan and Chevalley involutions. From the identity,

$$
e^{i \frac{\theta}{2}\left(\left(a_{i}^{\dagger}\right)^{2}-\left(a_{i}\right)^{2}\right)}\left(\begin{array}{c}
a_{i} \\
a_{i}^{\dagger}
\end{array}\right) e^{-i \frac{\theta}{2}\left(\left(a_{i}^{\dagger}\right)^{2}-\left(a_{i}\right)^{2}\right)}=\left(\begin{array}{cc}
\cos \theta & -\sin \theta \\
\sin \theta & \cos \theta
\end{array}\right)\left(\begin{array}{c}
a_{i} \\
a_{i}^{\dagger}
\end{array}\right),
$$

we can realize $\mathcal{P}_{i}$ as an exponentiation of quadratic oscillators,

$$
\mathcal{P}_{i}=e^{i \frac{\pi}{4}\left(K_{i i}-K^{i i}\right)}=e^{i \frac{\pi}{4}\left(\left(a_{i}^{\dagger}\right)^{2}-\left(a_{i}\right)^{2}\right)} .
$$

Note however that such a realization of $\mathcal{P}_{i}$ is not unitary, and hence they are outerautomorphism of $S p(2 N, \mathbb{R})$. In analyzing dual subgroups of $S p(2 N, \mathbb{R})$, it will be often useful to consider the automorphisms $\mathcal{S}_{i j}, \mathcal{P}_{i j}$ and $\mathcal{P}_{i}$.

As we have discussed, the metaplectic representation can be easily understood using the Fock realization (also referred to as the Fock model). Another simple description of it is the wave function in the configuration space - the Schrödinger realization. The relation between oscillator realization and wave function realization is nothing but the typical relation between $\left(\hat{x}_{i}, \hat{p}_{i}\right)$ and $\left(a_{i}, a_{i}^{\dagger}\right)$ of the quantum harmonic oscillator,

$$
\hat{x}_{i}=\frac{1}{\sqrt{2}}\left(a_{i}+a_{i}^{\dagger}\right), \quad \hat{p}_{i}=\frac{i}{\sqrt{2}}\left(a_{i}^{\dagger}-a_{i}\right) .
$$

Even though the above relation is a very familiar one, it helps to simplify the description of representations in several cases: a certain complicated state $|\Psi\rangle$ in the Fock space becomes simple as wave function $\langle x \mid \Psi\rangle$, and vice-versa. Another useful description is provided by the Bargmann-Segal realization, wherein the states are holomorphic functions in $\mathbb{C}^{N}$, which are square integrable with respect to a Gaussian measure. In this realization, the creation operators simply act as multiplication $z$ while the annihilation operators act as derivatives with respect to these variables, i.e.

$$
a_{i}^{\dagger}=z_{i}, \quad a_{i}=\frac{\partial}{\partial z_{i}} .
$$

The connection to the Schrödinger realization is made by an integral transform, called the Bargmann-Segal transform [73, 74]. 


\begin{tabular}{|c|c|c|}
\hline Embedding group & $\left(G, G^{\prime}\right)$ & $\left(K, K^{\prime}\right)$ \\
\hline$S p(2 M N, \mathbb{R})$ & $(G L(M, \mathbb{R}), G L(N, \mathbb{R}))$ & $(O(M), O(N))$ \\
\hline$S p(4 M N, \mathbb{R})$ & $(G L(M, \mathbb{C}), G L(N, \mathbb{C}))$ & $(U(M), U(N))$ \\
\hline$S p(8 M N, \mathbb{R})$ & $\left(U^{*}(2 M), U^{*}(2 N)\right)$ & $(M), S p(N))$ \\
\hline$S p\left(2\left(M_{+}+M_{-}\right)\left(N_{+}+N_{-}\right), \mathbb{R}\right)$ & $\left(U\left(M_{+}, M_{-}\right), U\left(N_{+}, N_{-}\right)\right)$ & $\left(U\left(M_{+}\right) \times U\left(M_{-}\right), U\left(N_{+}\right) \times U\left(N_{-}\right)\right)$ \\
\hline$S p\left(2 M\left(N_{+}+N_{-}\right), \mathbb{R}\right)$ & $\left(O\left(N_{+}, N_{-}\right), S p(2 M, \mathbb{R})\right)$ & $\left(O\left(N_{+}\right) \times O\left(N_{-}\right), U(M)\right)$ \\
\hline$S p(4 M N, \mathbb{R})$ & $(O(N, \mathbb{C}), S p(2 M, \mathbb{C}))$ & $(O(N), S p(M))$ \\
\hline$S p\left(4 N\left(M_{+}+M_{-}\right), \mathbb{R}\right)$ & $\left(O^{*}(2 N), S p\left(M_{+}, M_{-}\right)\right)$ & $\left(U(N), S p\left(M_{+}\right) \times S p\left(M_{-}\right)\right)$ \\
\hline
\end{tabular}

Table 1. List of all possible irreducible reductive dual pairs $\left(G, G^{\prime}\right)$ embedded in metaplectic groups and their respective maximal compact subgroups $\left(K, K^{\prime}\right)$.

\subsection{Irreducible reductive dual pairs}

For a given embedding group $S p(2 N, \mathbb{R})$, we can find many inequivalent dual pairs. Any reductive dual pair $\left(G, G^{\prime}\right)$ has the form,

$$
G=G_{1} \times G_{2} \times \cdots \times G_{p}, \quad G^{\prime}=G_{1}^{\prime} \times G_{2}^{\prime} \times \cdots \times G_{p}^{\prime},
$$

where each couple $\left(G_{k}, G_{k}^{\prime}\right)$ is one of the irreducible dual pairs listed in table 1 and embedded in a symplectic group $S p\left(2 N_{k}, \mathbb{R}\right)$ such that $N_{1}+\cdots+N_{p}=N$. Moreover, one can distinguish between two types of dual pairs: the first one is a real form of $\left(G L_{M}, G L_{N}\right) \subset$ $S p(2 M N, \mathbb{R})$ while the second one is a real form of $\left(O_{N}, S p_{2 M}\right) \subset S p(2 M N, \mathbb{R})$. For instance, in $S p(2 N, \mathbb{R})$ one can find the irreducible pair $(U(N), U(1))$, which is an example of the first type, or the irreducible pair $(O(N), S p(2, \mathbb{R}))$, which is an example of the second type. Accordingly, the pair $(O(N-m) \times U(m), S p(2, \mathbb{R}) \times U(1))$ is an example of a reducible dual pair in $S p(2 N, \mathbb{R})$ for $m=1, \ldots, N$.

Often irreducible dual pairs are classified, using another criterion, again into two types: the type II if there exists a Lagrangian subspace in $\mathbb{R}^{2 N}$ left invariant under both of $G$ and $G^{\prime}$, and the type I otherwise. In the above table, the first three cases are type II, while the rest are type I.

We can extend the idea of the dual pair correspondence to other groups / representations than the symplectic group / metaplectic representation. For instance, one can also consider supersymmetric dual pairs embedded in $O S p(N \mid 2 M, \mathbb{R}$ ) (see e.g. [72, 75] and references therein). Another possible extension is to replace the symplectic group with any simple group $G$ and the metaplectic representation with the minimal representation. ${ }^{9}$ The classification of such pairs was obtained in [77] (see also $[78,79]$ for complementary work).

\subsection{Seesaw pairs}

A useful concept is that of a seesaw pair introduced in [80] (reviewed e.g. in [26, 28]). Consider the situation wherein we have two reductive dual pairs $\left(G, G^{\prime}\right)$ and $\left(\tilde{G}, \tilde{G}^{\prime}\right)$ in

\footnotetext{
${ }^{9}$ The minimal representation of a simple group $G$ is the representation whose annihilator in the universal enveloping algebra $\mathcal{U}(\mathfrak{g})$ is the Joseph ideal, which is the maximal primitive and completely prime ideal of $\mathcal{U}(\mathfrak{g})$ (see e.g. $[60,76])$.
} 
the same symplectic group, say $S p(2 N, \mathbb{R})$. If the groups forming the second pair $\left(\tilde{G}, \tilde{G}^{\prime}\right)$ satisfy $\tilde{G} \subset G$ and $G^{\prime} \subset \tilde{G}^{\prime}$, then the pair of dual pairs $\left(G, G^{\prime}\right)$ and $\left(\tilde{G}, \tilde{G}^{\prime}\right)$ is called a "seesaw pair", and the situation is depicted as

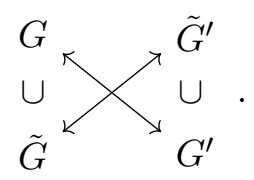

Seesaw pairs satisfy the property,

$$
\operatorname{Hom}_{\tilde{G}}\left(\pi_{\tilde{G}},\left.\pi_{G}\right|_{\tilde{G}}\right) \cong \operatorname{Hom}_{G^{\prime}}\left(\pi_{G^{\prime}},\left.\pi_{\tilde{G}^{\prime}}\right|_{G^{\prime}}\right),
$$

which means the space of $\tilde{G}$-equivariant linear maps (or intertwiners) from $\pi_{\tilde{G}}$ to $\left.\pi_{G}\right|_{\tilde{G}}$, namely $\operatorname{Hom}_{\tilde{G}}\left(\pi_{\tilde{G}},\left.\pi_{G}\right|_{\tilde{G}}\right)$, is isomorphic to the space of $G^{\prime}$-equivariant linear maps from $\pi_{G^{\prime}}$ to $\left.\pi_{\tilde{G}^{\prime}}\right|_{G^{\prime}}$, namely $\operatorname{Hom}_{G^{\prime}}\left(\pi_{G^{\prime}},\left.\pi_{\tilde{G}^{\prime}}\right|_{G^{\prime}}\right)$. Here, $\left.\right|_{\tilde{G}}$ (resp. $\left.\right|_{G^{\prime}}$ ) denotes the restriction to $\tilde{G}$ (resp. $\left.G^{\prime}\right)$. In particular, this implies

$$
\operatorname{mult}_{\pi_{G}}\left(\pi_{\tilde{G}}\right)=\operatorname{mult}_{\pi_{\tilde{G}^{\prime}}}\left(\pi_{G^{\prime}}\right)
$$

i.e. the multiplicity of the $\tilde{G}$-representation $\pi_{\tilde{G}}$ in the branching rule of $\pi_{G}$ is the same as the multiplicity of its dual $\pi_{G^{\prime}}$ in the branching rule of the $\tilde{G}^{\prime}$-representation $\pi_{\tilde{G}^{\prime}}$. This situation is usually depicted as

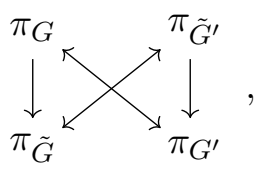

where the downward arrows denote the restriction of a $G$-irrep (resp. $\tilde{G}^{\prime}$-irrep) to a $\tilde{G}$-irrep (resp. $G^{\prime}$-irrep). Seesaw pairs are particularly useful when trying to derive the explicit correspondence between representations of $G$ and $G^{\prime}$, assuming that the correspondence is known for representations of the pair $\left(\tilde{G}, \tilde{G}^{\prime}\right)$, or to obtain the decomposition of a representation into its maximal compact subgroup.

Let us illustrate this with a simple example. Both $(S p(2, \mathbb{R}), O(2))$ and $(U(1), U(2))$ are dual pairs in $S p(4, \mathbb{R})$, which form a seesaw pair:

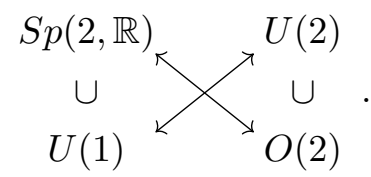

Note that the generator of the $O(2)$ subgroup of $U(2)$ correspond to the $U(1)$ generator of $S U(2)$, and not that of the diagonal $U(1)$. We will see later in section 5 the following correspondences,

$$
\mathcal{D}_{S p(2, \mathbb{R})}(\ell+1) \nearrow_{[m+1]_{U(1)}}^{[m+1]_{U(1)} \otimes[m]_{S U(2)}},
$$


where $\ell$ and $m$ are non-negative integers. We can use the seesaw pair property to find the restriction of $\mathcal{D}_{S p(2, \mathbb{R})}(\ell+1)$ under its maximal compact subgroup $U(1)$ : these representations will be dual to the $U(2)$ representations whose restriction under $O(2)$ contains $[\ell]_{O(2)}$. The branching rule $U(2) \downarrow O(2)$ amounts to finding those $S U(2)$ irreps which contain a pair of states with weight $\pm \ell$. As the irrep $[m]_{S U(2)}$ contains states with weight $k=-m,-m+2, \ldots, m$, we can conclude that the relevant $U(2)$ irreps are those labelled by $m=\ell+2 n$ with $n \in \mathbb{N}$. As a consequence, we find

$$
\left.\mathcal{D}_{S p(2, \mathbb{R})}(\ell+1)\right|_{U(1)}=\bigoplus_{n=0}^{\infty}[\ell+1+2 n]_{U(1)},
$$

which is indeed the correct decomposition.

We will see in section 5 that an important subgroup of $G$ to consider in the context of seesaw pairs is the maximal compact subgroup $K \subset G$. Indeed, one can show that the centralizer of $K$ in $S p(2 N, \mathbb{R})$, that we will denote $M^{\prime}$, contains $G^{\prime}$ so that $\left(K, M^{\prime}\right)$ is a dual pair and it forms a seesaw pair with $\left(G, G^{\prime}\right)$. The same property is true if we reverse $G$ and $G^{\prime}$ : the centralizer $M$ of a maximal compact subgroup $K^{\prime} \subset G^{\prime}$ also contains $G$. As a consequence, $\left(G, G^{\prime}\right)$ and $\left(K^{\prime}, M\right)$ also form a seesaw pair. In general, the groups $M$ and $M^{\prime}$ are not compact, in which case one can also consider their maximal compact subgroup, denoted by $K_{M}$ and $K_{M^{\prime}}$, respectively. It turns out that also they form a dual pair. We therefore end up with four dual pairs. The situation is depicted as

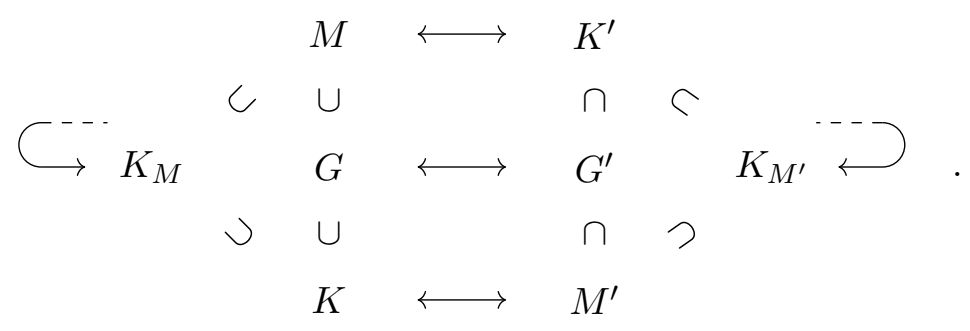

The application of the above diagram to each irreducible dual pair in table 1 is collected in appendix C.

Seesaw pairs are also useful for tensor products of the relevant representations and their plethysms. Say, we have a dual pair $\left(G, G^{\prime}\right) \subset S p(2 N, \mathbb{R})$ and their representations $\pi_{G}$ and $\pi_{G^{\prime}}$. The tensor product of the representation $\pi_{G}$ and its contragredient one $\bar{\pi}_{G}$ can be obtained by considering the seesaw pair,

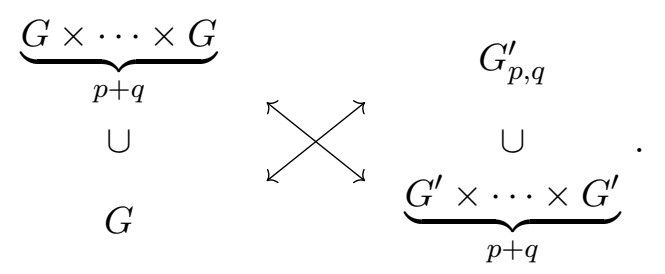

Here, the two pairs $\left(G, G_{p, q}^{\prime}\right)$ and $\left(G^{p+q}, G^{p+q}\right)$ are embedded in $S p(2(p+q) N, \mathbb{R})$. By examining the seesaw pairs for each irreducible pair $\left(G, G^{\prime}\right)$, one can find that the groups $G_{p, q}^{\prime}$ can be taken as in table 2. From the seesaw duality, we find that tensor product 


\begin{tabular}{|c|c|}
\hline$G^{\prime}$ & $G_{p, q}^{\prime}$ \\
\hline$G L(n, \mathbb{R})$ & $G L((p+q) n, \mathbb{R})$ \\
\hline$G L(n, \mathbb{C})$ & $G L((p+q) n, \mathbb{C})$ \\
\hline$U^{*}(2 n)$ & $U^{*}(2(p+q) n)$ \\
\hline$U(m, n)$ & $U(p m+q n, p n+q m)$ \\
\hline$O(m, n)$ & $O(p m+q n, p n+q m)$ \\
\hline$O(n, \mathbb{C})$ & $O((p+q) n, \mathbb{C})$ \\
\hline$O^{*}(2 n)$ & $O^{*}(2(p+q) n)$ \\
\hline$S p(2 n, \mathbb{R})$ & $S p(2(p+q) n, \mathbb{R})$ \\
\hline$S p(2 n, \mathbb{C})$ & $S p(2(p+q) n, \mathbb{C})$ \\
\hline$S p(m, n)$ & $S p(p m+q n, p n+q m)$ \\
\hline
\end{tabular}

Table 2. The list of $G_{p, q}^{\prime}$ for each irreducible $G^{\prime}$.

decompositions of $G$ representations can be obtained by the decomposition of $G_{p, q}^{\prime}$ representations:

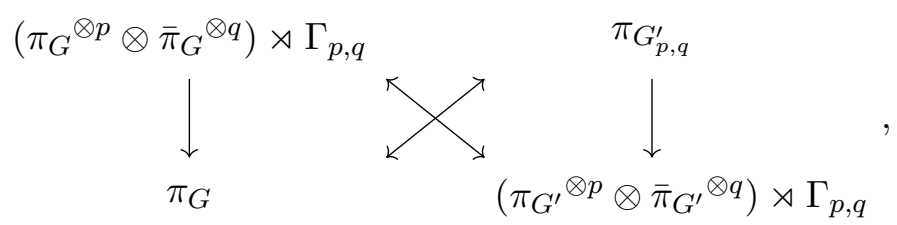

where the plethysm can be also controlled by a discrete group $\Gamma_{p, q}$ which is a subgroup of the symmetric group $\mathcal{S}_{p} \times \mathcal{S}_{q}$ permuting the same representations. In particular, every irreducible dual pair $\left(G, G^{\prime}\right)$, wherein $G^{\prime}$ is not the smallest group dual to $G$, can be used for the tensor product of the irreps of $G$ dual to the smallest $G^{\prime}$.

\section{Dual pairs of type $\left(G L_{M}, G L_{N}\right)$}

Let us describe the dual pair $\left(G L_{M}, G L_{N}\right) \subset S p(2 M N, \mathbb{R})$ in detail. Since it is embedded in $S p(2 M N, \mathbb{R})$, we introduce $M N$ pairs of conjugate operators

$$
\left(\omega_{A}^{I}, \tilde{\omega}_{I}^{A}\right), \quad A=1, \ldots, M, \quad I=1, \ldots, N,
$$

which obey the canonical commutation relations

$$
\left[\omega_{A}^{I}, \tilde{\omega}_{J}^{B}\right]=\delta_{A}^{B} \delta_{J}^{I}, \quad\left[\omega_{A}^{I}, \omega_{B}^{J}\right]=0=\left[\tilde{\omega}_{I}^{A}, \tilde{\omega}_{J}^{B}\right] .
$$

The $G L_{M}$ and $G L_{N}$ groups are generated respectively by

$$
\begin{gathered}
X_{B}^{A}=U_{\mathcal{W}}\left(\mathrm{X}^{A}{ }_{B}\right)=\frac{1}{2}\left\{\tilde{\omega}_{I}^{A}, \omega_{B}^{I}\right\}=\tilde{\omega}_{I}^{A} \omega_{B}^{I}+\frac{N}{2} \delta_{B}^{A}, \\
R_{I}^{J}=U_{\mathcal{W}}\left(\mathrm{R}_{I}^{J}\right)=\frac{1}{2}\left\{\tilde{\omega}_{I}^{A}, \omega_{A}^{J}\right\}=\tilde{\omega}_{I}^{A} \omega_{A}^{J}+\frac{M}{2} \delta_{I}^{J},
\end{gathered}
$$

where $U_{\mathcal{W}}$ is the metaplectic representation, and $\mathrm{X}^{A}{ }_{B}$ and $\mathrm{R}_{I}{ }^{J}$ are the matrices with components $\left(\mathrm{X}^{A}{ }_{B}\right)_{C}^{D}=\delta_{C}^{A} \delta_{B}^{D}$ and $\left(\mathrm{R}_{I}^{J}\right)^{K}{ }_{L}=\delta_{I}^{K} \delta_{L}^{J}$ generating $G L_{M}$ and $G L_{N}$ respectively. 
Notice that when $G L_{M}$ and $G L_{N}$ are realized as above, a specific ordering of $\omega$ and $\tilde{\omega}$ is chosen, namely the Weyl ordering. If we do not embed the pair $\left(G L_{M}, G L_{N}\right)$ in $S p(2 M N, \mathbb{R})$, one could take different orderings in each of the groups, leading to different factors of $\delta_{B}^{A}$ and $\delta_{I}^{J}$ (shift constants) in (3.3). Here, the embedding of the pair in $S p(2 M N, \mathbb{R})$ singles out a unique ordering, which fixes the shift constants, such that the diagonal $G L_{1}$ subgroups of the $G L_{M}$ and $G L_{N}$ coincide; i.e.

$$
Z:=X_{A}^{A}=R_{I}^{I}=\frac{1}{2}\left\{\omega_{A}^{I}, \tilde{\omega}_{I}^{A}\right\}=\tilde{\omega}_{I}^{A} \omega_{A}^{I}+\frac{M N}{2},
$$

which is a number operator with a constant shift.

Various real forms of $G L_{M}$ and $G L_{N}$ can be chosen by assigning different reality conditions to the operators $\omega_{A}^{I}$ and $\tilde{\omega}_{I}^{A}$. Such conditions can be straightforwardly deduced from the anti-involution $\sigma$ associated with each real form by asking that

$$
U_{\mathcal{W}}\left(\mathrm{X}^{A}{ }_{B}\right)^{\dagger}=U_{\mathcal{W}}\left(\sigma\left(\mathrm{X}^{A}{ }_{B}\right)\right), \quad U_{\mathcal{W}}\left(\mathrm{R}_{I}^{J}\right)^{\dagger}=U_{\mathcal{W}}\left(\sigma\left(\mathrm{R}_{I}^{J}\right)\right)
$$

See appendix B for the expressions of $\sigma$ for each real forms. In the following, we describe all such real forms by introducing their oscillator realizations.

\section{1 $(G L(M, \mathbb{R}), G L(N, \mathbb{R})) \subset S p(2 M N, \mathbb{R})$}

To single out the real forms $G L(M, \mathbb{R})$ and $G L(N, \mathbb{R})$, we impose the following reality conditions on $X^{A}{ }_{B}$ and $R_{I}{ }^{J}$

$$
\left(X_{B}^{A}\right)^{\dagger}=-X_{B}^{A}, \quad\left(R_{I}^{J}\right)^{\dagger}=-R_{I}^{J},
$$

which can be realized by requiring the $\omega$-operators to obey the reality conditions,

$$
\left(\omega_{A}^{I}\right)^{\dagger}=\omega_{A}^{I}, \quad\left(\tilde{\omega}_{I}^{A}\right)^{\dagger}=-\tilde{\omega}_{I}^{A} .
$$

Let us define

$$
a_{A}^{I}:=\frac{1}{\sqrt{2}}\left(\omega_{A}^{I}-\tilde{\omega}_{I}^{A}\right), \quad \tilde{a}_{I}^{A}:=\frac{1}{\sqrt{2}}\left(\tilde{\omega}_{I}^{A}+\omega_{A}^{I}\right)
$$

so that

$$
\left(a_{A}^{I}\right)^{\dagger}=\tilde{a}_{I}^{A}, \quad \text { and } \quad\left[a_{A}^{I}, \tilde{a}_{J}^{B}\right]=\delta_{A}^{B} \delta_{J}^{I},
$$

In terms of these creation and annihilation operators, the generators of $G L(M, \mathbb{R})$ and $G L(N, \mathbb{R})$ now read

$$
X_{B}^{A}=\frac{1}{2}\left(\tilde{a}_{I}^{A} a_{B}^{I}-\tilde{a}_{I}^{B} a_{A}^{I}+\tilde{a}_{I}^{A} \tilde{a}_{I}^{B}-a_{A}^{I} a_{B}^{I}\right)
$$

and

$$
R_{I}^{J}=\frac{1}{2}\left(\tilde{a}_{I}^{A} a_{A}^{J}-\tilde{a}_{J}^{A} a_{A}^{I}+\tilde{a}_{I}^{A} \tilde{a}_{J}^{A}-a_{A}^{I} a_{A}^{J}\right),
$$

and the common Abelian subgroup $G L(1, \mathbb{R}) \cong \mathbb{R}^{\times}$is generated by

$$
Z=\frac{1}{2}\left(\tilde{a}_{I}^{A} \tilde{a}_{I}^{A}-a_{A}^{I} a_{A}^{I}\right)
$$


Note that in the above expressions, the repeated indices are summed over even when they are positioned both up or both down. Since $G L(M, \mathbb{R})$ and $G L(N, \mathbb{R})$ are disconnected, ${ }^{10}$ we need also to take into account the reflection group elements $\mathcal{R}_{A}$ and $\mathcal{R}^{I}$,

$$
\mathcal{R}_{A}=\prod_{I=1}^{N} \mathcal{R}_{A}^{I}, \quad \mathcal{R}^{I}=\prod_{A=1}^{M} \mathcal{R}_{A}^{I},
$$

where $\mathcal{R}_{A}^{I}$ are the reflections in $S p(2 M N, \mathbb{R})$ introduced in section 2.1,

$$
\mathcal{R}_{A}^{I}\left(\begin{array}{c}
a_{B}^{J} \\
\tilde{a}_{J}^{B}
\end{array}\right)\left(\mathcal{R}_{A}^{I}\right)^{-1}=(-1)^{\delta_{A B} \delta^{I J}}\left(\begin{array}{c}
a_{B}^{J} \\
\tilde{a}_{J}^{B}
\end{array}\right) .
$$

Let us remark also that the elements,

$$
M_{A B}:=2 \delta_{C[A} X_{B]}^{C}=\tilde{a}_{I}^{A} a_{B}^{I}-\tilde{a}_{I}^{B} a_{A}^{I}, \quad N_{I J}:=2 R_{[I}^{K} \delta_{J] K}=\tilde{a}_{I}^{A} a_{A}^{J}-\tilde{a}_{J}^{A} a_{A}^{I},
$$

generate the maximal compact subgroups $O(M) \subset G L(M, \mathbb{R})$ and $O(N) \subset G L(N, \mathbb{R})$ respectively. Note that the generators of these compact groups take the form (creation) $\times$ (annihilation), so that they preserves the total oscillator number.

$3.2(G L(M, \mathbb{C}), G L(N, \mathbb{C})) \subset S p(4 M N, \mathbb{R})$

The real Lie groups $G L(M, \mathbb{C})$ and $G L(N, \mathbb{C})$ are obtained by starting with complex operators $\omega$ and $\tilde{\omega}$ with complex conjugates $\omega^{*}$ and $\tilde{\omega}^{*}$, obeying the commutation relations,

$$
\left[\omega_{A}^{I *}, \tilde{\omega}_{J}^{B *}\right]=\delta_{A}^{B} \delta_{J}^{I}
$$

and

$$
\left[\omega_{A}^{I}, \omega_{B}^{J *}\right]=\left[\omega_{A}^{I}, \tilde{\omega}_{J}^{B *}\right]=\left[\tilde{\omega}_{I}^{A}, \omega_{B}^{J *}\right]=\left[\tilde{\omega}_{I}^{A}, \tilde{\omega}_{J}^{B *}\right]=0 .
$$

We require the reality conditions,

$$
\left(\omega_{A}^{I}\right)^{\dagger}=\omega_{A}^{I *}, \quad\left(\tilde{\omega}_{I}^{A}\right)^{\dagger}=-\tilde{\omega}_{I}^{A *}, \quad\left(\omega_{A}^{I *}\right)^{\dagger}=\omega_{A}^{I}, \quad\left(\tilde{\omega}_{I}^{A *}\right)^{\dagger}=-\tilde{\omega}_{I}^{A}
$$

so that the generators,

$$
X_{ \pm B}^{A}=X_{B}^{A} \pm\left(X_{B}^{A}\right)^{*}, \quad R_{ \pm I}^{J}=R_{I}^{J} \pm\left(R_{I}^{J}\right)^{*}
$$

satisfy

$$
\left(X_{ \pm B}^{A}\right)^{\dagger}=\mp X_{ \pm B}^{A}, \quad\left(R_{ \pm I}^{J}\right)^{\dagger}=\mp R_{ \pm I}^{J} .
$$

Notice that $X_{+B}^{A}$ and $R_{+I}^{J}$ generate the $G L(M, \mathbb{R})$ and $G L(N, \mathbb{R})$ subgroup of $G L(M, \mathbb{C})$ and $G L(N, \mathbb{C})$, respectively. For the oscillator realization, we introduce

$$
\begin{array}{rlrl}
a_{A}^{I} & :=\frac{1}{\sqrt{2}}\left(\omega_{A}^{I}-\tilde{\omega}_{I}^{A *}\right), & \tilde{a}_{I}^{A}:=\frac{1}{\sqrt{2}}\left(\tilde{\omega}_{I}^{A}+\omega_{A}^{I *}\right), \\
b_{I}^{A}:=\frac{1}{\sqrt{2}}\left(\omega_{A}^{I *}-\tilde{\omega}_{I}^{A}\right), & \tilde{b}_{A}^{I}:=\frac{1}{\sqrt{2}}\left(\tilde{\omega}_{I}^{A *}+\omega_{A}^{I}\right),
\end{array}
$$

\footnotetext{
${ }^{10}$ The group $G L(N, \mathbb{R})$ is isomorphic to $\mathbb{R}^{\times} \ltimes S L(N, \mathbb{R})$, where $\mathbb{R}^{\times}$is the multiplicative group of real numbers. Since $\mathbb{R}^{\times}$has two connected components, positive and negative real numbers, $G L(N, \mathbb{R})$ has two connected components, whose elements have positive and negative determinant, respectively.
} 
so that

$$
\left(a_{A}^{I}\right)^{\dagger}=\tilde{a}_{I}^{A}, \quad\left(b_{I}^{A}\right)^{\dagger}=\tilde{b}_{A}^{I},
$$

and the only non-zero commutators are

$$
\left[a_{A}^{I}, \tilde{a}_{J}^{B}\right]=\delta_{A}^{B} \delta_{J}^{I}, \quad\left[b_{I}^{A}, \tilde{b}_{B}^{J}\right]=\delta_{B}^{A} \delta_{I}^{J} .
$$

Note that under complex conjugation, the $a$ and $b$ oscillators are mapped to one another:

$$
\left(a_{A}^{I}\right)^{*}=b_{I}^{A}, \quad\left(\tilde{a}_{I}^{A}\right)^{*}=\tilde{b}_{A}^{I} .
$$

In terms of these oscillators, the generators $X^{A}{ }_{B}$ and $R_{I}{ }^{J}$ read

$$
X_{B}^{A}=\frac{1}{2}\left(\tilde{a}_{I}^{A} a_{B}^{I}-\tilde{b}_{B}^{I} b_{I}^{A}+\tilde{a}_{I}^{A} \tilde{b}_{B}^{I}-b_{I}^{A} a_{B}^{I}\right),
$$

and

$$
R_{I}^{J}=\frac{1}{2}\left(\tilde{a}_{I}^{A} a_{A}^{J}-\tilde{b}_{A}^{J} b_{I}^{A}+\tilde{a}_{I}^{A} \tilde{b}_{A}^{J}-b_{I}^{A} a_{A}^{J}\right) .
$$

From the above, one can easily find the generators $X_{ \pm B}^{A}$ and $R_{ \pm I}^{J}$ using (3.19) and (3.24). The common Abelian subgroup $G L(1, \mathbb{C}) \cong \mathbb{C}^{\times} \cong \mathbb{R}^{+} \times U(1)$ is generated by

$$
Z_{+}=\tilde{a}_{I}^{A} \tilde{b}_{A}^{I}-a_{A}^{I} b_{I}^{A}, \quad \text { and } \quad Z_{-}=\tilde{a}_{I}^{A} a_{A}^{I}-\tilde{b}_{A}^{I} b_{I}^{A},
$$

which can be interpreted as the radial and angular part of the complex plane without origin $\mathbb{C}^{\times}$, respectively. The maximal compact subgroups $U(M)$ and $U(N)$ of $G L(M, \mathbb{C})$ and $G L(N, \mathbb{C})$ are generated respectively by $X^{A}{ }_{B}-\left(X^{B}{ }_{A}\right)^{*}$ and $R_{I}^{J}-\left(R_{J}^{I}\right)^{*}$, and they have the form (creation) $\times\left(\right.$ annihilation). Their common $U(1)$ generator coincides with $Z_{-}$.

\section{$3.3 \quad\left(U^{*}(2 M), U^{*}(2 N)\right) \subset S p(8 M N, \mathbb{R})$}

To single out the real forms $U^{*}(2 M)$ and $U^{*}(2 N),{ }^{11}$ we require $X^{A}{ }_{B}$ and $R_{I}{ }^{J}$ to satisfy

$$
\left(X_{B}^{A}\right)^{\dagger}=-\Omega_{A C} X^{C}{ }_{D} \Omega^{D B}, \quad\left(R_{I}^{J}\right)^{\dagger}=-\Omega^{I K} R_{K}{ }^{L} \Omega_{L J} .
$$

This can be realized by requiring the $\omega$ operators to obey the reality conditions

$$
\left(\omega_{A}^{I}\right)^{\dagger}=\Omega_{I J} \Omega^{A B} \omega_{B}^{J}, \quad\left(\tilde{\omega}_{I}^{A}\right)^{\dagger}=-\Omega^{I J} \Omega_{A B} \tilde{\omega}_{J}^{B},
$$

where the $A, B$, and $I, J$ indices take respectively $2 M$ and $2 N$ values. The two matrices $\Omega_{A B}$ and $\Omega^{I J}$ are both antisymmetric and square to minus the identity matrix, and $\Omega^{A B}$ and $\Omega_{I J}$ are their respective inverses: $\Omega^{A B} \Omega_{B C}=\delta_{C}^{A}, \Omega_{I J} \Omega^{J K}=\delta_{I}^{K}$. As a consequence, $\Omega_{A B}=-\Omega^{A B}$ and $\Omega_{I J}=-\Omega^{I J}$. Upon defining the oscillators,

$$
a_{A}^{I}:=\frac{1}{\sqrt{2}}\left(\omega_{A}^{I}-\Omega_{A B} \Omega^{I J} \tilde{\omega}_{J}^{B}\right), \quad \tilde{a}_{I}^{A}:=\left(a_{A}^{I}\right)^{\dagger},
$$

with

$$
\left[a_{A}^{I}, \tilde{a}_{J}^{B}\right]=\delta_{A}^{B} \delta_{J}^{I} .
$$

\footnotetext{
${ }^{11}$ Notice that the determinant of an element of $U^{*}(2 N)$ is always positive, and hence the group $U^{*}(2 N)$ is isomorphic to $\mathbb{R}^{+} \times S U^{*}(2 N)$.
} 
the generators $X^{A}{ }_{B}$ and $R_{I}^{J}$ can be expressed as

$$
\begin{aligned}
X_{B}^{A} & =\frac{1}{2}\left(\tilde{a}_{I}^{A} a_{B}^{I}-\tilde{a}_{B}^{I} a_{I}^{A}-a_{I}^{A} a_{B}^{I}+\tilde{a}_{I}^{A} \tilde{a}_{B}^{I}\right), \\
R_{I}{ }^{J} & =\frac{1}{2}\left(\tilde{a}_{I}^{A} a_{A}^{J}-\tilde{a}_{A}^{J} a_{I}^{A}-a_{I}^{A} a_{A}^{J}+\tilde{a}_{I}^{A} \tilde{a}_{A}^{J}\right),
\end{aligned}
$$

where we used $\Omega_{A B}$ and $\Omega_{I J}$ (and their inverse) to raise and lower indices as in (1.11). The common center $\mathbb{R}^{+}$of $U^{*}(2 M)$ and $U^{*}(2 N)$ is generated by

$$
Z=\tilde{a}_{I}^{A} \tilde{a}_{A}^{I}-a_{A}^{I} a_{I}^{A}
$$

The Lie algebras of the maximal compact subgroups $S p(M) \subset U^{*}(2 M)$ and $S p(N) \subset$ $U^{*}(2 N)$ are generated respectively by

$$
X^{(A B)}=\tilde{a}_{I}^{(A} a^{B) I}, \quad R_{(I J)}=\tilde{a}_{(I}^{A} a_{J) A} .
$$

Again, one can see that the generators of these compact subgroups have the form of (creation $) \times($ annihilation $)$.

$3.4\left(U\left(M_{+}, M_{-}\right), U\left(N_{+}, N_{-}\right)\right) \subset S p\left(2\left(M_{+}+M_{-}\right)\left(N_{+}+N_{-}\right), \mathbb{R}\right)$

To single out the real forms $U\left(M_{+}, M_{-}\right)$and $U\left(N_{+}, N_{-}\right)$, we impose

$$
\left(X_{B}^{A}\right)^{\dagger}=\eta^{A D} \eta_{B C} X^{C}{ }_{D}, \quad\left(R_{I}^{J}\right)^{\dagger}=\eta_{I L} \eta^{J K} R_{K}{ }^{L},
$$

where $\eta_{A B}$ and $\eta^{I J}$ are diagonal matrices of signature $\left(M_{+}, M_{-}\right)$and $\left(N_{+}, N_{-}\right)$respectively, and where $X^{A}{ }_{B}$ and $R_{I}^{J}$ are defined in (3.3). This can be achieved by requiring the $\omega$ operators to obey the reality condition,

$$
\left(\omega_{A}^{I}\right)^{\dagger}=\eta_{A B} \eta^{I J} \tilde{\omega}_{J}^{B}
$$

To introduce the oscillator realization, we split the $A$ and $I$ indices into $(a$, a) and $(i, \mathrm{i})$ and set $\eta_{A B}=\left(\delta_{a b},-\delta_{\mathrm{ab}}\right)$ and $\eta^{I J}=\left(\delta^{i j},-\delta^{\mathrm{ij}}\right)$. Here, $a$ and a take respectively $M_{+}$and $M_{-}$values, whereas $i$ and $i$ take respectively $N_{+}$and $N_{-}$values. Upon defining

$$
a_{a}^{i}:=\omega_{a}^{i}, \quad b_{i}^{\mathrm{a}}:=\tilde{\omega}_{i}^{\mathrm{a}}, \quad c_{\mathrm{i}}^{a}:=\tilde{\omega}_{\mathrm{i}}^{a}, \quad d_{\mathrm{a}}^{\mathrm{i}}:=\omega_{\mathrm{a}}^{\mathrm{i}},
$$

and

$$
\tilde{a}_{i}^{a}=\left(a_{a}^{i}\right)^{\dagger}, \quad \tilde{b}_{\mathrm{a}}^{i}=\left(b_{i}^{\mathrm{a}}\right)^{\dagger}, \quad \tilde{c}_{a}^{\mathrm{i}}=\left(c_{\mathrm{i}}^{a}\right)^{\dagger}, \quad \tilde{d}_{\mathrm{i}}^{\mathrm{a}}=\left(d_{\mathrm{a}}^{\mathrm{i}}\right)^{\dagger},
$$

we end up with $\left(M_{+}+M_{-}\right)\left(N_{+}+N_{-}\right)$canonical pairs of creation and annihilation operators, as these oscillators obey

$$
\left[a_{a}^{i}, \tilde{a}_{j}^{b}\right]=\delta_{j}^{i} \delta_{a}^{b}, \quad\left[b_{j}^{\mathrm{a}}, \tilde{b}_{\mathrm{b}}^{i}\right]=\delta_{j}^{i} \delta_{\mathrm{b}}^{\mathrm{a}}, \quad\left[c_{\mathrm{i}}^{a}, \tilde{c}_{b}^{\mathrm{j}}\right]=\delta_{b}^{a} \delta_{\mathrm{i}}^{\mathrm{j}}, \quad\left[d_{\mathrm{a}}^{\mathrm{i}}, \tilde{d}_{\mathrm{j}}^{\mathrm{b}}\right]=\delta_{\mathrm{a}}^{\mathrm{b}} \delta_{\mathrm{j}}^{\mathrm{i}},
$$


with all other commutators vanishing. In terms of the $a, b, c$ and $d$ oscillators, the generators $X^{A}{ }_{B}$ and $R_{I}^{J}$ read

$$
\begin{array}{ll}
X_{b}^{a}=\tilde{a}_{i}^{a} a_{b}^{i}-\tilde{c}_{b}^{\mathrm{i}} c_{\mathrm{i}}^{a}+\frac{N_{+}-N_{-}}{2} \delta_{b}^{a}, & X^{\mathrm{a}}{ }_{b}=a_{b}^{i} b_{i}^{\mathrm{a}}-\tilde{c}_{b}^{\mathrm{i}} \tilde{d}_{\mathrm{i}}^{\mathrm{a}}, \\
X^{a}{ }_{\mathrm{b}}=-\tilde{a}_{i}^{a} \tilde{b}_{\mathrm{b}}^{i}+c_{\mathrm{i}}^{a} d_{\mathrm{b}}^{\mathrm{i}}, & X^{\mathrm{a}}{ }_{\mathrm{b}}=-\tilde{b}_{\mathrm{b}}^{i} b_{i}^{\mathrm{a}}+\tilde{d}_{\mathrm{i}}^{\mathrm{a}} d_{\mathrm{b}}^{\mathrm{i}}-\frac{N_{+}-N_{-}}{2} \delta_{\mathrm{b}}^{\mathrm{a}}, \\
R_{j}{ }^{i}=\tilde{a}_{j}^{a} a_{a}^{i}-\tilde{b}_{\mathrm{a}}^{i} b_{j}^{\mathrm{a}}+\frac{M_{+}-M_{-}}{2} \delta_{j}^{i}, & R_{j}{ }^{\mathrm{i}}=-\tilde{a}_{j}^{a} \tilde{c}_{a}^{\mathrm{i}}+b_{j}^{\mathrm{a}} d_{\mathrm{a}}^{\mathrm{i}}, \\
R_{\mathrm{j}}{ }^{i}=a_{a}^{i} c_{\mathrm{j}}^{a}-\tilde{b}_{\mathrm{a}}^{i} \tilde{d}_{\mathrm{j}}^{\mathrm{a}}, & R_{\mathrm{j}}{ }^{\mathrm{i}}=-\tilde{c}_{a}^{\mathrm{i}} c_{\mathrm{j}}^{a}+\tilde{d}_{\mathrm{j}}^{\mathrm{a}} d_{\mathrm{a}}^{\mathrm{i}}-\frac{M_{+}-M_{-}}{2} \delta_{\mathrm{j}}^{\mathrm{i}},
\end{array}
$$

and the common $U(1)$ center of $U\left(M_{+}, M_{-}\right)$and $U\left(N_{+}, N_{-}\right)$is generated by ${ }^{12}$

$$
Z=\tilde{a}_{i}^{a} a_{a}^{i}-\tilde{b}_{\mathrm{a}}^{i} b_{i}^{\mathrm{a}}-\tilde{c}_{a}^{\mathrm{i}} c_{\mathrm{i}}^{a}+\tilde{d}_{\mathrm{i}}^{\mathrm{a}} d_{\mathrm{a}}^{\mathrm{i}}+\frac{1}{2}\left(N_{+}-N_{-}\right)\left(M_{+}-M_{-}\right) .
$$

The maximal compact subgroups $U\left(M_{+}\right) \times U\left(M_{-}\right) \subset U\left(M_{+}, M_{-}\right)$and $U\left(N_{+}\right) \times U\left(N_{-}\right) \subset$ $U\left(N_{+}, N_{-}\right)$are respectively generated by $X^{a}{ }_{b}$ and $X^{\mathrm{a}}{ }_{\mathrm{b}}$, and $R_{j}{ }^{i}$ and $R_{\mathrm{j}}{ }^{\mathrm{i}}$. Note also that when $N_{-}=0$ or $M_{-}=0$, that is, when one of the group, say $G$, in the pair $\left(G, G^{\prime}\right)$ is compact, the Lie algebra of the dual group $G^{\prime}$ can be decomposed into eigenspaces of the total number operator with eigenvalues $+2,0,-2$. In other words, they do not have the mixed form of $(\text { creation })^{2}+(\text { annihilation })^{2}$. This property will prove useful when decomposing the Fock space into irreducible representations.

\section{Dual pairs of type $\left(O_{N}, S p_{2 M}\right)$}

To describe the dual pairs $\left(O_{N}, S p_{2 M}\right) \subset S p(2 N M, \mathbb{R})$ in detail, we consider again $M N$ pairs of operators. In this case, it is more convenient to use $2 M N$ operators without explicitly pairing them, which we denote by

$$
y_{A}^{I}, \quad A=1, \ldots, N, \quad I=1, \ldots, 2 M,
$$

and which satisfy

$$
\left[y_{A}^{I}, y_{B}^{J}\right]=E_{A B} \Omega^{I J},
$$

where $E_{A B}$ and $\Omega^{I J}$ are symmetric and antisymmetric invertible matrices of dimension $N$ and $2 M$, respectively. Then, the reductive subgroups $O_{N}$ and $S p_{2 M}$ are generated respectively by

$$
M_{A B}=U_{\mathcal{W}}\left(\mathrm{M}_{A B}\right)=\Omega_{I J} y_{[A}^{I} y_{B]}^{J}, \quad K^{I J}=U_{\mathcal{W}}\left(\mathrm{K}^{I J}\right)=E^{A B} y_{A}^{(I} y_{B}^{J)},
$$

where $U_{\mathcal{W}}$ is the metaplectic representation, and $\mathrm{M}_{A B}$ and $\mathrm{K}^{I J}$ are the matrices with components $\left(\mathrm{M}_{A B}\right)^{C D}=2 \delta_{[A}^{C} \delta_{B]}^{D}$ and $\left(\mathrm{K}^{I J}\right)_{K L}=2 \delta_{K}^{(I} \delta_{L}^{J)}$ generating $O_{N}$ and $S p_{2 M}$. The commutation relations of $M_{A B}$ are

$$
\left[M_{A B}, M_{C D}\right]=2\left(E_{D[A} M_{B] C}-E_{C[A} M_{B] D}\right),
$$

\footnotetext{
${ }^{12}$ Notice that the operators in (3.41) and (3.42) do not have integer eigenvalues when $N_{+}-N_{-}$and $M_{+}-M_{-}$are both odd. In this case, the realizations of $U\left(N_{+}, N_{-}\right)$and $U\left(M_{+}, M_{-}\right)$are double-valued.
} 
whereas those of $K^{I J}$ are

$$
\left[K^{I J}, K^{K L}\right]=2\left(\Omega^{I(K} K^{L) J}+\Omega^{J(K} K^{L) I}\right) .
$$

Note here that we have not specified the signature of the flat metric $E_{A B}$. Clearly, at the level of the complex Lie algebra, different $E_{A B}$ can be all brought to the form $\delta_{A B}$ by suitably redefining the $y$-operators. We leave the ambiguity of $E_{A B}$ at this stage since there is a preferable choice of $E_{A B}$ in each real form of $O_{N}$ to obtain a compact expression of their generators.

Various real forms of $O_{N}$ and $S p_{2 M}$ can be chosen by assigning different reality conditions to the operators $y_{A}^{I}$. Such conditions can be straightforwardly deduced from the anti-involution $\tilde{\sigma}$ associated with each real form by asking

$$
U_{\mathcal{W}}\left(\mathrm{M}_{A B}\right)^{\dagger}=U_{\mathcal{W}}\left(\tilde{\sigma}\left(\mathrm{M}_{A B}\right)\right), \quad U_{\mathcal{W}}\left(\mathrm{K}^{I J}\right)^{\dagger}=U_{\mathcal{W}}\left(\tilde{\sigma}\left(\mathrm{K}^{I J}\right)\right) .
$$

See appendix B for the expression of $\tilde{\sigma}$ in each real forms. In the following, we describe all such real forms by introducing their oscillator realizations.

\section{$4.1\left(O\left(N_{+}, N_{-}\right), S p(2 M, \mathbb{R})\right) \subset S p\left(2 M\left(N_{+}+N_{-}\right), \mathbb{R}\right)$}

To identify the real forms $O\left(N_{+}, N_{-}\right)$and $S p(2 M, \mathbb{R})$, we set $E_{A B}=\eta_{A B}$, the flat metric of signature $\left(N_{+}, N_{-}\right)$, and require that the generators $M_{A B}$ and $K^{I J}$ satisfy the reality conditions,

$$
\left(M_{A B}\right)^{\dagger}=-M_{A B}, \quad\left(K^{I J}\right)^{\dagger}=J_{I K} K^{K L} J_{L J},
$$

where the matrix $J_{I J}$ satisfy $J_{I J}=J_{J I}, J_{I J} J_{J K}=\delta_{I K}$ and $J_{I J} \Omega_{J K}=-\Omega_{I J} J_{J K} \cdot{ }^{13}$ The above can be realized by requiring the $y_{A}^{I}$ operators to obey the reality condition,

$$
\left(y_{A}^{I}\right)^{\dagger}=J_{I J} y_{A}^{J} .
$$

In order to introduce the oscillator realization, we split the $A$ and $I$ indices into $A=(a, \mathrm{a})$ and $I=(+i,-i)$ where $a$ and a take respectively $N_{+}$and $N_{-}$values. In this convention, $\eta_{A B}=\left(\delta_{a b},-\delta_{\mathrm{ab}}\right), \Omega^{+i-j}=\delta^{i j}$ and $J_{+i-j}=\delta_{i j}$, and the condition (4.10) becomes

$$
\left(y_{A}^{ \pm i}\right)^{\dagger}=y_{A}^{\mp i} .
$$

We therefore introduce

$$
a_{a}^{i}=y_{a}^{+i}, \quad \tilde{a}_{i}^{a}=y_{a}^{-i}, \quad b_{i}^{\mathrm{a}}=y_{\mathrm{a}}^{-i}, \quad \tilde{b}_{i}^{\mathrm{a}}=y_{\mathrm{a}}^{+i},
$$

${ }^{13}$ Note here that we could use the more familiar reality condition,

$$
\left(K^{I J}\right)^{\dagger}=-K^{I J}
$$

which can be realized by the operators satisfying

$$
\left(y_{A}^{I}\right)^{\dagger}=i y_{A}^{I} .
$$

Even though the above seems simpler, it actually leads to slightly more involved and unfamiliar expression for $K^{I J}$ in terms of oscillators. That is why we chose another, but equivalent, reality condition for $K^{I J}$. 
which obey

$$
\tilde{a}_{i}^{a}=\left(a_{a}^{i}\right)^{\dagger}, \quad \tilde{b}_{\mathrm{a}}^{i}=\left(b_{i}^{\mathrm{a}}\right)^{\dagger},
$$

and the canonical commutation relation

$$
\left[a_{a}^{i}, \tilde{a}_{j}^{b}\right]=\delta_{j}^{i} \delta_{a}^{b}, \quad\left[b_{i}^{\mathrm{a}}, \tilde{b}_{\mathrm{b}}^{j}\right]=\delta_{i}^{j} \delta_{\mathrm{b}}^{\mathrm{a}} .
$$

In terms of the canonical pairs $a$ and $b$, the generators $M_{A B}$ and $K^{I J}$ read

$$
M_{a b}=\tilde{a}_{i}^{a} a_{b}^{i}-\tilde{a}_{i}^{b} a_{a}^{i}, \quad M_{a \mathrm{~b}}=\tilde{a}_{i}^{a} \tilde{b}_{\mathrm{b}}^{i}-a_{a}^{i} b_{i}^{\mathrm{b}}, \quad M_{\mathrm{ab}}=\tilde{b}_{\mathrm{b}}^{i} b_{i}^{\mathrm{a}}-\tilde{b}_{\mathrm{a}}^{i} b_{i}^{\mathrm{b}},
$$

and

$$
\begin{array}{ll}
K^{+i+j}=a_{a}^{i} a_{a}^{j}-\tilde{b}_{\mathrm{a}}^{i} \tilde{b}_{\mathrm{a}}^{j}, & K^{-i-j}=\tilde{a}_{i}^{a} \tilde{a}_{j}^{a}-b_{i}^{\mathrm{a}} b_{j}^{\mathrm{a}}, \\
K^{+i-j} & =\tilde{a}_{j}^{a} a_{a}^{i}-\tilde{b}_{\mathrm{a}}^{i} b_{j}^{\mathrm{a}}+\frac{N_{+}-N_{-}}{2} \delta_{j}^{i} .
\end{array}
$$

Since $O\left(N_{+}, N_{-}\right)$has 4 connected components (2 components when $N_{+}$or $N_{-}$vanishes), we need to take into account the reflections,

$$
\mathcal{R}_{a}=\prod_{i=1}^{M} \mathcal{R}_{a}^{i}, \quad \mathcal{R}_{\mathrm{a}}=\prod_{i=1}^{M} \mathcal{R}_{\mathrm{a}}^{i},
$$

where $\mathcal{R}_{a}^{i}$ and $\mathcal{R}_{\mathrm{a}}^{i}$ flip the sign of $a_{a}^{i}$ and $b_{\mathrm{a}}^{i}$ respectively.

Notice that $M_{a b}$ and $M_{\mathrm{ab}}$ generate the maximal compact subgroup $O\left(N_{+}\right) \times$ $O\left(N_{-}\right)$of $O\left(N_{+}, N_{-}\right)$, while $K^{+i-j}$ generate the maximal compact subgroup $U(M)$ of $\operatorname{Sp}(2 M, \mathbb{R})$. When $N_{-}=0$, no generator of $S p(2 M, \mathbb{R})$ has the mixed form of $(\text { creation })^{2}+(\text { annihilation })^{2}$. Again, this will prove useful in decomposing the Fock space into irreducible representations.

$4.2(O(N, \mathbb{C}), S p(2 M, \mathbb{C})) \subset S p(4 N M, \mathbb{R})$

The real Lie groups $O(N, \mathbb{C})$ and $S p(2 M, \mathbb{C})$, are obtained by starting with complex operators $y$ with complex conjugates $y^{*}$, obeying the commutation relations,

$$
\left[y_{A}^{I *}, y_{B}^{J *}\right]=\delta_{A B} \Omega^{I J}, \quad\left[y_{A}^{I}, y_{B}^{J *}\right]=0 .
$$

We require the reality condition,

$$
\left(y_{A}^{I}\right)^{\dagger}=J_{I J} y_{A}^{J *},
$$

so that

$$
\left(M_{A B}\right)^{\dagger}=-\left(M_{A B}\right)^{*}, \quad\left(K^{I J}\right)^{\dagger}=J_{I K}\left(K^{K L}\right)^{*} J_{L J},
$$

and hence the generators of $O(N, \mathbb{C})$, which are given by

$$
M_{A B}^{ \pm}:=M_{A B} \pm\left(M_{A B}\right)^{*}, \quad K_{ \pm}^{I J}:=K^{I J} \pm\left(K^{I J}\right)^{*},
$$

satisfy

$$
\left(M_{A B}^{ \pm}\right)^{\dagger}=\mp M_{A B}^{ \pm}, \quad\left(K_{ \pm}^{I J}\right)^{\dagger}= \pm J_{I K} K_{ \pm}^{I J} J_{L J},
$$


and $M_{A B}^{+}$and $K_{+}^{I J}$ generate the subgroups $O(N)$ and $S p(2 M, \mathbb{R})$ of $O(N, \mathbb{C})$ and $S p(2 M, \mathbb{C})$ respectively. To define the oscillator realization, we introduce

$$
a_{A}^{I}:=\frac{1}{\sqrt{2}}\left(y_{A}^{I}+\eta_{I J} y_{A}^{J *}\right), \quad \tilde{a}_{I}^{A}=\frac{1}{\sqrt{2}} \Omega_{I J}\left(-y_{A}^{J}+\eta_{J K} y_{A}^{K *}\right)
$$

where $\eta_{I J}=\left(\delta_{+i+j},-\delta_{-i-j}\right)$ and satisfies $J_{I K} \eta_{K J}=\Omega_{I J}$ so that

$$
\left(a_{A}^{I}\right)^{\dagger}=\tilde{a}_{I}^{A}, \quad\left(a_{A}^{I}\right)^{*}=\eta_{I J} a_{A}^{J}, \quad\left[a_{A}^{I}, \tilde{a}_{J}^{B}\right]=\delta_{A}^{B} \delta_{J}^{I} .
$$

In terms of these oscillators, the generators $M_{A B}$ and $K^{I J}$ read

$$
M_{A B}=\frac{1}{2}\left(\tilde{a}_{I}^{A} a_{B}^{I}-\tilde{a}_{I}^{B} a_{A}^{I}-\tilde{a}_{I}^{A} \tilde{a}^{B I}-a_{A I} a_{B}^{I}\right),
$$

and

$$
K^{I J}=\frac{1}{2}\left(a_{A}^{I} a_{A}^{J}+\tilde{a}^{A I} \tilde{a}^{A J}-2 \tilde{a}^{A(I} a_{A}^{J)}\right) .
$$

Since $O(N, \mathbb{C})$ has two connected components, corresponding to elements of determinant \pm 1 respectively, we need to take into account the reflections,

$$
\mathcal{R}_{A}=\prod_{I=1}^{2 M} \mathcal{R}_{A}^{I},
$$

where $\mathcal{R}_{A}^{I}$ flips the sign of $a_{A}^{I}$. The generators of the maximal compact subgroup $O(N) \subset$ $O(N, \mathbb{C}) \mathrm{read}$

$$
M_{A B}^{+}=\tilde{a}_{I}^{A} a_{B}^{I}-\tilde{a}_{I}^{B} a_{A}^{I},
$$

while the generators of $S p(M) \subset S p(2 M, \mathbb{C})$ are given by

$$
K^{I J}-\eta_{I K} \eta_{J L}\left(K^{K L}\right)^{*}=\Omega^{K(I} \tilde{a}_{K}^{A} a_{A}^{J)} .
$$

Once again, we can notice that the maximal compact subgroups $O(N)$ and $S p(M)$ are generated by operators of the form (creation) $\times$ (annihilation).

\section{$4.3\left(O^{*}(2 N), S p\left(M_{+}, M_{-}\right)\right) \subset S p\left(4 N\left(M_{+}+M_{-}\right), \mathbb{R}\right)$}

To identify the real forms $O^{*}(2 N)$ and $S p\left(M_{+}, M_{-}\right)$, we begin with the metric $E_{A B}=J_{A B}$ where $J_{A B}$ is the symmetric off-diagonal matrix defined, in terms of $A=(+a,-a)$, as

$$
J_{ \pm a \pm b}=0, \quad J_{ \pm a \mp b}=\delta_{a b} .
$$

The reality conditions to be imposed on $M_{A B}$ and $K^{I J}$ are

$$
\left(M_{A B}\right)^{\dagger}=-\Omega^{A C} M_{C D} \Omega^{D B}, \quad\left(K^{I J}\right)^{\dagger}=\Psi_{I K} K^{K L} \Psi_{L J},
$$

where $\Psi_{I J}$ is an antisymmetric matrix given by

$$
\Psi_{ \pm i \pm j}=0, \quad \Psi_{\mp i \pm j}= \pm \eta_{i j}
$$


with the flat metric $\eta_{i j}$ of signature $\left(M_{+}, M_{-}\right)$. Here, we used $I=(+i,-i)$ where $\Omega^{+i-j}=$ $\delta^{i j}$. The conditions (4.32) can be realized by requiring the $y$ operators to obey the reality condition,

$$
\left(y_{A}^{I}\right)^{\dagger}=\Omega^{A B} \Psi_{I J} y_{B}^{J} .
$$

Splitting again the $A$ and $i$ indices into $A=(+a,-a)$ and $i=(r, \mathrm{r})$ so that $\Omega^{+a-b}=\delta^{a b}$ and $\eta_{i j}=\left(\delta_{r s},-\delta_{\mathrm{rs}}\right)$, the reality condition reads

$$
\left(y_{+a}^{+r}\right)^{\dagger}=-y_{-a}^{-r}, \quad\left(y_{-a}^{+r}\right)^{\dagger}=y_{+a}^{-r}, \quad\left(y_{+a}^{+\mathbf{r}}\right)^{\dagger}=y_{-a}^{-\mathrm{r}}, \quad\left(y_{-a}^{+\mathrm{r}}\right)^{\dagger}=-y_{+a}^{-\mathrm{r}} .
$$

By repairing the symplectic indices as $R=(+r,-r)$ and $\mathrm{R}=(+\mathrm{r},-\mathrm{r})$, we introduce

$$
a_{R}^{a}=\Omega_{R S} y_{-a}^{S}, \quad \tilde{a}_{a}^{R}=y_{+a}^{R}, \quad b_{\mathrm{R}}^{a}=\Omega_{\mathrm{RS}} y_{+a}^{\mathrm{S}}, \quad \tilde{b}_{a}^{\mathrm{R}}=y_{-a}^{\mathrm{R}},
$$

where $\Omega_{-r+s}=\delta_{r s}$ and $\Omega_{-\mathrm{r}+\mathrm{s}}=\delta_{\mathrm{rs}}$. These $2 N\left(M_{+}+M_{-}\right)$pairs of oscillators satisfy

$$
\left[a_{R}^{a}, \tilde{a}_{b}^{S}\right]=\delta_{R}^{S} \delta_{b}^{a}, \quad\left[b_{\mathrm{R}}^{a}, \tilde{b}_{b}^{\mathrm{S}}\right]=\delta_{\mathrm{R}}^{\mathrm{S}} \delta_{b}^{a} \quad \tilde{a}_{a}^{R}=\left(a_{R}^{a}\right)^{\dagger}, \quad \tilde{b}_{a}^{\mathrm{R}}=\left(b_{\mathrm{R}}^{a}\right)^{\dagger} .
$$

In terms of $a, b$ oscillators, the generators $M_{A B}$ read

$$
\begin{array}{ll}
M_{+a-b}=\tilde{a}_{a}^{R} a_{R}^{b}-\tilde{b}_{b}^{\mathrm{R}} b_{\mathrm{R}}^{a}+\left(M_{+}-M_{-}\right) \delta_{a b}, & \\
M_{+a+b}=2 \tilde{a}_{[a}^{R} \tilde{a}_{b] R}+2 b^{\mathrm{R}[a} b_{\mathrm{R}}^{b]}, & M_{-a-b}=2 a^{R[a} a_{R}^{b]}+2 \tilde{b}_{[a}^{\mathrm{R}} \tilde{b}_{b] \mathrm{R}},
\end{array}
$$

and $K^{I J}$ has altogether 3 types of components given by

$$
K^{R S}=2 \tilde{a}_{a}^{(R} a^{S) a}, \quad K^{\mathrm{RS}}=2 \tilde{b}_{a}^{(\mathrm{R}} b^{\mathrm{S}) a}, \quad K^{R \mathrm{R}}=a^{R a} b^{\mathrm{S} a}+\tilde{a}_{a}^{R} \tilde{b}_{a}^{\mathrm{R}},
$$

where the $R, S$ and R,S indices are lowered and raised by $\Omega_{R S}, \Omega^{R S}$, and $\Omega_{\mathrm{RS}}, \Omega^{\mathrm{RS}}$ as in (1.11). Notice that $M_{+a-b}$ generate the maximal compact subgroup $U(N)$ of $O^{*}(2 N)$, while $K^{R S}$ and $K^{\mathrm{RS}}$ generate the maximal compact subgroup $S p\left(M_{+}\right) \times S p\left(M_{-}\right)$of $S p\left(M_{+}, M_{-}\right)$. When $M_{-}=0$, no generator of $O^{*}(2 N)$ is of the form (creation) ${ }^{2}+$ $(\text { annihilation })^{2}$.

\section{Representations of compact dual pairs}

In the previous sections, we have introduced oscillator realizations for various dual pairs, and made two observations:

1. Generators of compact (sub)groups $K \subseteq G$ of dual pairs $\left(G, G^{\prime}\right)$ are of the form (creation) $\times($ annihilation $)$. In other words, the Lie algebra of $K$ is the eigenspace of the total number operator with eigenvalue 0 .

2. When $K=G$, the dual group $G^{\prime}$ does not have any generators of the mixed form, $(\text { creation })^{2}+(\text { annihilation })^{2} .{ }^{14}$ In other words, the Lie algebra of $G^{\prime}$ can be decomposed into eigenspaces of the total number operator with eigenvalues $+2,0,-2$ :

$$
\mathfrak{g}^{\prime}=\mathfrak{g}_{+2}^{\prime} \oplus \mathfrak{g}_{0}^{\prime} \oplus \mathfrak{g}_{-2}^{\prime} .
$$

The subalgebra $\mathfrak{g}_{0}^{\prime}$ corresponds to the Lie algebra of the maximal compact subgroup $K^{\prime}$ of $G^{\prime}$.

\footnotetext{
${ }^{14}$ The only exception is the duality $\left(O^{*}(2) \cong U(1), S p\left(M_{+}, M_{-}\right)\right)$, detailed in section 6.1 .
} 
To analyze the representations of the dual pairs, we first construct the Fock space $\mathcal{W}$ using the oscillators in the usual manner:

$$
\mathcal{W}=\bigoplus_{n=0}^{\infty} W_{n}, \quad W_{n}=\operatorname{span}_{\mathbb{C}}\left\{\tilde{a}_{\mathcal{I}_{1}} \cdots \tilde{a}_{\mathcal{I}_{n}}|0\rangle\right\}, \quad a_{\mathcal{I}}|0\rangle=0
$$

Here, $\mathcal{I}$ is a collective index which can be specified in each dual pairs, and the number $n$ of $W_{n}$ stands for the total oscillator number. Since all the states of $\mathcal{W}$ are positive definite, all irreducible representations appearing in the decomposition of $\mathcal{W}$ are unitary. Obtaining this decomposition becomes particularly simple when $K=G$ due to the properties 1 and 2 . From now on, we assume that $G$ is compact.

From the property 1, we know that $G$ commutes with the total number operator, and hence the eigenspace $W_{n}$ can be decomposed into several irreducible representations $\pi_{G}(\zeta)$ :

$$
W_{n}=\bigoplus_{\zeta} \bigoplus_{i=1}^{m_{n}^{\zeta}} V_{n, i}^{\zeta}
$$

where the first sum runs over a finite number of labels $\zeta, m_{n}^{\zeta}$ is the multiplicity of $\pi_{G}(\zeta)$ in $W_{n}$, and $V_{n, i}^{\zeta}$ is the representation space of $\pi_{G}(\zeta)$ in $W_{n}$ with the multiplicity label $i$. The restriction to $\pi_{G}(\zeta)$ in $W_{n}$ therefore reads

$$
\left.W_{n}\right|_{\pi_{G}(\zeta)}=\bigoplus_{i=1}^{m_{n}^{\zeta}} V_{n, i}^{\zeta} .
$$

Consequently, a finite-dimensional irreducible representation $\pi_{G}(\zeta)$ is realized in infinitely many subspaces $W_{n}$ :

$$
\left.\mathcal{W}\right|_{\zeta} ^{G}=\left.\bigoplus_{n \in N_{\zeta}} W_{n}\right|_{\pi_{G}(\zeta)}=\bigoplus_{n \in N_{\zeta}} \bigoplus_{i=1}^{m_{n}^{\zeta}} V_{n, i}^{\zeta},
$$

where $N_{\zeta}$ is the set of integers $n$ such that $\pi_{G}(\zeta)$ appears in $W_{n}$, or equivalently the possible numbers of oscillators with which a state in $\pi_{G}(\zeta)$ can be realized. All $V_{n, i}^{\zeta}$ with $n \in N_{\zeta}$ and $i=1, \ldots, m_{n}^{\zeta}$ have to be related to one another by the dual group $G^{\prime}$, since the decomposition of $\mathcal{W}$ under $G \times G^{\prime}$ is multiplicity-free (i.e. $G^{\prime}$ acts irreducibly on $\left.\mathcal{W}\right|_{\zeta} ^{G}$ ). In particular, the space $\bigoplus_{i=1}^{m_{n}^{\zeta}} V_{n, i}^{\zeta}$ carries a $m_{n}^{\zeta}$-dimensional representation of the maximal compact subgroup $K^{\prime}$ of $G^{\prime}$.

When $G^{\prime}=K^{\prime}$, its generators also commute with the total number operator, and hence they preserve the subspaces $W_{n}$. Consequently, the set $N_{\zeta}$ has only one element and the corresponding $m_{n}^{\zeta}$-dimensional representation $\zeta^{\prime}$ of $G^{\prime}$ is irreducible. In this way, we find the duality between the representations $\zeta$ and $\zeta^{\prime}$ for the dual pairs of the type $\left(K, K^{\prime}\right)$. In fact, the only such case is the $(U(M), U(N))$ duality.

When $G^{\prime} \neq K^{\prime}$, the set $N_{\zeta}$ will have the form,

$$
N_{\zeta}=\left\{n_{\zeta}+2 m \mid m \in \mathbb{N}\right\}
$$


since the generators $G^{\prime}$ are quadratic in the oscillators. Here, $n_{\zeta}$ is the minimum oscillator number with which $\zeta$ is realized. Now, from the property 2, we find that the Lie algebra of $G^{\prime}$ admits the decomposition (5.1) and the lowering operators in $\mathfrak{g}_{-2}^{\prime}$ annihilate the states $V_{n_{\zeta}, i}^{\zeta}$. In this way, we demonstrate that the dual representation $\zeta^{\prime}$ of $G^{\prime}$ is a lowest weight representation. ${ }^{15}$

In the following, we will review the correspondences between the representations of dual pairs, in which at least one member admits a compact real form. This correspondence was originally derived in [81] (see e.g. [28] for a review). Here, we rederive the same results using the oscillator realizations presented above. Notice that a similar treatment can be found in appendix B of [55], though the discussion was at the level of complex Lie algebras. Let us also mention that in [34-42], representations of $U\left(N_{+}, N_{-}\right), S p(2 N, \mathbb{R}), O^{*}(2 N)$ are studied using the same oscillator realizations as the ones used in this paper. However, in these references, the role of the dual pairs and the correspondences of their representations were not explored.

\section{$5.1(U(M), U(N))$}

Let us begin the analysis with the simplest case, the $(U(M), U(N))$ duality. In this case, the groups $U(M)$ and $U(N)$ are respectively generated by

$$
X_{b}^{a}=\tilde{a}_{i}^{a} a_{b}^{i}+\frac{N}{2} \delta_{b}^{a}, \quad R_{j}{ }^{i}=\tilde{a}_{j}^{a} a_{a}^{i}+\frac{M}{2} \delta_{j}^{i},
$$

where $a, b=1, \ldots, M$ and $i, j=1, \ldots, N$. The Fock space $\mathcal{W}$ is generated by polynomials in $\tilde{a}_{i}^{a}$, namely

$$
\mathcal{W}=\bigoplus_{L \in \mathbb{N}} W_{L}, \quad W_{L}:=\operatorname{span}_{\mathbb{C}}\left\{\tilde{a}_{i_{1}}^{a_{1}} \ldots \tilde{a}_{i_{L}}^{a_{L}}|0\rangle\right\}
$$

Any state $|\Psi\rangle \in W_{L}$ can be expressed as

$$
|\Psi\rangle:=\Psi_{a_{1} \cdots a_{L}}^{i_{1} \cdots i_{L}} \tilde{a}_{i_{1}}^{a_{1}} \cdots \tilde{a}_{i_{L}}^{a_{L}}|0\rangle
$$

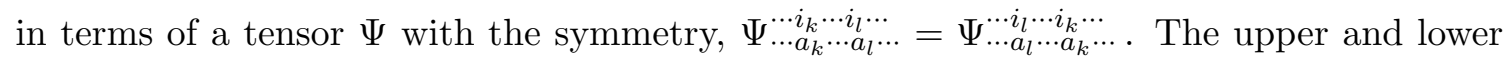
indices of $\Psi$ carry tensor representations $\square^{\otimes L}$ of $U(M)$ and $U(N)$ respectively, where $\square$ denotes their fundamental representation.

Let us first consider the $U(M)$ representation: $\square^{\otimes L}$ can be decomposed into irreducible representations corresponding to the Young diagrams with height not greater than $M$, and we pick up the irreducible representation corresponding to the Young diagram,

$$
\ell=\left(\ell_{1}, \ldots, \ell_{p}\right)
$$

where $\ell_{k}$ is the length of the $k$-th row and $p$ is the height $h(\ell)$ of $\ell$, and $L=|\ell|:=\ell_{1}+\cdots+\ell_{p}$. Since the $U(M)$ generators can be realized in a Fock space with any constant shift, such

\footnotetext{
${ }^{15}$ Notice that in general, $\mathfrak{g}_{-2}$ does not contain all the lowering operators of $\mathfrak{g}$, as some of them are also included in $\mathfrak{g}_{0}$. However, $\bigoplus_{i=1}^{m_{n}^{\zeta}} V_{n_{\zeta}, i}^{\zeta}$ forms a finite-dimensional irreducible representation of the compact group $K$, and is therefore itself a lowest weight representation. This implies that it contains a lowest weight vector, which is also a lowest weight vector for $\mathfrak{g}$ due to the fact that the Cartan subalgebra of the latter coincides with the Cartan subalgebra of $K$.
} 
as $\frac{N}{2} \delta_{b}^{a}$ in (5.7), we need to specify it together with a Young diagram when labeling the $U(M)$ representations. Note that this shift only affects the representation of the diagonal $U(1)$ in $U(M)$, but not the $S U(M)$ part. Taking this into account, let us label the $U(M)$ representation as

$$
\left[\ell, \frac{N}{2}\right]_{U(M)},
$$

which can be decomposed into $U(1) \times S U(M)$ representation as

$$
\left[\ell, \frac{N}{2}\right]_{U(M)}=\left[|\ell|+\frac{N M}{2}\right]_{U(1)} \otimes[\ell]_{S U(M)},
$$

where $|\ell|+N M / 2$ is the eigenvalue of the $U(1)$ generator. In this way, we can produce all $U(M)$ representations using an oscillator realization. The highest weight of $\left[\ell, \frac{N}{2}\right]_{U(M)}$ simply reads

$$
\left(\ell_{1}+\frac{N}{2}, \ell_{2}+\frac{N}{2}, \ldots, \ell_{M}+\frac{N}{2}\right)
$$

Now, let us see to which $U(N)$ representation the $\left[\ell, \frac{N}{2}\right]_{U(M)}$ representation corresponds. We can do that by picking up a particular state in the $\left[\ell, \frac{N}{2}\right]_{U(M)}$ representation - which consists of multiple states $|\Psi\rangle$ in $W_{L}$ - and reading off the $U(N)$ representation from such states $|\Psi\rangle$. Let us pick up the lowest weight state in the $\left[\ell, \frac{N}{2}\right]_{U(M)}$ representation. By acting with the lowering operators $\left(X_{b}^{a}\right.$ with $\left.a<b\right)$ of $U(M)$, we bring $|\Psi\rangle$ to the lowest weight state of $\left[\ell, \frac{N}{2}\right]_{U(M)}$, corresponding to the Young tableau,

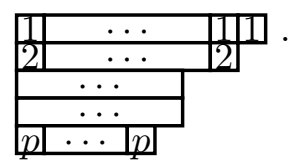

Such states $|\Psi\rangle$ satisfy

$$
X_{b}^{a}|\Psi\rangle=0 \quad[1 \leq a<b \leq p] .
$$

To identify the solution space of the above condition, it will be convenient to first re-express the state $|\Psi\rangle$ or the tensor $\Psi$ as

$$
|\Psi\rangle=\Psi^{i_{1}\left(\ell_{1}\right), \ldots, i_{l}\left(\ell_{p}\right)} \tilde{a}_{i_{1}\left(\ell_{1}\right)}^{1} \cdots \tilde{a}_{i_{p}\left(\ell_{p}\right)}^{p}|0\rangle,
$$

where $\Psi^{\cdots i_{k}\left(\ell_{k}\right) \cdots}$ and $\tilde{a}_{i_{k}\left(\ell_{k}\right)}^{k}$ are defined by

$$
\begin{gathered}
\Psi^{\cdots i_{k}\left(\ell_{k}\right) \cdots}:=\Psi^{\cdots \overbrace{i_{k} \cdots i_{k}}^{\ell_{k}} \cdots}:=\Psi^{\cdots}\left(_{i}^{1} \cdots i_{k}^{\ell_{k}}\right) \cdots, \\
\tilde{a}_{i_{k}\left(\ell_{k}\right)}^{k}:=\overbrace{\tilde{a}_{i_{k}}^{k} \cdots \tilde{a}_{i_{k}}^{k}}^{\ell_{k}}:=\tilde{a}_{\left(i_{k}^{1}\right.}^{k} \cdots \tilde{a}_{\left.i_{k} \ell_{k}\right)}^{k} .
\end{gathered}
$$

Then, the condition (5.15) is translated to

$$
\Psi^{i_{1}\left(\ell_{1}\right), \ldots, i_{a}\left(\ell_{a}\right), \ldots, i_{a} i_{b}\left(\ell_{b}-1\right), \ldots, i_{p}\left(\ell_{p}\right)}=0 \quad[1 \leq a<b \leq p] .
$$


To rephrase the above condition in words, the symmetrization of the $\ell_{a}$ indices in the $a$-th group with one index $i_{b}$ in the $b$-th group vanishes identically. This is nothing but the definition of the same Young diagram $\ell$, but this time it designates $U(N)$ representation,

$$
\left[\ell, \frac{M}{2}\right]_{U(N)} \text {. }
$$

Therefore, we find the correspondence of the representations:

$$
\left[\ell, \frac{M}{2}\right]_{U(N)} \quad \longleftrightarrow \quad\left[\ell, \frac{N}{2}\right]_{U(M)},
$$

where the height $h(\ell)$ of the Young diagram should be bounded by both $M$ and $N: h(\ell) \leq$ $\min \{M, N\}$.

\section{$5.2\left(U\left(M_{+}, M_{-}\right), U(N)\right)$}

The dual pair $\left(U\left(M_{+}, M_{-}\right), U(N)\right)$ is realized as

$$
\begin{aligned}
X_{b}^{a}{ }_{b} & =\tilde{a}_{i}^{a} a_{b}^{i}+\frac{N}{2} \delta_{b}^{a}, \quad X_{\mathrm{b}}^{\mathrm{a}}=-\tilde{b}_{\mathrm{b}}^{i} b_{i}^{\mathrm{a}}-\frac{N}{2} \delta_{\mathrm{b}}^{\mathrm{a}}, \quad X_{\mathrm{b}}^{a}=-\tilde{a}_{i}^{a} \tilde{b}_{\mathrm{b}}^{i}, \quad X_{b}^{\mathrm{a}}=a_{b}^{i} b_{i}^{\mathrm{a}}, \\
R_{j}{ }^{i} & =\tilde{a}_{j}^{a} a_{a}^{i}-\tilde{b}_{\mathrm{a}}^{i} b_{j}^{\mathrm{a}}+\frac{M_{+}-M_{-}}{2} \delta_{j}^{i},
\end{aligned}
$$

where $a, b=1, \ldots, M_{+}, \mathrm{a}, \mathrm{b}=1, \ldots, \mathrm{M}_{-}$and $i, j=1, \ldots, N$. The Fock space $\mathcal{W}$ is generated by polynomials in two families of oscillators, namely

$$
\mathcal{W}=\bigoplus_{L, M \in \mathbb{N}} W_{L, M}, \quad W_{L, M}:=\operatorname{span}_{\mathbb{C}}\left\{\tilde{a}_{i_{1}}^{a_{1}} \ldots \tilde{a}_{i_{L}}^{a_{L}} \tilde{b}_{\mathrm{a}_{1}}^{j_{1}} \ldots \tilde{b}_{\mathrm{a}_{M}}^{j_{M}}|0\rangle\right\}
$$

Recall for a dual pair $\left(G, K^{\prime}\right)$ with $K^{\prime}$ a compact Lie group, the realization of a finitedimensional irrep of $K^{\prime}$ with the minimal number of oscillators in $\mathcal{W}$ also forms an irreducible representation of the maximal compact subgroup $K \subset G$. For $G=U\left(M_{+}, M_{-}\right)$, the maximal compact subgroup is $U\left(M_{+}\right) \times U\left(M_{-}\right)$, represented by $X^{a}{ }_{b}$ and $X^{\mathrm{a}}{ }_{\mathrm{b}}$. Let us pick up a $K=U\left(M_{+}\right) \times U\left(M_{-}\right)$representation,

$$
\left[\boldsymbol{\ell}, \frac{N}{2}\right]_{U\left(M_{+}\right)} \otimes\left[\boldsymbol{m}, \frac{N}{2}\right]_{U\left(M_{-}\right)} .
$$

According to the seesaw duality,

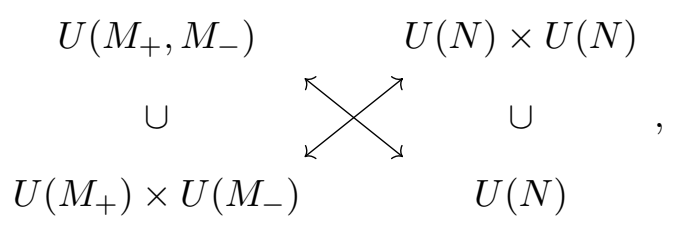

where $U(N) \times U(N)$ are generated by $\tilde{a}_{j}^{a} a_{a}^{i}+\frac{M_{+}}{2} \delta_{j}^{i}$ and $\tilde{b}_{\mathrm{a}}^{i} b_{j}^{\mathrm{a}}+\frac{M_{-}}{2} \delta_{j}^{i}$, the representation of $U(N) \times U(N)$ dual to (5.24) will be

$$
\left[\ell, \frac{M_{+}}{2}\right]_{U(N)} \otimes\left[\boldsymbol{m}, \frac{M_{-}}{2}\right]_{U(N)} .
$$


A lowest weight state $|\Psi\rangle$ of (5.24) can be expressed as

$$
|\Psi\rangle=\Psi_{j_{1}\left(m_{1}\right), \ldots, j_{q}\left(m_{q}\right)}^{i_{1}\left(\ell_{1}\right), \ldots, i_{p}\left(\ell_{p}\right)} \tilde{a}_{i_{1}\left(\ell_{1}\right)}^{1} \cdots \tilde{a}_{i_{p}\left(\ell_{p}\right)}^{p} \tilde{b}_{1}^{j_{1}\left(m_{1}\right)} \cdots \tilde{b}_{\mathrm{q}}^{j_{q}\left(m_{q}\right)}|0\rangle
$$

where the upper and lower indices of $\Psi$ have the symmetries of the Young diagram $\ell$ and $\boldsymbol{m}$ respectively, with $h(\boldsymbol{\ell})=p$ and $h(\boldsymbol{m})=q$. Such a state is annihilated by $X_{a}^{\mathrm{b}}$, as these generators decrease the total oscillator number, and $|\Psi\rangle$ is a state with the lowest number of oscillator in the irreducible representations of $U\left(M_{+}, M_{-}\right)$and $U(N)$. This condition is translated to

$$
X_{a}^{\mathrm{b}}|\Psi\rangle=0 \quad \Leftrightarrow \quad \delta_{i_{a}}^{j_{b}} \Psi_{j_{1}\left(m_{1}\right), \ldots, j_{q}\left(m_{q}\right)}^{i_{1}\left(\ell_{1}\right), \ldots, i_{p}\left(\ell_{p}\right)}=0 .
$$

In words, the $\Psi$ tensor is traceless in the sense that any contraction between upper and lower indices vanishes identically. This defines the irreducible representation $\left[\boldsymbol{\ell} \oslash \boldsymbol{m}, \frac{M_{+}-M_{-}}{2}\right]_{U(N)}$ of $U(N)$ whose highest weight is

$$
\left(\ell_{1}, \ldots, \ell_{p}, 0, \ldots, 0,-m_{q}, \ldots,-m_{1}\right)+\frac{M_{+}-M_{-}}{2}(1, \ldots, 1) .
$$

See appendix A for more details on general $U(N)$ representations. In this way, we see that the $U\left(M_{+}\right) \times U\left(M_{-}\right)$representation $\left[\ell, \frac{N}{2}\right]_{U\left(M_{+}\right)} \otimes\left[\boldsymbol{m}, \frac{N}{2}\right]_{U\left(M_{-}\right)}$induces a lowest weight $U\left(M_{+}, M_{-}\right)$representation, while the $U(N) \times U(N)$ representation $\left[\ell, \frac{M_{+}}{2}\right]_{U(N)} \otimes$ $\left[\boldsymbol{m}, \frac{M_{-}}{2}\right]_{U(N)}$ is restricted to the $U(N)$ representation $\left[\boldsymbol{\ell} \oslash \boldsymbol{m}, \frac{M_{+}-M_{-}}{2}\right]_{U(N)}$. In the end, we find the following correspondence,

$$
\mathcal{D}_{U\left(M_{+}, M_{-}\right)}\left(\left[\boldsymbol{\ell}, \frac{N}{2}\right]_{U\left(M_{+}\right)} \otimes\left[\boldsymbol{m}, \frac{N}{2}\right]_{U\left(M_{-}\right)}\right) \quad \longleftrightarrow \quad\left[\boldsymbol{\ell} \oslash \boldsymbol{m}, \frac{M_{+}-M_{-}}{2}\right]_{U(N)} .
$$

For $M_{+}=M_{-}=1$, due to the isomorphism between $U(1,1)$ and $U(1) \times S L(2, \mathbb{R})$ representations, we can write

$$
\mathcal{D}_{U(1,1)}\left(\left[\ell+\frac{N}{2}\right]_{U(1)} \otimes\left[m+\frac{N}{2}\right]_{U(1)}\right)=[\ell-m]_{U(1)} \otimes \mathcal{D}_{S L(2, \mathbb{R})}(\ell+m+N),
$$

where $\mathcal{D}_{S L(2, \mathbb{R})}(h)$ denote the positive discrete series representation of $S L(2, \mathbb{R})$ with lowest weight $h \in \mathbb{N}$.

Let us briefly comment on an example, which is important in physics. The doublecover of the four-dimensional conformal group $\widetilde{S O^{+}}(2,4)$ is isomorphic to $S U(2,2)$ so that the representation $\mathcal{D}_{U(2,2)}\left(\left[\left(\ell_{1}, \ell_{2}\right), \frac{N}{2}\right]_{U(2)} \otimes\left[\left(m_{1}, m_{2}\right), \frac{N}{2}\right]_{U(2)}\right)$ corresponds to the representation $[2 h]_{U(1)} \otimes \mathcal{D}_{\widetilde{S O}^{+}(2,4)}\left(\Delta ; s_{1}, s_{2}\right)$ with

$$
\begin{aligned}
h & =\frac{1}{2}\left(\ell_{1}+\ell_{2}-m_{1}-m_{2}\right), & \Delta & =\frac{1}{2}\left(\ell_{1}+\ell_{2}+m_{1}+m_{2}\right)+N, \\
s_{1} & =\frac{1}{2}\left(\ell_{1}-\ell_{2}+m_{1}-m_{2}\right), & s_{2} & =\frac{1}{2}\left(\ell_{1}-\ell_{2}-m_{1}+m_{2}\right) .
\end{aligned}
$$

The $\widetilde{S O^{+}}(2,4)$ representations appearing in this duality describe $\mathrm{CFT}_{4}$ operators or equivalently fields in $\mathrm{AdS}_{5}$. In light of the previous dictionary, it will be convenient to parametrize the $U(N)$ irreps $[\ell \oslash \boldsymbol{m}]_{U(N)}$ appearing in this correspondence as

$$
\boldsymbol{\ell}=\left(s_{1}+s_{2}+n, n\right), \quad \boldsymbol{m}=\left(s_{1}-s_{2}+k, k\right),
$$


with $k, n \in \mathbb{N}$ and $s_{i}$ satisfying

$$
s_{1}, s_{2} \in \frac{1}{2} \mathbb{Z}, \quad s_{1} \geq\left|s_{2}\right| .
$$

Moreover, $s_{1}$ and $s_{2}$ are either both integers or bother half-integers. Note that $s_{1}$ is positive while $s_{2}$ can be positive or negative. The correspondence (5.30) then reads

$$
\begin{gathered}
{\left[\left(s_{1}+s_{2}+n, n\right) \oslash\left(s_{1}-s_{2}+k, k\right)\right]_{U(N)}} \\
\downarrow \\
{\left[2\left(s_{2}+n-k\right)\right]_{U(1)} \otimes \mathcal{D}_{\widetilde{S O}^{+}(2,4)}\left(s_{1}+n+k+N ; s_{1}, s_{2}\right) .}
\end{gathered}
$$

For low values of $N$, the $U(N)$ labels $(5.33)$ are restricted and the dual $\widetilde{S O^{+}}(2,4)$ representations correspond to particular fields:

- $N=1$ : we should require $k=n=0$ and $s_{1}= \pm s_{2}$, so that the correspondence reads

$$
[ \pm 2 s]_{U(1)} \longleftrightarrow \quad[ \pm 2 s]_{U(1)} \otimes \mathcal{D}_{\widetilde{S O}^{+}(2,4)}(s+1 ; s, \pm s)
$$

These $\widetilde{S O^{+}}(2,4)$ representations describes four-dimensional massless fields of helicity $\pm s$, also known as singletons.

- $N=2$ : there are two types of $U(2)$ irreps that can occur.

The first one characterized by $n=k=0$, and for which the correspondence reads

$$
\left[\left(s_{1}+s_{2}, s_{2}-s_{1}\right)\right]_{U(2)} \longleftrightarrow \quad\left[2 s_{2}\right]_{U(1)} \otimes \mathcal{D}_{\widetilde{S O}^{+}(2,4)}\left(s_{1}+2 ; s_{1}, s_{2}\right)
$$

These $\widetilde{S O^{+}}(2,4)$ representation correspond to $\mathrm{CFT}_{4}$ conserved current of $\operatorname{spin}\left(s_{1}, s_{2}\right)$, or equivalently to massless fields in $\mathrm{AdS}_{5}$ with the same spin.

The second type is characterized by either $k=0$ and $s_{1}=s_{2}=s$, or $n=0$ and $s_{1}=-s_{2}=s$, and the correspondence reads

$$
\begin{aligned}
{[(2 s+n, n)]_{U(2)} } & \leftrightarrow[2(s+n)]_{U(1)} \otimes \mathcal{D}_{\widetilde{S O}^{+}(2,4)}(s+n+2 ; s, s) \\
{[(-n,-2 s-n)]_{U(2)} } & \leftrightarrow \quad[-2(s+n)]_{U(1)} \otimes \mathcal{D}_{\widetilde{S O}^{+}(2,4)}(s+n+2 ; s,-s) .
\end{aligned}
$$

These $\widetilde{S O^{+}}(2,4)$ representations correspond to all possible $\mathrm{CFT}_{4}$ operators with spin $(s, \pm s)$ and twist $\tau=\Delta-s_{1} \geq 2$, or equivalently massive $\mathrm{AdS}_{5}$ fields with the same spin.

As expected, these are all the possible representations appearing in the decomposition of the tensor product of two singletons, which was given in [82].

- $N=3$ : in this case, the $U(3)$ irrep labelled by (5.33) appearing in the decomposition are such that either $n=0$ or $k=0$, and the correspondence reads

$$
\begin{aligned}
{\left[\left(s_{1}+s_{2}+n, n, s_{2}-s_{1}\right)\right]_{U(3)} } & \leftrightarrow\left[2\left(s_{2}+n\right)\right]_{U(1)} \otimes \mathcal{D}_{\widetilde{S O}^{+}(2,4)}\left(s_{1}+n+3 ; s_{1}, s_{2}\right) \\
{\left[\left(s_{1}+s_{2},-n, s_{2}-s_{1}-n\right)\right]_{U(3)} } & \leftrightarrow\left[2\left(s_{2}-n\right)\right]_{U(1)} \otimes \mathcal{D}_{\widetilde{S O}^{+}(2,4)}\left(s_{1}+n+3 ; s_{1}, s_{2}\right)
\end{aligned}
$$


These $\widetilde{S O^{+}}(2,4)$ representations describe massive fields in $\operatorname{AdS}_{5}$ with spin $\left(s_{1}, s_{2}\right)$ or $\mathrm{CFT}_{4}$ operators with twist $\tau \geq 3$.

- $N \geq 4$ : no restriction is to be imposed on the $U(N)$ labels (5.33) and the dual representations correspond to massive $\mathrm{AdS}_{5}$ fields of any spin or $\mathrm{CFT}_{4}$ operators with twist $\tau \geq N$.

Notice that in $[35,42]$ the oscillator realization of $S U(2,2 \mid 4)$ was obtained and some tensor product decompositions of singletons were analyzed. This dual pair correspondence was also used in the context of $\mathrm{AdS}_{5} / \mathrm{CFT}_{4}$ for higher spin gauge theory in [51]. More recently its relevance as a possible tool for computing scattering amplitudes was pointed out in [83, 84].

$\mathbf{5 . 3}(O(N), S p(2 M, \mathbb{R}))$

The dual pair $(O(N), S p(2 M, \mathbb{R}))$ is realized as

$$
\begin{aligned}
M_{a b} & =\tilde{a}_{i}^{a} a_{b}^{i}-\tilde{a}_{i}^{b} a_{a}^{i}, \\
K^{+i+j} & =a_{a}^{i} a_{a}^{j}, \quad K^{-i-j}=\tilde{a}_{i}^{a} \tilde{a}_{j}^{a}, \quad K^{+i-j}=\tilde{a}_{j}^{a} a_{a}^{i}+\frac{N}{2} \delta_{j}^{i},
\end{aligned}
$$

where $a, b=1, \ldots N$ and $i, j=1, \ldots M$, and the Fock space $\mathcal{W}$ is spanned by polynomials in one family of oscillators, namely

$$
\mathcal{W}=\bigoplus_{L \in \mathbb{N}} W_{L}, \quad W_{L}:=\operatorname{span}_{\mathbb{C}}\left\{\tilde{a}_{i_{1}}^{a_{1}} \ldots \tilde{a}_{i_{L}}^{a_{L}}|0\rangle\right\}
$$

Similarly to the previous section, we can use the seesaw duality,

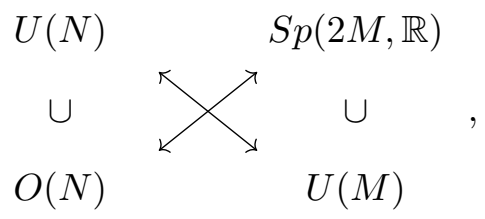

where $U(N)$ is generated by $\tilde{a}_{i}^{a} a_{b}^{i}$ and $U(M)$ is the maximal compact subgroup of $S p(2 M, \mathbb{R})$ generated by $K^{+i-j}$. The dual pair $(U(N), U(M))$ gives the correspondence of the representations,

$$
[\ell, 0]_{U(N)} \longleftrightarrow \quad\left[\ell, \frac{N}{2}\right]_{U(M)} .
$$

This means that by considering the lowest weight state of $[\ell, 0]_{U(M)}$ like $(5.14)$, the state $|\Psi\rangle$ can be expressed as

$$
|\Psi\rangle=\Psi_{a_{1}\left(\ell_{1}\right), \ldots, a_{p}\left(\ell_{p}\right)} \tilde{a}_{1}^{a_{1}\left(\ell_{1}\right)} \ldots \tilde{a}_{p}^{a_{p}\left(\ell_{p}\right)}|0\rangle,
$$

and the lower indices of $\Psi$ have the symmetries of the Young diagram $\ell$ with $h(\ell)=p$, indicating the $[\ell, 0]_{U(N)}$ representation. The lowest $K=U(M)$ condition on these states reads

$$
K^{+i+j}|\Psi\rangle=0 \quad \Leftrightarrow \quad \delta^{a_{i} a_{j}} \Psi_{a_{1}\left(\ell_{1}\right), \ldots, a_{p}\left(\ell_{p}\right)}=0
$$


meaning that $\Psi$ is a traceless tensor. The traceless condition imposes $\ell^{1}+\ell^{2} \leq N$ besides $\ell^{1} \leq N$. The traceless Young diagram $\ell$ specifies a unique irreducible representation of $O(N)$ :

$$
\mathcal{D}_{S p(2 M, \mathbb{R})}\left(\left[\ell, \frac{N}{2}\right]_{U(M)}\right) \quad \longleftrightarrow \quad[\ell]_{O(N)},
$$

where $\ell^{1} \leq \min (M, N)$ and $\ell^{1}+\ell^{2} \leq N$.

For $M=1$, we can use the isomorphism $S p(2, \mathbb{R}) \cong S L(2, \mathbb{R})$ and find the discrete series,

$$
\mathcal{D}_{S L(2, \mathbb{R})}\left(\ell+\frac{N}{2}\right) \quad \longleftrightarrow \quad[(\ell)]_{O(N)}
$$

The $N=1,2$ and 3 cases cover all available discrete series representations. In contrast with $(U(1,1), U(M))$ dual pairs, the $(S p(2, \mathbb{R}), O(N))$ pairs also give the irreps with half-integral lowest weights for odd $N$, which represent the double cover $\widetilde{S L}(2, \mathbb{R})$.

Notice that the above correspondence remains valid even for $N=1$, where the $O(1) \cong$ $\mathbb{Z}_{2}$ representation just reduces to a sign $[ \pm 1]_{\mathbb{Z}_{2}}$, and we find the duality,

$$
\mathcal{D}_{S p(2 M, \mathbb{R})}\left(\left[(\bar{n}), \frac{1}{2}\right]_{U(M)}\right) \quad \longleftrightarrow \quad[(\bar{n})]_{O(1)}=\left[(-1)^{\bar{n}}\right]_{\mathbb{Z}_{2}}
$$

where $\bar{n}=0$ or 1 . In terms of highest weight, the former gives

$$
\mathcal{D}_{S p(2 M, \mathbb{R})}\left(\frac{1}{2}, \frac{1}{2}, \ldots, \frac{1}{2}\right) \quad \text { or } \quad \mathcal{D}_{S p(2 M, \mathbb{R})}\left(\frac{3}{2}, \frac{1}{2}, \ldots, \frac{1}{2}\right),
$$

which is in accordance with the discussion of section 2.1, and in particular (2.8).

Let us briefly comment on an example, which is important in physics. The double cover of the three dimensional conformal group $\widetilde{S O}+(2,3)$ is isomorphic to $S p(4, \mathbb{R})$. Consequently, the lowest weight representation of $\mathcal{D}_{S p(4, \mathbb{R})}\left(\left[\left(\ell_{1}, \ell_{2}\right), \frac{N}{2}\right]\right)$ corresponds to the $\widetilde{S O^{+}}(2,3)$ representation $\mathcal{D}_{\widetilde{S O}^{+}(2,3)}(\Delta ; s)$ with conformal dimension/minimal energy $\Delta$ and spin- $s$ given by

$$
\Delta=\frac{1}{2}\left(\ell_{1}+\ell_{2}+N\right), \quad s=\frac{1}{2}\left(\ell_{1}-\ell_{2}\right) .
$$

Using this relation, we can interpret the $S p(4, \mathbb{R})$ irreps appearing in the dual pair correspondence $(S p(4, \mathbb{R}), O(N))$ as a spectrum of conformal fields in three dimensions, or fields in $\mathrm{AdS}_{4}$. A convenient way to parametrize the irreps $[\boldsymbol{\ell}]_{O(N)}$ appearing in (5.47) is

$$
\ell=(2 s+n, n)
$$

with $s \in \frac{1}{2} \mathbb{N}$ and $n \in \mathbb{N}$, so that the correspondence reads

$$
[(2 s+n, n)]_{O(N)} \quad \longleftrightarrow \quad \mathcal{D}_{\widetilde{S O}^{+}(2,3)}\left(s+n+\frac{N}{2} ; s\right)
$$

For low values of $N$, not all two-row Young diagrams define a representation of $O(N)$ : 
- $N=1$ : we find the correspondence,

$$
[(0)]_{O(1)} \leftrightarrow \quad \mathcal{D}_{\widetilde{S O}^{+}(2,3)}\left(\frac{1}{2} ; 0\right) \quad \text { and } \quad[(1)]_{O(1)} \quad \leftrightarrow \quad \mathcal{D}_{\widetilde{S O}^{+}(2,3)}\left(1 ; \frac{1}{2}\right)
$$

where the $\widetilde{S O^{+}}(2,3)$ representations are respectively isomorphic to the spin-0 singleton (or Rac) and spin- $\frac{1}{2}$ singleton (or Di). Originally discovered by Dirac [15], they describe a free conformal scalar and spinor respectively, in three dimensions.

- $N=2$ : the $O(2)$ irreps are labelled by a Young diagram which is either a single row of length $2 s$ (i.e. $n=0$ ), or a single column of height two (i.e. $s=n=1$ ). For a single row diagram, we have

$$
[(2 s)]_{O(2)} \longleftrightarrow \quad \mathcal{D}_{\widetilde{S O}^{+}(2,3)}(s+1 ; s)
$$

and the $\widetilde{S O^{+}}(2,3)$ representation describes a spin- $s$ conserved current in three dimensions or a spin- $s$ massless field in $\mathrm{AdS}_{4}$, for $s>\frac{1}{2}$. For $s=0$ (resp. $\frac{1}{2}$ ), this representation describes a scalar (resp. spinor) $\mathrm{CFT}_{3}$ operator / $\mathrm{AdS}_{4}$ field with conformal dimension / minimal energy 1 (resp. $\frac{3}{2}$ ). For the single column diagram, we have

$$
[(1,1)]_{O(2)} \longleftrightarrow \quad \mathcal{D}_{\widetilde{S O}^{+}(2,3)}(2 ; 0)
$$

and the $\widetilde{S O^{+}}(2,3)$ representation describes a scalar $\mathrm{CFT}_{3}$ operator / $\mathrm{AdS}_{4}$ field with conformal dimension / minimal energy 2. As expected, this spectrum is that of the decomposition of the tensor product of two singletons, first derived in [85] (later revisited and extended to arbitrary dimensions in $[53,82,86])$.

- $N=3$ : in this case, we can only consider $n=0$ or $n=1$ so that the correspondence reads

$$
[(2 s)]_{O(3)} \longleftrightarrow \quad \mathcal{D}_{S O(2,3)}\left(s+\frac{3}{2} ; s\right)
$$

or

$$
[(2 s+1,1)]_{O(3)} \quad \longleftrightarrow \quad \mathcal{D}_{\widetilde{S O}^{+}(2,3)}\left(s+\frac{5}{2} ; s\right)
$$

In other words, all operators of twist $\tau=\frac{3}{2}$ and $\tau=\frac{5}{2} /$ massive fields with spin- $s$ and minimal energy $s+\frac{3}{2}$ or $s+\frac{5}{2}$ appear.

- $N \geq 4$ : no restriction is to be imposed on the $O(N)$ labels and the corresponding $\widetilde{S O}+(2,3)$ representations describe all operators with twist $\tau=k+\frac{N}{2} /$ massive fields with spin- $s$ and minimal energy $s+k+\frac{N}{2}$.

Notice that the spectrum of the $N$-th tensor product of three-dimensional singletons was obtained already in [87] using the same oscillator realization (see also [36, 88] and references therein for earlier works). 


\section{$5.4\left(O^{*}(2 N), S p(M)\right)$}

The dual pair $\left(O^{*}(2 N), S p(M)\right)$ is realized as

$$
\begin{aligned}
M_{+a-b} & =\tilde{a}_{a}^{R} a_{R}^{b}+M \delta_{a b}, \quad M_{+a+b}=2 \tilde{a}_{[a}^{R} \tilde{a}_{b] R}, \quad M_{-a-b}=2 a^{R[a} a_{R}^{b]}, \\
K^{R S} & =2 \tilde{a}_{a}^{(R} a^{S) a} .
\end{aligned}
$$

where $a, b=1, \ldots, n$ and $R, S=1, \ldots, 2 M$. The Fock space $\mathcal{W}$ is spanned by polynomials in one family of oscillators, namely

$$
\mathcal{W}=\bigoplus_{L \in \mathbb{N}} W_{L}, \quad W_{L}:=\operatorname{span}_{\mathbb{C}}\left\{\tilde{a}_{a_{1}}^{R_{1}} \ldots \tilde{a}_{a_{L}}^{R_{L}}|0\rangle\right\}
$$

We use again the seesaw duality,

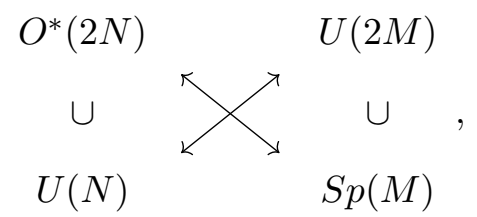

where $U(N)$ and $U(2 M)$ are generated by $M_{+a-b}$ and $\tilde{a}_{a}^{R} a_{S}^{a}$ respectively. The dual pair $(U(N), U(2 M))$ gives the correspondence,

$$
[\ell, M]_{U(N)} \longleftrightarrow \quad[\ell, 0]_{U(2 M)}
$$

This means that by considering the lowest weight state of $[\ell, M]_{U(N)}$ like (5.14), the state $|\Psi\rangle$ can be expressed as

$$
|\Psi\rangle=\Psi_{R_{1}\left(\ell_{1}\right), \ldots, R_{p}\left(\ell_{p}\right)} \tilde{a}_{1}^{R_{1}\left(\ell_{1}\right)} \ldots \tilde{a}_{p}^{R_{p}\left(\ell_{p}\right)}|0\rangle,
$$

and the indices of $\Psi$ have the symmetries of the Young diagram $\ell$ with $h(\ell)=p$, indicating the $[\ell, 0]_{U(2 M)}$ representation. The lowest $K=U(N)$ condition on these states reads

$$
M_{-a-b}|\Psi\rangle=0 \quad \Leftrightarrow \quad \Omega^{R_{a} R_{b}} \Psi_{R_{1}\left(\ell_{1}\right), \ldots, R_{p}\left(\ell_{p}\right)}=0,
$$

meaning that the tensor $\Psi$ is traceless in the symplectic sense. The symplectic traceless condition imposes the stronger condition $\ell^{1} \leq M$ than the usual one $\ell^{1} \leq 2 M$. The symplectic traceless Young diagram $\ell$ specifies a unique irreducible representation of $S p(M)$, hence we find the correspondence,

$$
\mathcal{D}_{O^{*}(2 N)}\left([\boldsymbol{\ell}, M]_{U(N)}\right) \quad \longleftrightarrow \quad[\ell]_{S p(M)},
$$

where $p \leq \min \{N, M\}$.

Let us consider the $N=1,2$ cases. When $N=1$, we have the correspondence,

$$
[\ell+M]_{O^{*}(2)} \cong U(1) \quad \longleftrightarrow \quad[(\ell)]_{S p(M)},
$$


between the finite-dimensional representations, due to the isomorphism $O^{*}(2) \cong U(1)$. When $N=2$, we have the isomorphism $O^{*}(4) \cong(S U(2) \times S L(2, \mathbb{R})) / \mathbb{Z}_{2}$ where $S U(2)$ is the special subgroup of the maximal compact subgroup $U(2)$, and $S L(2, \mathbb{R})$ is generated by

$$
\begin{aligned}
H & =\left(M_{+1-1}+M_{+2-2}\right)=\tilde{a}_{1}^{R} a_{R}^{1}+\tilde{a}_{2}^{R} a_{R}^{2}+2 M, \\
E & =M_{+1+2}=\tilde{a}_{1}^{R} \tilde{a}_{R 2}, \\
F & =-M_{-1-2}=-a^{R 1} a_{R}^{2},
\end{aligned}
$$

with the usual Lie brackets,

$$
[H, E]=2 E, \quad[H, F]=-2 F, \quad[E, F]=H .
$$

In terms of $S U(2) \times S L(2, \mathbb{R})$ irreps, the $O^{*}(4)$ representation reduces to

$$
\mathcal{D}_{O^{*}(4)}\left(\left[\left(\ell_{1}, \ell_{2}\right), M\right]_{U(2)}\right)=\left[\ell_{1}-\ell_{2}\right]_{S U(2)} \otimes \mathcal{D}_{S L(2, \mathbb{R})}\left(\ell_{1}+\ell_{2}+2 M\right),
$$

where $\left[\ell_{1}-\ell_{2}\right]_{S U(2)}$ is the $\left(\ell_{1}-\ell_{2}+1\right)$-dimensional representation, and with the understanding that $\ell_{2}=0$ when $M=1$.

Let us briefly comment on an example, which is important in physics. The doublecover of the six-dimensional conformal group $\widetilde{S O^{+}}(2,6)$ and $O^{*}(8)$ are isomorphic. The lowest weight representation $O^{*}(8)$ corresponds to that of $\widetilde{S O^{+}}(2,6)$ as

$$
\begin{gathered}
{\left[\left(s_{1}+s_{2}+s_{3}+n, s_{1}+n, s_{2}+n, s_{3}+n\right)\right]_{S p(M)}} \\
\downarrow \\
\mathcal{D}_{\widetilde{S O}^{+}(2,6)}\left(s_{1}+s_{2}+s_{3}+2 n+2 M ; s_{1}, s_{2}, \pm s_{3}\right),
\end{gathered}
$$

where $n, s_{i}$ satisfy

$$
n, s_{1}, s_{2}, s_{3} \in \frac{1}{2} \mathbb{Z}, \quad s_{1} \geq s_{2} \geq\left|s_{3}\right|, \quad n \geq-s_{3} .
$$

and either $n, s_{1}, s_{2}, s_{3}$ are all integers or all half-integers. The \pm sign represents two possible isomorphisms between $O^{*}(8)$ and $\widetilde{S O^{+}}(2,6)$, which reflects the possibility of parity transformation. Note however that an oscillator realization of $\widetilde{S O}+(2,6)$ provide representations with + or - sign but not both.

For low values of $M$, a few representations are allowed for $S p(M)$ :

- $M=1$ : only one row Young diagram is allowed for $\ell$, and we find the correspondence,

$$
[(2 s)]_{S p(1)} \longleftrightarrow \quad \mathcal{D}_{\widetilde{S O}^{+}(2,6)}(s+2 ; s, s, \pm s)
$$

The $\widetilde{S O^{+}}(2,6)$ representations describe the six-dimensional spin- $s$ singletons. Notice that only positive helicity singletons appear in the decomposition of the Fock space.

- $M=2$ : the height of $\boldsymbol{\ell}$ is at most two, and we find the correspondence,

$$
\left[\left(s_{1}+s_{2}, s_{1}-s_{2}\right)\right]_{S p(2)} \longleftrightarrow \mathcal{D}_{\widetilde{S O}^{+}(2,6)}\left(s_{1}+4 ; s_{1}, s_{2}, \pm s_{2}\right)
$$


The $\widetilde{S O^{+}}(2,6)$ representation describes a conserved current with spin $\left(s_{1}, s_{2}\right)$ in six dimensions or a massless field with the same spin in $\mathrm{AdS}_{7}$. Here again, this coincides with the spectrum of the tensor product of two higher-spin singletons (of the same chirality), which was worked out in [82].

- $M=3$ : the height of $\boldsymbol{\ell}$ is at most three, and we find the correspondence,

$$
\left[\left(s_{1}+s_{2}, s_{1}-s_{3}, s_{2}-s_{3}\right)\right]_{S p(3)} \quad \longleftrightarrow \quad \mathcal{D}_{\widetilde{S O}^{+}(2,6)}\left(s_{1}+s_{2}-s_{3}+6 ; s_{1}, s_{2}, \pm s_{3}\right)
$$

The $\widetilde{S O^{+}}(2,6)$ representation describe $\mathrm{CFT}_{6}$ operators with spin $\left(s_{1}, s_{2}, \pm s_{3}\right)$ and conformal weight $s_{1}+s_{2}-s_{3}+6$ or massive fields in $\mathrm{AdS}_{7}$ with the same spin.

- $M \geq 4$ : no restriction is to be imposed on the $S p(M)$ labels, and the corresponding $\widetilde{S O}(2,6)$ describes all $6 \mathrm{~d}$ operators with twist $\tau \geq 2 M$ or the corresponding massive $\mathrm{AdS}_{7}$ fields.

The oscillator realization of $O^{*}(8)$ in relation with $\mathrm{AdS}_{7} / \mathrm{CFT}_{6}$ was previously considered in e.g. $[39,51,89]$.

\section{Representation of exceptionally compact dual pairs}

In this section, we consider two dual pairs wherein one member becomes compact or discrete for an exceptional reason: the Lie groups $O^{*}(2 N)$ and $O(N, \mathbb{C})$ become compact and discrete, respectively, only for $N=1$. As we will discuss next, these cases require to consider an outer automorphism of the embedding symplectic group in order to make sense of the reductive dual pair correspondence.

\section{$6.1\left(O^{*}(2), S p\left(M_{+}, M_{-}\right)\right)$}

The dual pair $\left(O^{*}(2), S p\left(M_{+}, M_{-}\right)\right) \subset S p\left(4\left(M_{+}+M_{-}\right), \mathbb{R}\right)$ is realized as

$$
\begin{aligned}
M_{+-} & =\tilde{a}^{R} a_{R}-\tilde{b}^{\mathrm{R}} b_{\mathrm{R}}+\left(M_{+}-M_{-}\right) . \\
K^{R S} & =2 \tilde{a}^{(R} a^{S)}, \quad K^{\mathrm{RS}}=2 \tilde{b}^{(\mathrm{R}} b^{\mathrm{S})}, \quad K^{R \mathrm{R}}=a^{R} b^{\mathrm{R}}+\tilde{a}^{R} \tilde{b}^{\mathrm{R}},
\end{aligned}
$$

where $R, S=1, \cdots, 2 M_{+}$and $\mathrm{R}, \mathrm{S}=1, \ldots, 2 \mathrm{M}_{-}$. As noted above, this is a rather exceptional case, where we still have a compact group in the dual pair, due to the isomorphism $O^{*}(2) \cong U(1)$. At first glance, this case does not seem to suffice the conditions of a reductive dual pair, since the centralizer of $U(1) \cong O^{*}(2)$ in $S p\left(4\left(M_{+}+M_{-}\right), \mathbb{R}\right)$ is not $S p\left(M_{+}, M_{-}\right)$, but $U\left(2 M_{+}, 2 M_{-}\right)$, even though $U(1)$ is the centralizer of $S p\left(M_{+}, M_{-}\right)$. We can distinguish the dual pair $\left(O^{*}(2), S p\left(M_{+}, M_{-}\right)\right)$from $\left(U(1), U\left(2 M_{+}, 2 M_{-}\right)\right)$by enlarging the embedding group $S p\left(4\left(M_{+}+M_{-}\right), \mathbb{R}\right)$ to $S p\left(4\left(M_{+}+M_{-}\right), \mathbb{R}\right) \rtimes \mathbb{Z}_{2}$, where $\mathbb{Z}_{2}$ acts as

$$
\mathcal{P}\left(\begin{array}{c}
a_{R} \\
\tilde{a}^{R} \\
b_{\mathrm{R}} \\
\tilde{b}^{\mathrm{R}}
\end{array}\right) \mathcal{P}^{-1}=\left(\begin{array}{c}
-\tilde{a}_{R} \\
a^{R} \\
-\tilde{b}_{\mathrm{R}} \\
b^{\mathrm{R}}
\end{array}\right),
$$


(see the relevant discussion in section 2.1). Then, we have the dual pair $\left(O^{*}(2) \rtimes \mathbb{Z}_{2}, S p\left(M_{+}, M_{-}\right)\right) \subset S p\left(4\left(M_{+}+M_{-}\right), \mathbb{R}\right) \rtimes \mathbb{Z}_{2}$, where $S p\left(M_{+}, M_{-}\right)$is the centralizer of $O^{*}(2) \rtimes \mathbb{Z}_{2}$ as $U\left(2 M_{+}, 2 M_{-}\right)$is not invariant under the $\mathbb{Z}_{2}$ action (6.3).

To analyze the relevant representations, we begin with the seesaw duality,

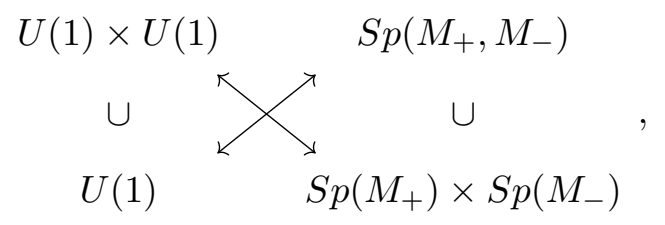

where $U(1) \times U(1)$ is generated by $\tilde{a}^{R} a_{R}+M_{+}$and $-\left(\tilde{b}^{\mathrm{R}} b_{\mathrm{R}}+M_{-}\right)$, whereas $S p\left(M_{+}\right)$and $S p\left(M_{-}\right)$are generated by $K^{R S}$ and $K^{\mathrm{RS}}$. The $\left(U(1) \times U(1), S p\left(M_{+}\right) \times S p\left(M_{-}\right)\right)$duality gives the correspondence,

$$
\left[\ell+M_{+}\right]_{U(1)} \otimes\left[m+M_{-}\right]_{U(1)} \longleftrightarrow \quad[(\ell)]_{S p\left(M_{+}\right)} \otimes[(m)]_{S p\left(M_{-}\right)},
$$

where $\ell, m \in \mathbb{N}$. Any $U(1) \cong O^{*}(2)$ irrep simply consists of an eigenvector of $M_{+-}$,

$$
M_{+-}\left|\Psi_{ \pm k}\right\rangle=\left( \pm k+M_{+}-M_{-}\right)\left|\Psi_{ \pm k}\right\rangle, \quad[k \in \mathbb{N}]
$$

and can therefore result from the tensor product $\left[\ell+M_{+}\right]_{U(1)} \otimes\left[m+M_{-}\right]_{U(1)}$ with

$$
\ell-m= \pm k \text {. }
$$

From the seesaw pair (6.4) and correspondence (6.5), we therefore deduce that the dual $S p\left(M_{+}, M_{-}\right)$representation decomposes into

$$
\bigoplus_{m=0}^{\infty}[(k+m)]_{S p\left(M_{ \pm}\right)} \otimes[(m)]_{S p\left(M_{\mp}\right)}
$$

with Fock states,

$$
\left|\Psi_{+k}\right\rangle=\sum_{m=0}^{\infty} \Psi_{R_{1} \ldots R_{k+m} \mid \mathrm{R}_{1} \ldots \mathrm{R}_{m}} \tilde{a}^{R_{1}} \ldots \tilde{a}^{R_{k+m}} \tilde{b}^{\mathrm{R}_{1}} \ldots \tilde{b}^{\mathrm{R}_{m}}|0\rangle,
$$

and a similar expression for $\left|\Psi_{-k}\right\rangle$ upon exchanging the role of $\tilde{a}$ and $\tilde{b}$. This $S p\left(M_{+}, M_{-}\right)$ representation can be induced from $[(k)]_{S p\left(M_{ \pm}\right)} \otimes[0]_{S p\left(M_{\mp}\right)}$ by the actions of $K^{R \mathrm{R}}$,

$$
\begin{array}{r}
K^{R R}:\left[\left(m_{1}\right)\right]_{S p\left(M_{+}\right)} \otimes\left[\left(m_{2}\right)\right]_{S p\left(M_{-}\right)} \rightarrow\left(\left[\left(m_{1}+1\right)\right]_{S p\left(M_{+}\right)} \otimes\left[\left(m_{2}+1\right)\right]_{S p\left(M_{-}\right)}\right) \\
\oplus\left(\left[\left(m_{1}-1\right)\right]_{S p\left(M_{+}\right)} \otimes\left[\left(m_{2}-1\right)\right]_{S p\left(M_{-}\right)}\right) .
\end{array}
$$

As a consequence, we find the correspondence,

$$
\pi_{S p\left(M_{+}, M_{-}\right)}\left([(k)]_{S p\left(M_{ \pm}\right)} \otimes[(0)]_{S p\left(M_{\mp}\right)}\right) \quad \longleftrightarrow \quad\left[ \pm k+M_{+}-M_{-}\right]_{O^{*}(2) \cong U(1)} \quad[k \in \mathbb{N}],
$$

where $[(0)]_{S p\left(M_{\mp}\right)}$ is the trivial representation of $S p\left(M_{\mp}\right)$. 
For $M_{+}=M_{-}=1$, we have $\widetilde{S O^{+}}(1,4) \cong S p(1,1)$, so that we can interpret the various representations appearing in the previous correspondence as fields around $\mathrm{dS}_{4}$. The irreducible representation $[ \pm 2 s]_{O^{*}(2)}$ with $s \in \frac{1}{2} \mathbb{N}$ corresponds to the $S p(1,1)$ representation induced from $[(s \pm s)]_{S p(1)} \otimes[(s \mp s)]_{S p(1)}$. Due to the isomorphism $S p(1) \times S p(1) \cong \widetilde{S O}(4)$, the latter irrep corresponds to $[(s, \pm s)]_{\overparen{S O}(4)}$, and we find the correspondence,

$$
[ \pm 2 s]_{O^{*}(2)} \longleftrightarrow \quad \pi_{\widetilde{S O}^{+}(1,4)}\left([(s, \pm s)]_{\widetilde{S O}(4)}\right)
$$

The $\widetilde{S O^{+}}(1,4)$ representation describes a massless field of helicity $\pm s$ in $\mathrm{dS}_{4}$ [90-92]. In fact, this representation lies in the discrete series: it corresponds to the irrep $\pi_{p, q}^{ \pm}$with $p=q=s$ of [93] and the irrep $D_{\ell \nu}^{ \pm}$with $\ell=s-1$ and $\nu=1$ in [94].

\section{$6.2(S p(2 N, \mathbb{C}), O(1, \mathbb{C}))$}

The groups of the dual pair $(S p(2 N, \mathbb{C}), O(1, \mathbb{C})) \subset S p(4 N, \mathbb{R})$ are generated by

$$
K^{I J}=\frac{1}{2}\left(\tilde{a}^{I} \tilde{a}^{J}+a^{I} a^{J}-2 \tilde{a}^{(I} a^{J)}\right)
$$

and its complex conjugate ${ }^{16}\left(K^{I J}\right)^{*}$ with $I, J=1, \cdots, 2 N$, and the reflection,

$$
\mathcal{R}=\prod_{I=1}^{2 N} \mathcal{R}_{I}, \quad \mathcal{R}\left(\begin{array}{c}
a^{I} \\
\tilde{a}^{I}
\end{array}\right) \mathcal{R}^{-1}=-\left(\begin{array}{c}
a^{I} \\
\tilde{a}^{I}
\end{array}\right) .
$$

Again the centralizer of $O(1, \mathbb{C})$ is not $S p(2 N, \mathbb{C})$ but $S p(4 N, \mathbb{R})$ itself. We can resolve this problem like the previous case of $\left(S p(p, q), O^{*}(2)\right)$ by enlarging the embedding group $S p(4 M, \mathbb{R})$ with the $\mathbb{Z}_{2}$ action,

$$
\mathcal{P}\left(\begin{array}{c}
a^{I} \\
\tilde{a}^{I}
\end{array}\right) \mathcal{P}^{-1}=\left(\begin{array}{c}
-\tilde{a}^{I} \\
a^{I}
\end{array}\right) .
$$

In this way, we get the dual pair $\left(S p(2 N, \mathbb{C}), O(1, \mathbb{C}) \times \mathbb{Z}_{2}\right) \subset S p(4 M, \mathbb{R}) \rtimes \mathbb{Z}_{2}$, and $S p(2 N, \mathbb{C})$ is the centralizer of $O(1, \mathbb{C}) \times \mathbb{Z}_{2}$.

Let us consider the seesaw duality,

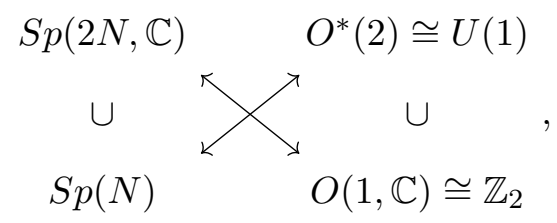

where $S p(N)$ and $O^{*}(2) \cong U(1)$ is realized respectively by $\tilde{a}^{(I} a^{J)}$ and $\tilde{a}^{I} a_{I}+N$. The duality $\left(S p(N), O^{*}(2) \cong U(1)\right)$ gives

$$
[(\ell)]_{S p(N)} \longleftrightarrow \quad[\ell+N]_{U(1)}
$$

\footnotetext{
${ }^{16}$ Recall that $\left(a^{I}\right)^{*}=\eta_{I J} a^{J}$, see (4.24).
} 
where the $U(1)$ irrep can be branched into $O(1, \mathbb{C}) \cong \mathbb{Z}_{2}$ irrep \pm as

$$
\left.[\ell+N]_{U(1)}\right|_{\mathbb{Z}_{2}}=\left[(-1)^{\ell}\right]_{\mathbb{Z}_{2}}
$$

In the end, for a given $O(1, \mathbb{C})$ irrep $[(\bar{n})]_{O(1, \mathbb{C})}=\left[(-1)^{\bar{n}}\right]_{\mathbb{Z}_{2}}$, with $\bar{n}=0$ or 1 , the dual $S p(2 N, \mathbb{C})$ irrep $\pi_{S p(2 N, \mathbb{C})}(\bar{n})$ can be decomposed into

$$
\pi_{S p(2 N, \mathbb{C})}(\bar{n})=\bigoplus_{n=0}^{\infty}[(2 n+\bar{n})]_{S p(N)} \quad[\bar{n}=0,1]
$$

and we find the correspondence,

$$
[(\bar{n})]_{O(1, \mathbb{C})}=\left[(-1)^{\bar{n}}\right]_{\mathbb{Z}_{2}} \quad \longleftrightarrow \quad \pi_{S p(2 N, \mathbb{C})}(\bar{n})
$$

Notice that when $N=1$, we have the isomorphism $S p(2, \mathbb{C}) \cong \widetilde{S O^{+}}(1,3)$, and the irreps $\pi_{S p(2, \mathbb{C})}(0)$ and $\pi_{S p(2, \mathbb{C})}(1)$ correspond to the following irreps in the classification [9] of Harish-Chandra:

$$
\pi_{S p(2, \mathbb{C})}(0):(\mu, j)=\left(i \frac{1}{2}, 0\right), \quad \pi_{S p(2, \mathbb{C})}(1):(\mu, j)=\left(0, \frac{1}{2}\right) .
$$

They sit in the complementary and the principal series, describing respectively a conformally coupled scalar and spinor in $\mathrm{dS}_{3}$. Their tensor product will be considered in the next section, where we analyze the duality $(O(2, \mathbb{C}), S p(2 N, \mathbb{C}))$. These representations are also the relevant ones in Majorana's infinite component spinor equation [12].

\section{Representations of the simplest non-compact dual pairs}

When both of the groups in dual pairs are non-compact, their representations have very different features compared to the case where at least one member is compact. In the latter case, we have seen that the representation space of $G$ or $G^{\prime}$ is spanned by states in the Fock space with a certain excitation number, or in other words, a state is produced by the action of a homogeneous polynomial of the creation operators on the vacuum state. However, in the case of non-compact dual pairs, none of the vectors in the representation space are realized as such polynomials. They instead consists of infinite linear combinations of excitation states, i.e. they are a certain kind of coherent states, and the norms of these states are divergent implying the underlying representations are tempered ones.

\section{$7.1(G L(N, \mathbb{R}), G L(1, \mathbb{R}))$ and $(G L(N, \mathbb{R}), G L(M, \mathbb{R}))$}

The groups of the dual pair $(G L(N, \mathbb{R}), G L(1, \mathbb{R}))$ are respectively generated by

$$
X_{B}^{A}=\frac{1}{2}\left(\tilde{a}^{A} a_{B}-\tilde{a}^{B} a_{A}+\tilde{a}^{A} \tilde{a}^{B}-a_{A} a_{B}\right),
$$

and

$$
Z=\frac{1}{2}\left(\tilde{a}^{A} \tilde{a}^{A}-a_{A} a_{A}\right)
$$


where $A, B=1, \ldots, N$. For the analysis of the representations associated with this duality, let us consider the seesaw pair,

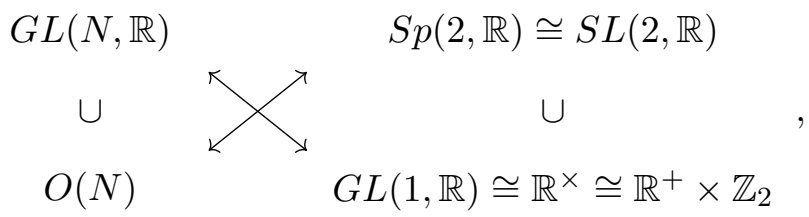

where the maximal compact subgroup $O(N)$ is generated by $X_{B}^{A}-X^{B}{ }_{A}=\tilde{a}^{A} a_{B}-\tilde{a}^{B} a_{A}$ and its dual $S p(2, \mathbb{R}) \cong S L(2, \mathbb{R})$ is generated by

$$
H=\tilde{a}^{A} a_{A}+\frac{N}{2}, \quad E=\frac{1}{\sqrt{2}} \tilde{a}^{A} \tilde{a}^{A}, \quad F=-\frac{1}{\sqrt{2}} a_{A} a_{A},
$$

with the standard Lie bracket (5.68). We begin with the correspondence,

$$
[(\ell)]_{O(N)} \longleftrightarrow \mathcal{D}_{S L(2, \mathbb{R})}\left(\ell+\frac{N}{2}\right)
$$

and consider the restriction $S L(2, \mathbb{R}) \downarrow \mathbb{R}^{+} \times \mathbb{Z}_{2}$ where the $\mathbb{R}^{+}$factor is generated by

$$
Z=\frac{1}{\sqrt{2}}(E+F)
$$

and the $\mathbb{Z}_{2}$ is the reflection group with the reflection element $\mathcal{R}$ acting as

$$
\mathcal{R}\left(\begin{array}{c}
a_{A} \\
\tilde{a}^{A}
\end{array}\right) \mathcal{R}^{-1}=-\left(\begin{array}{c}
a_{A} \\
\tilde{a}^{A}
\end{array}\right) .
$$

The restriction of $S L(2, \mathbb{R})$ to the $\mathbb{Z}_{2}$ determines the parity of $\ell$. The restriction of $S L(2, \mathbb{R})$ to the $\mathbb{R}^{+}$part amounts to finding the spectrum of $Z$ in the positive discrete series representation $\mathcal{D}_{S L(2, \mathbb{R})}$. To tell the conclusion first, the spectrum is the entire set of pure imaginary numbers (as $Z$ is anti-Hermitian), and any $Z$-eigenstate is an infinite linear combinations of $H$-eigenstates, namely a coherent state. As is usual for a coherent state, $Z$-eigenstates in $\mathcal{W}$ do not have finite norms and can be considered only as tempered distributions. More detailed discussions on this point will be provided below. To summarize, for a fixed eigenvalue $i \zeta$ of the $Z$ generator of $\mathbb{R}^{+}$with $\zeta \in \mathbb{R}$ and the sign \pm for $\mathbb{Z}_{2}$, the dual $G L(N, \mathbb{R})$ representation consists of all even/odd $O(N)$ tensors:

$$
\pi_{G L(N, \mathbb{R})}(\zeta, \bar{n}):=\bigoplus_{m=0}^{\infty}[(2 m+\bar{n})]_{O(N)} \quad \longleftrightarrow \quad[\zeta, \bar{n}]_{\mathbb{R}^{\times}}=[\zeta]_{\mathbb{R}^{+}} \otimes\left[(-1)^{\bar{n}}\right]_{\mathbb{Z}_{2}}
$$

Note that the representation space of $G L(N, \mathbb{R})$ does not depend on $\zeta$, but only on $\bar{n}$ (which is 0 or 1$)$. The $\zeta$ dependence can be seen only from the actions of $G L(N, \mathbb{R})$. This is a generic feature of a principal (or complementary) series representation in contrast to the discrete series ones that appeared for the dual pairs involving at least one compact group.

Let us find the explicit $G L(N, \mathbb{R})$ action by solving the $G L(1, \mathbb{R})$ conditions,

$$
Z\left|\Psi_{\zeta, \bar{n}}\right\rangle=i \zeta\left|\Psi_{\zeta, \bar{n}}\right\rangle, \quad \mathcal{R}\left|\Psi_{\zeta, \bar{n}}\right\rangle=(-1)^{\bar{n}}\left|\Psi_{\zeta, \bar{n}}\right\rangle
$$


According to (7.5), a vector in the representation $[(\ell)]_{O(N)} \otimes \mathcal{D}_{S L(2, \mathbb{R})}\left(\ell+\frac{N}{2}\right)$,

$$
A_{1} \quad \cdots \quad A A_{O(N)} \otimes\left|2 n+\ell+\frac{N}{2}\right\rangle \text {, }
$$

is realized by the following state in $\mathcal{W}$,

$$
\left(\tilde{a}^{B} \tilde{a}^{B}\right)^{n} \tilde{a}^{\left\{A_{1}\right.} \ldots \tilde{a}^{\left.A_{\ell}\right\}}|0\rangle
$$

up to a normalization constant. Here, $\left\{A_{1} \cdots A_{\ell}\right\}$ denotes the traceless part of the symmetrization $\left(A_{1} \cdots A_{\ell}\right)$. In order to solve the conditions (7.9), we consider an infinite linear combination of Fock states, namely a coherent state,

$$
\left|T_{\zeta}^{A_{1} \cdots A_{\ell}}\right\rangle=f_{\zeta, \ell}\left(\frac{1}{2} \tilde{a}^{B} \tilde{a}^{B}\right) \tilde{a}^{\left\{A_{1}\right.} \cdots \tilde{a}^{\left.A_{\ell}\right\}}|0\rangle, \quad f_{\zeta, \ell}(z)=\sum_{n=0}^{\infty} c_{n}^{\zeta, \ell} z^{n},
$$

corresponding to an infinite linear combination of $H$-eigenstates,

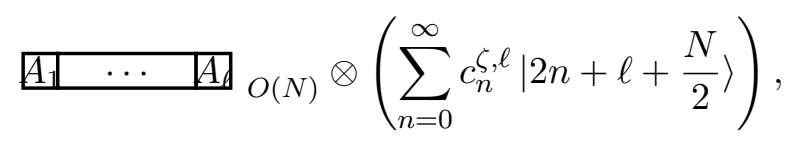

and ask them to satisfy

$$
Z\left|T_{\zeta}^{A_{1} \cdots A_{\ell}}\right\rangle=i \zeta\left|T_{\zeta}^{A_{1} \cdots A_{\ell}}\right\rangle, \quad \mathcal{R}\left|T_{\zeta}^{A_{1} \cdots A_{\ell}}\right\rangle=(-1)^{\ell}\left|T_{\zeta}^{A_{1} \cdots A_{\ell}}\right\rangle
$$

As a consequence, a general state $\left|\Psi_{\zeta, \bar{n}}\right\rangle$ satisfying (7.9) will be given as a linear combination,

$$
\left|\Psi_{\zeta, \bar{n}}\right\rangle=\sum_{n=0}^{\infty} \Psi_{A_{1} \cdots A_{2 n+\bar{n}}}\left|T_{\zeta}^{A_{1} \cdots A_{2 n+\bar{n}}}\right\rangle .
$$

The $Z$-eigenstate condition (7.14) reads

$$
\frac{1}{2}\left(\tilde{a}^{B} \tilde{a}^{B}-a_{B} a_{B}\right) f_{\zeta, \ell}\left(\frac{1}{2} \tilde{a}^{C} \tilde{a}^{C}\right) \tilde{a}^{\left\{A_{1}\right.} \cdots \tilde{a}^{\left.A_{\ell}\right\}}|0\rangle=i \zeta f_{\zeta, \ell}\left(\frac{1}{2} \tilde{a}^{C} \tilde{a}^{C}\right) \tilde{a}^{\left\{A_{1}\right.} \cdots \tilde{a}^{\left.A_{\ell}\right\}}|0\rangle .
$$

The above can be translated into a differential equation ${ }^{17}$ for $h_{\zeta, \ell+\frac{N}{2}}(z)=f_{\zeta, \ell}(z)$,

$$
\left(z \partial_{z}^{2}+\alpha \partial_{z}-z+i \zeta\right) h_{\zeta, \alpha}(z)=0
$$

\footnotetext{
${ }^{17}$ One may also try to solve the $Z$-eigenstate condition starting from a generic state,
}

$$
\left|\Psi_{\zeta, \bar{n}}\right\rangle=\Psi_{\zeta, \bar{n}}(\tilde{a})|0\rangle
$$

where $\Psi_{\zeta, 0 / 1}(\vec{x})$ is a even/odd scalar function of an $N$ dimensional vector $\vec{x}$. Then the condition of (7.14) reduces to the differential equation,

$$
\left(-\nabla^{2}+\vec{x}^{2}-2 i \zeta\right) \Psi_{\zeta, \bar{n}}(\vec{x})=0
$$

having the form of the time independent Schrödinger equation for $N$-dimensional harmonic oscillator with imaginary energy. Since $\Psi_{\zeta, \bar{n}}(\vec{x})$ is not a wave function with $\mathcal{L}^{2}$ scalar norm but a (coherent) Fock state, the "imaginary energy" does not violate unitarity. 
whose solution can be uniquely determined upon imposing $h_{\zeta, \alpha}(0)=1$ as

$$
\begin{aligned}
h_{\zeta, \alpha}(z) & =e^{-z}{ }_{1} F_{1}\left(\frac{\alpha-i \zeta}{2} ; \alpha ; 2 z\right) \\
& =\frac{\Gamma(\alpha)}{2^{\alpha-1} \Gamma\left(\frac{\alpha-i \zeta}{2}\right) \Gamma\left(\frac{\alpha+i \zeta}{2}\right)} \int_{-1}^{1} d t e^{t z}(1+t)^{\frac{\alpha-i \zeta}{2}-1}(1-t)^{\frac{\alpha+i \zeta}{2}-1}
\end{aligned}
$$

Note that the above function has the symmetry,

$$
h_{\zeta, \alpha}(-z)=h_{-\zeta, \alpha}(z)
$$

Let us examine the norm of the states $\left|T_{\zeta}^{A_{1} \cdots A_{\ell}}\right\rangle$ with the simplest example of the scalar states with $\ell=0$ :

$$
\begin{aligned}
\left\langle T_{\zeta^{\prime}} \mid T_{\zeta}\right\rangle= & \left\langle 0\left|h_{\zeta^{\prime}, \frac{N}{2}}^{*}\left(\frac{1}{2} a_{A} a_{A}\right) h_{\zeta, \frac{N}{2}}\left(\frac{1}{2} \tilde{a}_{B} \tilde{a}_{B}\right)\right| 0\right\rangle \\
= & \frac{\Gamma\left(\frac{N}{2}\right)^{2}}{2^{N-2}\left|\Gamma\left(\frac{N}{4}+i \frac{\zeta}{2}\right)\right|^{2}\left|\Gamma\left(\frac{N}{4}+i \frac{\zeta^{\prime}}{2}\right)\right|^{2}} \int_{-1}^{1} d t \int_{-1}^{1} d s(1+t s)^{-\frac{N}{2}} \times \\
& \quad \times(1+t)^{\frac{N}{4}+i \frac{\zeta^{\prime}}{2}-1}(1-t)^{\frac{N}{4}-i \frac{\zeta^{\prime}}{2}-1}(1+s)^{\frac{N}{4}-i \frac{\zeta}{2}-1}(1-s)^{\frac{N}{4}+i \frac{\zeta}{2}-1}
\end{aligned}
$$

where we used

$$
\left\langle 0\left|e^{\frac{t}{2} a_{A} a_{A}} e^{\frac{s}{2} \tilde{a}^{B} \tilde{a}^{B}}\right| 0\right\rangle=(1+t s)^{-\frac{N}{2}} .
$$

The above integral can be simplified by performing the change of variables $t=\tanh \tau$ and $s=\tanh \sigma$, then $u=\sigma+\tau$ and $v=(\sigma-\tau) / 2$. This leads to

$$
\begin{aligned}
\left\langle T_{\zeta^{\prime}} \mid T_{\zeta}\right\rangle & =\frac{\Gamma\left(\frac{N}{2}\right)^{2}}{2^{N-2}\left|\Gamma\left(\frac{N}{4}+i \frac{\zeta}{2}\right)\right|^{2}\left|\Gamma\left(\frac{N}{4}+i \frac{\zeta^{\prime}}{2}\right)\right|^{2}} \int_{-\infty}^{\infty} d u \frac{e^{i\left(\zeta+\zeta^{\prime}\right) \frac{u}{2}}}{\cosh ^{\frac{N}{2}} u} \int_{-\infty}^{\infty} d v e^{i v\left(\zeta-\zeta^{\prime}\right)} \\
& =\frac{2 \pi \Gamma\left(\frac{N}{2}\right)}{2^{\frac{N-2}{2}}\left|\Gamma\left(\frac{N}{4}+i \frac{\zeta}{2}\right)\right|^{2}} \delta\left(\zeta-\zeta^{\prime}\right)
\end{aligned}
$$

The interested readers may consult [95] for additional details where the $N=1$ case was treated explicitly within the context of the $S L(2, \mathbb{R})$ coherent states. The state $\left|T_{\zeta}^{A_{1} \cdots A_{\ell}}\right\rangle$ has a divergent norm in the Fock space, due to the factor $\delta\left(\zeta-\zeta^{\prime}\right)$ appearing in the product of two states $\left|T_{\zeta}^{A_{1} \cdots A_{\ell}}\right\rangle$ and $\left|T_{\zeta^{\prime}}^{A_{1} \cdots A_{\ell^{\prime}}}\right\rangle$. This reflects the fact that states in different representations are orthogonal to one another, but the Dirac distribution blows up when the two $G L(1, \mathbb{R})$ representations coincides. This is a usual property of a coherent state, and from the representation point of view, this means that the relevant representation is a tempered one.

Let us now spell out the $G L(N, \mathbb{R})$ action on the basis $\left|T_{\zeta}^{A_{1} \cdots A_{\ell}}\right\rangle$. First of all, the action of $G L(1, \mathbb{R}) \times O(N) \subset G L(N, \mathbb{R})$ (where $G L(1, \mathbb{R})$ is the center generated by $Z$ ) is clear. The remaining generators are given by the symmetric and traceless part of the $G L(N, \mathbb{R})$ ones,

$$
X_{B\}}^{\{A}=\tilde{a}^{\{A} \tilde{a}^{B\}}-a_{\{A} a_{B\}}
$$


Using the differential equations of $f_{\zeta, \ell}(z)$ and the decomposition,

$$
\tilde{a}^{B} \tilde{a}^{\left\{A_{1}\right.} \cdots \tilde{a}^{\left.A_{n}\right\}}=\tilde{a}^{\{B} \tilde{a}^{A_{1}} \cdots \tilde{a}^{\left.A_{n}\right\}}+\frac{n}{N+2(n-1)} \tilde{a}^{2} \delta^{B\left\{A_{1}\right.} \tilde{a}^{A_{2}} \cdots \tilde{a}^{\left.A_{n}\right\}},
$$

one can derive the action of $X^{\left\{A_{B}\right.}$ on $\left|T^{A_{1} \cdots A_{\ell}} ; \zeta\right\rangle$ as

$$
\begin{aligned}
X_{\left.B_{2}\right\}}^{\left\{B_{1}\right.}\left|T_{\zeta}^{A_{1} \cdots A_{\ell}}\right\rangle= & p_{\ell}\left|T_{\zeta}^{B_{1} B_{2} A_{1} \cdots A_{\ell}}\right\rangle \\
& +q_{\ell} \delta^{\left\{A_{1} \mid\left\{B_{1}\right.\right.}\left|T_{\zeta}^{\left.\left.B_{2}\right\} \mid A_{2} \cdots A_{\ell}\right\}}\right\rangle+r_{\ell} \delta^{\left\{A_{1} \mid\left\{B_{1}\right.\right.} \delta^{\left.B_{2}\right\} \mid A_{2}}\left|T_{\zeta}^{\left.A_{3} \cdots A_{\ell}\right\}}\right\rangle,
\end{aligned}
$$

where the coefficients $p_{\ell}, q_{\ell}$ and $r_{\ell}$ are functions of $\ell, N$ and $\zeta$ :

$$
p_{\ell}=\frac{(N+\ell)(N+2 \ell-4)+4 \zeta}{(N+2 \ell)(N+2 \ell-2)}, \quad q_{\ell}=\frac{2 \ell \zeta}{N+2 \ell}, \quad r_{\ell}=-\frac{\ell^{2}(\ell-1)^{2}}{2(N+2 \ell-2)(N+2 \ell-4)} .
$$

In this way, we find explicit expression of the $G L(N, \mathbb{R})$-representation realized on the space of even or odd symmetric $O(N)$ tensors.

For $N=2$, the restriction of $\pi_{G L(2, \mathbb{R})}(\zeta, \bar{n})$ to $S L(2, \mathbb{R})$ gives the principal series representations, describing scalar/spinor tachyons in $\mathrm{AdS}_{2}$ or massive scalar/spinor in $\mathrm{dS}_{2}$. Together with the discrete series representations $\mathcal{D}_{S L(2, \mathbb{R})}(h+1)$ with $h \in \mathbb{N}$ appearing in the $(U(1,1), U(1))$ pair, these are all oscillator realizations of $S L(2, \mathbb{R})$ in the $\left(G L_{2}, G L_{1}\right)$ dual pairs. Note that the complementary series representations of $S L(2, \mathbb{R})$ do not appear in this oscillator realization.

In fact, the representation we described above becomes much simpler by moving back to the $\omega_{A}$ and $\tilde{\omega}^{A}$ operators with the reality condition (3.7), namely, the Schrödinger realization with real variables $x_{A}$ :

$$
\omega_{A}=\frac{\partial}{\partial x^{A}}, \quad \tilde{\omega}^{A}=x^{A} .
$$

In this realization, the $G L(N, \mathbb{R})$ generators have the form,

$$
X_{B}^{A}=x^{A} \frac{\partial}{\partial x^{B}}+\frac{1}{2} \delta_{B}^{A}, \quad Z=x^{A} \frac{\partial}{\partial x^{A}}+\frac{N}{2} .
$$

The exponentiation of the above gives the action of a group element $g \in G L(N, \mathbb{R})$ as

$$
\left\langle x\left|U_{\mathcal{W}}(g)\right| \Psi_{\zeta, \bar{n}}\right\rangle=|\operatorname{det} g|^{\frac{1}{2}}\left\langle x g \mid \Psi_{\zeta, \bar{n}}\right\rangle \quad[g \in G L(N, \mathbb{R})]
$$

whereas the dual $G L(1, \mathbb{R})=\mathbb{R}^{\times}$acts for an element $a$ as

$$
\left\langle x\left|U_{\mathcal{W}}(h)\right| \Psi_{\zeta, \bar{n}}\right\rangle=h^{\frac{N}{2}}\left\langle h x \mid \Psi_{\zeta, \bar{n}}\right\rangle \quad[h \in G L(1, \mathbb{R})]
$$

In the above, $x g$ should be understood as the right multiplication of the $N \times N$ matrix $g$ on the $N$-vector $x$, i.e. $(x g)^{A}=x^{B} g_{B}{ }^{A}$, where $g_{A}{ }^{B}$ are the components of $g$. The $Z$-eigenstate condition becomes the homogeneity condition,

$$
\left\langle x\left|U_{\mathcal{W}}(h)\right| \Psi_{\zeta, \bar{n}}\right\rangle=\operatorname{sgn}(h)^{\bar{n}}|h|^{i \zeta}\left\langle x \mid \Psi_{\zeta, \bar{n}}\right\rangle .
$$


Using the above condition, we can reduce the representation space to the space of functions on $S^{N-1}$,

$$
\left\langle x \mid \Psi_{\zeta, \bar{n}}\right\rangle=|x|^{-\frac{N}{2}+i \zeta} \psi_{\bar{n}}(\hat{x}), \quad\left[\hat{x}^{A}=x^{A} /|x|\right],
$$

where $\psi_{\bar{n}}(-\hat{x})=(-1)^{\bar{n}} \psi_{\bar{n}}(\hat{x})$. In other words, the representation space is the space of functions on $\mathbb{R} \mathbb{P}^{N-1}$. The scalar product is inherited from that of $\mathcal{L}^{2}\left(\mathbb{R}^{N}\right)$ as

$$
\left\langle\Psi_{\zeta, \bar{n}} \mid \Phi_{\rho, \bar{m}}\right\rangle=\int d^{N} x\left\langle x \mid \Psi_{\zeta, \bar{n}}\right\rangle^{*}\left\langle x \mid \Phi_{\rho, \bar{m}}\right\rangle=2 \pi \delta(\zeta-\rho) \delta_{\bar{n} \bar{m}} \int_{S^{N-1}} d^{N-1} \Omega \psi_{\epsilon}(\hat{x})^{*} \phi_{\epsilon}(\hat{x}),
$$

where the delta function arises from the radial integral,

$$
\int_{0}^{\infty} \frac{d r}{r} r^{-i(\zeta-\rho)}=\int_{-\infty}^{\infty} d t e^{-i(\zeta-\rho) t}=2 \pi \delta(\zeta-\rho) .
$$

Recall that the same delta function appears also in the Fock realization where we use the basis of symmetric $O(N)$ tensors. Let us also recall that the relation between a $\mathcal{L}^{2}\left(\mathbb{R}^{N}\right)$ wave function and a Fock state $|\Psi\rangle=\Psi\left(a^{\dagger}\right)|0\rangle$ is simply

$$
\Psi\left(a^{\dagger}\right)|0\rangle \quad \longleftrightarrow \quad\langle q \mid \Psi\rangle=\Psi\left(\frac{q-\partial_{q}}{\sqrt{2}}\right) e^{-\frac{1}{2} q^{2}},
$$

and hence one can move from one realization to the other using the above expression. The Schrödinger realization of the $G L(N, \mathbb{R})$ representation dual to $G L(1, \mathbb{R})$ is in fact what is referred to as the most degenerate principal series representation (see e.g. [96, 97]).

It is worth noting that the most degenerate principal series representation belongs to a more general class of representations realized as functions on the space of $M \times N$ real matrices $x$ with $M \leq N$, endowed with the right action,

$$
\left\langle x\left|U_{\mathcal{W}}(g)\right| \Psi_{\zeta, \bar{n}}\right\rangle=|\operatorname{det} g|^{\frac{M}{2}}\left\langle x g \mid \Psi_{\zeta, \bar{n}}\right\rangle \quad[g \in G L(N, \mathbb{R})],
$$

and the determinant-homogeneity condition [96, 98],

$$
\left\langle h^{t} x \mid \Psi_{\zeta, \bar{n}}\right\rangle=\operatorname{sgn}(\operatorname{det} h)^{\bar{n}}|\operatorname{det} h|^{-\frac{N}{2}+i \zeta}\left\langle x \mid \Psi_{\zeta, \bar{n}}\right\rangle \quad[h \in G L(M, \mathbb{R})] .
$$

Here, $x g$ and $h^{t} x$ are the products of the $M \times N$ matrix $x$ with respectively the $N \times N$ matrix $g$, and the $M \times M$ matrix $h^{t}$ (where $h^{t}$ denotes the transpose of $h$ ), i.e. $(x g)_{A}^{I}=$ $x_{I}^{B} g_{B}{ }^{A}$ and $\left(h^{t} x\right)_{I}^{A}=\left(h^{t}\right)_{I}^{J} x_{J}^{A}=x_{J}^{A} h_{I}^{J}$. The above representation admits a natural interpretation in the dual pair correspondence $(G L(N, \mathbb{R}), G L(M, \mathbb{R}))$, where $G L(N, \mathbb{R})$ and $G L(M, \mathbb{R})$ are generated by

$$
X_{B}^{A}=x_{I}^{A} \frac{\partial}{\partial x_{I}^{B}}+\frac{M}{2} \delta_{B}^{A} \quad R_{I}^{J}=x_{I}^{A} \frac{\partial}{\partial x_{J}^{A}}+\frac{N}{2} \delta_{I}^{J}, \quad Z=x_{I}^{A} \frac{\partial}{\partial x_{I}^{A}}+\frac{M N}{2} .
$$

Each of $G L(N, \mathbb{R})$ and $G L(M, \mathbb{R})$ are realized, respectively, by right and left actions on the space of $M \times N$ matrices, though the action of $G L(M, \mathbb{R})$ is trivial except for its $G L(1, \mathbb{R})$ subgroup:

$$
\begin{aligned}
\left\langle x\left|U_{\mathcal{W}}(h)\right| \Psi_{\zeta, \bar{n}}\right\rangle & =|\operatorname{det} h|^{\frac{N}{2}}\left\langle h^{t} x \mid \Psi_{\zeta, \bar{n}}\right\rangle \\
& =|\operatorname{det} h|^{i \zeta} \operatorname{sgn}(\operatorname{det} h)^{\bar{n}}\left\langle x \mid \Psi_{\zeta, \bar{n}}\right\rangle \quad[h \in G L(M, \mathbb{R})] .
\end{aligned}
$$


In other words, the degenerate principal series representation of $G L(N, \mathbb{R})$ given by the determinant-homogeneity condition (7.39) is dual to the $G L(M, \mathbb{R})$ representation where $S L(M, \mathbb{R})$ part is trivial. Similarly to the $(G L(N, \mathbb{R}), G L(1, \mathbb{R}))$ case, we can reduce the representation space using the determinant-homogeneity condition (7.39) to the space of functions on the real Grassmann manifold $G r_{M, N}(\mathbb{R})$, isomorphic to

$$
G r_{M, N}(\mathbb{R}) \cong O(N) /(O(M) \times O(N-M)) .
$$

These $G L(N, \mathbb{R})$ representations can be induced by the parabolic subgroup whose Levi factor is $G L(M, \mathbb{R}) \times G L(N-M, \mathbb{R}) \subset G L(N, \mathbb{R})$, with the one-dimensional representation $(\zeta, \bar{n})$ of $G L(M, \mathbb{R})$ and the trivial representation of $G L(N-M, \mathbb{R})$ [96]. The restriction of the degenerate principal series $\pi_{G L(N, \mathbb{R})}(\zeta, \bar{n} ; M)$ to $O(N)$ is [98]

$$
\pi_{G L(N, \mathbb{R})}(\zeta, \bar{n} ; M)=\bigoplus_{h(\ell) \leq M}\left[\left(2 \boldsymbol{\ell}+\bar{n} \mathbf{1}_{M}\right)\right]_{O(N)},
$$

where $\mathbf{1}_{M}=(\underbrace{1, \ldots, 1}_{M})$. In this notation, the most degenerate principal series is $\pi_{G L(N, \mathbb{R})}(\zeta, \bar{n})=\pi_{G L(N, \mathbb{R})}(\zeta, \bar{n} ; 1)$.

Finally, let us briefly mention that the relevance of the above representations for $3 \mathrm{~d}$ scattering amplitude: the scattering amplitude of $N_{+}$incoming and $N_{-}$outgoing $3 \mathrm{~d}$ conformal fields can be viewed as the Poincare singlet in the tensor product of $N_{+}$singletons and $N_{-}$contragredient singletons, (or, $N_{+}$lowest (energy) weight and $N_{-}$highest (energy) weight singletons). The situation can be controlled by the seesaw pair,

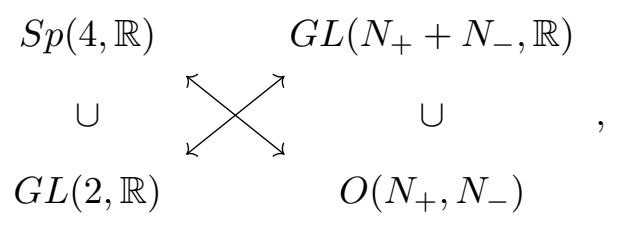

where $G L(2, \mathbb{R})$ decomposes into the dilatation $G L(1, \mathbb{R})$ and the $3 \mathrm{~d}$ Lorentz group $S L(2, \mathbb{R})$, wherein we require the latter to take the trivial representation (so that the amplitude is Lorentz invariant). This is precisely the representation dual to the determinanthomogeneous degenerate principal series representation $\pi_{G L\left(N_{+}+N_{-}, \mathbb{R}\right)}(\zeta, \bar{n})$, which should be restricted to the irrep of $O\left(N_{+}, N_{-}\right)$, whose dual representation is translation invariant. This irrep should be responsible for the form factors of the scattering amplitudes. We shall provide more detailed analysis of this point in a follow-up paper.

\section{$7.2(G L(N, \mathbb{C}), G L(1, \mathbb{C}))$ and $(G L(N, \mathbb{C}), G L(M, \mathbb{C}))$}

The groups of the dual pair $(G L(N, \mathbb{C}), G L(1, \mathbb{C}))$ are respectively generated by

$$
X_{B}^{A}=\frac{1}{2}\left(\tilde{a}^{A} a_{B}-\tilde{b}_{B} b^{A}+\tilde{a}^{A} \tilde{b}_{B}-b^{A} a_{B}\right)
$$

and

$$
Z_{+}=\tilde{a}^{A} \tilde{b}_{A}-a_{A} b^{A}, \quad Z_{-}=\tilde{a}^{A} a_{A}-\tilde{b}_{A} b^{A},
$$


where $A, B=1, \ldots, N$. As $G L(1, \mathbb{C}) \cong \mathbb{R}^{+} \times U(1)$ is Abelian, its representations are simply given by eigenvectors of $Z_{+}$and $Z_{-}$, generating respectively $\mathbb{R}^{+}$and $U(1)$. The decomposition of the $G L(N, \mathbb{C})$ representation under its maximal compact subgroup $U(N)$ is given by the seesaw pair,

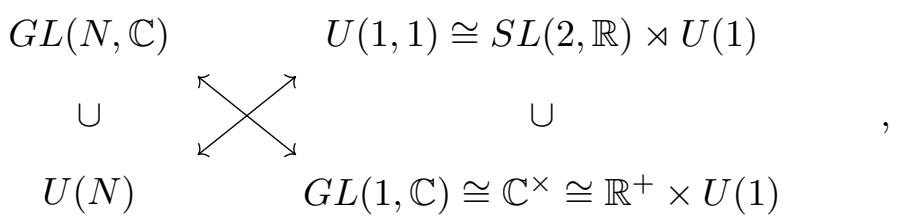

where $U(N)$ is generated by $X_{B}^{A}-\left(X^{B}{ }_{A}\right)^{*}=\tilde{a}^{A} a_{B}-\tilde{b}_{B} b^{A}$ and the $S L(2, \mathbb{R})$ and $U(1)$ subgroups of $U(1,1) \cong S L(2, \mathbb{R}) \rtimes U(1)$ are generated respectively by

$$
H=\tilde{a}^{A} a_{A}+\tilde{b}_{A} b^{A}+N, \quad E=\tilde{a}^{A} \tilde{b}_{A}, \quad F=-a_{A} b^{A},
$$

and

$$
J=\tilde{a}^{A} a_{A}-\tilde{b}_{A} b^{A} .
$$

They are related to the $G L(1, \mathbb{C})$ generators through

$$
Z_{+}=E+F, \quad Z_{-}=J .
$$

Let us consider an irreducible representation of $G L(1, \mathbb{C})$ in the Fock space,

$$
Z_{+}\left|\Psi_{\zeta, m}\right\rangle=i \zeta\left|\Psi_{\zeta, m}\right\rangle, \quad Z_{-}\left|\Psi_{\zeta, m}\right\rangle=m\left|\Psi_{\zeta, m}\right\rangle,
$$

where $\zeta \in \mathbb{R}$ and $m \in \mathbb{Z}$. Henceforth we assume $m \geq 0$ as the $m<0$ case can be treated analogously. According to the correspondence

$$
[(m)]_{U(1)} \otimes \mathcal{D}_{S L(2, \mathbb{R})}(m+2 n+N) \quad \longleftrightarrow \quad[(m+n) \oslash(n)]_{U(N)},
$$

between $U(1,1) \cong S L(2, \mathbb{R}) \rtimes U(1)$ and $U(N)$ irreps and the seesaw pair (7.47), the $G L(N, \mathbb{C})$ representation $\pi_{G L(N, \mathbb{C})}(\zeta, m)$ dual to $[\zeta, m]_{G L(1, \mathbb{C})}$ can be decomposed into

$$
\pi_{G L(N, \mathbb{C})}(\zeta, m):=\bigoplus_{n=0}^{\infty}[(m+n) \oslash(n)]_{U(N)}
$$

As a consequence, any state $\left|\Psi_{\zeta, m}\right\rangle$ in $\pi_{G L(N, \mathbb{C})}(\zeta, m)$ can be written as

$$
\left|\Psi_{\zeta, m}\right\rangle=\sum_{n=0}^{\infty} \Psi_{A_{1} \cdots A_{m+n}}^{B_{1} \cdots B_{n}}\left(f_{\zeta, m, n}\left(\tilde{a}^{C} \tilde{b}_{C}\right) \tilde{a}^{A_{1}} \ldots \tilde{a}^{A_{m+n}} \tilde{b}_{B_{1}} \ldots \tilde{b}_{B_{n}}|0\rangle\right),
$$

where $\delta_{B_{1}}^{A_{1}} \Psi_{A_{1} \cdots A_{m+n}}^{B_{1} \cdots B_{n}}=0$ and the functions $h_{\zeta, m+2 n+N}(z)=f_{\zeta, m, n}(z)$ satisfy the differential equation (7.19), and can be uniquely determined with the condition $f_{\zeta, m, n}(0)=1$. In this way, we find the coherent states carrying a $G L(N, \mathbb{C})$ representation dual to $[\zeta, m]_{G L(1, \mathbb{C})}$. In the case $m<0$, the representation is constructed in the exact same way, except that the $a$ and $b$ oscillators are exchanged. 
Similarly to the $(G L(N, \mathbb{R}), G L(1, \mathbb{R}))$ case, the scalar product of the states $\left|\Psi_{\zeta, m}\right\rangle$ and $\left|\Psi_{\zeta^{\prime}, m^{\prime}}\right\rangle$ is proportional to $\delta\left(\zeta-\zeta^{\prime}\right)$ implying that the relevant representations are tempered ones. They are again known as the most degenerate principal series representations, and we can see this either by directly computing the norm using the precise form of $f_{\zeta, m, n}$ or by switching back to the $\omega_{A}$ and $\tilde{\omega}^{A}$ operators with the reality condition (3.18). The latter amounts to using the Schrödinger realization with complex variables $z_{A}$ :

$$
\omega_{A}=\frac{\partial}{\partial z^{A}}, \quad \omega_{A}^{*}=\frac{\partial}{\partial \bar{z}^{A}}, \quad \tilde{\omega}^{A}=z^{A}, \quad \tilde{\omega}^{A *}=\bar{z}^{A} .
$$

In this realization, the $Z_{ \pm}$generators read

$$
Z_{+}=z^{A} \frac{\partial}{\partial z^{A}}+\bar{z}^{A} \frac{\partial}{\partial \bar{z}^{A}}+N, \quad Z_{-}=z^{A} \frac{\partial}{\partial z^{A}}-\bar{z}^{A} \frac{\partial}{\partial \bar{z}^{A}},
$$

while the $G L(N, \mathbb{C})$ generators are given by

$$
X_{B}^{A}=z^{A} \frac{\partial}{\partial z_{B}}+\frac{1}{2} \delta_{B}^{A},
$$

and their complex conjugate. The associated group action reads

$$
\begin{array}{lll}
\left\langle z\left|U_{\mathcal{W}}(g)\right| \Psi_{\zeta, m}\right\rangle=|\operatorname{det} g|\left\langle z g \mid \Psi_{\zeta, \pm}\right\rangle & & {[g \in G L(N, \mathbb{C})],} \\
\left\langle z\left|U_{\mathcal{W}}(h)\right| \Psi_{\zeta, m}\right\rangle=|h|\left\langle h z \mid \Psi_{\zeta, \pm}\right\rangle & & {[h \in G L(1, \mathbb{C})],}
\end{array}
$$

where $z \in \mathbb{C}^{N}$. The $Z$-eigenstate condition gives the homogeneity condition,

$$
\left\langle z\left|U_{\mathcal{W}}(h)\right| \Psi_{\zeta, m}\right\rangle=h^{\frac{i \zeta+m}{2}} \bar{h}^{\frac{i \zeta-m}{2}}\left\langle z \mid \Psi_{\zeta, m}\right\rangle=|h|^{i \zeta}\left(\frac{h}{|h|}\right)^{m}\left\langle z \mid \Psi_{\zeta, m}\right\rangle .
$$

Using the above condition, we can reduce the representation space to the space of functions on $S^{2 N-1}$,

$$
\left\langle z \mid \Psi_{\zeta, m}\right\rangle=|z|^{-N+i \zeta} \psi_{m}(\hat{z}), \quad\left[|z|=\sqrt{z^{A} \bar{z}^{A}}, \quad \hat{z}^{A}=z^{A} /|z|\right],
$$

where $\psi_{m}$ satisfies the $U(1)$ irrep condition,

$$
\psi_{m}\left(e^{i \phi} \hat{z}\right)=e^{i m \phi} \psi_{m}(\hat{z})
$$

If we mod out this $U(1)$ symmetry, then the representation space is reduced to the space of functions on $\mathbb{C P}^{N-1} \cong S^{2 N-1} / U(1)$, The scalar product is inherited from that of $\mathcal{L}^{2}\left(\mathbb{C}^{N}\right)$, and reads

$$
\begin{aligned}
\left\langle\Psi_{\zeta, m} \mid \Phi_{\rho, n}\right\rangle & =\int d^{N} z d^{N} \bar{z}\left\langle z \mid \Psi_{\zeta, \epsilon}\right\rangle^{*}\left\langle z \mid \Phi_{\rho, \sigma}\right\rangle \\
& =2 \pi \delta(\zeta-\rho) \delta_{m n} \int_{S^{2 N-1}} d^{2 N-1} \Omega \psi_{m}(\hat{z})^{*} \phi_{m}(\hat{z})
\end{aligned}
$$

This representation can be generalized to the space of $M \times N$ complex matrices $z$ with $M \leq N$, endowed with the right action of $G L(N, \mathbb{C})$,

$$
\left\langle z\left|U_{\mathcal{W}}(g)\right| \Psi_{\zeta, m}\right\rangle=|\operatorname{det} g|^{M}\left\langle z g \mid \Psi_{\zeta, m}\right\rangle \quad[g \in G L(N, \mathbb{C})],
$$


and the left action of $G L(M, \mathbb{C})$,

$$
\begin{aligned}
\left\langle z\left|U_{\mathcal{W}}(h)\right| \Psi_{\zeta, m}\right\rangle & =|\operatorname{det} h|^{N}\left\langle h^{t} z \mid \Psi_{\zeta, m}\right\rangle \\
& =|\operatorname{det} h|^{i \zeta}\left(\frac{\operatorname{det} h}{|\operatorname{det} h|}\right)^{m}\left\langle z \mid \Psi_{\zeta, m}\right\rangle \quad[h \in G L(M, \mathbb{C})],
\end{aligned}
$$

which can be interpreted as a determinant-homogeneity condition (see e.g. [98]). The $G L(N, \mathbb{C})$ representation given in $(7.63)$ is dual to the one-dimensional representation $[\zeta, m]_{G L(1, \mathbb{C})} \otimes \mathbf{1}_{S L(M, \mathbb{C})}$ of the dual group $G L(M, \mathbb{C})$ given in $(7.64)$. At the same time, it can be also induced by the parabolic subgroup with Levi factor $G L(M, \mathbb{C}) \times G L(N-M, \mathbb{C}) \subset$ $G L(N, \mathbb{C})$ acting in the aforementioned one-dimensional representation for $G L(M, \mathbb{C})$ and the trivial representation for $G L(N-M, \mathbb{C})$. Using the determinant-homogeneity condition (7.64), we can reduce the representation space to the space of functions on the complex Grassmannian $G r_{M, N}(\mathbb{C})$, isomorphic to

$$
G r_{M, N}(\mathbb{C}) \cong U(N) /(U(M) \times U(N-M))
$$

The restriction of the degenerate principal series $\pi_{G L(N, \mathbb{C})}(\zeta, m, M)$ to $U(N)$ is given in [98] for $M \leq \frac{N}{2}$ as

$$
\pi_{G L(N, \mathbb{C})}(\zeta, m ; M)=\bigoplus_{h(\ell) \leq M}\left[\left(\boldsymbol{\ell}+\frac{|m|+m}{2} \mathbf{1}_{M}\right) \oslash\left(\boldsymbol{\ell}+\frac{|m|-m}{2} \mathbf{1}_{M}\right)\right]_{U(N)}
$$

In this notation, the most degenerate principal series is $\pi_{G L(N, \mathbb{C})}(\zeta, m)=\pi_{G L(N, \mathbb{C})}(\zeta, m ; 1)$.

Like the $(G L(N, \mathbb{R}), G L(2, \mathbb{R}))$ case, the degenerate principal representations appearing in the dual pair $(G L(N, \mathbb{C}), G L(2, \mathbb{C}))$ is relevant for the scattering amplitudes of $4 \mathrm{~d}$ conformal fields. Consider the seesaw pair,

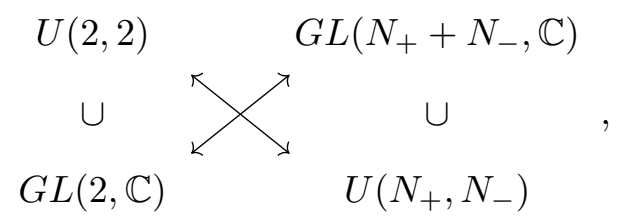

where we can apply exactly the same logic as the seesaw pair for $3 \mathrm{~d}$ amplitudes (7.44). Again, more detailed analysis of this point will be provided in one of our follow-up papers.

$7.3\left(U^{*}(2 N), U^{*}(2)\right)$ and $\left(U^{*}(2 N), U^{*}(2 M)\right)$

The Lie algebra of $U^{*}(2 N)$ is generated by $X^{A}{ }_{B}$ which can be split to the maximal compact subgroup part,

$$
X^{(A B)}=\tilde{a}_{+}^{(A} a^{B)+}+\tilde{a}_{-}^{(A} a^{B)-},
$$

and the rest,

$$
X^{[A B]}=\tilde{a}_{+}^{[A} \tilde{a}_{-}^{B]}-a^{[A+} a^{B]-},
$$


with $A, B=1, \ldots, 2 N$, while the Lie algebra of the dual group $U^{*}(2)$ is generated by

$$
\begin{aligned}
Z & =\tilde{a}_{+}^{A} \tilde{a}_{A-}-a^{A+} a_{A}^{-}, \\
R_{++} & =-J_{+}=-\tilde{a}_{+}^{A} a_{A}^{-}, \quad R_{--}=J_{-}=\tilde{a}_{-}^{A} a_{A}^{+}, \quad R_{+-}=J_{3}=\frac{1}{2}\left(\tilde{a}_{+}^{A} a_{A}^{+}-\tilde{a}_{-}^{A} a_{A}^{-}\right),
\end{aligned}
$$

where $Z$, and $J_{3}, J_{ \pm}$generate respectively $\mathbb{R}^{+}$and $S U(2)$ of $U^{*}(2) \cong \mathbb{R}^{+} \times S U(2)$.

Let us consider the seesaw dual pairs,

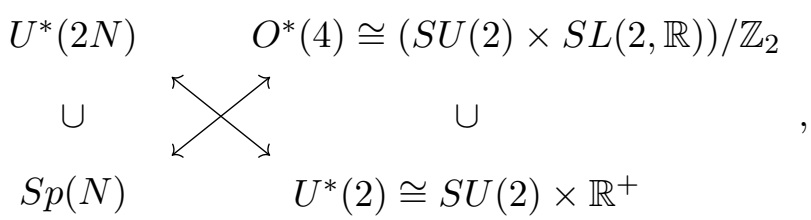

$$
S p(N) \quad U^{*}(2) \cong S U(2) \times \mathbb{R}^{+}
$$

where $O^{*}(4)$ is generated by the $S U(2)$ generators $R_{I J}$, and the $S L(2, \mathbb{R})$ ones given by

$$
H=\tilde{a}_{+}^{A} a_{A}^{+}+\tilde{a}_{-}^{A} a_{A}^{-}+2 N, \quad E=\tilde{a}_{+}^{A} \tilde{a}_{A-}, \quad F=-a^{A+} a_{A}^{-},
$$

with the standard Lie bracket (5.68). We begin with the correspondence,

$$
[(m+n, n)]_{S p(N)} \longleftrightarrow \quad[m]_{S U(2)} \otimes \mathcal{D}_{S L(2, \mathbb{R})}(m+2 n+2 N),
$$

where $n=0$ for $N=1$. Since the two $S U(2)$ 's in $O^{*}(4)$ and $U^{*}(2)$ are the same, the non-trivial part of the restriction $O^{*}(4) \downarrow U^{*}(2)$ is the restriction $S L(2, \mathbb{R}) \downarrow \mathbb{R}$, where $\mathbb{R}$ is embedded in $S L(2, \mathbb{R})$ as

$$
Z=2(E+F) .
$$

Since the spectrum of $Z$ in $\mathcal{D}_{S L(2, \mathbb{R})}(h)$ is the entire set of pure imaginary numbers, we find that the $U^{*}(2 N)$ representation $\pi_{U^{*}(2 N)}(\zeta, m)$, dual to $[\zeta]_{\mathbb{R}^{+}} \otimes[m]_{S U(2)}$, is decomposed as

$$
\pi_{U^{*}(2 N)}(\zeta, m):=\bigoplus_{n=0}^{\infty}[(m+n, n)]_{S p(N)},
$$

and it can be induced from $[(m, 0)]_{S p(N)}$ by the actions of $X^{[A B]}$,

$$
X^{[A B]}:\left[\left(\ell_{1}, \ell_{2}\right)\right]_{S p(N)} \longrightarrow\left[\left(\ell_{1}+1, \ell_{2}+1\right)\right]_{S p(N)} \oplus\left[\left(\ell_{1}-1, \ell_{2}-1\right)\right]_{S p(N)} .
$$

In this way, we find the correspondence,

$$
\pi_{U^{*}(2 N)}(\zeta, m) \quad \longleftrightarrow \quad[\zeta]_{\mathbb{R}^{+}} \otimes[m]_{S U(2)}
$$

Let us consider an irreducible representation of $U^{*}(2)$ in the Fock space,

$$
\begin{aligned}
Z\left|\Psi_{\zeta, m}^{k}\right\rangle & =i \zeta\left|\Psi_{\zeta, m}^{k}\right\rangle, \\
J_{3}\left|\Psi_{\zeta, m}^{k}\right\rangle & =\frac{k}{2}\left|\Psi_{\zeta, m}^{k}\right\rangle, \quad J_{ \pm}\left|\Psi_{\zeta, m}^{k}\right\rangle=\frac{\sqrt{(m \mp k)(m \pm k+2)}}{2}\left|\Psi_{\zeta, m}^{k \pm 2}\right\rangle .
\end{aligned}
$$


The decomposition (7.76) tells us that $\left|\Psi_{\zeta, m}^{m}\right\rangle$ can be written as

$$
\left|\Psi_{\zeta, m}^{m}\right\rangle=\sum_{n=0}^{\infty} \Psi_{A_{1} \ldots A_{m+n}, B_{1} \ldots B_{n}}^{m}\left(f_{\zeta, m, n}\left(\tilde{a}_{+}^{C} \tilde{a}_{C-}\right) \tilde{a}_{+}^{A_{1}} \cdots \tilde{a}_{+}^{A_{m+n}} \tilde{a}_{-}^{B_{1}} \cdots \tilde{a}_{-}^{B_{n}}|0\rangle\right),
$$

where $\Omega^{A_{1} B_{1}} \Psi_{A_{1} \ldots A_{m+n}, B_{1} \ldots B_{n}}^{m}=0$ and the function $h_{\zeta, m+2 n+2 N}(z)=f_{\zeta, m, n}(z)$ satisfies the differential equation as (7.19), and can be uniquely determined with the condition $f_{\zeta, m, n}(0)=1$. Other states $\left|\Psi_{\zeta, m}^{k}\right\rangle$ with $k \neq m$ can be obtained by successive actions of $J_{-}$ on $\left|\Psi_{\zeta, m}^{m}\right\rangle$. Again, the scalar product between the states $\left|\Psi_{\zeta, m}^{k}\right\rangle$ is proportional to $\delta\left(\zeta-\zeta^{\prime}\right)$ and the relevant representations are the tempered ones. They are the most degenerate principal series representations of $U^{*}(2 N)$, similarly to the $G L(N, \mathbb{R})$ and $G L(N, \mathbb{C})$ cases. We can see this by moving back to the $\omega_{A}$ and $\tilde{\omega}^{A}$ operators with the reality condition (3.29). The corresponding Schrödinger realization makes use of $4 N$ complex variables $q_{\epsilon}^{\varepsilon a}$ where $\varepsilon, \epsilon= \pm$ and $a$ takes $N$ values:

$$
\omega_{\varepsilon a}^{\epsilon}=\frac{\partial}{\partial q_{\epsilon}^{\varepsilon a}}, \quad \tilde{\omega}_{\epsilon}^{\varepsilon a}=q_{\epsilon}^{\varepsilon a},
$$

with the reality conditions,

$$
q_{+}^{+a *}=q_{-}^{-a}, \quad q_{-}^{+a *}=-q_{+}^{-a} .
$$

In terms of the above, the generators of $U^{*}(2 N)$ and $U^{*}(2)$ are

$$
X_{\epsilon b}^{\varepsilon a}=q_{\sigma}^{\varepsilon a} \frac{\partial}{\partial q_{\sigma}^{\epsilon b}}+\delta_{b}^{a} \delta_{\epsilon}^{\varepsilon}, \quad R_{\varepsilon}^{\epsilon}=q_{\varepsilon}^{\sigma a} \frac{\partial}{\partial q_{\epsilon}^{\sigma a}}+N \delta_{\varepsilon}^{\epsilon} .
$$

Defining $2 \times 2$ matrix,

$$
q^{a}=\left(\begin{array}{ll}
q_{+}^{+a} & q_{+}^{-a} \\
q_{-}^{+a} & q_{-}^{-a}
\end{array}\right)
$$

the $R_{\varepsilon}{ }^{\epsilon}$ action on it can be written as

$$
p_{\epsilon}^{\varepsilon} R_{\varepsilon}^{\epsilon} q^{a}=\left(\begin{array}{c}
p_{+}^{+} p_{+}^{-} \\
p_{-}^{+}
\end{array} p_{-}^{-}\right)\left(\begin{array}{cc}
q_{+}^{+a} & q_{+}^{-a} \\
q_{-}^{+a} & q_{-}^{-a}
\end{array}\right)+N\left(p_{+}^{+}+p_{-}^{-}\right) q^{a}=p q^{a}+N \operatorname{Tr}(p) q^{a},
$$

Exponentiation of the above reads

$$
\left\langle q\left|U_{\mathcal{W}}(p)\right| \Psi_{\zeta, m}^{k}\right\rangle=|p|^{2 N}\left\langle p q \mid \Psi_{\zeta, m}^{k}\right\rangle \quad[p \in G L(1, \mathbb{H})],
$$

where $p \in U^{*}(2)=G L(1, \mathbb{H})$, and hence can be considered as a non-zero quaternion, and $|p|=\sqrt{\operatorname{det} p}$ as the quaternionic norm. Also the $U^{*}(2 N)$ group action associated to $X^{\varepsilon a}{ }_{\epsilon b}$ can be treated similarly, and it is more natural to view it as $G L(N, \mathbb{H})$ :

$$
\left\langle q\left|U_{\mathcal{W}}(g)\right| \Psi_{\zeta, m}^{k}\right\rangle=(\operatorname{det} g)^{2}\left\langle q g \mid \Psi_{\zeta, m}^{k}\right\rangle \quad[g \in G L(N, \mathbb{H})],
$$

where $q g$ is the right multiplication of the $N \times N$ quaternionic matrix $g$ on the $N$ dimensional quaternionic vector $q$, i.e. $(q g)^{a}=q^{b} g_{b}{ }^{a}$ where $g_{b}{ }^{a}$ are quaternionic components of $g \in G L(N, \mathbb{H})$. Note also that $\operatorname{det} g$ is the determinant of $g$ seen as an element of 
$U^{*}(2 N) \subset G L(2 N, \mathbb{C})$, which is a positive real number, and $\left\langle q \mid \Psi_{\zeta, m}^{k}\right\rangle$ is a square integrable function on $\mathbb{H}^{N}$ taking values in $\mathbb{C}$. The $Z$-eigenstate condition reads

$$
\left\langle q\left|U_{\mathcal{W}}(p)\right| \Psi_{\zeta, m}^{k}\right\rangle=|p|^{i \zeta} D_{m}(\hat{p})_{l}^{k}\left\langle q \mid \Psi_{\zeta, m}^{l}\right\rangle \quad[p \in G L(1, \mathbb{H})]
$$

where $\hat{p}=p /|p|$ is a unit quaternion corresponding to an $S U(2)$ element when viewed as a $2 \times 2$ matrix, and $D_{m}$ is the $(m+1)$-dimensional representation of $S U(2)$. Using the above condition, we can reduce the representation space from the space of functions on $\mathbb{H}^{N} \cong \mathbb{R}^{4 N}$ to the space of functions on $S^{4 N-1}$,

$$
\left\langle q \mid \Psi_{\zeta, m}^{k}\right\rangle=|q|^{-2 N+i \zeta} \psi_{m}^{k}\left(\hat{q}^{a}\right), \quad\left[|q|=\sqrt{q^{a} \bar{q}^{a}}, \quad \hat{q}^{a}=q^{a} /|q|\right],
$$

where $\psi_{m}^{k}$ transforms as

$$
\psi_{m}^{k}\left(\hat{p} \hat{q}^{a}\right)=D_{m}(\hat{p})_{l}^{k} \psi_{m}^{l}\left(\hat{q}^{a}\right) .
$$

If we mod out this $S U(2)$ symmetry, the representation space is reduced to the space of functions on $\mathbb{H} \mathbb{P}^{N-1} \cong S^{4 N-1} / S U(2)$, The scalar product is inherited from that of $\mathcal{L}^{2}\left(\mathbb{H}^{N}\right)$ as

$$
\begin{aligned}
\left\langle\Psi_{\zeta, m}^{k} \mid \Phi_{\rho, n}^{l}\right\rangle & =\int d^{4 N} q\left\langle q \mid \Psi_{\zeta, m}^{k}\right\rangle^{*}\left\langle q \mid \Phi_{\rho, n}^{l}\right\rangle \\
& =2 \pi \delta(\zeta-\rho) \delta_{m n} \delta^{k l} \int_{S^{4 N-1}} d^{4 N-1} \Omega \psi_{m}^{k}(\hat{q})^{*} \phi_{m}^{l}(\hat{q}) .
\end{aligned}
$$

This can be generalized to the $\left(U^{*}(2 N), U^{*}(2 M)\right)$ duality with $M \leq N$, whose representations are realized in the space of $M \times N$ quaternionic matrices $q$, endowed with the right action of $G L(N, \mathbb{H})$,

$$
\left\langle q\left|U_{\mathcal{W}}(g)\right| \Psi_{\zeta}\right\rangle=(\operatorname{det} g)^{2 M}\left\langle q g \mid \Psi_{\zeta}\right\rangle \quad[g \in G L(N, \mathbb{H})]
$$

and the left action of $G L(M, \mathbb{H})$,

$$
\begin{aligned}
\left\langle q\left|U_{\mathcal{W}}(h)\right| \Psi_{\zeta}\right\rangle & =(\operatorname{det} h)^{2 N}\left\langle h^{t} q \mid \Psi_{\zeta}\right\rangle \\
& =(\operatorname{det} h)^{i \zeta}\left\langle q \mid \Psi_{\zeta}\right\rangle \quad[h \in G L(M, \mathbb{H})] .
\end{aligned}
$$

The last equality can be interpreted as a determinant-homogeneity condition (see e.g. [99]). Note here that we do not have the additional label $m$ besides $\zeta$ in contrast with the $(G L(N, \mathbb{H}), G L(1, \mathbb{H}))$ case. This is due to the isomorphism $G L(N, \mathbb{H}) \cong \mathbb{R}^{+} \times S L(N, \mathbb{H})$, where the last factor does not reduce to $S U(2)$ except for $G L(1, \mathbb{H})$. The $G L(N, \mathbb{H})$ representation given in (7.92) is dual to the $[\zeta]_{G L(1, \mathbb{H})} \otimes \mathbf{1}_{S L(M, \mathbb{H})}$ representation of the dual group $G L(M, \mathbb{H})$ given in $(7.93)$. The $G L(N, \mathbb{H})$ representation can be also induced by the parabolic subgroup with Levi factor $G L(M, \mathbb{H}) \times G L(N-M, \mathbb{H}) \subset G L(N, \mathbb{H})$. Using the determinant-homogeneity condition (7.93), we can reduce the representation space to the space of functions on the quaternionic Grassmannian manifold $G r_{M, N}(\mathbb{H})$, isomorphic to

$$
G r_{M, N}(\mathbb{H}) \cong S p(N) /(S p(M) \times S p(N-M))
$$


The restriction of the degenerate principal series $\pi_{G L(N, \mathbb{H})}(\zeta, M)$ to $S p(N)$ is [99]

$$
\pi_{G L(N, \mathbb{H})}(\zeta ; M)=\bigoplus_{\substack{h(\ell) \leq 2 M \\ \ell_{2 i-1}=\ell_{2 i}}}[\ell]_{S p(N)} .
$$

In this notation, the most degenerate principal series $\pi_{U^{*}(2 N)}(\zeta, 0)$ defined in $(7.78)$ corresponds to $\pi_{G L(N, \mathbb{H})}(\zeta ; 1)$.

Analogously to the $(G L(N, \mathbb{R}), G L(2, \mathbb{R}))$ and $(G L(N, \mathbb{C}), G L(2, \mathbb{C}))$ cases, the degenerate principal representations appearing in the dual pair $(G L(N, \mathbb{H}), G L(2, \mathbb{H}))$ can be used for the scattering amplitudes of $6 \mathrm{~d}$ conformal fields. The relevant seesaw pair is

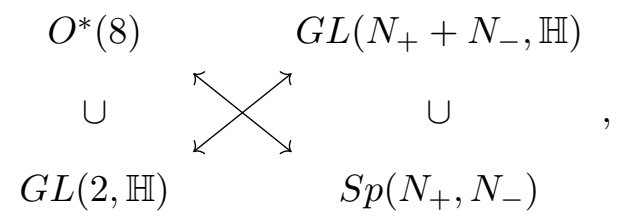

where the same logic as for the seesaw pair of the $3 \mathrm{~d}$ amplitudes (7.44) can be applied. Again, we reserve more detailed analysis of this point for a follow-up paper.

$7.4(S p(2 N, \mathbb{R}), O(1,1))$ and $(S p(2 N, \mathbb{R}), O(M, M))$

The dual pair $(S p(2 N, \mathbb{R}), O(1,1))$ is realized as

$$
\begin{aligned}
K^{+i+j} & =a^{i} a^{j}-\tilde{b}^{i} \tilde{b}^{j}, \quad K^{-i-j}=\tilde{a}_{i} \tilde{a}_{j}-b_{i} b_{j}, \quad K^{+i-j}=\tilde{a}_{j} a^{i}-\tilde{b}^{i} b_{j}, \\
M_{\bullet \bullet} & =\tilde{a}_{i} \tilde{b}^{i}-a^{i} b_{i},
\end{aligned}
$$

where $i, j=1, \ldots, N$. We need to supplement $O(1,1)$ by two reflections $\mathcal{R}_{a}$ and $\mathcal{R}_{b}$ which act as

$$
\mathcal{R}_{a}\left(\begin{array}{c}
a^{i} \\
\tilde{a}_{i}
\end{array}\right) \mathcal{R}_{a}^{-1}=-\left(\begin{array}{c}
a^{i} \\
\tilde{a}_{i}
\end{array}\right), \quad \mathcal{R}_{b}\left(\begin{array}{c}
b_{i} \\
\tilde{b}^{i}
\end{array}\right) \mathcal{R}_{b}^{-1}=-\left(\begin{array}{c}
b_{i} \\
\tilde{b}^{i}
\end{array}\right)
$$

and which form a finite group $\mathbb{Z}_{2}^{a} \times \mathbb{Z}_{2}^{b}$. Let us consider the seesaw pair,

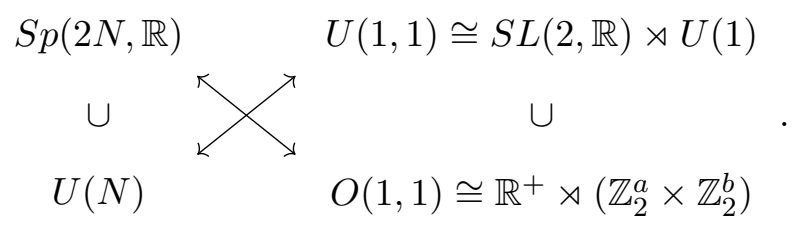

The maximal compact subgroup $U(N)$ of $S p(2 N, \mathbb{R})$ is generated by $K^{+i-j}$, while its dual $U(1,1) \cong S L(2, \mathbb{R}) \rtimes U(1)$ is generated by the $S L(2, \mathbb{R})$ generators,

$$
H=\tilde{a}_{i} a^{i}+\tilde{b}^{i} b_{i}+N, \quad E=\tilde{a}_{i} \tilde{b}^{i}, \quad F=-a^{i} b_{i},
$$

with the usual Lie bracket (5.68), and the diagonal $U(1)$ generator,

$$
Z=\tilde{a}_{i} a^{i}-\tilde{b}^{i} b_{i}
$$


which is also the center $U(1)$ of $U(N) \subset S p(2 N, \mathbb{R})$. Note that $U(1) \subset S L(2, \mathbb{R})$ and the diagonal $U(1)$, generated respectively by $H$ and $Z$, contain the discrete subgroups $\mathbb{Z}_{4}^{+}$and $\mathbb{Z}_{4}^{-}$generated by the elements,

$$
\mathcal{P}_{+}\left(\begin{array}{c}
a^{i} \\
\tilde{a}_{i} \\
b_{i} \\
\tilde{b}^{i}
\end{array}\right) \mathcal{P}_{+}^{-1}=\left(\begin{array}{c}
i a^{i} \\
-i \tilde{a}_{i} \\
i b_{i} \\
-i \tilde{b}^{i}
\end{array}\right), \quad \mathcal{P}_{-}\left(\begin{array}{c}
a^{i} \\
\tilde{a}_{i} \\
b_{i} \\
\tilde{b}^{i}
\end{array}\right) \mathcal{P}_{-}^{-1}=\left(\begin{array}{c}
i a^{i} \\
-i \tilde{a}_{i} \\
-i b_{i} \\
i \tilde{b}^{i}
\end{array}\right) .
$$

They are related to the finite subgroup $\mathbb{Z}_{2}^{a} \times \mathbb{Z}_{2}^{b}$ of $O(1,1)$ as

$$
\mathcal{R}_{a}=\mathcal{P}_{+} \mathcal{P}_{-}, \quad \mathcal{R}_{b}=\mathcal{P}_{+} \mathcal{P}_{-}^{-1} .
$$

The $(U(N), U(1,1))$ duality has the correspondence of the representations,

$$
\begin{aligned}
{[(m) \oslash(n)]_{U(N)} \longleftrightarrow \quad } & \mathcal{D}_{U(1,1)}\left(\left[m+\frac{N}{2}\right]_{U(1)} \otimes\left[n+\frac{N}{2}\right]_{U(1)}\right) \\
& \cong \mathcal{D}_{S L(2, \mathbb{R})}(m+n+N) \otimes[m-n]_{U(1)} .
\end{aligned}
$$

Noting that the isomorphism $O(1,1) \cong \mathbb{R}^{+} \rtimes\left(\mathbb{Z}_{2}^{a} \times \mathbb{Z}_{2}^{b}\right) \cong \mathbb{E} \times \mathbb{Z}_{2}$ where $\mathbb{E}$ is the one dimensional Euclidean group isomorphic to $\mathbb{R}^{+} \rtimes \mathbb{Z}_{2}^{a}$ and $\mathbb{Z}_{2}$ is generated by $\mathcal{R}=\mathcal{R}_{a} \mathcal{R}_{b}$, we find that an irrep of $O(1,1) \cong \mathbb{E} \times \mathbb{Z}_{2}$ is

$$
\pi_{O(1,1)}(\mu, \bar{n}):=[\mu]_{\mathbb{E}} \otimes\left[(-1)^{\bar{n}}\right]_{\mathbb{Z}_{2}} \quad[\mu \geq 0],
$$

where $[\mu]_{\mathbb{E}}$ is one or two dimensional space,

$$
[0]_{\mathbb{E}}=[0]_{\mathbb{R}^{+}}, \quad[\mu]_{\mathbb{E}}=[\mu]_{\mathbb{R}^{+}} \oplus[-\mu]_{\mathbb{R}^{+}} \quad[\mu \geq 0] .
$$

The restriction $U(1,1) \cong S L(2, \mathbb{R}) \rtimes U(1) \downarrow O(1,1) \simeq \mathbb{R}^{+} \rtimes\left(\mathbb{Z}_{2}^{a} \times \mathbb{Z}_{2}^{b}\right)$ amounts to embedding $\mathbb{R}^{+}$in $S L(2, \mathbb{R})$ as

$$
M_{\circ}=E+F,
$$

then supplemented with the restriction to the subgroup $\mathbb{Z}_{2}$ generated by $\mathcal{R}$. The restriction $S L(2, \mathbb{R}) \downarrow \mathbb{R}^{+}$again amounts to finding an $M_{\circ} \bullet$-eigenstate in the positive discrete series representation $\mathcal{D}_{S L(2, \mathbb{R})}(h)$. For any $h$ and $M_{\circ}$-eigenvalue $i \mu$ with $\mu \in \mathbb{R}$, one can find such a (coherent) state. Taking into account the restriction to the $\mathbb{Z}_{2}$ with eigenvalue $\bar{n}$, we find that the representation space of $S p(2 N, \mathbb{R})$ is

$$
\pi_{S p(2 N, \mathbb{R})}(\mu, \bar{n}):=\bigoplus_{\begin{array}{c}
m, n \geq 0 \\
\text { even } m+n+\bar{n}
\end{array}}[(m) \oslash(n)]_{U(N)},
$$

where the $\mu$-dependence does not appear explicitly. For further specification, we can solve the $O(1,1)$ irrep condition,

$$
M_{\circ} \bullet\left|\Psi_{\mu, \bar{n}}^{ \pm}\right\rangle= \pm i \mu\left|\Psi_{\mu, \bar{n}}^{ \pm}\right\rangle, \quad \mathcal{R}_{a}\left|\Psi_{\mu, \bar{n}}^{ \pm}\right\rangle=\left|\Psi_{\mu, \bar{n}}^{\mp}\right\rangle, \quad \mathcal{R}\left|\Psi_{\mu, \bar{n}}^{ \pm}\right\rangle=(-1)^{\bar{n}}\left|\Psi_{\mu, \bar{n}}^{ \pm}\right\rangle
$$


by taking an infinite linear combination of $U(N)$ representation states as

$$
\left|\Psi_{\mu, \bar{n}}^{+}\right\rangle=\sum_{\begin{array}{c}
m, n \geq 0 \\
\text { even } m+n+\bar{n}
\end{array}} \Psi_{i_{1} \cdots i_{m}}^{j_{1} \cdots j_{n}}\left(f_{\mu, m, n}\left(\tilde{a}^{k} \tilde{b}_{k}\right) \tilde{a}^{i_{1}} \cdots \tilde{a}^{i_{m}} \tilde{b}_{j_{1}} \cdots \tilde{b}_{j_{n}}|0\rangle\right),
$$

where the $U(N)$ irrep condition imposes $\delta_{j_{1}}^{i_{1}} \Psi_{i_{1} \cdots i_{m}}^{j_{1} \cdots j_{n}}=0$ and the $M$-eigenstate condition is translated to the differential equation (7.19) for $h_{ \pm \mu, m+n+N}(z)=f_{ \pm \mu, m, n}(z)$, which can be uniquely fixed by $f_{ \pm \mu, m, n}(0)=1$. The other state $\left|\Psi_{\mu, \bar{n}}^{-}\right\rangle$is, by definition, $\mathcal{R}_{a}\left|\Psi_{\mu, \bar{n}}^{+}\right\rangle$.

Note that the $(S p(2 N, \mathbb{R}), O(1,1))$ dual pair can serve also for the tensor product between a metaplectic representation and a contragredient metaplectic representation of $\operatorname{Sp}(2 N, \mathbb{R})$ :

$$
\mathcal{D}_{S p(2 N, \mathbb{R})}\left(\left[(\bar{m}), \frac{1}{2}\right]_{U(N)}\right) \otimes \overline{\mathcal{D}_{S p(2 N, \mathbb{R})}}\left(\left[(\bar{n}), \frac{1}{2}\right]_{U(N)}\right)=\int_{0}^{\infty} d \mu \pi_{S p(2 N, \mathbb{R})}\left(\mu, 1-\delta_{\bar{m} \bar{n}}\right) .
$$

The above can be understood from the seesaw pair,

$$
S p(2 N, \mathbb{R}) \times S p(2 N, \mathbb{R})
$$

where the irrep $[\mu]_{\mathbb{E}} \otimes[\sigma]_{\mathbb{Z}_{2}}$ of $O(1,1)$ branches to the irrep $[(\bar{m})]_{O(1)} \otimes[(\bar{n})]_{O(1)}$ with $(-1)^{\bar{m}+\bar{n}}=\sigma$. Conversely, we can also use the above seesaw diagram to construct the $(S p(2 N, \mathbb{R}), O(1,1))$ representations out of the $(S p(2 N, \mathbb{R}), O(1))$ ones, namely the metaplectic representations. We begin with the tensor product of two metaplectic representations, that is nothing but the Fock space with doubled oscillators (the doubling is from $\left(a_{i}, \tilde{a}^{i}\right)$ to $\left.\left(a_{i}, b^{i}, \tilde{a}^{i}, \tilde{b}_{i}\right)\right)$. The same result (7.111) can be obtained simply imposing the $O(1,1)$ irrep conditions $(7.110)$ on this space. The difference of the construction lies on the viewpoint whether we consider the Fock space as the representation space of $(U(N), U(1,1))$ or the tensor product of the $(S p(2 N, \mathbb{R}), O(1))$ representation space.

For $N=1$, the isomorphism $S p(2, \mathbb{R}) \cong S L(2, \mathbb{R})$ tells that the irrep $\pi_{S p(2, \mathbb{R})}(\mu, \bar{n})$ corresponds to the principal series representation $\pi_{S L(2, \mathbb{R})}\left((-1)^{\bar{n}} \mu\right)$ of $S L(2, \mathbb{R})$. Together with the discrete representations of $(S p(2, \mathbb{R}), O(2))$, they constitute all oscillator realizations of $S L(2, \mathbb{R})$ within all $\left(S p_{2}, O_{2}\right)$ dualities. Again, the complementary series representations do not appear in this construction. Let us summarize here all $S U(2)$ and $S L(2, \mathbb{R})$ oscillator realizations within $S p(4, \mathbb{R})$ :

$$
\begin{array}{cll}
(U(2), U(1)) \&\left(S p(1), O^{*}(2)\right) & \longrightarrow & {[n]_{S U(2)} \quad[n \in \mathbb{N}],} \\
(U(1,1), U(1)) \&(S p(2, \mathbb{R}), O(2)) & \longrightarrow & \mathcal{D}_{S L(2, \mathbb{R})}(h+1) \quad[h \in \mathbb{N}], \\
(G L(2, \mathbb{R}), G L(1, \mathbb{R})) \&(S p(2, \mathbb{R}), O(1,1)) & \longrightarrow & \pi_{S L(2, \mathbb{R})}(\zeta) \quad[\zeta \in \mathbb{R}] . \quad(7.11
\end{array}
$$

When $N=2$, the isomorphism $S p(4, \mathbb{R}) \cong \widetilde{S O}^{+}(2,3)$ suggests that the irreps,

$$
\pi_{S p(4, \mathbb{R})}(\mu, \bar{n})=\bigoplus_{m=-\infty}^{\infty}[2 m+\bar{n}]_{U(1)} \otimes\left(\bigoplus_{n=|m|}^{\infty}[2 n+\bar{n}]_{S U(2)}\right),
$$


may admit a $\mathrm{CFT}_{3}$ or $\mathrm{AdS}_{4}$ interpretation. In fact, it describes the scalar or spinor tachyon in $\mathrm{AdS}_{4}$. This is to be contrasted with the $(S p(4, \mathbb{R}), O(2))$ duality, where we find massless spin $s$ fields. By flipping the signature of the dual orthogonal group, or to put it differently, flipping one of singletons to its contragredient one, the resulting representation has "zero spin" rather than "higher spin". Analogous phenomenon occurs in the $\mathrm{AdS}_{5}$ case, where the relevant dual pairs are $(U(2,2), U(1,1))$ and $(U(2,2), U(2,2))$. Their correspondence will be described in one of the follow-up papers.

The representations that we have constructed are again the most degenerate principal series representation of $S p(2 N, \mathbb{R})$. It will become more transparent if we move to the Schrödinger realization,

$$
y_{A}^{ \pm i}=\frac{1}{\sqrt{2}}\left(x_{A}^{i} \pm \eta_{A B} \frac{\partial}{\partial x_{B}^{i}}\right) .
$$

Let us work first with the dual pair $\left(S p(2 N, \mathbb{R}), O\left(M_{+}, M_{-}\right)\right)$with any $M_{ \pm}$and metric $\eta_{A B}$ of signature $\left(M_{+}, M_{-}\right)$. In this realization, the generators take the analogous form,

$$
M_{A B}=2 x_{[A}^{i} \frac{\partial}{\partial x^{B] i}}, \quad K^{\epsilon i \varepsilon j}=\tilde{K}^{i j}+\epsilon \varepsilon \tilde{K}_{i j}+\frac{\epsilon+\varepsilon}{2} \tilde{K}_{j}^{i},
$$

with

$$
\tilde{K}^{i j}=\frac{1}{2} x_{A}^{i} x^{A j}, \quad \tilde{K}_{i j}=\frac{1}{2} \frac{\partial}{\partial x_{A}^{i}} \frac{\partial}{\partial x^{A j}}, \quad \tilde{K}_{j}^{i}=x_{A}^{i} \frac{\partial}{\partial x_{A}^{j}}+\frac{M_{+}+M_{-}}{2} \delta_{j}^{i} .
$$

Here $\epsilon, \varepsilon= \pm$. The $A, B$ indices above are lowered or raised by $\eta_{A B}$. Now consider the $M_{+}=M_{-}=M$ case with off-diagonal metric $\eta_{A B}$ : setting $A=(+a,-a)$, the metric components are $\eta_{ \pm a \pm b}=0$ and $\eta_{+a-b}=\delta_{a b}$. Then, the generators become

$$
M_{ \pm a \pm b}=x_{ \pm a}^{i} \frac{\partial}{\partial x_{\mp b}^{i}}-x_{ \pm b}^{i} \frac{\partial}{\partial x_{\mp a}^{i}}, \quad M_{+a-b}=x_{+a}^{i} \frac{\partial}{\partial x_{+b}^{i}}-x_{-b}^{i} \frac{\partial}{\partial x_{-a}^{i}},
$$

and

$$
\tilde{K}^{i j}=x_{+a}^{(i} x_{-a}^{j)}, \quad \tilde{K}_{i j}=\frac{\partial}{\partial x_{+a}^{(i}} \frac{\partial}{\partial x_{-a}^{j)}}, \quad \tilde{K}_{j}^{i}=x_{+a}^{i} \frac{\partial}{\partial x_{+a}^{j}}+x_{-a}^{i} \frac{\partial}{\partial x_{-a}^{j}}+M \delta_{j}^{i} .
$$

If we perform a Fourier transformation on the variable $x_{-a}^{i}$, the $S p(2 N, \mathbb{R})$ generators become homogeneous,

$$
\tilde{K}^{i j}=i x_{+a}^{(i} \frac{\partial}{\left.\partial p_{-a j}\right)} \quad \tilde{K}_{i j}=-i p_{-a(i} \frac{\partial}{\partial x_{+b}^{j)}}, \quad \tilde{K}_{j}^{i}=x_{+a}^{i} \frac{\partial}{\partial x_{+a}^{j}}+p_{-a j} \frac{\partial}{\partial p_{-a i}} .
$$

In this way, we find the natural action of $(S p(2 N, \mathbb{R}), O(M, M))$ on the space of $M \times 2 N$ real matrices $\left(x_{+}, p_{-}\right)$or $2 M \times N$ real matrices $\left(\begin{array}{l}x_{+} \\ x_{-}\end{array}\right)\left(x_{ \pm}\right.$and $p_{-}$are $M \times N$ real matrices with components $x_{ \pm a}^{i}$ and $\left.p_{-a i}\right)$ :

$$
\begin{aligned}
\left\langle\left(x_{+}, p_{-}\right)\left|U_{\mathcal{W}}(g)\right| \Psi\right\rangle & =\left\langle\left(x_{+}, p_{-}\right) g \mid \Psi\right\rangle & & {[g \in S p(2 N, \mathbb{R})], } \\
\left\langle\left(\begin{array}{l}
x_{+} \\
x_{-}
\end{array}\right)\left|U_{\mathcal{W}}(h)\right| \Psi\right\rangle & =\left\langle h^{t}\left(\begin{array}{l}
x_{+} \\
x_{-}
\end{array}\right) \mid \Psi\right\rangle & & {[h \in O(M, M)] . }
\end{aligned}
$$


This space is reducible and we can impose the determinant-homogeneity condition,

$$
\left\langle k\left(x_{+}, p_{-}\right) \mid \Psi_{\mu, \bar{n}}^{ \pm}\right\rangle=|\operatorname{det} k|^{ \pm i \mu} \operatorname{sgn}(\operatorname{det} k)^{\bar{n}}\left\langle\left(x_{+}, p_{-}\right) \mid \Psi_{\mu, \bar{n}}^{ \pm}\right\rangle \quad[k \in G L(M, \mathbb{R})],
$$

or equivalently,

$$
\left\langle\left(\begin{array}{cc}
k & 0 \\
0 & \left(k^{-1}\right)^{t}
\end{array}\right)\left(\begin{array}{l}
x_{+} \\
x_{-}
\end{array}\right) \mid \Psi_{\mu, \bar{n}}^{ \pm}\right\rangle=|\operatorname{det} k|^{ \pm i \mu+1} \operatorname{sgn}(\operatorname{det} k)^{\bar{n}}\left\langle\left(\begin{array}{l}
x_{+} \\
x_{-}
\end{array}\right) \mid \Psi_{\mu, \bar{n}}^{ \pm}\right\rangle \quad[k \in G L(M, \mathbb{R})],
$$

to make it irreducible for both of $S p(2 N, \mathbb{R})$ and $O(M, M)$. Note that the additional factor of $|\operatorname{det} k|$ arises from the Jacobian in the Fourier transformation. For $M=1$, one can easily recognize that the condition (7.124) is equivalent to the assignment of the eigenvalue $\pm i \mu$ to the generator $M_{+-}$. This condition coincides in fact with the first one in (7.110) simply because $M_{+-}=M_{\circ}$. The other two conditions can be also easily identified. Therefore, we generalized the representations appearing in $(S p(2 N, \mathbb{R}), O(1,1))$ to the more general case of $(S p(2 N, \mathbb{R}), O(M, M))$. The resulting representations are in fact the degenerate principal series representation for $S p(2 N, \mathbb{R})$ (it becomes the most degenerate one if the dual group is $O(1,1)$ ), and the most degenerate principal series ones for $O(M, M)$. The corresponding parabolic subgroups are the ones with Levi factors $G L(N-M, \mathbb{R}) \times G L(M, \mathbb{R})$ for $S p(2 N, \mathbb{R})$ and $G L(M, \mathbb{R})$ for $O(M, M)$. To put it differently, the representations of $(S p(2 N, \mathbb{R}), O(M, M))$ are obtained by restricting and inducing the $(G L(2 N, \mathbb{R}), G L(M, \mathbb{R}))$ representations, as summarized in the following seesaw diagram:

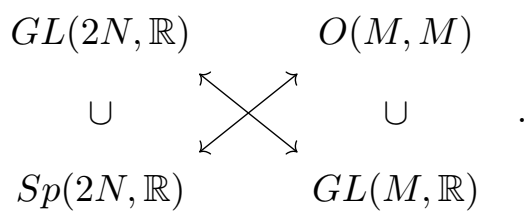

$7.5(S p(2 N, \mathbb{C}), O(2, \mathbb{C}))$ and $(S p(2 N, \mathbb{C}), O(2 M, \mathbb{C}))$

In order to describe $O(2, \mathbb{C})$, it is convenient to define

$$
a_{ \pm}^{I}=\frac{1}{\sqrt{2}}\left(a_{1}^{I} \mp i a_{2}^{I}\right), \quad \tilde{a}_{I}^{ \pm}=\frac{1}{\sqrt{2}}\left(\tilde{a}_{I}^{1} \pm i \tilde{a}_{I}^{2}\right),
$$

where $I=1, \ldots, 2 N$ is the symplectic index. In terms of these oscillators, $O(2, \mathbb{C})$ generators are given by

$$
M_{12}^{+}=i\left(\tilde{a}_{I}^{+} a_{+}^{I}-\tilde{a}_{I}^{-} a_{-}^{I}\right), \quad M_{12}^{-}=i\left(\tilde{a}^{+I} \tilde{a}_{I}^{-}-a_{+}^{I} a_{-I}\right),
$$

with the reflection flipping the sign of $a_{2}^{I}$ and $\tilde{a}_{I}^{2}$,

$$
\mathcal{R}\left(\begin{array}{c}
a_{ \pm}^{I} \\
\tilde{a}_{I}^{ \pm}
\end{array}\right) \mathcal{R}^{-1}=\left(\begin{array}{c}
a_{\mp}^{I} \\
\tilde{a}_{I}^{\mp}
\end{array}\right) .
$$

Recall that the symplectic indices are contracted as in (1.12). The dual group $S p(2 N, \mathbb{C})$ is generated by

$$
\begin{aligned}
& T^{I J}=K^{I J}-\eta_{I K} \eta_{J L}\left(K^{K L}\right)^{*}=-\left(\begin{array}{ll}
\tilde{a}^{+(I} & a_{+}^{J)}+\tilde{a}^{-(I} a_{-}^{J)}
\end{array}\right), \\
& S^{I J}=K^{I J}+\eta_{I K} \eta_{J L}\left(K^{K L}\right)^{*}=i\left(\tilde{a}^{+(I} \tilde{a}^{J)-}-a_{+}^{(I} a_{-}^{J)}\right),
\end{aligned}
$$


where $T^{I J}$ generates the maximal compact subgroup $S p(N)$. The relevant seesaw pair is

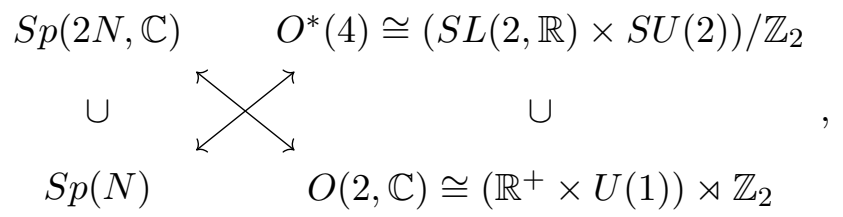

where the correspondence of the $\left(S p(N), O^{*}(4)\right)$ representations is

$$
\left[\left(\ell_{1}, \ell_{2}\right)\right]_{S p(N)} \longleftrightarrow \quad\left[\ell_{1}-\ell_{2}\right]_{S U(2)} \otimes \mathcal{D}_{S L(2, \mathbb{R})}\left(\ell_{1}+\ell_{2}+2 N\right) .
$$

Here, the $S L(2, \mathbb{R})$ and $S U(2)$ of $O^{*}(4)$ are generated by

$$
H=\tilde{a}_{I}^{+} a_{+}^{I}+\tilde{a}_{I}^{-} a_{-}^{I}+2 N, \quad E=\tilde{a}_{I}^{+} \tilde{a}^{-I}, \quad F=-a_{+I} a_{-}^{I},
$$

and

$$
J_{3}=\frac{1}{2}\left(\tilde{a}_{I}^{+} a_{+}^{I}-\tilde{a}_{I}^{-} a_{-}^{I}\right), \quad J_{+}=\tilde{a}_{I}^{+} a_{-}^{I}, \quad J_{-}=\tilde{a}_{I}^{-} a_{+}^{I} .
$$

Let us fix an irrep of $O(2, \mathbb{C})$ as

$$
\pi_{O(2, \mathbb{C})}(\mu, m):=\left([\mu]_{\mathbb{R}^{+}} \otimes[m]_{U(1)}\right) \oplus\left([-\mu]_{\mathbb{R}^{+}} \otimes[-m]_{U(1)}\right) \quad[\mu \geq 0],
$$

which is two dimensional unless $\mu=m=0$. In the latter case, the irrep becomes one dimensional:

$$
\pi_{O(2, \mathbb{C})}(0,0):=[0]_{\mathbb{R}^{+}} \otimes[0]_{U(1)} .
$$

The restriction $O^{*}(4) \downarrow O(2, \mathbb{C})$ amounts to embedding

$$
M_{12}^{+}=2 i J_{3}, \quad M_{12}^{-}=i(E+F),
$$

which determines possible $S p(N)$ irreps as

$$
\ell_{1}-\ell_{2}=|m|+2 k, \quad k \in \mathbb{N}
$$

and hence defines the vector space of the corresponding $S p(2 N, \mathbb{C})$ irrep as

$$
\pi_{S p(2 N, \mathbb{C})}(\mu, m):=\bigoplus_{n, k=0}^{\infty}[(|m|+2 k+n, n)]_{S p(N)} .
$$

For further specifications, we solve the $O(2, \mathbb{C})$ irrep condition,

$$
M_{12}^{+}\left|\Psi_{\mu, m}^{ \pm}\right\rangle= \pm i m\left|\Psi_{\mu, m}^{ \pm}\right\rangle, \quad M_{12}^{-}\left|\Psi_{\mu, m}^{ \pm}\right\rangle= \pm \mu\left|\Psi_{\mu, m}^{ \pm}\right\rangle, \quad \mathcal{R}\left|\Psi_{\mu, m}^{ \pm}\right\rangle=\left|\Psi_{\mu, m}^{\mp}\right\rangle,
$$

as the coherent state,

$$
\left|\Psi_{\mu, m}^{+}\right\rangle=\sum_{n, k=0}^{\infty} \Psi^{I(|m|+2 k+n), J(n)}\left(f_{\mu, m, n, k}\left(\tilde{a}_{K}^{+} \tilde{a}^{-K}\right)\left(\tilde{a}_{I}^{+}\right)^{k+n}\left(\tilde{a}_{I}^{\operatorname{sgn}(m)}\right)^{|m|}\left(\tilde{a}_{I}^{-}\right)^{k}\left(\tilde{a}_{J}^{-}\right)^{n}|0\rangle\right),
$$


where $\Omega_{I J} \Psi^{I\left(\ell_{1}\right), J\left(\ell_{2}\right)}=0$ and $h_{\mu, m+2 n+2 k}(z)=f_{\mu, m, n, k}(z)$ satisfies the differential equation (7.19) and can be fixed uniquely with $f_{\mu, m, n, k}(0)=1$. The other state $\left|\Psi_{\mu, m}^{-}\right\rangle$can be obtained by acting with $\mathcal{R}$ on the above, by definition.

Note that the $(S p(2 N, \mathbb{C}), O(2, \mathbb{C}))$ dual pair can serve also for the tensor product of the irreps $\pi_{S p(2 N, \mathbb{R})}(\bar{n})$ appearing in the $(S p(2 N, \mathbb{C}), O(1, \mathbb{C}))$ dual pair:

$$
\pi_{S p(2 N, \mathbb{C})}(\bar{m}) \otimes \pi_{S p(2 N, \mathbb{C})}(\bar{n})=\int_{0}^{\infty} d \mu \bigoplus_{n=0}^{\infty} \pi_{S p(2 N, \mathbb{C})}\left(\mu, 2 n+1-\delta_{\bar{m} \bar{n}}\right) .
$$

The above can be understood from the seesaw pair,

$$
S p(2 N, \mathbb{C}) \times S p(2 N, \mathbb{C})
$$

where the irrep $[\mu, m]_{O(2, \mathbb{C})}$ branches to the irreps $[(\bar{m})]_{O(1, \mathbb{C})} \otimes[(\bar{n})]_{O(1, \mathbb{C})}$ with $(-1)^{\bar{m}+\bar{n}}=(-1)^{m}$. Conversely, the above seesaw diagram can be used to construct the $(S p(2 N, \mathbb{C}), O(2, \mathbb{C}))$ representations out of the $(S p(2 N, \mathbb{C}), O(1, \mathbb{C}))$ ones: begin with the tensor product Fock space with doubled oscillators (the doubling is from $\left(a^{I}, \tilde{a}_{I}\right)$ to $\left.\left(a_{I}^{ \pm}, \tilde{a}_{ \pm}^{I}\right)\right)$. The same result $(7.141)$ can be obtained simply imposing the $O(2, \mathbb{C})$ irrep conditions (7.140) on this space.

When $N=1$, the $S p(2, \mathbb{C}) \cong \widetilde{S O}^{+}(1,3)$ irreps become simpler since two-row Young diagrams are not allowed for $S p(1) \cong S U(2)$ :

$$
\pi_{S p(2, \mathbb{C})}(\mu, 2 s):=\bigoplus_{k=0}^{\infty}[(|2 s|+2 k)]_{S p(1)} .
$$

These irreps match the entire principal series representations of $\widetilde{S O^{+}}(1,3)$ (see HarishChandra [9]), which describe massive spin $s$ fields in $\mathrm{dS}_{3}$. Note that all these representations, that is, for any mass and any spin, can be obtained by the tensor product of two conformal representations $\pi_{S L(2, \mathbb{C})}(0)$ or $\pi_{S L(2, \mathbb{C})}(1)$, while the latter irreps uplift to the singleton irreps of the conformal group $\widetilde{S O^{+}}(2,3)$, mentioned in section 5.3. Recall that the tensor product of two singletons give the tower of all massless spin $s$ fields in $\mathrm{AdS}_{4}$. Therefore, the direct sum of (7.144) over all masses and spins is the restriction of the aforementioned tower to $\mathrm{dS}_{3}$.

The representations we have constructed is again the most degenerate principal series representation of $S p(2 N, \mathbb{C})$. Let us see this point with the more general case of $(S p(2 N, \mathbb{C}), O(2 M, \mathbb{C}))$ duality with any $M$. We first move to the Schrödinger realization,

$$
y_{A}^{ \pm i}=\frac{1}{\sqrt{2}}\left(z_{A}^{i} \pm \eta_{A B} \frac{\partial}{\partial z_{B}^{i}}\right), \quad y_{A}^{ \pm i *}=\frac{1}{\sqrt{2}}\left(\bar{z}_{A}^{i} \pm \eta_{A B} \frac{\partial}{\partial \bar{z}_{B}^{i}}\right)
$$

where we introduced a metric $\eta_{A B}$ for $O(2 M, \mathbb{C})$, which will also appear in the commutation relation of the $y_{A}^{I}$ operators. Since the group is complex, different signatures of $\eta_{A B}$ are all 
equivalent. In other words, we can move from one to the other by a suitable redefinition of the $y$ operators. For our purpose, it will be convenient to fix the metric $\eta_{A B}$ as the off-diagonal one with non-trivial components $\eta_{+a-b}=\delta_{a b}$ where $A=(+a,-a)$. Then, the $O(2 M, \mathbb{C})$ and $S p(2 N, \mathbb{C})$ generators become

$$
M_{ \pm a \pm b}=z_{ \pm a}^{i} \frac{\partial}{\partial z_{\mp b}^{i}}-z_{ \pm b}^{i} \frac{\partial}{\partial z_{\mp a}^{i}}, \quad M_{+a-b}=z_{+a}^{i} \frac{\partial}{\partial z_{+b}^{i}}-z_{-b}^{i} \frac{\partial}{\partial z_{-a}^{i}}
$$

and

$$
K^{\epsilon i \varepsilon j}=\tilde{K}^{i j}+\epsilon \varepsilon \tilde{K}_{i j}+\frac{\epsilon+\varepsilon}{2} \tilde{K}^{i}{ }_{j}
$$

with

$$
\tilde{K}^{i j}=z_{+a}^{(i} z_{-a}^{j)}, \quad \tilde{K}_{i j}=\frac{\partial}{\partial z_{+a}^{(i}} \frac{\partial}{\partial z_{-a}^{j)}}, \quad \tilde{K}_{j}^{i}=z_{+a}^{i} \frac{\partial}{\partial z_{+a}^{j}}+z_{-a}^{i} \frac{\partial}{\partial z_{-a}^{j}}+M \delta_{j}^{i} .
$$

Here, $\epsilon, \varepsilon= \pm$. The rest of generators are simply the complex conjugate of the above. We can also perform a Fourier transformation on the half of the variable $z_{-a}^{i}$ to render the $S p(2 N, \mathbb{C})$ generators homogeneous:

$$
\tilde{K}^{i j}=i z_{+a}^{(i} \frac{\partial}{\partial w_{-a j)}} \quad \tilde{K}_{i j}=-i w_{-a(i} \frac{\partial}{\partial z_{+b}^{j)}}, \quad \tilde{K}_{j}^{i}=z_{+a}^{i} \frac{\partial}{\partial z_{+a}^{j}}+w_{-a j} \frac{\partial}{\partial w_{-a i}} .
$$

Here, $w_{-a i}$ are the variables in the Fourier space. In the end, we can realize the dual pair $(S p(2 N, \mathbb{C}), O(2 M, \mathbb{C}))$ in the space of functions of $M \times 2 N$ complex matrices $\left(z_{+}, w_{-}\right)$or $\left(z_{+}, z_{-}\right)$:

$$
\begin{aligned}
\left\langle\left(z_{+}, w_{-}\right)\left|U_{\mathcal{W}}(g)\right| \Psi\right\rangle & =\left\langle\left(z_{+}, w_{-}\right) g \mid \Psi\right\rangle & & {[g \in S p(2 N, \mathbb{C})], } \\
\left\langle\left(z_{+}\right)\left|U_{\mathcal{W}}(h)\right| \Psi\right\rangle & =\left\langle h^{t}\left(\begin{array}{l}
z_{+} \\
z_{-}
\end{array}\right) \mid \Psi\right\rangle & & {[h \in O(2 M, \mathbb{C})] . }
\end{aligned}
$$

The determinant-homogeneous condition,

$$
\left\langle k\left(z_{+}, w_{-}\right) \mid \Psi_{\mu, m}^{ \pm}\right\rangle=|\operatorname{det} k|^{ \pm i \mu}\left(\frac{\operatorname{det} k}{|\operatorname{det} k|}\right)^{m}\left\langle\left(z_{+}, w_{-}\right) \mid \Psi_{\mu, m}^{ \pm}\right\rangle \quad[k \in G L(M, \mathbb{C})],
$$

or equivalently,

$$
\left\langle\left(\begin{array}{cc}
k & 0 \\
0 & \left(k^{-1}\right)^{t}
\end{array}\right)\left(\begin{array}{l}
z_{+} \\
z_{-}
\end{array}\right) \mid \Psi_{\mu, m}^{ \pm}\right\rangle=|\operatorname{det} k|^{ \pm i \mu+2}\left(\frac{\operatorname{det} k}{|\operatorname{det} k|}\right)^{m}\left\langle\left(\begin{array}{l}
z_{+} \\
z_{-}
\end{array}\right) \mid \Psi_{\mu, m}^{ \pm}\right\rangle \quad[k \in G L(M, \mathbb{C})],
$$

makes the representation irreducible for both of $S p(2 N, \mathbb{C})$ and $O(2 M, \mathbb{C})$. Note that the additional factor of $|\operatorname{det} k|^{2}$ arises from the Jacobian in the Fourier transformation. The resulting representations correspond to the degenerate principal series representation for $S p(2 N, \mathbb{C})$ and the most degenerate principal series one for $O(2 M, \mathbb{C})$. Their parabolic subgroups are the ones with Levi factors $G L(N-M, \mathbb{C}) \times G L(M, \mathbb{C})$ for $S p(2 N, \mathbb{C})$ and $G L(M, \mathbb{C})$ for $O(2 M, \mathbb{C})$. This parabolic induction can be understood from what is depicted by the seesaw diagram,

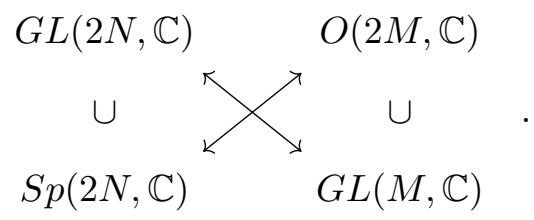




\section{Branching properties}

The representation of $G$ appearing in the dual pair $\left(G, G^{\prime}\right)$, wherein $G^{\prime}$ is the smallest in its family, has several interesting branching properties. In fact, when the group $G$ is the conformal group $S p(4, \mathbb{R}) \cong \widetilde{S O^{+}}(2,3), S U(2,2) \cong \widetilde{S O^{+}}(2,4)$ or $O^{*}(8) \cong \widetilde{S O^{+}}(2,6)$, this representation becomes a conformal field, namely a singleton representation, which possesses a number of special properties (see e.g. [100] for a short review). Therefore, in a sense, we can consider these representations as 'generalized singletons'. In the following, we discuss their branching properties along with a comparison with those of conformal fields.

\subsection{Restriction to maximal compact subgroup}

The $d$-dimensional singleton is the special representation of $\widetilde{S O^{+}}(2, d)$, whose name is a reference to a peculiarity, first derived in [101], namely that its restriction to the maximal compact subgroup $S O(2) \times \widetilde{S O}(d)$ consists in a single direct sum where each irrep appears with multiplicity one.

In fact, all the $G$-representations with the smallest dual group $G^{\prime}$ treated in this paper have such properties. Let us gather the relevant formulas here:

- $(G L(N, \mathbb{R}), G L(1, \mathbb{R}))$ : the $G L(N, \mathbb{R})$ irrep dual to the $G L(1, \mathbb{R}) \cong \mathbb{R}^{+} \otimes \mathbb{Z}_{2}$ irrep $[\zeta]_{\mathbb{R}^{+}} \otimes\left[(-1)^{\bar{n}}\right]_{\mathbb{Z}_{2}}$ decomposes as

$$
\left.\pi_{G L(N, \mathbb{R})}(\zeta, \bar{n})\right|_{O(N)}=\bigoplus_{k=0}^{\infty}[(2 k+\bar{n})]_{O(N)} .
$$

- $(G L(N, \mathbb{C}), G L(1, \mathbb{C}))$ : the $G L(N, \mathbb{C})$ irrep dual to the $G L(1, \mathbb{C}) \cong \mathbb{R}^{+} \otimes U(1)$ irrep $[\zeta]_{\mathbb{R}^{+}} \otimes[n]_{U(1)}$ decomposes as

$$
\left.\pi_{G L(N, \mathbb{C})}(\zeta, n)\right|_{U(N)}=\bigoplus_{k=0}^{\infty}\left[\left(k+\frac{|n|+n}{2}\right) \oslash\left(k+\frac{|n|-n}{2}\right)\right]_{U(N)}
$$

- $\left(U^{*}(2 N), U^{*}(2)\right)$ : the $U^{*}(2 N)$ irrep dual to the $U^{*}(2) \cong \mathbb{R}^{+} \otimes S U(2)$ irrep $[\zeta]_{\mathbb{R}^{+}} \otimes$ $[n]_{S U(2)}$ decomposes as

$$
\left.\pi_{U^{*}(2 N)}(\zeta, n)\right|_{S p(N)}=\bigoplus_{k=0}^{\infty}[(n+k, k)]_{S p(N)} .
$$

- $\left(U\left(N_{+}, N_{-}\right), U(1)\right)$ : the $U\left(N_{+}, N_{-}\right)$irrep dual to the $U(1)$ irrep $[ \pm n]_{U(1)}$ with $n \geq 0$ decomposes as

$$
\begin{gathered}
\left.\mathcal{D}_{U\left(N_{+}, N_{-}\right)}\left(\left[(n), \frac{1}{2}\right]_{U\left(N_{ \pm}\right)} \otimes\left[(0), \frac{1}{2}\right]_{U\left(N_{\mp}\right)}\right)\right|_{U\left(N_{+}\right) \times U\left(N_{-}\right)} \\
=\bigoplus_{k=0}^{\infty}\left[(n+k), \frac{1}{2}\right]_{U\left(N_{ \pm}\right)} \otimes\left[(n+k), \frac{1}{2}\right]_{U\left(N_{\mp}\right)} .
\end{gathered}
$$


- $(S p(2 N, \mathbb{R}), O(1))$ : the $S p(2 N, \mathbb{R})$ irrep dual to the $O(1) \cong \mathbb{Z}_{2}$ irrep $[(\bar{n})]_{O(1)}=$ $\left[(-1)^{\bar{n}}\right]_{\mathbb{Z}_{2}}$ decomposes as

$$
\left.\mathcal{D}_{S p(2 N, \mathbb{R})}\left(\left[(\bar{n}), \frac{1}{2}\right]_{U(N)}\right)\right|_{U(N)}=\bigoplus_{k=0}^{\infty}\left[(2 k+\bar{n}), \frac{1}{2}\right]_{U(N)} .
$$

- $(S p(2 N, \mathbb{C}), O(1, \mathbb{C}))$ : the $S p(2 N, \mathbb{C})$ irrep dual to the $O(1, \mathbb{C}) \cong \mathbb{Z}_{2}$ irrep $[(\bar{n})]_{O(1, \mathbb{C})}=\left[(-1)^{\bar{n}}\right]_{\mathbb{Z}_{2}}$ decomposes as

$$
\left.\pi_{S p(2 N, \mathbb{C})}(\bar{n})\right|_{S p(N)}=\bigoplus_{k=0}^{\infty}[2 k+\bar{n}]_{S p(N)} .
$$

- $\left(O^{*}(2 N), S p(1)\right)$ : the $O^{*}(2 N)$ irrep dual to the $S p(1) \cong S U(2)$ irrep $[n]_{S U(2)}$ decomposes as

$$
\left.\mathcal{D}_{O^{*}(2 N)}\left([(n), 1]_{U(N)}\right)\right|_{U(N)}=\bigoplus_{k=0}^{\infty}[(n+k, k)]_{U(N)} .
$$

- $\left(S p\left(N_{+}, N_{-}\right), O^{*}(2)\right)$ : the $S p\left(N_{+}, N_{-}\right)$irrep dual to the $O^{*}(2) \cong U(1)$ irrep $[ \pm n+$ $\left.N_{+}-N_{-}\right]_{U(1)}$ with $n \geq 0$ decomposes as

$$
\left.\pi_{S p\left(N_{+}, N_{-}\right)}( \pm n)\right|_{S p\left(N_{+}\right) \times S p\left(N_{-}\right)}=\bigoplus_{k=0}^{\infty}[(n+k)]_{S p\left(N_{ \pm}\right)} \otimes[(k)]_{S p\left(N_{\mp}\right)} .
$$

The only cases of $G$-representations with the smallest $G^{\prime}$ that we have not explored are the dual pairs $\left(O\left(N_{+}, N_{-}\right), S p(2, \mathbb{R})\right)$ and $(O(N, \mathbb{C}), S p(2, \mathbb{C}))$, whose representations are not treated in this paper. Let us mention that the scalar singleton of $O(2, d)$ has been explored within the $(O(2, d), S p(2, \mathbb{R}))$ duality in [53] (see also [57]). It is also possible to generalize the above properties to the other branching rules where the maximal compact subgroup $K$ is replaced by a group whose complexification coincides with that of $K$. We shall provide the analysis of these cases together with $\left(O\left(N_{+}, N_{-}\right), S p(2, \mathbb{R})\right)$ and $(O(N, \mathbb{C}), S p(2, \mathbb{C}))$ in our forthcoming paper.

\subsection{Irreducibility under restriction}

Other properties of the $d$-dimensional singletons are that

- they decompose into at most two irreps, when restricted to a $d$-dimensional isometry group, namely the (anti-)de Sitter or Poincaré subgroup (see e.g. [17, 100, 102]),

- and, they are unique, or are one of two possible extensions of a $d$-dimensional isometry irrep to the $d$-dimensional conformal group.

Once again, this property can be investigated using seesaw pairs,

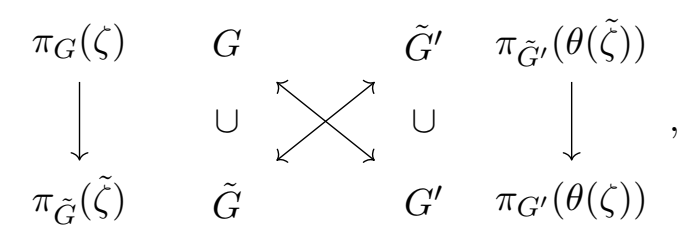


where the conformal group and isometry group can be placed at $G$ and $\tilde{G}$, respectively. In order that a singleton-like irrep $\pi_{G}(\zeta)$ decomposes at most into two irreps $\pi_{\tilde{G}}(\tilde{\zeta})$, on the dual side there should exist at most two irreps $\pi_{\tilde{G}^{\prime}}(\theta(\tilde{\zeta}))$ which can branch to $\pi_{G^{\prime}}(\theta(\zeta))$. Similarly, in order for an irrep $\pi_{\tilde{G}}(\tilde{\zeta})$ to admit an extension to at most two singleton-like irreps of $G$, the restriction of the dual representation $\pi_{\tilde{G}^{\prime}}(\theta(\tilde{\zeta}))$ should contain at most two $G^{\prime}$-irreps. This property is guaranteed, if the dual groups $\tilde{G}^{\prime}$ and $G^{\prime}$ are isomorphic, or isomorphic up to $\mathbb{Z}_{2}$ finite group. The simplest case of $\tilde{G}^{\prime}$ and $G^{\prime}$ is

$$
O(1, \mathbb{C}) \cong O(1)
$$

There are four more such groups among the irreducible ones,

$$
O(2) \supset U(1), \quad O^{*}(2) \cong U(1), \quad O(1,1) \supset G L(1, \mathbb{R}), \quad O(2, \mathbb{C}) \supset G L(1, \mathbb{C}),
$$

which are in fact different real forms of $O_{2} \supseteq G L_{1}$. If one takes into account the reducible pairs, there are two more options,

$$
\tilde{G}^{\prime}=G^{\prime} \times O(1) \supset G^{\prime}, \quad \tilde{G}^{\prime}=G^{\prime} \times O(1, \mathbb{C}) \supset G^{\prime} .
$$

Here, $G^{\prime}$ can be any group suitable for (reducible) dual pairing. In the following, we present the dual groups $G$ and $\tilde{G}$ and their representations for each of the above seven cases.

1. The dual of $O(1, \mathbb{C}) \downarrow O(1)$ is the restriction $S p(4 N, \mathbb{R}) \downarrow S p(2 N, \mathbb{C})$, and we find

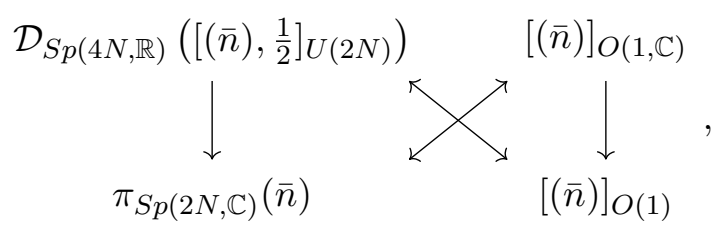

where $\bar{n}=0,1$. The $N=1$ case corresponds to the restriction of the threedimensional singletons to the $\mathrm{dS}_{3}$ subgroup.

2. The dual of $O(2) \downarrow U(1)$ is the restriction of $U(N, N) \downarrow S p(2 N, \mathbb{R})$, and we find

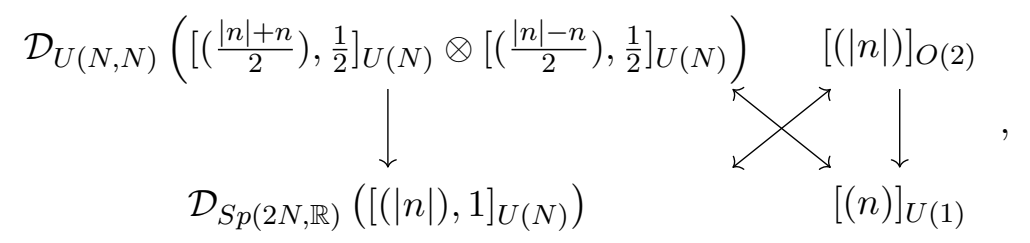

where $n \in \mathbb{Z}$. Notice that for $N \geq 2$, the trivial representation of $U(1)$ can be found in two $O(2)$ representations, $[(0)]_{O(2)}$ and $[(1,1)]_{O(2)}$. As a consequence, the $S p(2 N, \mathbb{R})$ representation dual to this latter $O(2)$ irrep also enters the branching rule, and we find

$$
\begin{aligned}
& \left.\mathcal{D}_{U(N, N)}\left(\left[(0), \frac{1}{2}\right]_{U(N)} \otimes\left[(0), \frac{1}{2}\right]_{U(N)}\right)\right|_{S p(2 N, \mathbb{R})} \\
& =\mathcal{D}_{S p(2 N, \mathbb{R})}\left([(0), 1]_{U(N)}\right) \oplus \mathcal{D}_{S p(2 N, \mathbb{R})}\left([(1,1), 1]_{U(N)}\right) .
\end{aligned}
$$


The $N=2$ case corresponds to the restriction of four-dimensional singletons of helicity $\frac{n}{2}$ to massless fields of spin $\frac{|n|}{2}$ in $\mathrm{AdS}_{4}$ and the restriction of four-dimensional scalar singleton to conformal scalar and pseudo scalar fields in $\mathrm{AdS}_{4}$.

3. The dual of $O^{*}(2) \downarrow U(1)$ is the restriction of $U\left(2 N_{+}, 2 N_{-}\right) \downarrow S p\left(N_{+}, N_{-}\right)$, and we find

$$
\begin{gathered}
\mathcal{D}_{U\left(2 N_{+}, 2 N_{-}\right)}\left(\left[\left(\frac{|n|+n}{2}\right), \frac{1}{2}\right]_{U\left(2 N_{+}\right)} \otimes\left[\left(\frac{|n|-n}{2}\right), \frac{1}{2}\right]_{U\left(2 N_{-}\right)}\right) \\
\pi_{S p\left(N_{+}, N_{-}\right)}\left(\left[\left(\frac{|n|+n}{2}\right)\right]_{S p\left(N_{+}\right)} \otimes\left[\left(\frac{|n|-n}{2}\right)\right]_{S p\left(N_{-}\right)}\right)
\end{gathered}
$$

where $n \in \mathbb{Z}$. The $N_{+}=N_{-}=1$ case corresponds to the restriction of fourdimensional helicity $\frac{n}{2}$ singletons to massless fields of helicity $\frac{n}{2}$ in $\mathrm{dS}_{4}$.

4. The dual of $O(1,1) \downarrow G L(1, \mathbb{R})$ is the restriction $G L(2 N, \mathbb{R}) \downarrow S p(2 N, \mathbb{R})$, and we find

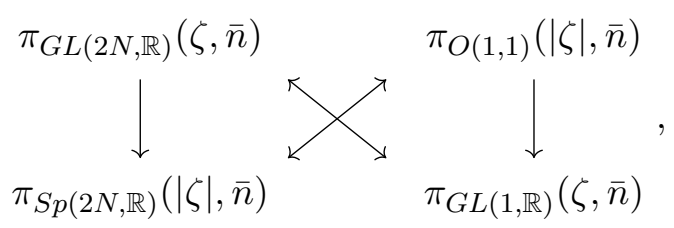

where $\zeta \in \mathbb{R}$ and $\bar{n}=0,1$. The $N=2$ case is to be compared with the $G L(4, \mathbb{R})$ invariant equations for massless fields in $\mathrm{AdS}_{4}$ discussed in [103]. Note, however, that the representations here do not describe massless spin $s$ fields, but scalar or spinor tachyons in $\mathrm{AdS}_{4}$.

5. The dual of $O(2, \mathbb{C}) \downarrow G L(1, \mathbb{C})$ is the restriction $G L(2 N, \mathbb{C}) \downarrow S p(2 N, \mathbb{C})$, and we find

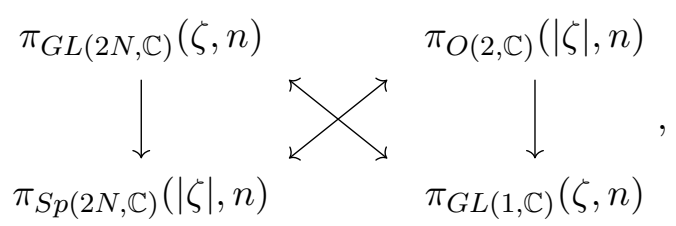

where $\zeta \in \mathbb{R}$ and $n \in \mathbb{Z}$. The $N=1$ case may be interpreted as a lift of the representations to the principal series of $\widetilde{S O^{+}}(1,3)$, which correspond to lifting massive fields of helicity $\frac{n}{2}$ in $\mathrm{dS}_{3}$ to a representation of $G L(2, \mathbb{C})$.

6. Let $G_{N}$ be the family of groups dual to $G^{\prime}$. Then, the dual of $G^{\prime} \times O(1) \downarrow G^{\prime}$ is the restriction $G_{N+M} \downarrow G_{N} \times S p(2 M, \mathbb{R})$, and we find

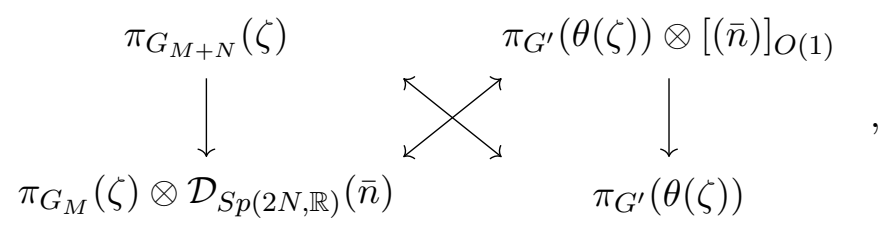


where $\bar{n}=0,1$. The case $M=N=1$ with $G_{2}=\operatorname{Sp}(4, \mathbb{R}), G_{1}=\operatorname{Sp}(2, \mathbb{R})$ and $G^{\prime}=O(1)$ corresponds to the restriction of the three-dimensional singletons to the $\mathrm{AdS}_{3}$ subgroup.

7. The dual of $G^{\prime} \times O(1, \mathbb{C}) \downarrow G^{\prime}$ is the restriction $G_{N+M} \downarrow G_{N} \times S p(2 M, C)$, and we find

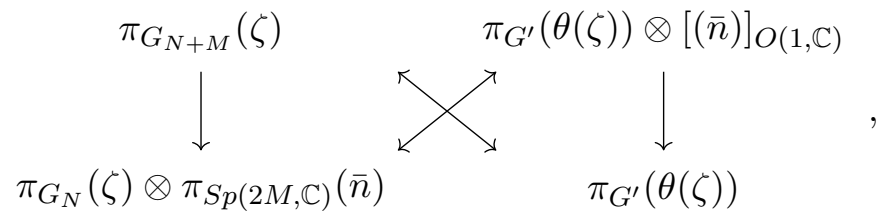

where $\bar{n}=0,1$.

\section{Casimir relations}

One of the consequences of the dual pair correspondence is that the Casimir operators of the two groups in a pair are related [104-107]. Since the Casimir relations can be derived at the level of the metaplectic representation of the embedding symplectic group, it is possible to study them in terms of the previously introduced $\omega$ or $y$ operators without specifying the representations of each group. However, when a specific representation of one group is chosen, the values of its Casimir operators will be fixed. This, in turn, determines the values of the Casimir operators of the dual group, and hence can be used to identify the dual representation. In the following, we derive the relations between Casimir operators for the complex dual pairs, i.e. $\left(G L_{M}, G L_{N}\right)$ and $\left(O_{N}, S p_{2 M}\right)$. The relations of the Casimir operators for real forms of these complex dual pairs can be obtained by imposing the relevant reality conditions.

\subsection{Duality $\left(G L_{M}, G L_{N}\right)$}

We will here derive the relation between the Casimir operators of $G L_{M}$ and $G L_{N}$ in a dual pair. Let us first introduce generating functions for the Casimir operators:

$$
x(t)=\sum_{n=0}^{\infty} t^{n} \mathcal{C}_{n}[\boldsymbol{X}], \quad r(t)=\sum_{n=0}^{\infty} t^{n} \mathcal{C}_{n}[\boldsymbol{R}],
$$

where $\mathcal{C}_{n}[\boldsymbol{X}]$ and $\mathcal{C}_{n}[\boldsymbol{R}]$ are the Casimir operators of order $n$ defined by

$$
\begin{aligned}
\mathcal{C}_{n}[\boldsymbol{X}] & =\operatorname{Tr}\left(\boldsymbol{X}^{n}\right)=X^{A_{1}}{ }_{A_{2}} X^{A_{2}} A_{3} \ldots X^{A_{n}}{ }_{A_{1}}, \\
\mathcal{C}_{n}[\boldsymbol{R}] & =\operatorname{Tr}\left(\boldsymbol{R}^{n}\right)=R_{I_{1}}^{I_{2}} R_{I_{2}}^{I_{3}} \ldots R_{I_{n}}^{I_{1}}
\end{aligned}
$$

Here, $\boldsymbol{X}$ and $\boldsymbol{R}$ stand for matrix-valued operators with components $(\boldsymbol{X})_{A B}=X^{A}{ }_{B}$ and $(\boldsymbol{R})_{I J}=R_{I}{ }^{J}$. It is also convenient to introduce two other generating functions,

$$
\tilde{x}(t)=\sum_{n=0}^{\infty} t^{n} \mathcal{C}_{n}\left[\boldsymbol{X}^{t}\right], \quad \tilde{r}(t)=\sum_{n=0}^{\infty} t^{n} \mathcal{C}_{n}\left[\boldsymbol{R}^{t}\right],
$$


where $\boldsymbol{X}^{t}$ and $\boldsymbol{R}^{t}$ are the "transpose" of $\boldsymbol{X}$ and $\boldsymbol{R}:\left(\boldsymbol{X}^{t}\right)_{A B}=X^{B}{ }_{A}$ and $\left(\boldsymbol{R}^{t}\right)_{I J}=R_{J}{ }^{I}$, and hence the operators $\mathcal{C}_{n}\left[\boldsymbol{X}^{t}\right]$ and $\mathcal{C}_{n}\left[\boldsymbol{R}^{t}\right]$ have the form,

$$
\begin{aligned}
& \mathcal{C}_{n}\left[\boldsymbol{X}^{t}\right]=\operatorname{Tr}\left(\left(\boldsymbol{X}^{t}\right)^{n}\right)=X^{A_{1}}{ }_{A_{n}} X^{A_{2}}{ }_{A_{1}} X^{A_{3}}{ }_{A_{2}} \ldots X^{A_{n}}{ }_{A_{n-1}}, \\
& \mathcal{C}_{n}\left[\boldsymbol{R}^{t}\right]=\operatorname{Tr}\left(\left(\boldsymbol{R}^{t}\right)^{n}\right)=R_{I_{1}}^{{ }^{I_{n}}} R_{I_{2}}^{I_{1}} R_{I_{3}}^{I_{2}} \ldots R_{I_{n}}^{{ }^{I_{n-1}}} .
\end{aligned}
$$

Both $\mathcal{C}_{n}[\boldsymbol{X}]$ and $\mathcal{C}_{n}\left[\boldsymbol{X}^{t}\right]$ are $n$-th order invariants of $G L_{M}$, and their difference shows up starting from $n=3$ :

$$
\mathcal{C}_{3}[\boldsymbol{X}]=X_{B}^{A} X^{B}{ }_{C} X^{C}{ }_{A} \neq X_{B}^{A} X^{C}{ }_{A} X^{B}{ }_{C}=\mathcal{C}_{3}\left[\boldsymbol{X}^{t}\right] .
$$

The freedom in defining the $n$-th order Casimir operators reflects the ambiguity in choosing a basis for the center of the universal enveloping algebra. Below we will also derive a relation between the above two choices, which we will make essential use of to derive a relation between the generating functions in (9.1).

The operator $X^{A}{ }_{B}$ and $R_{I}{ }^{J}$ contain the $(\omega, \tilde{\omega})$ operators in different orders: it is convenient to work with the following order,

$$
Y_{B}^{A}=\tilde{\omega}_{I}^{A} \omega_{B}^{I}, \quad S^{I}=\omega_{A}^{I} \tilde{\omega}_{J}^{A},
$$

which are related to the $X_{B}^{A}$ and $R_{J}^{I}$ by

$$
X_{B}^{A}=Y_{B}^{A}+\frac{N}{2} \delta_{B}^{A}, \quad R_{I}^{J}=S_{I}^{J}-\frac{M}{2} \delta_{I}^{J} .
$$

By introducing generating functions for $\operatorname{Tr}\left[\boldsymbol{Y}^{n}\right]$ and $\operatorname{Tr}\left[\boldsymbol{S}^{n}\right]$ as

$$
y(t)=\sum_{n=0}^{\infty} t^{n} \operatorname{Tr}\left(\boldsymbol{Y}^{n}\right), \quad s(t)=\sum_{n=0}^{\infty} t^{n} \operatorname{Tr}\left(\boldsymbol{S}^{n}\right),
$$

we can find the relation between $x(t)$ and $y(t)$,

$$
x(t)=\operatorname{Tr}\left[(1-t \boldsymbol{X})^{-1}\right]=\frac{1}{1-\frac{N}{2} t} \operatorname{Tr}\left[\left(1-\frac{t}{1-\frac{N}{2} t} \boldsymbol{Y}\right)^{-1}\right]=\frac{y\left(\frac{t}{1-\frac{N}{2} t}\right)}{1-\frac{N}{2} t} .
$$

Similarly, $s(t)$ is related to $\tilde{r}(t)$ as

$$
s(t)=\frac{\tilde{r}\left(\frac{t}{1-\frac{M}{2} t}\right)}{1-\frac{M}{2} t} .
$$

Now, let us relate $y(t)$ and $s(t)$ : in the definition of $\operatorname{Tr}\left(\boldsymbol{Y}^{n}\right)$, we move the $\tilde{\omega}$ operator in the left end to the right end and find the relation,

$$
\operatorname{Tr}\left(\boldsymbol{Y}^{n}\right)=\operatorname{Tr}\left(\boldsymbol{S}^{n}\right)-\sum_{k=0}^{n-1} \operatorname{Tr}\left(\boldsymbol{S}^{k}\right) \operatorname{Tr}\left(\boldsymbol{Y}^{n-1-k}\right) \quad[n \geq 1] .
$$


This relation can be rephrased in terms of the generating functions as

$$
y(t)-M=s(t)-N-t s(t) y(t),
$$

which can be solved as

$$
y(t)=\frac{s(t)+M-N}{1+t s(t)} .
$$

Finally combining the results so far obtained, we find the relation,

$$
x(t)=\frac{\tilde{r}\left(\frac{t}{1-\frac{M+N}{2} t}\right)+(M-N) \frac{1-\frac{M+N}{2} t}{1-\frac{N}{2} t}}{1-\frac{M+N}{2} t+t \cdot \tilde{r}\left(\frac{t}{1-\frac{M+N}{2} t}\right)} .
$$

The formula (9.14) generates the relations between $\mathcal{C}_{n}[\boldsymbol{X}]$ and $\tilde{\mathcal{C}}_{n}[\boldsymbol{R}]$. Next we would like to relate the generating functions $r(t)$ and $\tilde{r}(t)$. This computation is done in appendix D.1 and the result is

$$
\tilde{r}(t)=\frac{r\left(\frac{t}{1+N t}\right)}{1+N t-t \cdot r\left(\frac{t}{1+N t}\right)},
$$

which can be inserted in (9.14) to arrive at

$$
x(t)=\frac{1}{1-\frac{N}{2} t}\left[\frac{1+\frac{N-2 M}{2} t}{1+\frac{N-M}{2} t} r\left(\frac{t}{1+\frac{N-M}{2} t}\right)+M-N\right] .
$$

The inverse relation is given by

$$
r(t)=\frac{1}{1-\frac{M}{2} t}\left[\frac{1+\frac{M-2 N}{2} t}{1+\frac{M-N}{2} t} x\left(\frac{t}{1+\frac{M-N}{2} t}\right)+N-M\right] .
$$

This is the same formula as (9.16) with $x(t) \leftrightarrow r(t)$ and $M \leftrightarrow N$, as expected. The first few relations, generated by $(9.16)$, read

$$
\begin{aligned}
& \mathcal{C}_{1}[\boldsymbol{X}]=\mathcal{C}_{1}[\boldsymbol{R}]=Z \\
& \mathcal{C}_{2}[\boldsymbol{X}]=\mathcal{C}_{2}[\boldsymbol{R}]-\frac{M N(M-N)}{4}, \\
& \mathcal{C}_{3}[\boldsymbol{X}]=\mathcal{C}_{3}[\boldsymbol{R}]+\frac{(M-N)}{8}\left[4 \mathcal{C}_{2}[\boldsymbol{R}]-2(M+N) Z-M^{2} N\right] \\
& \mathcal{C}_{4}[\boldsymbol{X}]=\mathcal{C}_{4}[\boldsymbol{R}]+\frac{(M-N)}{16}\left[16 \mathcal{C}_{3}[\boldsymbol{R}]-8 N \mathcal{C}_{2}[\boldsymbol{R}]-4 M^{2} Z-M N\left(M^{2}-M N+N^{2}\right)\right]
\end{aligned}
$$

A straightforward check shows that our result for the Casimir relations at any order (9.16) reproduce the relations of [107] (Theorem A).

The Casimir operators $\mathcal{C}_{n}[\boldsymbol{R}]$ with $n>N$ are given by the lower order Casimirs, and the relation can be extracted from

$$
\operatorname{det}(1-t \boldsymbol{R})=\exp \left(-\sum_{n=1}^{\infty} \frac{t^{n}}{n} \mathcal{C}_{n}[\boldsymbol{R}]\right)=1-p_{N}(t),
$$


where $p_{N}(t)$ is an order $N$ polynomial with $p_{N}(0)=0$. The coefficients of $p_{N}(t)$ parameterize the independent Casimir operators. By differentiating the above equation, we obtain the relation between $p_{N}(t)$ and the generating function $r(t)$ of $\mathcal{C}_{n}[\boldsymbol{R}]$ :

$$
r(t)=N+\frac{t p_{N}^{\prime}(t)}{1-p_{N}(t)} .
$$

We can put this expression into (9.16) to obtain a rational function whose numerator and denominator have degree $N+1$ and $N+2$, respectively.

One can also solve for $\tilde{r}(t)$ in (9.14) and get the following formula:

$$
\tilde{r}(t)=\frac{x\left(\frac{t}{1+\frac{M+N}{2} t}\right)+(N-M) \frac{1+\frac{M+N}{2} t}{1+\frac{M}{2} t}}{1+\frac{M+N}{2} t-t x\left(\frac{t}{1+\frac{M+N}{2} t}\right)} .
$$

We could do exactly the same computation as when deriving (9.14), but for the $r(t)$ in terms of $\tilde{x}(t)$, and get a similar relation with replaced $M$ and $N$ :

$$
r(t)=\frac{\tilde{x}\left(\frac{t}{1-\frac{M+N}{2} t}\right)+(N-M) \frac{1-\frac{M+N}{2} t}{1-\frac{M}{2} t}}{1-\frac{M+N}{2} t+t \tilde{x}\left(\frac{t}{1-\frac{M+N}{2} t}\right)} .
$$

One may notice that the relations (9.22) and (9.23) are interchanged upon $(t, \tilde{r}(t), x(t)) \leftrightarrow$ $(-t, r(-t), \tilde{x}(-t)): r(-t)$ depends on $\tilde{x}(-t)$ exactly same way as $\tilde{r}(t)$ on $x(t)$. This can be understood in the following way: the difference in the structure of the $r(t)$ and $\tilde{r}(t)$, and correspondingly $x(t)$ and $\tilde{x}(t)$ are given via commutators, which are changing their signs when we change the sign of the generators. On the other hand, changing the signs of the commutators is equivalent to taking the opposite product rule.

\subsection{Duality $\left(O_{N}, S p_{2 M}\right)$}

We finally provide the duality relations between the Casimir operators $\mathcal{C}_{n}[\boldsymbol{M}]=\operatorname{Tr}\left(\boldsymbol{M}^{n}\right)$ and $\mathcal{C}_{n}[\boldsymbol{K}]=\operatorname{Tr}\left(\boldsymbol{K}^{n}\right)$ to all orders in $n$. Compared to the $\left(G L_{M}, G L_{N}\right)$ case of the previous section, the $\left(O_{N}, S p_{2 M}\right)$ case requires more steps of computations, and we leave most details to the appendix D.2. We begin with defining the operators,

$$
N_{B}^{A}=y_{I}^{A} y_{B}^{I}, \quad L_{J}^{I}=y_{A}^{I} y_{J}^{A},
$$

where $y_{I}^{A}$ is defined as

$$
y_{I}^{A}=E^{A B} \Omega_{I J} y_{B}^{J} .
$$

and satisfies

$$
\left[y_{I}^{A}, y_{B}^{J}\right]=\delta_{B}^{A} \delta_{I}^{J} .
$$

The operators $N^{A}{ }_{B}$ and $L^{I}{ }_{J}$ are related to $M^{A}{ }_{B}$ and $K^{I}{ }_{J}$ by ${ }^{18}$

$$
N_{B}^{A}=-M_{B}^{A}+M \delta_{B}^{A}, \quad L_{J}^{I}=-\left(K_{J}^{I}+\frac{N}{2} \delta_{J}^{I}\right),
$$

\footnotetext{
${ }^{18}$ The numbers $N$ and $M$ should not be confused with the generators $N^{A}{ }_{B}$ and $M^{A}{ }_{B}$ in this section.
} 
where $M_{B}^{A}:=E^{A C} M_{C B}$ and $K_{J}^{I}:=K^{I K} \Omega_{K J}$.

By defining generating functions,

$$
\begin{aligned}
m(t) & =\sum_{n=0}^{\infty} t^{n} \mathcal{C}_{n}[\boldsymbol{M}], & k(t) & =\sum_{n=0}^{\infty} t^{n} \mathcal{C}_{n}[\boldsymbol{K}], \\
n(t) & =\sum_{n=0}^{\infty} t^{n} \operatorname{Tr}\left(\boldsymbol{N}^{n}\right), & l(t) & =\sum_{n=0}^{\infty} t^{n} \operatorname{Tr}\left(\boldsymbol{L}^{n}\right),
\end{aligned}
$$

it is possible to derive exact relations between the Casimir operators to all orders through their generating functions. To derive these relations we find it useful to expand the space of generating functions to the following set of two-parameter family of generating functions:

$$
\begin{aligned}
& a(t, u)=\sum_{k, l=0}^{\infty} t^{k} u^{l I}[2 k+1]_{A I}[2 l+1]^{A} \\
& b(t, u)=\sum_{k, l=0}^{\infty} t^{k} u^{l B}[2 k]_{A B}[2 l]^{A}, \quad d(t, u)=\sum_{k, l=0}^{\infty} t^{k} u^{l I}[2 k]_{J}[2 l]^{J},
\end{aligned}
$$

where the brackets are short-hand for

$$
{ }^{\alpha}[n]_{\beta}:=y_{\gamma_{2}}^{\alpha} y_{\gamma_{3}}^{\gamma_{2}} \cdots y_{\beta}^{\gamma_{n}}, \quad{ }_{\alpha}[n]^{\beta}:=y_{\alpha}^{\gamma_{2}} y_{\gamma_{2}}^{\gamma_{3}} \cdots y_{\gamma_{n}}^{\beta},
$$

with the greek indices being generic, and with ${ }^{\alpha}[0]_{\beta}:=\delta_{\beta}^{\alpha}$ and ${ }_{\alpha}[0]^{\beta}:=\delta_{\alpha}^{\beta}$. The $a, b, d$ system of generating functions is a direct uplift of the $n, l$ system, as should be clear by setting $t=0$ or $u=0$. The usefulness of introducing these generalized generating functions is seen directly, when trying to relate $n$ with $l$ in a similar way, as $y$ was related to $s$ from (9.11) in the previous section: the equivalent expression of (9.11) here reads

$\operatorname{Tr}\left(\boldsymbol{N}^{n}\right)=\operatorname{Tr}\left(\boldsymbol{L}^{n}\right)+\sum_{i=0}^{n-1} \operatorname{Tr}\left(\boldsymbol{L}^{i}\right) \operatorname{Tr}\left(\boldsymbol{N}^{n-1-i}\right)-\sum_{i=0}^{n-2}(-1)^{n-2-i I}[2 i+1]_{A I}[2(n-2-i)+1]^{A}$,

valid for $n \geq 1$, and with the last term understood as zero for $n=1$. It can be equivalently written as

$$
n(t)-N=l(t)-2 M+t l(t) n(t)-t^{2} a(t,-t) .
$$

As described in appendix D.2, it is possible to relate the generators $a, b, d$ with each other, through similar, but much more involved manner, and as an outcome it is possible relate $n$ entirely in terms of $l$ (or vice-versa). The result is that

$$
n(t)=\frac{l(t)-2 M+N}{1-t l(t)\left(1-\frac{t}{(2 M-N+2) t-2}\right)} \quad \Leftrightarrow \quad l(t)=\frac{n(t)+2 M-N}{1+t n(t)\left(1-\frac{t}{(2 M-N+2) t-2}\right)},
$$

and as a bonus, we are also able to find expressions for $a, b, d$ in terms of $n$ and $l$, reading

$$
\begin{aligned}
a(t, u) & =\frac{l(t)-2 M-n(-u)+N}{(2 M-N+1) t u+t-u} \\
b(t, u) & =\frac{u n(-u)(t u-t-u)+t n(t)(t u+t+u-u n(-u)(t+u))}{(t+u)((2 M-N+1) t u+t-u)} \\
d(t, u) & =\frac{u l(-u)(t u-t-u)+t l(t)(t u+t+u+u l(-u)(t+u))}{(t+u)((2 M-N+1) t u+t-u)}
\end{aligned}
$$


Notice that $a(t, u)$ is well-defined at $u=-t$, and consistent with (9.33), while $b$ and $d$ seem singular at that point but the limit $u \rightarrow-t$ exists, as it should by definition.

Now that we have established a relation between $n$ and $l$ it is straightforward to pass this on to a relation between $m$ and $k$ : by formally rewriting $\sum_{n=0}^{\infty} t^{n} \operatorname{Tr}\left(\boldsymbol{O}^{n}\right)=\operatorname{Tr}\left(\frac{1}{1-t \boldsymbol{O}}\right)$ (cf. (9.9)) and using the relations in (9.27), the following pairwise relations between the generating functions are found:

$$
\begin{array}{rlrl}
m(t)=\frac{n\left(-\frac{t}{1-M t}\right)}{1-M t} & \Leftrightarrow & n(t)=\frac{m\left(-\frac{t}{1-M t}\right)}{1-M t}, \\
k(t)=\frac{l\left(-\frac{t}{1+\frac{N}{2} t}\right)}{1+\frac{N}{2} t} \quad \Leftrightarrow & l(t)=\frac{k\left(-\frac{t}{1+\frac{N}{2} t}\right)}{1+\frac{N}{2} t} .
\end{array}
$$

From these and (9.34) we finally establish

$$
\begin{gathered}
k(t)=\frac{(2 M-N)\left(1+\frac{2 M t}{2+N t}\right)+m\left(\frac{2 t}{2+(2 M+N) t}\right)}{\left(1+\frac{N}{2} t\right)\left(1+\frac{2 M t}{2+N t}\right)-\frac{1}{2} t\left(1+\frac{1+M t}{1+(1+M) t}\right) m\left(\frac{2 t}{2+(2 M+N) t}\right)}, \\
m(t)=-\frac{(2 M-N)\left(1-\frac{N t}{2-2 M t}\right)-k\left(\frac{2 t}{2-(2 M+N) t}\right)}{(1-M t)\left(1-\frac{N t}{2-2 M t}\right)+\frac{1}{2} t\left(1+\frac{2-N t}{2+(2-N) t}\right) k\left(\frac{2 t}{2-(2 M+N) t}\right)} .
\end{gathered}
$$

We notice that the expressions for $k$ and $m$ are symmetric under the exchange $N \leftrightarrow-2 M$ and $m(t) \leftrightarrow-k(t)$, while those for $n$ and $l$ are simply symmetric under $n(t) \leftrightarrow-l(t)$. This fits into the picture of universality (see, e.g. [108-110]) and the $S O_{N} \underset{N \leftrightarrow-2 M}{\longleftrightarrow} S p_{2 M}$ relation $[111,112]$.

It is worth checking the relations at the lowest orders without using the generating function method. The first two within each group read

$$
\begin{array}{rlrl}
\operatorname{Tr}(\boldsymbol{N}) & =M N, & \operatorname{Tr}(\boldsymbol{L}) & =-M N, \\
\operatorname{Tr}\left(\boldsymbol{N}^{2}\right) & =\mathcal{C}_{2}[\boldsymbol{M}]+M^{2} N, & \operatorname{Tr}\left(\boldsymbol{L}^{2}\right)=\mathcal{C}_{2}[\boldsymbol{K}]+\frac{N^{2} M}{2},
\end{array}
$$

where $\operatorname{Tr}(\boldsymbol{M})=\operatorname{Tr}(\boldsymbol{K})=0$ was used. Next, using

$$
\operatorname{Tr}\left(\boldsymbol{M}^{3}\right)=\left(\frac{N}{2}-1\right) \mathcal{C}_{2}[\boldsymbol{M}], \quad \operatorname{Tr}\left(\boldsymbol{K}^{3}\right)=-(1+M) \mathcal{C}_{2}[\boldsymbol{K}]
$$

one finds at the third and fourth orders

$$
\begin{aligned}
& \operatorname{Tr}\left(\boldsymbol{N}^{3}\right)=\left(1-\frac{N}{2}+3 M\right) \mathcal{C}_{2}[\boldsymbol{M}]+M^{3} N \\
& \operatorname{Tr}\left(\boldsymbol{L}^{3}\right)=\left(1+M-\frac{3 N}{2}\right) \mathcal{C}_{2}[\boldsymbol{K}]-\frac{M N^{3}}{4},
\end{aligned}
$$


and

$$
\begin{aligned}
\operatorname{Tr}\left(\boldsymbol{N}^{4}\right) & =\mathcal{C}_{4}[\boldsymbol{M}]+\left(6 M^{2}+4 M-2 M N\right) \mathcal{C}_{2}[\boldsymbol{M}]+M^{4} N \\
\operatorname{Tr}\left(\boldsymbol{L}^{4}\right) & =\mathcal{C}_{4}[\boldsymbol{K}]+\left(\frac{3}{2} N^{2}-2 N-2 M N\right) \mathcal{C}_{2}[\boldsymbol{K}]+\frac{M N^{4}}{8} .
\end{aligned}
$$

With these expressions one can now check the duality relations. For instance by moving the $y$ operator in the left end of $\operatorname{Tr}\left(\boldsymbol{N}^{n}\right)$ to its right end at $n=2$, we find

$$
\operatorname{Tr}\left(\boldsymbol{N}^{2}\right)=\operatorname{Tr}\left(\boldsymbol{L}^{2}\right)+\operatorname{Tr}\left(\boldsymbol{L}^{0}\right) \operatorname{Tr}(\boldsymbol{N})+\operatorname{Tr}(\boldsymbol{L}) \operatorname{Tr}\left(\boldsymbol{N}^{0}\right)-\operatorname{Tr}(\boldsymbol{L}),
$$

while at $n=4$ we find

$$
\begin{aligned}
\operatorname{Tr}\left(\boldsymbol{N}^{4}\right)= & \operatorname{Tr}\left(\boldsymbol{L}^{4}\right)+\operatorname{Tr}\left(\boldsymbol{L}^{0}\right) \operatorname{Tr}\left(\boldsymbol{N}^{3}\right)+\operatorname{Tr}(\boldsymbol{L}) \operatorname{Tr}\left(\boldsymbol{N}^{2}\right)+\operatorname{Tr}\left(\boldsymbol{L}^{2}\right) \operatorname{Tr}(\boldsymbol{N})+\operatorname{Tr}\left(\boldsymbol{L}^{3}\right) \operatorname{Tr}\left(\boldsymbol{N}^{0}\right) \\
& -\operatorname{Tr}\left(\boldsymbol{L}^{3}\right)+\operatorname{Tr}\left(\boldsymbol{L}^{0}\right) \operatorname{Tr}\left(\boldsymbol{N}^{2}\right)-\operatorname{Tr}\left(\boldsymbol{N}^{0}\right) \operatorname{Tr}\left(\boldsymbol{N}^{2}\right)+\operatorname{Tr}\left(\boldsymbol{N}^{2}\right) .
\end{aligned}
$$

From the above low-order relations it can now be checked that

$$
\begin{aligned}
\mathcal{C}_{2}[\boldsymbol{M}]= & \mathcal{C}_{2}[\boldsymbol{K}]+\frac{M N(2 M-N+2)}{2}, \\
\mathcal{C}_{4}[\boldsymbol{M}]= & \mathcal{C}_{4}[\boldsymbol{K}]-\frac{2 M+N}{2} \mathcal{C}_{2}[\boldsymbol{K}] \\
& +\frac{M N}{8}(2 M-N+2)((2 M-N)(2 M-N+2)+2 M N-2 N+4),
\end{aligned}
$$

which is exactly what one finds directly from (9.40).

Using the results of [107], one can derive a relation between generating functions of Casimir operators different from (9.40). This is due to relations between odd and even Casimirs of orthogonal and symplectic groups. We will comment more on different relations between generating functions in the follow-up paper.

\section{Acknowledgments}

The authors are grateful to Zhirayr Avetisyan and Axel Kleinschmidt for useful remarks and Minoru Itoh for communication on his work [107]. The work of T.B. and E.J. was supported by National Research Foundation (Korea) through the grants NRF2018H1D3A1A02074698 and NRF-2019R1F1A1044065. The work of K.M. was supported in part by Scuola Normale, by INFN (IS GSS-Pi) and by the MIUR-PRIN contract 2017CC72MK_003. K.M. also thanks Humboldt Foundation and Max-Planck Institute for Gravitational Physics (Albert-Einstein-Institute) for the support in the initial stages of this work.

\section{A Representations of compact groups}

Finite-dimensional representations of compact Lie groups are known to be in one-to-one correspondence with integral dominant weights. The latter are weights whose components 
in the Dynkin basis are all positive integers. In other words, finite-dimensional representations of compact Lie groups $G$ are highest weight representations, with highest weight of the form $\boldsymbol{\alpha}=\sum_{k=1}^{\operatorname{rank}(G)} \alpha_{k}^{\omega} \boldsymbol{\omega}_{k}$ with $\alpha_{k}^{\omega} \in \mathbb{N}$ the Dynkin labels and $\boldsymbol{\omega}_{k}$ the fundamental weights of $G$. In this appendix, we will review how these highest weight representations are realized as spaces of tensors with special properties. To do so, it will be more convenient to express the weights of $G$ in the orthonormal basis $\left\{\mathrm{e}_{k}\right\}_{k=1}^{\operatorname{rank}(G)}$, where the vectors $\mathrm{e}_{i}$ are mutually orthogonal unit vectors in $\mathbb{R}^{\operatorname{rank}(G)}$. We will write $\boldsymbol{\alpha}=\left(\alpha_{1}, \ldots, \alpha_{N}\right)$, for $\alpha_{1}, \ldots, \alpha_{N}$ the components of $\boldsymbol{\alpha}$ in this orthonormal basis.

\section{A.1 Finite-dimensional representations of $G L_{N}$}

Let us start with the reductive group $G L_{N}$. In the orthonormal basis, the components of a $G L_{N}$ integral dominant weight $\boldsymbol{\alpha}$ are all integers (not necessarily positive) and ordered, i.e.

$$
\alpha_{1} \geq \alpha_{2} \geq \cdots \geq \alpha_{N}
$$

Recall that the $G L_{N}$-irreps appearing in the decomposition of $V_{L}=\left(\mathbb{C}^{N}\right)^{\otimes L}$ are uniquely associated with the irreps of the symmetric group $\mathcal{S}_{L}$, defined by Young diagrams $\boldsymbol{\ell}$,

$$
\left.V_{L}\right|_{\mathcal{S}_{L} \times G L_{N}}=\bigoplus_{\ell \in \operatorname{Par}(L \mid N)} \ell \otimes[\ell]_{G L_{N}}
$$

where $\operatorname{Par}(L \mid N)$ denotes the set of Young diagrams with $L$ boxes and at most $N$ rows. This standard result is known as the Schur-Weyl duality, and can be seen as another instance of duality in representation theory, similar to the dual pair correspondence (see e.g. $[70,71,113]$ and references therein). The subspace of $V_{L}$ carrying the $\mathcal{S}_{L}$-irrep $\ell$ can be singled out by the projector $\mathbb{P}^{\ell}: V_{L} \rightarrow \ell$, i.e. the operator that symmetrizes a given tensor $T^{a_{1} \ldots a_{L}}$ so that the resulting tensor has the symmetry of the Young diagram $\boldsymbol{\ell}$. In other words, the $G L_{N}$ representation denoted $[\ell]_{G L_{N}}$ and dual to the $\mathcal{S}_{L}$-irrep $\boldsymbol{\ell}$ consists of a tensor with the symmetry of the Young diagram $\ell$.

For instance, when $L=2$ there are two possible representation of $\mathcal{S}_{2}$ to consider, namely $\boldsymbol{\ell}=(2)$ and $\boldsymbol{\ell}=(1,1)$, corresponding to a symmetric and an antisymmetric tensor. The associated projectors are

$$
\mathbb{P}_{a_{1} a_{2}}^{\frac{b_{1} b_{2}}{2}}=\frac{1}{2}\left(\delta_{a_{1}}^{b_{1}} \delta_{a_{2}}^{b_{2}}+\delta_{a_{1}}^{b_{2}} \delta_{a_{2}}^{b_{1}}\right), \quad \mathbb{P}_{a_{1} a_{2}}^{\frac{b_{2}}{b_{2}}}=\frac{1}{2}\left(\delta_{a_{1}}^{b_{1}} \delta_{a_{2}}^{b_{2}}-\delta_{a_{1}}^{b_{2}} \delta_{a_{2}}^{b_{1}}\right)
$$

For $L=3$, there are three possible Young diagrams that can appear (for $N>2$ ), namely $\boldsymbol{\ell}=(3), \boldsymbol{\ell}=(1,1,1)$ and $\boldsymbol{\ell}=(2,1)$. Only one standard Young tableau can be attached to 
the first two, while two for the last diagram. The associated projectors read

$$
\begin{aligned}
& \mathbb{P}_{a_{1} a_{2} a_{3}}^{\frac{b_{1} b_{2} b_{3}}{3 !}}=\frac{1}{3 !}\left(\delta_{a_{1}}^{b_{1}} \delta_{a_{2}}^{b_{2}} \delta_{a_{3}}^{b_{3}}+\delta_{a_{1}}^{b_{1}} \delta_{a_{2}}^{b_{3}} \delta_{a_{3}}^{b_{2}}+\delta_{a_{1}}^{b_{2}} \delta_{a_{2}}^{b_{1}} \delta_{a_{3}}^{b_{3}}+\delta_{a_{1}}^{b_{2}} \delta_{a_{2}}^{b_{3}} \delta_{a_{3}}^{b_{1}}+\delta_{a_{1}}^{b_{3}} \delta_{a_{2}}^{b_{2}} \delta_{a_{3}}^{b_{1}}+\delta_{a_{1}}^{b_{3}} \delta_{a_{2}}^{b_{1}} \delta_{a_{3}}^{b_{2}}\right), \\
& \mathbb{P}_{a_{1} a_{2} a_{3}}^{\frac{b_{3}}{b_{3}}}=\frac{1}{3 !}\left(\delta_{a_{1}}^{b_{1}} \delta_{a_{2}}^{b_{2}} \delta_{a_{3}}^{b_{3}}-\delta_{a_{1}}^{b_{1}} \delta_{a_{2}}^{b_{3}} \delta_{a_{3}}^{b_{2}}-\delta_{a_{1}}^{b_{2}} \delta_{a_{2}}^{b_{1}} \delta_{a_{3}}^{b_{3}}+\delta_{a_{1}}^{b_{2}} \delta_{a_{2}}^{b_{3}} \delta_{a_{3}}^{b_{1}}-\delta_{a_{1}}^{b_{3}} \delta_{a_{2}}^{b_{2}} \delta_{a_{3}}^{b_{1}}+\delta_{a_{1}}^{b_{3}} \delta_{a_{2}}^{b_{1}} \delta_{a_{3}}^{b_{2}}\right) \\
& \mathbb{P}_{a_{1} a_{2} a_{3}}^{\frac{b_{3} b_{2}}{b_{3}}}=\frac{1}{3}\left(\delta_{a_{1}}^{b_{1}} \delta_{a_{2}}^{b_{2}} \delta_{a_{3}}^{b_{3}}+\delta_{a_{1}}^{b_{2}} \delta_{a_{2}}^{b_{1}} \delta_{a_{3}}^{b_{3}}-\delta_{a_{1}}^{b_{2}} \delta_{a_{2}}^{b_{3}} \delta_{a_{3}}^{b_{1}}-\delta_{a_{1}}^{b_{3}} \delta_{a_{2}}^{b_{2}} \delta_{a_{3}}^{b_{1}}\right),
\end{aligned}
$$

and

$$
\mathbb{P}_{a_{1} a_{2} a_{3}}=\frac{1}{3}\left(\delta_{a_{1}}^{b_{1}} \delta_{a_{2}}^{b_{2}} \delta_{a_{3}}^{b_{3}}-\delta_{a_{1}}^{b_{2}} \delta_{a_{2}}^{b_{1}} \delta_{a_{3}}^{b_{3}}-\delta_{a_{1}}^{b_{3}} \delta_{a_{2}}^{b_{1}} \delta_{a_{3}}^{b_{2}}+\delta_{a_{1}}^{b_{3}} \delta_{a_{2}}^{b_{2}} \delta_{a_{3}}^{b_{1}}\right) .
$$

The action of $G L_{N}$ on $\left[\ell=\left(\ell_{1}, \ldots, \ell_{p}\right)\right]_{G L_{N}}$ with $p \leq N$ reads

$$
\rho_{\ell}(g) T^{a_{1}\left(\ell_{1}\right), \ldots, a_{p}\left(\ell_{p}\right)}=\prod_{k=1}^{p} \ell_{k} g_{b}^{a_{k}} T^{a_{1}\left(\ell_{1}\right), \ldots, b a_{k}\left(\ell_{k}-1\right), \ldots, a_{p}\left(\ell_{p}\right)},
$$

where $g_{b}{ }^{c}$ denote the components of the matrix $g \in G L_{N}$. Correspondingly, the action of the Lie algebra $\mathfrak{g l}_{N}$ is given by

$$
X_{c}^{b} T^{a_{1}\left(\ell_{1}\right), \ldots, a_{p}\left(\ell_{p}\right)}=\sum_{k=1}^{N} \ell_{k} \delta_{c}^{a_{k}} T^{a_{1}\left(\ell_{1}\right), \ldots, b a_{k}\left(\ell_{k}-1\right), \ldots, a_{p}\left(\ell_{p}\right)} .
$$

Recall that these generators obey

$$
\left[X_{b}^{a}, X_{d}^{c}\right]=\delta_{b}^{c} X_{d}^{a}-\delta_{d}^{a} X_{b}^{c},
$$

from which we can see that $X_{a}^{a}$ generate a Cartan subalgebra, while $X^{a}{ }_{b}$ for $a<b$ (resp. $a>b)$ are raising (resp. lowering) operators. Let us show that the component $T^{1\left(\ell_{1}\right), \ldots, p\left(\ell_{p}\right)}$ of this tensor (i.e. the component obtained by setting all the indices of $a$-th group of symmetric indices to the value $a$ ) is the highest weight vector of this $\mathfrak{g l}_{N}$ irrep. For this particular component, the above action simplifies to a single term,

$$
X_{b}^{a} T^{1\left(\ell_{1}\right), \ldots, p\left(\ell_{p}\right)}=\ell_{b} T^{1\left(\ell_{1}\right), \ldots, a b\left(\ell_{b}-1\right), \ldots, p\left(\ell_{p}\right)},
$$

when $b \leq p$, and vanishes otherwise. First of all, it is an eigenvector of the Cartan subalgebra generator $X_{a}^{a}$ (no summation implied), with eigenvalue $\ell_{a}$. The raising operator are the generators $X_{b}^{a}$ with $a<b$, which implies that their action takes the form

$$
X_{b}^{a} T^{1\left(\ell_{1}\right), \ldots, p\left(\ell_{p}\right)}=\ell_{b} T^{1\left(\ell_{1}\right), \ldots, a\left(\ell_{a}\right), \ldots, a b\left(\ell_{b}-1\right), \ldots, p\left(\ell_{p}\right)}=0,
$$

i.e. all the indices of the $a$-th group are symmetrized with one index of a latter group (the $b$-th), which identically vanishes due to the symmetry of the tensor $T .{ }^{19}$ This shows that

\footnotetext{
${ }^{19}$ For instance, in the case of $\boldsymbol{\ell}=(2,1)$, the action of a generator $X^{i}{ }_{j}$ of $\mathfrak{g l}_{N}$ reads

$$
X_{d}^{c} T^{a_{1} a_{2}, b}=2 \delta_{d}^{\left(a_{1}\right.} T^{\left.a_{2}\right) c, b}+\delta_{d}^{b} T^{a_{1} a_{2}, c} .
$$
}


the representation of $G L_{N}$ on the space of tensor with the symmetry of the Young diagram $\boldsymbol{\ell}$ is a highest weight representation, with highest weight given by $\boldsymbol{\ell}$ (in the orthonormal basis).

However, this does not exhaust all possible finite-dimensional representations. So, what about representations with highest weights containing some negative components? It turns out that they can be realized as a subspace of

$$
V_{L, M}=\left(\mathbb{C}^{N}\right)^{\otimes L} \otimes\left(\mathbb{C}^{N *}\right)^{\otimes M},
$$

i.e. they correspond to spaces of tensors $T$ with two types of indices: $T_{b_{1} \cdots b_{M}}^{a_{1} \cdots a_{L}}$.

First, let us have a look at $V_{0,1}=\mathbb{C}^{N *}$. As a vector space, this corresponds to the dual space of the fundamental representation. As such, it also carries a representation $\rho$ of $G L_{N}$ defined by

$$
\left\langle\rho_{\bar{f}}(g) \phi, v\right\rangle=\left\langle\phi, \rho_{f}(g) v\right\rangle,
$$

for $g \in U(N), v \in \mathbb{C}^{N}$ and $\phi \in \mathbb{C}^{N *}$, and where $\langle$,$\rangle denote the pairing between \mathbb{C}^{N}$ and $\mathbb{C}^{N *}$. More concretely, this means that

$$
\rho_{\bar{f}}(g)=\rho_{f}\left(g^{-1}\right)^{t}
$$

where the exponent ${ }^{t}$ denote the transpose. The action of $G L_{N}$ on $V_{0, M}$ is then simply the tensor product of $\rho_{\bar{f}}$. The same arguments as previously show that $V_{0, M}$ can be decomposed into a direct sum of $\mathcal{S}_{L} \otimes G L_{N}$ irreps, which are in bijections. In other words, irreducible representations of $G L_{N}$ in $V_{0, M}$ correspond to tensors with the symmetry of all possible Young diagrams $\boldsymbol{m}$ with $M$ boxes and at most $N$ rows. The corresponding action of $\mathfrak{g l}_{N}$ on such tensors is given by

$$
X_{c}^{b} T_{a_{1}\left(m_{1}\right), \ldots, a_{p}\left(m_{p}\right)}=-\sum_{k=1}^{N} m_{k} \delta_{a_{k}}^{b} T_{a_{1}\left(m_{1}\right), \ldots, b a_{k}\left(m_{k}-1\right), \ldots, a_{p}\left(m_{p}\right)} .
$$

In this case, the component $T_{N\left(m_{1}\right), \ldots,(N-p)\left(m_{p}\right)}$ is the highest weight of the representation. The action of $\mathfrak{g l}_{N}$ now reads

$$
X^{b}{ }_{c} T_{N\left(m_{1}\right), \ldots, 1\left(m_{p}\right)}=-m_{N+1-b} T_{N\left(m_{1}\right), \ldots, c(N+1-b)\left(m_{b}-1\right), \ldots,(N+1-p)\left(m_{p}\right)},
$$

if $b \leq p$ and is trivial otherwise. It is clear that $T_{N\left(m_{1}\right), \ldots,(N+1-p)\left(m_{p}\right)}$ is an eigenvector of all generators of the Cartan subalgebra, with weight $\left(0, \ldots, 0,-m_{p}, \ldots,-m_{1}\right)$. Moreover, for $b<c$ the previous equation reads

$$
X_{c}^{b} T_{N\left(m_{1}\right), \ldots, 1\left(m_{p}\right)}=-m_{N+1-b} T_{N\left(m_{1}\right), \ldots, c\left(m_{N+1-c}\right), \ldots, c(N+1-b)\left(m_{b}-1\right), \ldots,(N+1-p)\left(m_{p}\right)}=0,
$$

due to the symmetry of $T$. This shows that tensor on $V_{0, L}$ having the symmetry of a Young diagram $\boldsymbol{m}$ form a highest weight representation of $G L_{N}$ with highest weight $\left(0, \ldots, 0,-m_{p}, \ldots,-m_{1}\right)$, i.e. whose components are all negative.

For $a_{1}=a_{2}=1$ and $b=2$ and all pairs $c>d$, the above vanishes: the first term because of the factor $\delta_{d}^{1}$ with $d>1$ and the second term because of the fact that the total symmetrization of all indices of $T$ vanishes and hence $T^{11,1}=0$. 
Finally, consider the case $L=M=1$, i.e. the space of tensors of the form $T_{b}^{a}$, on which $G L_{N}$ acts as

$$
\rho_{V_{1,1}}(g) T_{b}^{a}=g_{c}{ }^{a}\left(g^{-1}\right)_{b}{ }^{d} T_{d}^{c},
$$

for $g \in G L_{N}$. In particular, one can notice that the trace of $T=\delta_{a}^{b} T_{b}^{a}$ defines a onedimensional invariant subspace of $V_{1,1}$, as $\rho_{V_{1,1}}(g) T=T$. This observation extends to higher rank tensors, and hence we can conclude that the traces of any tensor (in the sense of any contraction of an upper index with a lower one) define a invariant subspace. In other words, the irreducible representations of $G L_{N}$ in $V_{L, M}$ are composed of traceless tensors. Added to the previous discussions, we can conclude that highest weight representations of $G L_{N}$, with highest weight

$$
\left(\ell_{1}, \ldots, \ell_{p}, 0, \ldots, 0,-m_{q}, \ldots,-m_{1}\right)
$$

where $p+q \leq N$ can be realized as the space of tensors of the form

$$
T_{b_{1}\left(m_{1}\right), \ldots, b_{q}\left(m_{q}\right)}^{a_{1}\left(\ell_{1}\right), \ldots, a_{p}\left(\ell_{p}\right)}
$$

i.e. whose upper indices have the symmetry of the Young diagram $\boldsymbol{\ell}=\left(\ell_{1}, \ldots, \ell_{p}\right)$, lower indices the symmetry of the Young diagram $\boldsymbol{m}=\left(m_{1}, \ldots, m_{q}\right)$, and which are traceless. Notice that the tracelessness implies that the tensors with $p+q \geq N$ identically vanish.

It will be useful to record the expression of the dimension of the space of tensors with symmetry $\ell$,

$$
\operatorname{dim}[\ell]_{G L_{N}}=\prod_{1 \leq i<j \leq N} \frac{\ell_{i}-\ell_{j}+j-i}{j-i} .
$$

The finite dimensional representations of $G L_{N}$ described above provide all unitary irreps of $U(N)$, which are isomorphic to the unitary irreps of $U(1) \times S U(N)$. The $U(1)$ irrep is simply given by $|\boldsymbol{\ell}|-|\boldsymbol{m}|$, the number of boxes in $\boldsymbol{\ell}$ minus the number of boxes in $\boldsymbol{m}$. An $S U(N)$ irrep is again given by a Young diagram now with maximum height $N-1$. In the following, we demonstrate how a single Young diagram of $S U(N)$ irrep can be obtained from the two Young diagrams $\boldsymbol{\ell}$ and $\boldsymbol{m}$ of a $U(N)$ irrep. Without the traceless condition, each of $\boldsymbol{\ell}$ and $\boldsymbol{m}$ carry an irrep of $S U(N)$, and hence we get a tensor product representation which we need to decompose. To do so, we express the diagram $\boldsymbol{m}$ in the antisymmetric basis, as $\boldsymbol{m}=\left[m^{1}, \ldots, m^{r}\right]$ where $m^{k}$ are the height of the $k$-th column (hence $m^{1}=q$ and $r=m_{q}$ ). The corresponding tensor $\Psi$ now has the form

$$
\Psi_{j^{1}\left[m^{1}\right], \ldots, j^{r}\left[m^{r}\right]}^{i_{1}\left(\ell_{1}, \ldots, i_{p}\left(\ell_{p}\right)\right.}
$$

i.e. $j^{k}\left[m^{k}\right]$ denote a group of $m^{k}$ indices which are antisymmetrized. Then, we define a dual tensor $\tilde{\Psi}$ by applying contracting each group of antisymmetric indices with $\epsilon^{a[N]}$, the totally antisymmetric (Levi-Civita) symbol:

$$
\tilde{\Psi}^{i_{1}\left(\ell_{1}\right), \ldots, i_{p}\left(\ell_{p}\right) ; j^{1}\left[N-m^{1}\right], \ldots, j^{r}\left[N-m^{r}\right]}=\epsilon^{j^{1}[N]} \cdots \epsilon^{j^{r}[N]} \Psi_{j^{1}\left[m^{1}\right], \ldots, j^{r}\left[m^{r}\right]}^{i_{1}\left(\ell_{1}\right), \ldots, i_{p}\left(\ell_{p}\right)} .
$$

The dual tensor $\tilde{\Psi}$ has now two types of upper indices: the first $p$ groups have the symmetry $\ell$, while the next $r$ groups (resulting from dualization) have the symmetry of the dualized 


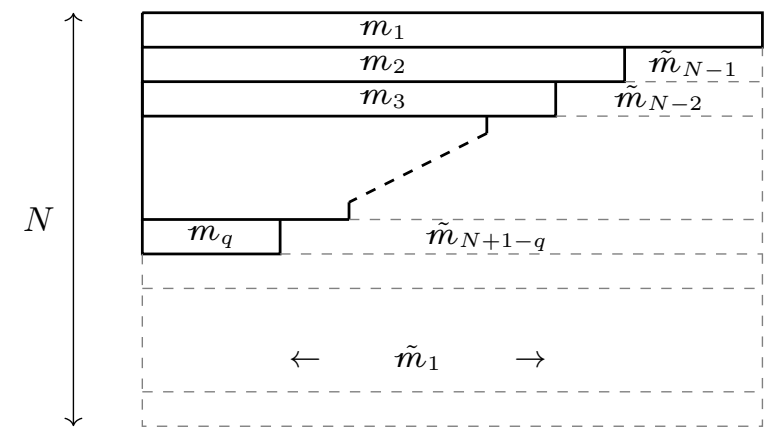

Figure 1. A Young diagram $\boldsymbol{m}$ (black) and its dual $\tilde{\boldsymbol{m}}$ (gray).

Young diagram $\tilde{\boldsymbol{m}}=\left[\tilde{m}^{1}, \ldots, \tilde{m}^{r}\right]$ with $\tilde{m}^{k}=N-m^{k}$. In other words, the dual tensor $\tilde{\Psi}$ has the symmetry of the tensor product $\boldsymbol{\ell} \otimes \tilde{\boldsymbol{m}}$, which we should try to decompose. In the symmetric basis, $\tilde{\boldsymbol{m}}=\left(\tilde{m}_{1}, \tilde{m}_{2}, \ldots\right)$ is given by (see figure 1 ):

$$
\tilde{m}_{k}=m_{1}-m_{N+1-k} .
$$

Now recall that $\Psi$ is traceless. On the dual tensor $\tilde{\Psi}$, the traceless condition for $\Psi$ translates to

$$
0=\tilde{\Psi}^{\cdots\left[i_{k} \cdots ; \cdots \mid j^{l}\left[N-m^{l}\right]\right] \cdots},
$$

This implies that, when applying Littlewood-Richardson rule to decompose $\boldsymbol{\ell} \otimes \tilde{\boldsymbol{m}}$, no antisymmetrization of an index in $\boldsymbol{\ell}$ with a column of $\tilde{\boldsymbol{m}}$ is allowed. Such a diagram occurs only when $p \leq N-q$, that is, $p+q \leq N$ and is unique. This diagram is obtained by simply attaching $\boldsymbol{\ell}$ to the right side of $\tilde{\boldsymbol{m}}$ :

$$
\begin{aligned}
& \left(\tilde{m}_{1}+\ell_{1}, \tilde{m}_{1}+\ell_{2}, \ldots\right) \\
& =(\underbrace{m_{1}+\ell_{1}, m_{1}+\ell_{2}, \ldots, m_{1}+\ell_{p}}_{p}, \underbrace{m_{1}, \ldots, m_{1}}_{N-p-q}, \underbrace{m_{1}-m_{q}, m_{1}-m_{q-1}, \ldots, 0}_{q}) .
\end{aligned}
$$

To recapitulate, due to the isomorphism $U(N) \cong U(1) \ltimes S U(N)$, the representations of $U(N)$ correspond to the tensor product of a $U(1)$ and a $S U(N)$ irrep. More precisely,

$$
[\boldsymbol{\ell} \oslash \boldsymbol{m}]_{U(N)}=[|\boldsymbol{\ell}|-|\boldsymbol{m}|]_{U(1)} \otimes[\tilde{\boldsymbol{m}}+\boldsymbol{\ell}]_{S U(N)},
$$

where by $\tilde{\boldsymbol{m}}+\boldsymbol{\ell}$, we mean the Young diagram (A.28) obtained by attaching $\tilde{\boldsymbol{m}}$ and $\boldsymbol{\ell}$.

\section{A.2 Finite-dimensional representations of $O(N)$}

Finite-dimensional representations of $O(N)$ can also be obtained by restriction from the tensor representations of $G L_{N}$ constructed previously. The orthogonal group $O(N)$ possesses an invariant tensor, namely the metric $\delta_{i j}$. As one can raise and lower indices with it, it is sufficient to consider tensors with only one kind of indices. The restriction of $[\boldsymbol{\ell}]_{G L_{N}}$ to $O(N)$ is however not irreducible: it contains invariant subspaces generated by traces taken with respect to $\delta_{i j}$. For instance, $[(2)]_{G L_{N}}$ which is the space of symmetric tensors 
$T_{i j}$ contains two irreducible representations of $O(N)$, corresponding to its trace $T=\delta^{i j} T_{i j}$ and its traceless part $\hat{T}_{i j}=T_{i j}-\frac{1}{N} \delta_{i j} T$.

Requiring a tensor to be traceless imposes some restriction on the possible symmetry $\boldsymbol{\ell}$ it can have. To see that, let us have a look at a rank $k+l$ tensor with the symmetry of the Young diagram $[k, l]$ in the antisymmetric basis (i.e. a two-column Young diagram, of respective height $k$ and $l$ ). It has

$$
\frac{k-l+1}{k+1}\left(\begin{array}{c}
N \\
k
\end{array}\right)\left(\begin{array}{c}
N+1 \\
l
\end{array}\right)
$$

independent components, and its trace being a tensor with symmetry $[k-1, l-1]$. As a consequence, the traceless part of such a tensor has

$$
\frac{N !(N+1) !(k-l+1)}{(k+1) ! l !(N-k+1) !(N-l+2) !}[(N-k+1)(N-l+2)-(k+1) l],
$$

independent components. This number vanishes for $k+l=N+1$, and becomes negative when $k+l$ becomes larger. This means that a tensor $T$ with shape $[k, l]$ is necessarily "pure trace", i.e. of the form

$$
T_{\left[i_{1} \ldots i_{k}\right],\left[j_{1} \ldots j_{l}\right]}=\delta_{\left[i_{1} \mid\left[j_{1}\right.\right.} U_{\left.\left.i_{2} \ldots i_{k}\right], j_{2} \ldots j_{l}\right]},
$$

with some tensor $U$ of symmetry $[k-1, l-1]$. The same conclusion applies to more general tensor: if the corresponding Young diagram $\ell=\left[\ell^{1}, \ell^{2}, \ldots, \ell^{r}\right]$ is such that $\ell^{1}+\ell^{2}>N$, then its traceless part identically vanishes. As a consequence, finite-dimensional representations of $O(N)$ correspond to traceless tensor having the symmetry of a Young diagram $\boldsymbol{\ell}$ such that the sum of the height of its first two columns is at most $N$.

Highest weight of the representation. In order to show that the $O(N)$ irrep consisting of traceless tensor with symmetry $\ell$ is an highest weight module, it will be useful to work in the complexified basis where the metric takes the form

$$
J=\left(\begin{array}{cc}
0 & \mathbb{1}_{n} \\
\mathbb{1}_{n} & 0
\end{array}\right), \quad[\text { if } N=2 n] \quad \text { or } \quad J=\left(\begin{array}{ccc}
0 & \mathbb{1}_{n} & 0 \\
\mathbb{1}_{n} & 0 & 0 \\
0 & 0 & 1
\end{array}\right), \quad[\text { if } N=2 n+1],
$$

The generators $M_{A B}$ of $\mathfrak{s o}(N)$ obey

$$
\left[M_{A B}, M_{C D}\right]=J_{B C} M_{A D}-J_{A C} M_{B D}-J_{B D} M_{A C}+J_{A D} M_{B C},
$$

where $J_{A B}$ are the components of the matrix $J$ introduced above. The reality condition reads $M_{A B}{ }^{\dagger}=-J_{A C} J_{B D} M_{C D}$. When $N=2 n$, the index $A$ takes the values $A= \pm a$ with $a=1, \ldots, n$ and the only non-vanishing components of $J$ are $J_{+a-b}=\delta_{a b}$. When $N=2 n+1$, the index $A$ can assume one additional value which we will denote $*$, and $J_{* A}=\delta_{* A}$. Splitting the generators $M_{A B}$ accordingly, the previous commutation relations take the form

$$
\begin{aligned}
& {\left[M_{+a+b}, M_{-c-d}\right]=\delta_{a d} M_{+b-c}-\delta_{b d} M_{+a-c}-\delta_{a c} M_{+b-d}+\delta_{b c} M_{+a-d},} \\
& {\left[M_{+a-b}, M_{+c+d}\right]=2 \delta_{b[d} M_{+c]+a}, \quad\left[M_{+a-b}, M_{-c-d}\right]=-2 \delta_{a[d} M_{-c]-b},} \\
& {\left[M_{+a-b}, M_{+c-d}\right]=\delta_{b c} M_{+a-d}-\delta_{a d} M_{+c-b} .}
\end{aligned}
$$




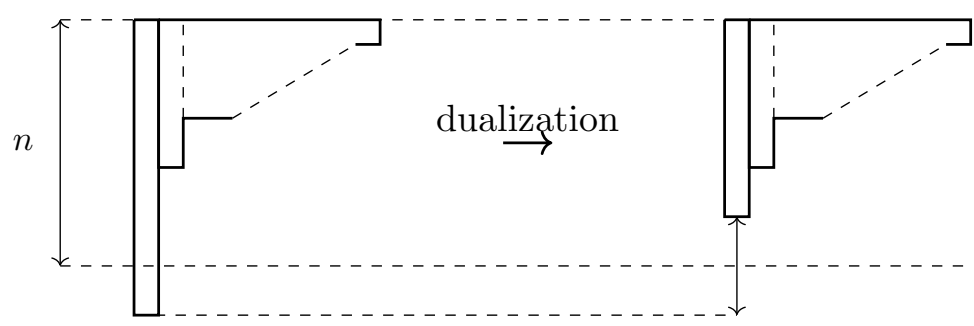

Figure 2. Example of a Young diagram with a first column higher than $n$ (left) and the Young diagram obtained after dualization (right).

The last commutator shows that the generators $M_{+a-b}$ span a $\mathfrak{u}(n)$ subalgebra. When $N=2 n+1$, there are $2 n$ additional generators, denoted $N_{ \pm a}=M_{ \pm a *}$, to take into account:

$$
\begin{aligned}
{\left[M_{+a-b}, N_{+c}\right] } & =\delta_{b c} N_{+a}, & {\left[M_{+a-b}, N_{-c}\right] } & =-\delta_{a c} N_{-b}, \\
{\left[M_{ \pm a \pm b}, N_{\mp c}\right] } & =\delta_{c[b} N_{ \pm a]}, & {\left[N_{\epsilon a}, N_{\varepsilon b}\right] } & =-M_{\epsilon a \varepsilon b},
\end{aligned}
$$

where $\epsilon, \varepsilon= \pm$ and all other commutators vanish. From these commutators, we can see that $M_{+a-a}$ generates a Cartan subalgebra ${ }^{20}$ while $M_{+a+a}, N_{+c}$ and $M_{+a-b}$ for $a<b$ (resp. $M_{-a-a}$ and $N_{-c}$ and $M_{+a-b}$ for $a>b$ ) are raising (resp. lowering) operators. The action of $\mathfrak{s o}(N)$ on a tensor of symmetry $\ell=\left(\ell_{1}, \ldots, \ell_{p}\right)$, with $p \leq N$, reads

$$
M_{A B} T_{C_{1}\left(\ell_{1}\right), \ldots, C_{p}\left(\ell_{p}\right)}=2 \sum_{k=1}^{p} \ell_{k} J_{C_{k}[B} T_{\left.C_{1}\left(\ell_{1}\right), \ldots, A\right] C_{k}\left(\ell_{k}-1\right), \ldots, C_{p}\left(\ell_{p}\right)} .
$$

We saw previously that if $T$ is traceless, then $\ell^{1}+\ell^{2} \leq N$. This implies that only the first column can be higher than $n$. When it is the case, i.e. when $\ell^{1} \geq n$, we can dualize this tensor (as we did in the previous subsection):

$$
\tilde{T}_{A_{1}\left[\tilde{\ell}^{1}\right], A_{2}\left[\ell^{2}\right], \ldots, A_{r}\left[\ell_{r}\right]}=\epsilon^{A_{1}[N]} T_{A_{1}\left[\ell^{1}\right], A_{2}\left[\ell^{2}\right], \ldots, A_{r}\left[\ell^{r}\right]},
$$

with $\tilde{\ell}^{1}=N-\ell^{1} \leq n$ and $\ell=\left[\ell^{1}, \ldots, \ell^{r}\right]$ in the antisymmetric basis (i.e. $\ell^{1}=p$ is the number of rows and $r=\ell_{1}$ the number of columns). The dual tensor $\tilde{T}$ now has the symmetry of the dual Young diagram

$$
\tilde{\ell}:=\left[N-\ell^{1}, \ell^{2}, \ldots, \ell^{r}\right]=(\ell_{1}, \ldots, \ell_{p}, \underbrace{1, \ldots, 1}_{N-2 p}),
$$

which, in particular than $n$ rows (see figure 2). With this in mind, we can now assume (without loss of generality) that $p \leq n$, i.e. consider tensor corresponding to Young diagrams with less than $n$ non-empty rows. Now let us show that the component $T_{+1\left(\ell_{1}\right), \ldots,+p\left(\ell_{p}\right)}$ is the highest weight vector of the representation. First of all, we can immediately see that

\footnotetext{
${ }^{20}$ Notice that the Killing form $\kappa$ of $\mathfrak{s o}(N)$ is given by $\kappa\left(M_{A B}, M_{C D}\right) \propto J_{A[C} J_{D] B}$ so that the Cartan subalgebra generators $H_{a}=M_{+a-a}$ verify $\kappa\left(H_{a}, H_{b}\right)=\delta_{a b}$ (upon choosing the proper normalization). As a consequence, their highest weight computed hereafter will be obtained in the orthonormal basis.
} 
it is annihilated by all $M_{+a+b}$ and $N_{+c}$. Secondly, the action of the generators $M_{+a-b}$ on this component simply takes the form

$$
M_{+a-b} T_{+1\left(\ell_{1}\right), \ldots,+p\left(\ell_{p}\right)}=\ell_{b} T_{+1\left(\ell_{1}\right), \ldots,+a+b\left(\ell_{b}-1\right), \ldots,+p\left(\ell_{p}\right)} .
$$

When $a=b$, i.e. for a Cartan subalgebra generator, this reduces to

$$
M_{+a-a} T_{+1\left(\ell_{1}\right), \ldots,+p\left(\ell_{p}\right)}=\ell_{a} T_{+1\left(\ell_{1}\right), \ldots,+a\left(\ell_{a}\right), \ldots,+p\left(\ell_{p}\right)},
$$

that is, this component is an eigenvector of $M_{+a-a}$ with eigenvalue $\ell_{a}$. When $a<b$, i.e. for a raising operator, this simplifies to

$$
M_{+a-b} T_{+1\left(\ell_{1}\right), \ldots,+p\left(\ell_{p}\right)}=\ell_{b} T_{+1\left(\ell_{1}\right), \ldots,+a\left(\ell_{a}\right), \ldots,+a+b\left(\ell_{b}-1\right), \ldots,+p\left(\ell_{p}\right)}=0,
$$

which vanishes as a consequence of the symmetry of the tensor $T$. To summarized, we have proved that the $O(N)$ irrep defined by traceless tensor with symmetry $\boldsymbol{\ell}$ is an $S O(N)$ highest weight module with highest weight $\ell$ if the diagram has at most $n$ rows, or highest weight $\tilde{\ell}$ (dualized diagram) otherwise.

\section{A.3 Finite-dimensional representations of $S p(N)$}

Finite-dimensional representations of $S p(N)$ can also be obtained by restriction from the tensor representations of $G L_{2 N}$ constructed previously. The symplectic group $\operatorname{Sp}(N)$ possesses an invariant tensor, namely the canonical symplectic matrix $\Omega_{i j}$. Being nondegenerate, one can raise and lower indices with it, so that it is sufficient to consider tensors with one kind of indices. The restriction of $[\ell]_{G L_{2 N}}$ to $S p(N)$ is not irreducible: it contains invariant subspaces generated by traces taken with respect to $\Omega$. For instance, $[(1,1)]_{G L_{2 N}}$ which is the space of antisymmetric tensors $T_{i, j}$ contains two irreducible representations of $S p(N)$, corresponding to its trace $T=\Omega^{i j} T_{i, j}$ and its traceless part $\hat{T}_{i, j}=T_{i, j}+\frac{1}{2 N} \Omega_{i j} T$.

Requiring a tensor to be traceless imposes some restriction on the possible symmetry $\boldsymbol{\ell}$ it can have. Consider for instance a totally antisymmetric tensor of rank $k$. It has $\left(\begin{array}{c}2 N \\ k\end{array}\right)$ independent components, and its trace being a rank $k-2$ antisymmetric tensor has $\left(\begin{array}{c}2 N \\ k-2\end{array}\right)$. As a consequence, the traceless part of a rank $k$ antisymmetric tensor has

$$
\left(\begin{array}{c}
2 N \\
k
\end{array}\right)-\left(\begin{array}{c}
2 N \\
k-2
\end{array}\right)=\frac{2 N !}{k !(2 N-k+2) !}[(2 N-k+2)(2 N-k+1)-k(k-1)],
$$

independent components. This number vanishes for $k=N+1$, and becomes negative when $k$ becomes larger. This means that an antisymmetric tensor $T$ of rank $k>N$ is necessarily "pure trace", i.e. of the form

$$
T_{\left[i_{1} \ldots i_{k}\right]}=\Omega_{\left[i_{1} i_{2}\right.} U_{\left.i_{3} \ldots i_{k}\right]},
$$

with some rank $k-2$ antisymmetric tensor $U$. This arguments extends to tensors with more general symmetry, leading to the conclusion that tensors finite-dimensional representations of $S p(N)$ correspond to traceless tensor having the symmetry of a Young diagram $\boldsymbol{\ell}$ with at most $N$ rows. 
Highest weight of the representation. Recall that the algebra $\mathfrak{s p}(N)$ is spanned by symmetric generators $K_{I J}=K_{J I}$ with the index $A$ taking $2 N$ values and subject to the commutation relations

$$
\left[K_{I J}, K_{K L}\right]=\Omega_{J K} K_{I L}+\Omega_{J L} K_{I K}+\Omega_{I K} K_{J L}+\Omega_{I L} K_{J K} .
$$

Splitting the index into $A= \pm a$ with $a=1, \ldots, N$, and such that the only non-vanishing components of the symplectic matrix are $\Omega_{-a+b}=\delta_{a b}$, the above relations now read

$$
\begin{aligned}
& {\left[K_{+i+j}, K_{-k-l}\right]=\delta_{j k} K_{+i-l}+\delta_{j l} K_{+i-k}-\delta_{i k} K_{+j-l}-\delta_{i l} K_{+j-k},} \\
& {\left[K_{+i-j}, K_{+k+l}\right]=2 \delta_{j(k} K_{+l)+i}, \quad\left[K_{+i-j}, K_{-k-l}\right]=-2 \delta_{i(k} K_{-l)-j},} \\
& {\left[K_{+i-j}, K_{+k-l}\right]=\delta_{j k} K_{+i-l}-\delta_{i l} K_{-j+k} .}
\end{aligned}
$$

From this commutators, we can see that $K_{+i-i}$ generate a Cartan subalgebra, ${ }^{21}$ while $K_{+j+k}$ (resp. $K_{+j+k}$ ) and $K_{+l-m}$ for $l<m$ (resp. for $l>m$ ) are raising (resp. lowering) operators. Notice also that the last commutation relation shows that $K_{+i-j}$ forms a $\mathfrak{u}(N)$ subalgebra in $\mathfrak{s p}(N)$. The action of $\mathfrak{s p}(N)$ on a tensor of symmetry $\boldsymbol{\ell}=\left(\ell_{1}, \ldots, \ell_{p}\right)$, with $p \leq N$, is given by

$$
K_{I J} T_{L_{1}\left(\ell_{1}\right), \ldots, L_{p}\left(\ell_{p}\right)}=-2 \sum_{k=1}^{p} \ell_{k} \Omega_{L_{k}(I} T_{\left.L_{1}\left(\ell_{1}\right), \ldots, J\right) L_{k}\left(\ell_{k}-1\right), \ldots, L_{p}\left(\ell_{p}\right)} .
$$

Similarly to the previous cases, let us show that the component $T_{+1\left(\ell_{1}\right), \ldots,+p\left(\ell_{p}\right)}$ defines a highest weight vector. First, notice that $K_{+i+j}$ on this component is trivial. Second, the action of $K_{+i-j}$ reduces to

$$
K_{+i-j} T_{+1\left(\ell_{1}\right), \ldots,+p\left(\ell_{p}\right)}=\ell_{j} T_{+1\left(\ell_{1}\right), \ldots,+i+j\left(\ell_{j}-1\right), \ldots,+p\left(\ell_{p}\right)} .
$$

If $i=j$, i.e. we are looking at the action of the Cartan subalgebra, then $T$ is an eigenvector of the generator $K_{+i-i}$ with eigenvalue $\ell_{i}$. If $i<j$, i.e. we are acting with a raising operator, then the right hand side of the previous equation reads

$$
T_{+1\left(\ell_{1}\right), \ldots,+i\left(\ell_{i}\right), \ldots,+i+j\left(\ell_{j}-1\right), \ldots,+p\left(\ell_{p}\right)}=0,
$$

due to the symmetry of $T$. We can therefore conclude that the $S p(N)$ irrep carried by the space of traceless tensors with symmetry of a Young diagram $\ell$ with at most $N$ rows is a highest weight module whose highest weight coincides with $\boldsymbol{\ell}$ in the orthonormal basis.

\section{B Real forms of classical Lie groups and Lie algebras}

A real form $\mathfrak{g}_{\sigma}$ of a complex Lie algebra $\mathfrak{g}$ can be identified as a subalgebra of the realification $\mathfrak{g}^{\mathbb{R}}$ of $\mathfrak{g}$, namely

$$
\mathfrak{g}_{\sigma}:=\left\{A \in \mathfrak{g}^{\mathbb{R}} \mid \sigma(A)=-A\right\}
$$

\footnotetext{
${ }^{21}$ Notice that the Killing form $\kappa$ of $\mathfrak{s p}(N)$ is given by $\kappa\left(K_{I J}, K_{K L}\right) \propto \Omega_{I(K} \Omega_{L) J}$ so that the Cartan subalgebra generators $H_{i}=K_{+i-i}$ verify $\kappa\left(H_{i}, H_{j}\right)=\delta_{i j}$ (upon choosing the proper normalization). In other words, the weights computed later will be expressed in the orthonormal basis.
} 
where $\sigma: \mathfrak{g}^{\mathbb{R}} \rightarrow \mathfrak{g}^{\mathbb{R}}$ is an anti-involution, i.e. it satisfies $\sigma\left(\left[A_{1}, A_{2}\right]\right)=-\left[\sigma\left(A_{1}\right), \sigma\left(A_{2}\right)\right]$ and $\sigma^{2}=1$.

In the following, we summarize the list of all real forms and present the action $\sigma$ on their generators. For that, let us define first the following matrices

$$
\eta=\left(\begin{array}{cc}
I_{N_{+}} & 0 \\
0 & -I_{N_{-}}
\end{array}\right), \quad \Omega=\left(\begin{array}{cc}
0 & I_{N} \\
-I_{N} & 0
\end{array}\right),
$$

where $I_{N}$ is the $N \times N$ identity matrix.

\section{B.1 General linear Lie groups and Lie algebras}

The group of invertible $n \times n$ complex matrices, $G L(N, \mathbb{C})$, is a real Lie group of dimension $2 N^{2}$. The associated Lie algebra $\mathfrak{g l}(N, \mathbb{C})$ is the commutator Lie algebra of $N \times N$ complex matrices generated by the matrices $\mathrm{X}_{B}^{A}$ with the components $\left(\mathrm{X}^{A}{ }_{B}\right)_{C}{ }^{D}=\delta_{C}^{A} \delta_{B}^{D}$. The generators $\mathrm{X}_{B}^{A}$ satisfy the commutation relation,

$$
\left[\mathrm{X}^{A}{ }_{B}, \mathrm{X}^{C}{ }_{D}\right]=\delta_{B}^{C} \mathrm{X}^{A}{ }_{D}-\delta_{D}^{A} \mathrm{X}_{B}^{C} .
$$

For later use, it will be useful to note

$$
Y \mathrm{X}_{B}^{A}=Y_{C}^{A} \mathrm{X}_{B}^{C}, \quad \mathrm{X}_{B}^{A} Y=\mathrm{X}_{C}^{A} Y_{B}{ }^{C},
$$

where $Y_{A}^{B}$ are the components of the matrix $Y$.

All real forms of the complex Lie group/algebra $G L_{N} / \mathfrak{g l}_{N}$ are subgroups/subalgebras of $G L(N, \mathbb{C}) / \mathfrak{g l}(N, \mathbb{C})$. There are three classes of real forms:

- $G L(N, \mathbb{R})$ : the group of $N \times N$ real invertible matrices,

$$
\begin{aligned}
G L(N, \mathbb{R}) & =\left\{g \in G L(N, \mathbb{C}) \mid g^{*}=g\right\}, \\
\mathfrak{g l}(N, \mathbb{R}) & =\left\{A \in \mathfrak{g l}(N, \mathbb{C}) \mid A^{*}=A\right\},
\end{aligned}
$$

which is singled out by the anti-involution,

$$
\sigma(A)=-A^{*} .
$$

Since $\mathrm{X}_{B}^{A}$ are real, the action of $\sigma$ on the generators $\mathrm{X}^{A}{ }_{B}$ simply reads

$$
\sigma\left(\mathrm{X}_{B}^{A}\right)=-\mathrm{X}_{B}^{A} .
$$

- $U\left(N_{+}, N_{-}\right)$: the group of $\left(N_{+}+N_{-}\right) \times\left(N_{+}+N_{-}\right)$complex matrices which preserves the Hermitian form $\eta$ of signature $\left(N_{+}, N_{-}\right)$,

$$
\begin{aligned}
U\left(N_{+}, N_{-}\right) & =\left\{g \in G L\left(N_{+}+N_{-}, \mathbb{C}\right) \mid g^{\dagger} \eta g=\eta\right\}, \\
\mathfrak{u}\left(N_{+}, N_{-}\right) & =\left\{A \in \mathfrak{g l}\left(N_{+}+N_{-}, \mathbb{C}\right) \mid A^{\dagger} \eta+\eta A=0\right\},
\end{aligned}
$$

which is singled out by the anti-involution,

$$
\sigma(A)=\eta A^{\dagger} \eta
$$


Since $\left(X^{A}{ }_{B}\right)^{\dagger}=X^{B}{ }_{A}$, the action of $\sigma$ is given by

$$
\sigma\left(\mathrm{X}_{B}^{A}\right)=\eta \mathrm{X}^{B}{ }_{A} \eta=\eta_{B D} \mathrm{X}_{C}^{D} \eta^{C A}
$$

where $\eta_{A B}$ are the components of the matrix $\eta$.

- $U^{*}(2 N)$ : the group of $N \times N$ invertible matrices over the quaternions $\mathbb{H},{ }^{22}$ which can be defined as the following subgroup of $G L(2 N, \mathbb{C})$ :

$$
\begin{aligned}
U^{*}(2 N) & =\left\{g \in G L(2 N, \mathbb{C}) \mid g^{*}=\Omega g \Omega^{-1}\right\}, \\
\mathfrak{u}^{*}(2 N) & =\left\{A \in \mathfrak{g l}(2 N, \mathbb{C}) \mid A^{*}=\Omega A \Omega^{-1}\right\},
\end{aligned}
$$

and is therefore singled out by the anti-involution,

$$
\sigma(A)=-\Omega A^{*} \Omega^{-1}
$$

The action of the anti-involution $\sigma$ on $X^{A}{ }_{B}$ reads

$$
\sigma\left(\mathbf{X}_{B}^{A}\right)=-\Omega_{A C} \mathbf{X}_{D}^{C} \Omega^{D B}
$$

where $\Omega_{A B}$ and $\Omega^{A B}$ are the components of $\Omega$ and its inverse: $\Omega_{A B} \Omega^{B C}=\delta_{A}^{C}$.

\section{B.2 Orthogonal Lie groups and Lie algebras}

The complex orthogonal Lie group and Lie algebras are defined as

$$
\begin{aligned}
O(N, \mathbb{C}) & =\left\{g \in G L(N, \mathbb{C}) \mid g^{t} g=I_{N}\right\}, \\
\mathfrak{s o}(N, \mathbb{C}) & =\left\{A \in \mathfrak{g l}(N, \mathbb{C}) \mid A^{t}+A=0\right\} .
\end{aligned}
$$

All real forms of $O_{N} / \mathfrak{s o}{ }_{N}$ are subgroups/subalgebras of $O(N, \mathbb{C}) / \mathfrak{s o}(N, \mathbb{C})$. There are two classes of real forms:

- $O\left(N_{+}, N_{-}\right)$: the orthogonal group of indefinite signature, which is defined by

$$
\begin{aligned}
O\left(N_{+}, N_{-}\right) & =\left\{g \in O\left(N_{+}+N_{-}, \mathbb{C}\right) \mid g^{*}=\eta g \eta\right\}, \\
\mathfrak{s o}\left(N_{+}, N_{-}\right) & =\left\{A \in \mathfrak{s o}\left(N_{+}+N_{-}, \mathbb{C}\right) \mid A^{*}=\eta A \eta\right\},
\end{aligned}
$$

and is therefore singled out by the anti-involution,

$$
\sigma(A)=-\eta A^{*} \eta
$$

A more familiar description of $O\left(N_{+}, N_{-}\right)$is as the subgroup of $G L\left(N_{+}+N_{-}, \mathbb{R}\right)$, which preserves the (flat) metric of signature $\left(N_{+}, N_{-}\right)$. To make the link between these two definitions, we use the isomorphism,

$$
\rho_{\zeta}(A)=\zeta A \zeta
$$

\footnotetext{
${ }^{22}$ As such, it is also denoted $G L(N, \mathbb{H})$.
} 
with

$$
\zeta=\left(\begin{array}{cc}
I_{N_{+}} & 0 \\
0 & i I_{N_{-}}
\end{array}\right),
$$

which verifies $\zeta^{2}=\eta$. The Lie algebra $\rho_{\zeta}\left(\mathfrak{s o}\left(N_{+}, N_{-}\right)\right) \cong \mathfrak{s o}\left(N_{+}, N_{-}\right)$is equipped with the anti-involution $\tilde{\sigma}$ induced by $\sigma$ :

$$
\tilde{\sigma}(A):=\rho_{\zeta}\left(\sigma\left(\rho_{\zeta}^{-1}(A)\right)=-\zeta \eta\left(\zeta^{-1} A \zeta^{-1}\right)^{*} \eta \zeta=-A^{*} .\right.
$$

and is generated by the matrices $\mathrm{M}_{A B}=2 \delta_{C[A} \mathrm{X}^{C}{ }_{B]}$, whose induced Lie bracket satisfies (4.4) with $E_{A B}=\eta_{A B}$. The action of the induced anti-involution $\tilde{\sigma}$ on $\mathrm{M}_{A B}$ is simply given by

$$
\tilde{\sigma}\left(\mathrm{M}_{A B}\right)=-\mathrm{M}_{A B}
$$

- $O^{*}(2 N)$ : the quaternionic orthogonal group, defined by

$$
\begin{aligned}
& O^{*}(2 N)=\left\{g \in O(2 N, \mathbb{C}) \mid g^{*}=\Omega g \Omega^{-1}\right\}, \\
& \mathfrak{s o}^{*}(2 n)=\left\{A \in \mathfrak{s o}(2 N, \mathbb{C}) \mid A^{*}=\Omega A \Omega^{-1}\right\},
\end{aligned}
$$

and which is therefore singled out by the anti-involution

$$
\sigma(A)=-\Omega A^{*} \Omega^{-1} .
$$

It is also convenient to consider another description for $O^{*}(2 N)$, which can be obtained by the isomorphism,

$$
\rho_{U}(A)=-i U A U,
$$

where

$$
U=\frac{1}{\sqrt{2}}\left(\begin{array}{cc}
I_{N} & i I_{N} \\
i I_{N} & I_{N}
\end{array}\right),
$$

satisfies

$$
U^{2}=i J, \quad U \Omega U=\Omega,
$$

with

$$
J=\left(\begin{array}{cc}
0 & I_{N} \\
I_{N} & 0
\end{array}\right) .
$$

The components of $J$ will be denoted by $J_{A B}$. The Lie algebra $\rho_{U}\left(\mathfrak{s o}^{*}(2 N)\right) \cong$ $\mathfrak{s o}^{*}(2 N)$ is equipped with the anti-involution $\tilde{\sigma}$ induced by $\sigma$ :

$$
\tilde{\sigma}(A):=\rho_{U}\left(\sigma\left(\rho_{U}^{-1}(A)\right)\right)=i U \Omega\left(i U^{-1} A U^{-1}\right)^{*} \Omega^{-1} U=-\Omega A^{*} \Omega .
$$

The induced Lie bracket is given by $\rho_{U}([A, B])=\rho_{U}(A) J \rho_{U}(B)-\rho_{U}(B) J \rho_{U}(A)$ and hence the isomorphic Lie algebra $\rho_{U}\left(\mathfrak{s o}^{*}(2 N)\right)$ is generated by the matrices $\mathrm{M}_{A B}=$ $2 \delta_{C[A} \mathrm{X}_{B]}^{C}$ which obey the commutation relations (4.4) with $E_{A B}=J_{A B}$. The induced anti-involution of $\mathrm{M}_{A B}$ reads

$$
\tilde{\sigma}\left(\mathrm{M}_{A B}\right)=-\Omega^{A C} \mathrm{M}_{C D} \Omega^{D B},
$$

where $\Omega^{A B}$ are the components of $\Omega^{-1}$. 


\section{B.3 Symplectic Lie groups and Lie algebras}

The complex symplectic Lie group and Lie algebras is defined as the group of $2 N \times 2 N$ complex matrices preserving the canonical symplectic form $\Omega$,

$$
\begin{aligned}
S p(2 N, \mathbb{C}) & =\left\{g \in G L(2 N, \mathbb{C}) \mid g^{t} \Omega g=\Omega\right\}, \\
\mathfrak{s p}(2 N, \mathbb{C}) & =\left\{A \in \mathfrak{g l}(2 N, \mathbb{C}) \mid A^{t} \Omega+\Omega A=0\right\} .
\end{aligned}
$$

Since $\Omega A$ is a symmetric matrix, it is more convenient to consider the Lie algebra obtained by the isomorphism,

$$
\rho_{\Omega}(A)=\Omega A .
$$

The isomorphic Lie algebra $\mathfrak{s p}(2 N, \mathbb{C})$ is generated by the symmetric matrices $\mathrm{K}_{A B}=$ $2 \delta_{C(A} \mathrm{X}^{C}{ }_{B)}$ with the commutation relations,

$$
\left[\mathrm{K}_{A B}, \mathrm{~K}_{C D}\right]=2\left(\Omega_{D(A} \mathrm{K}_{B) C}+\Omega_{C(A} \mathrm{K}_{B) D}\right),
$$

where $\Omega_{A B}$ are the components of $\Omega$.

All real forms of $S p_{2 N} / \mathfrak{s p}_{2 N}$ are subgroups/subalgebras of $S p(2 N, \mathbb{C}) / \mathfrak{s p}(2 N, \mathbb{C})$. There are two types of real forms:

- $S p(2 N, \mathbb{R})$ : the group of $2 N \times 2 N$ real matrices which preserve the canonical symplectic form $\Omega$,

$$
\begin{aligned}
S p(2 N, \mathbb{R}) & =\left\{g \in S p(2 N, \mathbb{C}) \mid g^{*}=g\right\}, \\
\mathfrak{s p}(2 N, \mathbb{R}) & =\left\{A \in \mathfrak{s p}(2 N, \mathbb{C}) \mid A^{*}=A\right\},
\end{aligned}
$$

which is singled out by the anti-involution

$$
\sigma(A)=-A^{*} .
$$

This anti-involution is compatible with the isomorphism (B.30). In the case of $S p(2 N, \mathbb{R})$, it is also convenient to consider an additional isomorphism $\rho_{U}(A)=U A U$ which induces the anti-involution $\tilde{\sigma}$,

$$
\tilde{\sigma}(A)=-U\left(U^{-1} A U^{-1}\right)^{*} U=J A^{*} J,
$$

where the matrices $U$ and $J$ are defined in (B.24) and (B.26). The action of the anti-involution on $\mathrm{K}_{A B}=2 \delta_{C(A} \mathrm{X}^{C}{ }_{B)}$ reads

$$
\tilde{\sigma}\left(\mathrm{K}_{A B}\right)=J \mathrm{~K}_{A B} J=J^{A C} \mathrm{~K}_{C D} J^{D B} .
$$

Due to the second identity in (B.25), the Lie algebra $\rho_{U}(\mathfrak{s p}(2 n, \mathbb{R}))$ has the same Lie bracket as (B.31).

- $\operatorname{Sp}\left(N_{+}, N_{-}\right)$, the group of $\left(N_{+}+N_{-}\right) \times\left(N_{+}+N_{-}\right)$matrices over the quaternions that preserve the metric $\eta$ of signature $\left(N_{+}, N_{-}\right)$, which can also be defined as

$$
\begin{aligned}
& S p\left(N_{+}, N_{-}\right)=\left\{g \in S p\left(2\left(N_{+}+N_{-}\right), \mathbb{C}\right) \mid g^{\dagger} \Upsilon g=\Upsilon\right\}, \\
& \mathfrak{s p}\left(N_{+}, N_{-}\right)=\left\{A \in \mathfrak{s p}\left(2\left(N_{+}+N_{-}\right), \mathbb{C}\right) \mid A^{\dagger} \Upsilon+\Upsilon A=0\right\},
\end{aligned}
$$


where $\Upsilon$ is defined as

$$
\Upsilon=\left(\begin{array}{ll}
\eta & 0 \\
0 & \eta
\end{array}\right)
$$

The corresponding real form is therefore singled out by the anti-involution,

$$
\sigma(A)=\Upsilon A^{\dagger} \Upsilon
$$

The induced anti-involution $\tilde{\sigma}$ of $\mathrm{K}_{A B}=2 \delta_{C(A} \mathrm{X}^{C}{ }_{B)}$ reads

$$
\tilde{\sigma}\left(\mathrm{K}_{A B}\right)=\Omega \Upsilon\left(\Omega^{-1} \mathrm{~K}_{A B}\right)^{t} \Upsilon=\Psi \mathrm{K}_{A B} \Psi=\Psi^{A C} \mathrm{~K}_{C D} \Psi^{D B},
$$

where $\Psi$ is defined as

$$
\Psi=\Omega \Upsilon=\left(\begin{array}{cc}
0 & \eta \\
-\eta & 0
\end{array}\right),
$$

and $\Psi_{A B}$ and $\Psi^{A B}$ are the components of $\Psi$ and $\Psi^{-1}$.

\section{Seesaw pairs diagrams}

In section 2.3, we explained how a seasaw diagram can be obtained from a dual pair by systematically looking for the maximal compact subgroups of all groups appearing in the process. Below, we present such seasaw diagrams for all seven irreducible dual pairs listed in table 1.

\begin{tabular}{|c|c|c|c|c|}
\hline & $U(M, M)$ & $\longleftrightarrow$ & $U(N)$ & \\
\hline & $\cup$ & & $\cap$ & $c$ \\
\hline \multirow[t]{3}{*}{$U(M) \times U(M)$} & $G L(M, \mathbb{C})$ & $\longleftrightarrow$ & $G L(N, \mathbb{C})$ & $U(N) \times U(N)$ \\
\hline & $\cup$ & & $\cap$ & $?$ \\
\hline & $U(M)$ & $\longleftrightarrow$ & $U(N, N)$ & \\
\hline
\end{tabular}

- $(G L(M, \mathbb{R}), G L(N, \mathbb{R}))$

$$
\begin{aligned}
& S p(2 M, \mathbb{R}) \quad \longleftrightarrow \quad O(N) \\
& \text { C } \cup \quad \cap \quad C \\
& U(M) \quad G L(M, \mathbb{R}) \quad \longleftrightarrow \quad G L(N, \mathbb{R}) \quad U(N) \\
& O(M) \quad \longleftrightarrow \quad S p(2 N, \mathbb{R})
\end{aligned}
$$

- $(G L(M, \mathbb{C}), G L(N, \mathbb{C}))$ 
- $\left(U^{*}(2 M), U^{*}(2 N)\right)$

\begin{tabular}{|c|c|c|c|c|c|}
\hline \multirow{5}{*}{$U(2 M)$} & & $O^{*}(4 M)$ & $\longleftrightarrow$ & $S p(N)$ & \multirow[b]{3}{*}{$U(2 N)$} \\
\hline & $c$ & $\cup$ & & $\cap$ & \\
\hline & & $U^{*}(2 M)$ & $\longleftrightarrow$ & $U^{*}(2 N)$ & \\
\hline & \multirow[t]{2}{*}{$৩$} & $\cup$ & & $\cap$ & $?$ \\
\hline & & $S p(M)$ & $\longleftrightarrow$ & $O^{*}(4 N)$ & \\
\hline
\end{tabular}

- $\left(U\left(M_{+}, M_{-}\right), U\left(N_{+}, N_{-}\right)\right)$

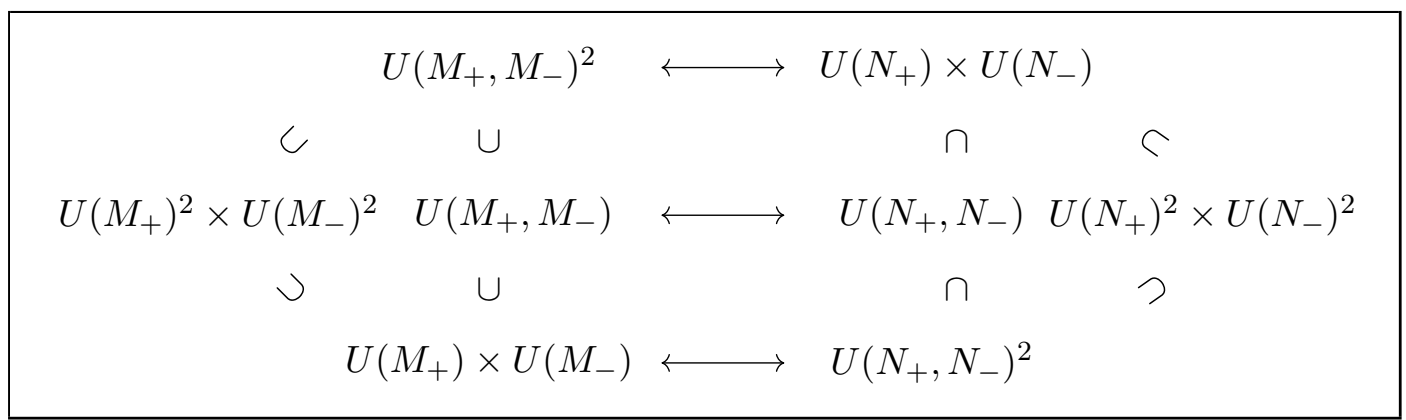

- $\left(O\left(N_{+}, N_{-}\right), S p(2 M, \mathbb{R})\right)$

\begin{tabular}{|c|c|c|c|c|}
\hline & $U\left(N_{+}, N_{-}\right)$ & $\longleftrightarrow$ & $U(M)$ & \\
\hline$c$ & $\cup$ & & $\cap$ & $\varsigma$ \\
\hline \multirow[t]{3}{*}{$U\left(N_{+}\right) \times U\left(N_{-}\right)$} & $O\left(N_{+}, N_{-}\right)$ & $\longleftrightarrow$ & $S p(2 M, \mathbb{R})$ & $U(M) \times U(M)$ \\
\hline & $\cup$ & & $\cap$ & $?$ \\
\hline & $O\left(N_{+}\right) \times O\left(N_{-}\right)$ & $\longleftrightarrow$ & $S p(2 M, \mathbb{R})^{2}$ & \\
\hline
\end{tabular}

- $(O(N, \mathbb{C}), S p(2 M, \mathbb{C}))$

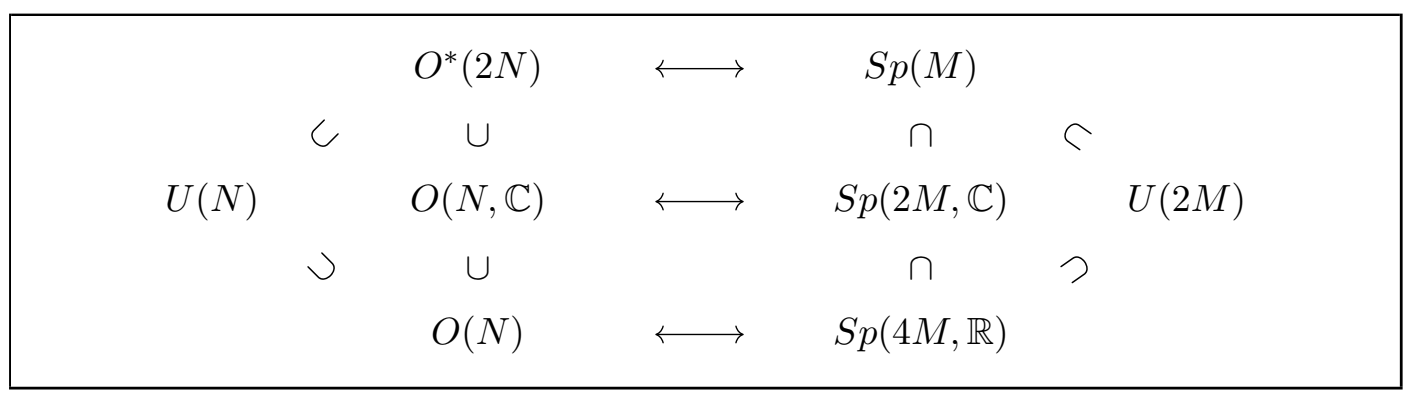


- $\left(S p\left(M_{+}, M_{-}\right), O^{*}(2 N)\right)$

\begin{tabular}{|c|c|c|c|c|}
\hline & $U\left(2 M_{+}, 2 M_{-}\right)$ & $\longleftrightarrow$ & $U(N)$ & \\
\hline$c$ & $\cup$ & & $\cap$ & $c$ \\
\hline$U\left(2 M_{+}\right) \times U\left(2 M_{-}\right)$ & $S p\left(M_{+}, M_{-}\right)$ & $\longleftrightarrow$ & $O^{*}(2 N)$ & $U(N) \times U(N)$ \\
\hline$\diamond$ & $\cup$ & & $\cap$ & $>$ \\
\hline
\end{tabular}

\section{Derivation of Casimir relations}

In the following two subsections we provide the details of the derivations leading to the duality relations, presented in the main text, between all Casimir operators of the two groups in a dual pair, in terms of their generating functions.

\section{D.1 Relating different choices of Casimir operators for the same group $G L_{N}$}

Here we derive the relation between the generating functions $r(t)$ and $\tilde{r}(t)$ of the Casimir operators of the same group $G L_{N}$ spanned by $R_{I}{ }^{J}$, with $I, J=1, \ldots, N$, as introduced in (9.1) and (9.3). In order to do that, we introduce the following notations:

$$
\begin{aligned}
\left(\boldsymbol{R}^{n}\right)_{I}{ }^{J} & =R_{I}{ }^{K_{1}} R_{K_{1}}{ }^{K_{2}} \ldots R_{K_{n-1}}{ }^{J} \\
\left(\left(\boldsymbol{R}^{t}\right)^{n}\right)_{I}{ }^{J} & =R_{K_{1}}{ }^{J} R_{K_{2}}{ }^{K_{1}} R_{K_{3}}{ }^{K_{2}} \ldots R_{I}{ }^{K_{n-1}} .
\end{aligned}
$$

These two types of operators differ only by ordering and therefore are related as

$$
\left(\left(\boldsymbol{R}^{t}\right)^{n}\right)_{I}^{J}=\sum_{m=0}^{n} a_{n m}\left(\boldsymbol{R}^{m}\right)_{I}^{J}
$$

for some $a_{n m}$ that may also depend on traces of powers of $R(9.2)$. Using $\left(\left(\boldsymbol{R}^{t}\right)^{n+1}\right)_{J}^{I}=$ $\left(\left(\boldsymbol{R}^{t}\right)^{n}\right)_{K}{ }^{I} R_{J}{ }^{K}$ and the commutation relations,

$$
\left[A_{I}^{J}, R_{K}^{L}\right]=\delta_{K}^{J} A_{I}^{L}-\delta_{I}^{L} A_{K}^{J}
$$

we derive

$$
\sum_{m=0}^{n+1} a_{n+1 m}\left(\boldsymbol{R}^{m}\right)_{J}^{I}=\sum_{m=0}^{n} a_{n m}\left[\left(\boldsymbol{R}^{m+1}\right)_{J}^{I}-N\left(\boldsymbol{R}^{m}\right)_{J}^{I}+\mathcal{C}_{m}[\boldsymbol{R}] \delta_{J}^{I}\right] .
$$

The latter equation induces recursion relations for $a_{n m}$ in the following form:

$$
\begin{aligned}
& a_{n+1 m}=a_{n, m-1}-N a_{n m} \quad(m \geq 1), \\
& a_{n+10}=\sum_{m=0}^{n} a_{n m} \mathcal{C}_{m}[\boldsymbol{R}]-N a_{n 0} .
\end{aligned}
$$


It is useful to introduce a generating function for $a_{n m}$ in the form,

$$
a(x, y)=\sum_{n, m=0}^{\infty} a_{n m} x^{n} y^{m} .
$$

In terms of the generating function, the recursion relation (D.5) takes the form:

$$
\frac{1}{x}(a(x, y)-a(x, 0))=y a(x, y)-N(a(x, y)-a(x, 0)),
$$

which is solved as

$$
a(x, y)=\frac{a(x, 0)}{1-\frac{x}{1+N x} y}=a(x, 0) \sum_{k=0}^{\infty}\left(\frac{x}{1+N x} y\right)^{k} .
$$

The second recursion relation (D.5) can be written as

$$
\frac{1}{x}(a(x, 0)-1)=\operatorname{Tr}[a(x, \boldsymbol{R})]-N a(x, 0) .
$$

Using (D.8), we get

$$
\operatorname{Tr}[a(x, \boldsymbol{R})]=a(x, 0) \sum_{k=0}^{\infty}\left(\frac{x}{1+N x}\right)^{k} \mathcal{C}_{k}[R]=a(x, 0) r\left(\frac{x}{1+N x}\right) .
$$

Now, (D.5) can be written as

$$
\frac{1}{x}(a(x, 0)-1)=a(x, 0) r\left(\frac{x}{1+N x}\right)-N a(x, 0),
$$

which can be solved by

$$
a(x, 0)=\frac{1}{1+N x-x r\left(\frac{x}{1+N x}\right)} .
$$

Then,

$$
a(x, y)=\frac{1}{\left[1+N x-x r\left(\frac{x}{1+N x}\right)\right]\left[1-\frac{x}{1+N x} y\right]}=\frac{1}{1+N x-x r\left(\frac{x}{1+N x}\right)} \sum_{k=0}^{\infty}\left(\frac{x}{1+N x} y\right)^{k},
$$

which can be used to get finally

$$
\tilde{r}(x)=\operatorname{Tr}[a(x, \boldsymbol{R})]=\frac{r\left(\frac{x}{1+N x}\right)}{1+N x-x r\left(\frac{x}{1+N x}\right)} .
$$

The inverse relation is given by

$$
r(x)=\frac{\tilde{r}\left(\frac{x}{1-N x}\right)}{1-N x+x \tilde{r}\left(\frac{x}{1-N x}\right)} .
$$

Note that these relations go to one another, if we change the sign of $N$. 


\section{D.2 Details on the Casimir relations for $\left(O_{N}, S p_{2 M}\right)$}

Here we derive the relations between Casimir operators of any order for the dual pair $\left(O_{N}, S p_{2 M}\right) \subset S p(2 N M, \mathbb{R})$. We start with repeating relevant definitions of the main text. Consider $2 M N$ operators $y_{A}^{I}$, where $A=1, \ldots, N$ and $I=1, \ldots, 2 M$, satisfying

$$
\left[y_{A}^{I}, y_{B}^{J}\right]=E_{A B} \Omega^{I J},
$$

where $E_{A B}$ is an arbitrary symmetric flat metric with eigenvalues \pm 1 , and $\Omega^{I J}$ is the $2 M$ dimensional symplectic metric, and their inverses $E^{A B}$ and $\Omega_{I J}$ are defined by

$$
E^{A B} E_{B C}=\delta_{C}^{A}, \quad \Omega^{I J} \Omega_{J K}=\delta_{K}^{I} .
$$

The generators of $O_{N}$ and $S p_{2 M}$ are realized in terms of these operators by

$$
M_{A B}=\Omega_{I J} y_{[A}^{I} y_{B]}^{J}, \quad K^{I J}=E^{A B} y_{A}^{(I} y_{B}^{J)} .
$$

It is useful to also define

$$
y_{I}^{A}:=E^{A B} \Omega_{I J} y_{B}^{J},
$$

implying the following straightforward to compute, but useful relations

$$
y_{A}^{I}=E_{A B} \Omega^{I J} y_{J}^{B}, \quad \Omega^{I J} y_{J}^{A}=E^{A B} y_{B}^{I}, \quad E_{A B} y_{I}^{B}=\Omega_{I J} y_{A}^{J},
$$

and obeying (note the sign)

$$
\left[y_{I}^{A}, y_{J}^{B}\right]=-E^{A B} \Omega_{I J} \quad\left[y_{I}^{A}, y_{B}^{J}\right]=\delta_{B}^{A} \delta_{I}^{J} .
$$

We also define

$$
N_{B}^{A}:=y_{I}^{A} y_{B}^{I}, \quad L_{J}^{I}:=y_{A}^{I} y_{J}^{A},
$$

and note that

$$
\left[N^{A}{ }_{B}, L_{J}^{I}\right]=0,
$$

with their relation to $M_{B}^{A}:=E^{A C} M_{C B}$ and $K^{I}{ }_{J}:=K^{I K} \Omega_{K J}$ being

$$
N_{B}^{A}=-M_{B}^{A}+M \delta_{B}^{A}, \quad L_{J}^{I}=-\left(K_{J}^{I}+\frac{N}{2} \delta_{J}^{I}\right) .
$$

We now introduce the generating functions of the $O_{N}$ and $S p_{2 M}$ Casimirs,

$$
\begin{aligned}
m(t) & =\sum_{n=0}^{\infty} t^{n} \mathcal{C}_{n}[\boldsymbol{M}], & k(t) & =\sum_{n=0}^{\infty} t^{n} \mathcal{C}_{n}[\boldsymbol{K}], \\
n(t) & =\sum_{n=0}^{\infty} t^{n} \operatorname{Tr}\left(\boldsymbol{N}^{n}\right), & l(t) & =\sum_{n=0}^{\infty} t^{n} \operatorname{Tr}\left(\boldsymbol{L}^{n}\right) .
\end{aligned}
$$

The matrix multiplication of $\boldsymbol{O}^{n}$ for any of the generators $\boldsymbol{M}, \boldsymbol{N}, \boldsymbol{K}, \boldsymbol{L}$ above is taken to be $\boldsymbol{O}^{n} \equiv O^{\alpha}{ }_{\gamma_{1}} O^{\gamma_{1}} \gamma_{2} \ldots O^{\gamma_{n-1}} \gamma_{n}$. By using the formal rewriting $\sum_{n=0}^{\infty} t^{n} \operatorname{Tr}\left(\boldsymbol{O}^{n}\right)=\operatorname{Tr}\left(\frac{1}{1-t \boldsymbol{O}}\right)$ and the relations in (D.23), the pairwise relations between generating functions of operators 
of the same algebra, $m$ and $n$, respectively, $k$ and $l$, given in the main text, (9.38) and (9.39), can be readily found.

To relate the generating functions $n$ and $l$, respectively, $m$ and $k$, it is useful to introduce the notation,

$$
{ }^{\alpha}[n]_{\beta}=y_{\gamma_{2}}^{\alpha} y_{\gamma_{3}}^{\gamma_{2}} \cdots y_{\beta}^{\gamma_{n}}, \quad{ }_{\alpha}[n]^{\beta}=y_{\alpha}^{\gamma_{2}} y_{\gamma_{2}}^{\gamma_{3}} \cdots y_{\gamma_{n}}^{\beta},
$$

where the greek indices are either the $O_{N}$ or $S p_{2 M}$ fundamental ones, thus giving rise to four different types of brackets, i.e.

$$
{ }^{I}[2 n]_{J}, \quad{ }^{A}[2 n]_{B}, \quad{ }^{I}[2 n+1]_{A}, \quad{ }^{A}[2 n+1]_{I},
$$

with the opposite contractions of the $y$ operators related by

$$
\begin{aligned}
{ }_{I}[2 n]^{J} & =(-1)^{n} \Omega_{I I^{\prime}}{ }^{I^{\prime}}[2 n]_{J^{\prime}} \Omega^{J^{\prime} J}, & { }_{A}[2 n]^{B} & =(-1)^{n} E_{A A^{\prime}} A^{\prime}[2 n]_{B^{\prime}} E^{B^{\prime} B}, \\
{ }_{I}[2 n+1]^{A} & =(-1)^{n} \Omega_{I J}{ }_{I J}[2 n+1]_{B} E^{B A}, & { }_{A}[2 n+1]^{I} & =(-1)^{n+1} E_{A B}{ }^{B}[2 n+1]_{J} \Omega^{J I} .
\end{aligned}
$$

Note, that ${ }_{A}[2 n]^{B}$ and ${ }_{I}[2 k]^{K}$, belonging to groups of the dual pair, commute, implying the following relation:

$$
{ }^{I}[2 n+1]_{A I}[2 l+1]^{A}={ }_{A}[2 l+1]^{I A}[2 n+1]_{I} .
$$

Thus, the four brackets in (D.27) give rise to three independent generating functions

$$
\begin{aligned}
& a(t, u)=\sum_{k, l=0}^{\infty} t^{k} u^{l I}[2 k+1]_{A I}[2 l+1]^{A}, \\
& b(t, u)=\sum_{k, l=0}^{\infty} t^{k} u^{l B}[2 k]_{A B}[2 l]^{A}, \quad d(t, u)=\sum_{k, l=0}^{\infty} t^{k} u^{l I}[2 k]_{J I}[2 l]^{J},
\end{aligned}
$$

with

$$
{ }^{\alpha}[0]_{\beta}:=\delta_{\beta}^{\alpha}, \quad{ }_{\alpha}[0]^{\beta}:=\delta_{\alpha}^{\beta},
$$

which enter in the definition of $b$ and $d$. We note that

$$
\begin{aligned}
& a(t, 0)=\frac{1}{t}(l(t)-l(0)), \quad b(t, 0)=n(t), \quad d(t, 0)=l(t), \\
& a(0, u)=\frac{1}{u}(n(-u)-n(0)), \quad b(0, u)=n(-u), \quad d(0, u)=l(-u) .
\end{aligned}
$$

Likewise, their derivatives evaluated at $t=0$ or $u=0$ are related to derivatives of $l$ and $n$. But as we will see now, it is at the point $u=-t$ that we need to understand these generating functions.

To relate the Casimirs of $O_{N}$ to those of $S p_{2 M}$, we first move the $y$ operator in the left end of $\operatorname{Tr}\left(\boldsymbol{N}^{n}\right)$ to its right end to find

$$
\operatorname{Tr}\left(\boldsymbol{N}^{n}\right)=\operatorname{Tr}\left(\boldsymbol{L}^{n}\right)+\sum_{i=0}^{n-1} \operatorname{Tr}\left(\boldsymbol{L}^{i}\right) \operatorname{Tr}\left(\boldsymbol{N}^{n-1-i}\right)-\sum_{i=0}^{n-2}(-1)^{n-2-i I}[2 i+1]_{A I}[2(n-2-i)+1]^{A}
$$


valid for $n \geq 1$, and with the last term understood as zero for $n=1$. The identity is equivalent to

$$
n(t)-N=l(t)-2 M+t l(t) n(t)-t^{2} a(t,-t),
$$

making it evident that another relation for $a(t,-t)$ in terms of $n$ and $l$ is needed. We therefore now derive relations between $a, b$, and $d$.

By moving the $y$ operator in the left end of ${ }^{I}[2 k+1]_{A}$ to its right end, we find

$$
{ }^{I}[2 k+1]_{A}={ }^{B}[2 k]_{A} y_{B}^{I}-\sum_{i=1}^{k} \operatorname{Tr}\left(\boldsymbol{N}^{i-1}\right)^{I}[2(k-i)+1]_{A}-\sum_{i=1}^{k}(-1)^{i}{ }_{B}[2 i-1]^{I B}[2(k-i)]_{A} .
$$

Considering the last term, we can pass $2 i-2$ out of the $2 i-1 y$-operators in ${ }_{B}[2 i-1]^{I}$ through ${ }^{B}[2(k-i)]_{A}$, since they commute, thus getting

$$
\begin{aligned}
{ }^{I}[2 k+1]_{A I}[2 l+1]^{A}= & { }^{B}[2 k]_{A B}[2 l+2]^{A}-\sum_{i=1}^{k} \operatorname{Tr}\left(\boldsymbol{N}^{i-1}\right){ }^{I}[2(k-i)+1]_{A I}[2 l+1]^{A} \\
& -\sum_{i=1}^{k}(-1)^{i I}[2(k-i)+1]_{A I}[2(l+i-1)+1]^{A} .
\end{aligned}
$$

Multiplying by $t^{k} u^{l}$ and summing over all $k \in \mathbb{N}$ and $l \in \mathbb{N}_{0}$, as well as making use of the following relations that will be used repeatedly,

$$
\sum_{k=1}^{\infty} \sum_{i=1}^{k} a_{k, i}=\sum_{m, n=0}^{\infty} a_{m+n+1, n+1}
$$

and

$$
\sum_{m, n=0}^{\infty} x^{m}(-y)^{n} a_{m+n}=\sum_{j=0}^{\infty} x^{j} \frac{1-\left(-\frac{y}{x}\right)^{j+1}}{1+\frac{y}{x}} a_{j}=\frac{x}{x+y} \sum_{j=1}^{\infty}\left(x^{j}+\frac{y}{x}(-y)^{j}\right) a_{j},
$$

valid for $|y / x| \neq 1$ (since use of the geometric series is needed), we find that (D.35) can be equivalently expressed as:

$$
\begin{aligned}
a(t, u)-a(0, u)= & \frac{1}{u}(b(t, u)-b(0, u)-b(t, 0)+b(0,0))-\operatorname{tn}(t) a(t, u) \\
& +\frac{t}{t+u}(u a(t, u)+t a(t,-t)), \quad \text { for }|u / t| \neq 1 .
\end{aligned}
$$

Notice that $u=-t$ is not included in the region of validity of this expression, meaning that it cannot be simply evaluated at $u=-t$. It turns out, however, that its limit at $u=-t$ is well-defined and continuous, but leads to a differential expression for $a(t,-t)$,

$$
t\left(1-t+t n(t)+t^{2} \frac{\partial}{\partial u}\right) a(t,-t)=n(t)-b(t,-t)
$$

which we will not make use of. Instead we establish more relations away from $u=-t$ to find an expression for $n$ and $l$ at $u=0$. 
By moving the $y$ operator in the left end of ${ }_{B}[2 k+2]^{A}$ to the left of ${ }^{B}[2 l]_{A}$, we find

$$
\begin{aligned}
{ }^{B}[2 k+2]_{A B}[2 l]^{A}= & { }^{I}[2 k+1]_{A I}[2 l+1]^{A}+\sum_{i=0}^{k} \operatorname{Tr}\left(\boldsymbol{L}^{i}\right)^{B}[2(k-i)]_{A B}[2 l]^{A} \\
& +\sum_{i=0}^{k-1}(-1)^{i}{ }_{I}[2 i+1]^{B I}[2(k-i)-1]_{A B}[2 l]^{A} .
\end{aligned}
$$

By splitting the middle bracket of the last term into $2(k-i-1) y$-operators, ${ }^{I}[2(k-i-1)]_{J}$, and one $y$-operator and moving them through the left and right brackets respectively, one gets

$$
\begin{aligned}
{ }^{B}[2 k+2]_{A B}[2 l]^{A}= & { }^{I}[2 k+1]_{A I}[2 l+1]^{A}+\sum_{i=0}^{k} \operatorname{Tr}\left(\boldsymbol{L}^{i}\right)^{B}[2(k-i)]_{A B}[2 l]^{A} \\
& -\sum_{i=1}^{k}(-1)^{l+k+i I}[2 l+2 i-1]_{A I}[2(k-i)+1]^{A} \\
& -\sum_{i=1}^{k} \sum_{j=0}^{l-1}(-1)^{k+i-j I}[2 i+2 j-1]_{A I}[2(k+l-i-j)-1]^{A} \\
& -\sum_{i=1}^{k} \sum_{j=0}^{l-1}(-1)^{l+i+k I}[2 i+2 j-1]_{A I}[2(k-i)+1]^{A} \operatorname{Tr}\left(\boldsymbol{N}^{l-j-1}\right) .
\end{aligned}
$$

This equation can be equivalent expressed as

$$
\begin{aligned}
\frac{1}{t}[b(t, u)-b(0, u)]= & a(t, u)+l(t) b(t, u)-\frac{t}{t+u}[u a(-u,-t)+t a(t,-t)] \\
& -\frac{t u}{(t+u)^{2}}\left[u^{2} a(-u, u)+t u(a(t, u)+a(-u,-t))+t^{2} a(t,-t)\right] \\
& +\frac{t u}{t+u}[u a(-u,-t)+t a(t,-t)] n(-u) .
\end{aligned}
$$

We continue to give relations with less details. First,

$$
\begin{aligned}
{ }^{I}[2 k+1]_{A I}[2 l+1]^{A}= & { }^{I}[2(k+1)]_{J I}[2 l]^{J}+\sum_{i=1}^{l}(-1)^{i I}[2 k+1]_{A I}[2(l-i)+1]^{A} \operatorname{Tr}\left(\boldsymbol{L}^{i-1}\right) \\
& +\sum_{i=1}^{l}(-1)^{i I}[2(k+i-1)+1]_{A I}[2(l-i)+1]^{A},
\end{aligned}
$$

is equivalent to

$$
\begin{aligned}
a(t, u)-a(t, 0)= & \frac{1}{t}(d(t, u)-l(t)-l(-u)+2 M) \\
& -u a(t, u) l(-u)-\frac{u}{t+u}(t a(t, u)+u a(-u, u)) .
\end{aligned}
$$


Next,

$$
\begin{aligned}
{ }^{I}[2 k]_{J I}[2 l+2]^{J}= & { }^{I}[2 k+1]_{A I}[2 l+1]^{A}+\sum_{i=0}^{l}{ }^{I}[2 k]_{J I}[2(l-i)]^{J}(-1)^{i} \operatorname{Tr}\left(\boldsymbol{N}^{i}\right) \\
& +\sum_{i=1}^{l}(-1)^{i+k+l I}[2(l-i)+1]_{A I}[2(k+i-1)+1]^{A} \\
& +\sum_{i=1}^{l} \sum_{j=1}^{k}(-1)^{i+j+l} \operatorname{Tr}\left(\boldsymbol{L}^{k-j}\right)^{I}[2(l-i)+1]_{A I}[2(j+i-2)+1]^{A} \\
& +\sum_{i=1}^{l} \sum_{j=1}^{k}(-1)^{l+i+j I}[2(k-j+l-i)+1]_{A I}[2(j+i-2)+1]^{A},
\end{aligned}
$$

is equivalent to

$$
\begin{aligned}
\frac{1}{u}(d(t, u)-d(t, 0))= & a(t, u)+d(t, u) n(-u)+\frac{u}{t+u}(u a(-u, u)+t a(-u,-t)) \\
& -\frac{t u}{(t+u)^{2}}\left(t^{2} a(t,-t)+t u a(-u,-t)+t u a(t, u)+u^{2} a(-u, u)\right) \\
& -\frac{t u l(t)}{t+u}(u a(-u, u)+t a(-u,-t)) .
\end{aligned}
$$

Finally, we are in a position to derive the duality relations given in (9.34) and (9.40)(9.41): eq. (D.33) provides an expression for $a(t,-t)$ in terms of $n$ and $l$ only. Using this in (D.38) and (D.42) to derive an expression for $a(t, u)$, and inserting in (D.44) to derive a relation for $d(t, u)$, we finally get from (D.46) an equation only in terms of $n$ and $l$. This equation turns out to be regular and nontrivial at $u=0$, yielding (9.34). Then from (9.38) and (9.39) one readily gets the relations (9.40) and (9.41) relating finally $m(t)$ with $k(t)$.

Open Access. This article is distributed under the terms of the Creative Commons Attribution License (CC-BY 4.0), which permits any use, distribution and reproduction in any medium, provided the original author(s) and source are credited.

\section{References}

[1] R. Howe, Remarks on classical invariant theory, Trans. Am. Math. Soc. 313 (1989) 539.

[2] R. Howe, Transcending classical invariant theory, J. Am. Math. Soc. 2 (1989) 535.

[3] P. Jordan, Der Zusammenhang der symmetrischen und linearen Gruppen und das Mehrkörperproblem, Zeitschrift für Physik 94 (1935) 531.

[4] E. Wigner, On the Consequences of the Symmetry of the Nuclear Hamiltonian on the Spectroscopy of Nuclei, Phys. Rev. 51 (1937) 106 [inSPIRE].

[5] G. Racah, Theory of complex spectra. III, Phys. Rev. 63 (1943) 367.

[6] J. French, Symplectic symmetry in the nuclear shell model, Nucl. Phys 15 (1960) 393.

[7] A. Arima and F. Iachello, The Interacting Boson Model: Cambridge Monographs on Mathematical Physics, Cambridge Univ. Press, Cambridge, UK (1987). 
[8] P.A. Dirac, Unitary Representations of the Lorentz Group, Proc. Roy. Soc. Lond. A A183 (1945) 284.

[9] H. Chandra, Infinite irreducible representations of the Lorentz group, Proc. Royal Soc. London A 189 (1947) 372.

[10] P.A.M. Dirac, Forms of Relativistic Dynamics, Rev. Mod. Phys. 21 (1949) 392 [InSPIRE].

[11] N. Mukunda, Dirac, Harish-Chandra and the unitary representations of the Lorentz group, Current Science 65 (1993) 936.

[12] E. Majorana, Relativistic theory of particles with arbitrary intrinsic angular momentum, Nuovo Cim. 9 (1932) 335 [inSPIRE].

[13] V. Bargmann, Irreducible unitary representations of the Lorentz group, Annals Math. 48 (1947) 568 [INSPIRE].

[14] I.M. Gel'fand and M.A. Naimark, Unitary representations of the Lorentz group, Izvestiya Rossiiskoi Akademii Nauk. Seriya Matematicheskaya 11 (1947) 411.

[15] P.A.M. Dirac, A Remarkable representation of the $3+2$ de Sitter group, J. Math. Phys. 4 (1963) 901 [INSPIRE].

[16] B.N. Kursunoglu, Modern quantum theory, Freeman (1962).

[17] G. Mack and I. Todorov, Irreducibility of the ladder representations of u(2,2) when restricted to the Poincaré subgroup, J. Math. Phys. 10 (1969) 2078 [InSPIRE].

[18] W. Ruehl, Field representations of the conformal group with continuous mass spectrum, Commun. Math. Phys. 30 (1973) 287 [INSPIRE].

[19] R. Penrose, Twistor algebra, J. Math. Phys. 8 (1967) 345 [InSPIRE].

[20] R. Penrose, Twistor quantization and curved space-time, Int. J. Theor. Phys. 1 (1968) 61 [INSPIRE].

[21] R. Penrose and M.A.H. MacCallum, Twistor theory: An Approach to the quantization of fields and space-time, Phys. Rept. 6 (1972) 241 [inSPIRE].

[22] T.N. Bailey, T.N. Bailey, R. Baston and N. Hitchin, Twistors in mathematics and physics, Cambridge University Press (1990).

[23] I.E. Segal, Foundations of the theory of dynamical systems of infinitely many degrees of freedom, II, Mat. Fys. Medd. Dan. Vid. Selsk. 13 (1961) 1.

[24] D. Shale, Linear symmetries of free boson fields, Trans. Am. Math. Soc. 103 (1962) 149.

[25] A. Weil, Sur certains groupes d'opérateurs unitaires, Acta Math. 111 (1964) 143.

[26] D. Prasad, Weil representation, Howe duality, and the theta correspondence, Theta functions: from the classical to the modern 1 (1993) 105.

[27] S. Kudla, Notes on the local theta correspondence, (1996), unpublished notes available at http://www.math.toronto.edu/ skudla/castle.pdf.

[28] J. Adams, The theta correspondence over R, Harmonic Analysis, Group Representations, Automorphic Forms, and Invariant Theory (2007), pages 1-39, https://pdfs.semanticscholar.org/748b/8e1dd0ce860b37e3c11c7737a409afee09e1.pdf.

[29] B. Pioline and A. Waldron, Automorphic forms: A Physicist's survey, Les Houches School of Physics: Frontiers in Number Theory, Physics and Geometry (2007) 277 [hep-th/0312068] [INSPIRE].

[30] P. Fleig, H.P.A. Gustafsson, A. Kleinschmidt and D. Persson, Eisenstein series and automorphic representations, Cambridge University Press (2018). 
[31] A. Barut and L. Girardello, New "coherent" states associated with non-compact groups, Commun. Math. Phys. 21 (1971) 41.

[32] A.M. Perelomov, Coherent states for arbitrary Lie group, Commun. Math. Phys. 26 (1972) 222.

[33] R. Gilmore, Geometry of symmetrized states, Ann. Phys. 74 (1972) 391.

[34] M. Günaydin and N. Marcus, The Spectrum of the $S^{5}$ Compactification of the Chiral $N=2, D=10$ Supergravity and the Unitary Supermultiplets of U(2, 2/4), Class. Quant. Grav. 2 (1985) L11 [INSPIRE].

[35] M. Günaydin, D. Minic and M. Zagermann, $4 D$ doubleton conformal theories, CPT and IIB string on $A d S_{5} \times S^{5}$, Nucl. Phys. B 534 (1998) 96 [Erratum ibid. 538 (1999) 531] [hep-th/9806042] [inSPIRE].

[36] M. Günaydin and N.P. Warner, Unitary Supermultiplets of Osp $(8 / 4, r)$ and the Spectrum of the $S_{7}$ Compactification of Eleven-dimensional Supergravity, Nucl. Phys. B 272 (1986) 99 [INSPIRE].

[37] M. Günaydin and R.J. Scalise, Unitary Lowest Weight Representations of the Noncompact Supergroup Osp $\left(2 m^{*} / 2 n\right)$, J. Math. Phys. 32 (1991) 599 [inSPIRE].

[38] M. Günaydin, P. van Nieuwenhuizen and N.P. Warner, General Construction of the Unitary Representations of Anti-de Sitter Superalgebras and the Spectrum of the $S^{4}$ Compactification of Eleven-dimensional Supergravity, Nucl. Phys. B 255 (1985) 63 [INSPIRE].

[39] M. Günaydin and S. Takemae, Unitary supermultiplets of $O S p\left(8^{*}-4\right)$ and the $A d S_{7} / C F T_{6}$ duality, Nucl. Phys. B 578 (2000) 405 [Erratum ibid. 697 (2004) 399] [hep-th/9910110] [INSPIRE].

[40] M. Günaydin and C. Saclioglu, Oscillator Like Unitary Representations of Noncompact Groups With a Jordan Structure and the Noncompact Groups of Supergravity, Commun. Math. Phys. 87 (1982) 159 [INSPIRE].

[41] M. Günaydin and C. Saclioglu, Bosonic Construction of the Lie Algebras of Some Noncompact Groups Appearing in Supergravity Theories and Their Oscillator Like Unitary Representations, Phys. Lett. B 108 (1982) 180 [InSPIRE].

[42] I. Bars and M. Günaydin, Unitary Representations of Noncompact Supergroups, Commun. Math. Phys. 91 (1983) 31 [inSPIRE].

[43] E.S. Fradkin and M.A. Vasiliev, Superalgebra of Higher Spins and Auxiliary Fields, Int. J. Mod. Phys. A 3 (1988) 2983 [inSPIRE].

[44] S.E. Konstein and M.A. Vasiliev, Extended Higher Spin Superalgebras and Their Massless Representations, Nucl. Phys. B 331 (1990) 475 [INSPIRE].

[45] E.S. Fradkin and V. Linetsky, Conformal superalgebras of higher spins, Annals Phys. 198 (1990) 252 [INSPIRE].

[46] E.S. Fradkin and V. Linetsky, Conformal superalgebras of higher spins, Mod. Phys. Lett. A 04 (1989) 2363 [INSPIRE].

[47] M.A. Vasiliev, Consistent equation for interacting gauge fields of all spins in (3+1)-dimensions, Phys. Lett. B 243 (1990) 378 [INSPIRE].

[48] M.A. Vasiliev, Properties of equations of motion of interacting gauge fields of all spins in (3+1)-dimensions, Class. Quant. Grav. 8 (1991) 1387 [INSPIRE].

[49] M.A. Vasiliev, More on equations of motion for interacting massless fields of all spins in (3+1)-dimensions, Phys. Lett. B 285 (1992) 225 [INSPIRE]. 
[50] E. Sezgin and P. Sundell, Doubletons and 5-D higher spin gauge theory, JHEP 09 (2001) 036 [hep-th/0105001] [INSPIRE].

[51] E. Sezgin and P. Sundell, $7-D$ bosonic higher spin theory: Symmetry algebra and linearized constraints, Nucl. Phys. B 634 (2002) 120 [hep-th/0112100] [INSPIRE].

[52] M.A. Vasiliev, Nonlinear equations for symmetric massless higher spin fields in (A)dS(d), Phys. Lett. B 567 (2003) 139 [hep-th/0304049] [INSPIRE].

[53] M.A. Vasiliev, Higher spin superalgebras in any dimension and their representations, JHEP 12 (2004) 046 [hep-th/0404124] [INSPIRE].

[54] K.B. Alkalaev, M. Grigoriev and I. Tipunin, Massless Poincaré modules and gauge invariant equations, Nucl. Phys. B $\mathbf{8 2 3}$ (2009) 509 [arXiv:0811.3999] [inSPIRE].

[55] N. Boulanger, C. Iazeolla and P. Sundell, Unfolding Mixed-Symmetry Fields in AdS and the BMV Conjecture. II. Oscillator Realization, JHEP 07 (2009) 014 [arXiv:0812.4438] [INSPIRE].

[56] K.B. Alkalaev and M. Grigoriev, Unified BRST description of AdS gauge fields, Nucl. Phys. B 835 (2010) 197 [arXiv:0910.2690] [INSPIRE].

[57] X. Bekaert and M. Grigoriev, Manifestly conformal descriptions and higher symmetries of bosonic singletons, SIGMA 6 (2010) 038 [arXiv:0907.3195] [INSPIRE].

[58] K. Alkalaev and M. Grigoriev, Unified BRST approach to (partially) massless and massive AdS fields of arbitrary symmetry type, Nucl. Phys. B 853 (2011) 663 [arXiv:1105.6111] [INSPIRE].

[59] X. Bekaert and M. Grigoriev, Higher order singletons, partially massless fields and their boundary values in the ambient approach, Nucl. Phys. B 876 (2013) 667 [arXiv:1305.0162] [INSPIRE].

[60] E. Joung and K. Mkrtchyan, Notes on higher-spin algebras: minimal representations and structure constants, JHEP 05 (2014) 103 [arXiv: 1401.7977] [INSPIRE].

[61] K.B. Alkalaev, M. Grigoriev and E.D. Skvortsov, Uniformizing higher-spin equations, J. Phys. A 48 (2015) 015401 [arXiv: 1409.6507] [InSPIRE].

[62] K.B. Alkalaev, Global and local properties of AdS 2 higher spin gravity, JHEP 10 (2014) 122 [arXiv:1404.5330] [INSPIRE].

[63] A. Chekmenev and M. Grigoriev, Boundary values of mixed-symmetry massless fields in AdS space, Nucl. Phys. B 913 (2016) 769 [arXiv:1512.06443] [INSPIRE].

[64] E. Joung and K. Mkrtchyan, Partially-massless higher-spin algebras and their finite-dimensional truncations, JHEP 01 (2016) 003 [arXiv: 1508.07332] [INSPIRE].

[65] K.B. Alkalaev and M.A. Grigoriev, Continuous spin fields of mixed-symmetry type, JHEP 03 (2018) 030 [arXiv: 1712.02317] [INSPIRE].

[66] M. Grigoriev and E.D. Skvortsov, Type-B Formal Higher Spin Gravity, JHEP 05 (2018) 138 [arXiv: 1804.03196] [INSPIRE].

[67] M.A. Vasiliev, Multiparticle extension of the higher-spin algebra, Class. Quant. Grav. 30 (2013) 104006 [arXiv:1212.6071] [INSPIRE].

[68] M.A. Vasiliev, From Coxeter Higher-Spin Theories to Strings and Tensor Models, JHEP 08 (2018) 051 [arXiv: 1804.06520] [inSPIRE].

[69] K. Alkalaev and X. Bekaert, Towards higher-spin AdS $S_{2} / C F T_{1}$ holography, JHEP 04 (2020) 206 [arXiv: 1911.13212] [INSPIRE].

[70] D.J. Rowe, M.J. Carvalho and J. Repka, Dual pairing of symmetry groups and dynamical groups in physics, Rev. Mod. Phys. 84 (2012) 711 [arXiv:1207.0148] [INSPIRE]. 
[71] R. Goodman and N.R. Wallach, Symmetry, representations, and invariants, Graduate Texts in Mathematics 255, Springer (2009).

[72] S.-J. Cheng and W. Wang, Dualities and representations of Lie superalgebras, American Mathematical Soc. (2012).

[73] V. Bargmann, On a Hilbert space of analytic functions and an associated integral transform part I, Commun. Pure Appl. Math. 14 (1961) 187.

[74] I.E. Segal and G.W. Mackey, Mathematical problems of relativistic physics, vol. 2. American Mathematical Soc. (1963).

[75] S.-J. Cheng and W. Wang, Dualities for Lie superalgebras, arXiv:1001.0074.

[76] I. Todorov, Minimal representations and reductive dual pairs in conformal field theory, AIP Conf. Proc. 1243 (2010) 13 [arXiv: 1006.1981] [INSPIRE].

[77] H. Rubenthaler, Les paires duales dans les algèbres de Lie réductives, Astérisque 219, Société mathématique de France (1994).

[78] K. Magaard and G. Savin, Exceptional $\Theta$-Correspondences I, Compositio Mathematica 107 (1997) 89.

[79] M. Schmidt, Classification and partial ordering of reductive Howe dual pairs of classical Lie groups, J. Geom. Phy. 29 (1999) 283.

[80] S.S. Kudla, On the local theta-correspondence, Invent. math. 83 (1986) 229.

[81] M. Kashiwara and M. Vergne, On the Segal-Shale-Weil representations and harmonic polynomials, Invent. math. 44 (1978) 1.

[82] F.A. Dolan, Character formulae and partition functions in higher dimensional conformal field theory, J. Math. Phys. 47 (2006) 062303 [hep-th/0508031] [INSPIRE].

[83] B. Henning and T. Melia, Conformal-helicity duality $\&$ the Hilbert space of free CFTs, arXiv: 1902.06747 [INSPIRE].

[84] B. Henning and T. Melia, Constructing effective field theories via their harmonics, Phys. Rev. D 100 (2019) 016015 [arXiv:1902.06754] [inSPIRE].

[85] M. Flato and C. Fronsdal, One Massless Particle Equals Two Dirac Singletons: Elementary Particles in a Curved Space. 6., Lett. Math. Phys. 2 (1978) 421 [INSPIRE].

[86] M. Laoues, Massless particles in arbitrary dimensions, Rev. Math. Phys. 10 (1998) 1079 [hep-th/9806101] [INSPIRE].

[87] J.-B. Bae, E. Joung and S. Lal, One-loop test of free $\mathrm{SU}(N)$ adjoint model holography, JHEP 04 (2016) 061 [arXiv: 1603.05387] [INSPIRE].

[88] K. Govil and M. Günaydin, Deformed Twistors and Higher Spin Conformal (Super-)Algebras in Four Dimensions, JHEP 03 (2015) 026 [arXiv: 1312.2907] [INSPIRE].

[89] S. Fernando, M. Günaydin and S. Takemae, Supercoherent states of $\operatorname{OSp}\left(8^{*}-2 N\right)$, conformal superfields and the $A d S_{7} / C F T_{6}$ duality, Nucl. Phys. B $\mathbf{6 2 8}(2002) 79$ [hep-th/0106161] [INSPIRE].

[90] T. Garidi, J.P. Gazeau and M.V. Takook, 'Massive' spin two field in de Sitter space, J. Math. Phys. 44 (2003) 3838 [hep-th/0302022] [INSPIRE].

[91] S. Behroozi, S. Rouhani, M.V. Takook and M.R. Tanhayi, Conformally invariant wave equations and massless fields in de Sitter spacetime, Phys. Rev. D 74 (2006) 124014 [gr-qc/0512105] [INSPIRE].

[92] J.-P. Gazeau, P. Siegl and A. Youssef, Krein Spaces in de Sitter Quantum Theories, SIGMA 6 (2010) 011 [arXiv:1001.4810] [InSPIRE]. 
[93] J. Dixmier, Représentations intégrables du groupe de De Sitter, Bulletin de la Société Mathématique de France 89 (1961) 9.

[94] V.K. Dobrev, G. Mack, V.B. Petkova, S.G. Petrova and I.T. Todorov, Harmonic Analysis on the n-Dimensional Lorentz Group and Its Application to Conformal Quantum Field Theory, Springer-Verlag (1977).

[95] B. Nagel, Spectra and Generalized Eigenfunctions of the One- and Two-Mode Squeezing Operators in Quantum Optics, Math. Phys. Stud. 18 (1995) 211 [quant-ph/9711018] [INSPIRE].

[96] N. Vilenkin and A. Klimyk, Representation of Lie groups and special functions. Volume 3: Classical and quantum groups and special functions, in Mathematics and Its Applications (Soviet Series) book series 75 Springer Science \& Business Media (2013).

[97] T. Kobayashi, B. Orsted and M. Pevzner, Geometric analysis on small unitary representations of GL (N, R), J. Funct. Anal. 260 (2011) 1682 [arXiv:1002.3006].

[98] R. Howe and S.T. Lee, Degenerate principal series representations of $G L_{n}(\mathbb{C})$ and $G L_{n}(\mathbb{R})$, J. Funct. Anal. 166 (1999) 244.

[99] S.T. Lee, Covariants of $S p_{n}(\mathbb{C})$ and degenerate principal series of $G L_{n}(\mathbb{H})$, J. Funct, Anal. 253 (2007) 18.

[100] X. Bekaert, Singletons and their maximal symmetry algebras, in 6th Summer School in Modern Mathematical Physics 11 (2011) 71, arXiv:1111.4554 [INSPIRE].

[101] J.B. Ehrman, On the unitary irreducible representations of the universal covering group of the $3+2$ deSitter group, Mathe. Proc. Cambridge Philosophical Society 53 (1957) 290.

[102] E. Angelopoulos and M. Laoues, Singletons on AdS(n), Math. Phys. Stud. 21-22 (2000) 3.

[103] M.A. Vasiliev, On Conformal, $\mathrm{SL}(4, \mathbb{R})$ and $S p(8, R)$ Symmetries of $4 d$ Massless Fields, Nucl. Phys. B 793 (2008) 469 [arXiv:0707.1085] [InSPIRE].

[104] W.H. Klink and T. Ton-That, On resolving the multiplicity of arbitrary tensor products of the U(N) groups, J. Phys. A 21 (1988) 3877.

[105] E.Y. Leung, On resolving the multiplicity of tensor products of irreducible representations of symplectic groups, J. Phys. A 26 (1993) 5851.

[106] E.Y. Leung and T. Ton-That, Invariant Theory of the Dual Pairs $\left(\mathrm{SO}^{*}(2 n), \operatorname{Sp}(2 k, \mathbb{C})\right)$ and $(\mathrm{Sp}(2 n, \mathbb{R}), \mathrm{O}(N))$, Proc. Am. Math Soc. 120 (1994) 53.

[107] M. Itoh, Correspondences of the Gelfand invariants in reductive dual pairs, J. Australian Math. Soc. 75 (2003) 263.

[108] P. Vogel, The Universal Lie algebra, (1999), unpublished notes available at https://webusers.imj-prg.fr/ pierre.vogel/grenoble-99b.pdf.

[109] R.L. Mkrtchyan, A.N. Sergeev and A.P. Veselov, Casimir eigenvalues for universal Lie algebra, J. Math. Phys. 53 (2012) 102106 [arXiv:1105.0115].

[110] M.Y. Avetisyan and R.L. Mkrtchyan, On universal quantum dimensions of certain two-parameter series of representations, arXiv:1909.02076 [INSPIRE].

[111] R.L. Mkrtchian, The Equivalence of $S p(2 N)$ and $S O(-2 N)$ Gauge Theories, Phys. Lett. B 105 (1981) 174 [INSPIRE].

[112] R.L. Mkrtchyan and A.P. Veselov, On duality and negative dimensions in the theory of Lie groups and symmetric spaces, J. Math. Phys. 52 (2011) 083514 [arXiv:1011.0151] [INSPIRE].

[113] M. Hamermesh, Group Theory and Its Application to Physical Problems, Addison Wesley Series in Physics, Dover Publications (1989). 